\title{
As 2-álgebras de Lie simples de posto toral 3.
}

\author{
Carlos Rafael Payares Guevara
}

TESE APRESENTADA

$\mathrm{AO}$

Instituto de MatemáticA E EstatísticA

DA

Universidade de SÃo PAUlo

PARA

OBTENÇÃO DO TÍTULO

DE

Doutor EM CiÊnCIAS

Programa: Matemáticas

Orientador: Prof. Dr. Alexandre Grichkov

Durante o desenvolvimento deste trabalho o autor recebeu auxilio financeiro da CAPES

Sao Paulo, Dezembro de 2016 


\section{As 2-álgebras de Lie simples de posto toral 3.}

Esta é a versão original da tese elaborada pelo candidato (Carlos Rafael Payares Guevara), tal como submetida a Comissão Julgadora. 


\section{As 2-álgebras de Lie simples de posto toral 3.}

Esta versão da tese contém as correções e alterações sugeridas pela Comissão Julgadora durante a defesa da versão original do trabalho, realizada em 05/12/2016. Uma cópia da versão original está disponível no Instituto de Matemática e Estatística da Universidade de São Paulo.

Comissão Julgadora:

- Prof. Dr. Henrique Guzzo Junior (Presidente) - IME-USP

- Prof. Dr. Juan Carlos Gutierrez Fernandez - IME-USP

- Prof. Dr. Wilian Francisco de Araujo - UTFPr

- Prof. Dr. Artem Lopatin - UNICAMP

- Prof. Dr. IIya Gorshkov - UFABC 



\section{Agradecimentos}

Agradeço, primeiramente à Deus, por sua infinita misericórdia e fidelidade.

Aos meus pais, Armando Payares H., e Marlene Guevara H., razão maior da minha existência.

À minha esposa e companheira incondicional Mariela Perez A., pela paciência, amor e compreensão nas minhas ausências.

Agradeço a CAPES pelo importante apoio financeiro.

À minha família, pelo apoio, incentivo, compreensão e paciência.

Quero agradecer ao Prof. Dr. Alexandre Grichkov, pela excelente orientação.

Aos membros da banca pelas correções, sugestões e orientações, para a versão final da tese.

Sou grato aos meus amigos de doutorado, pela força e amizade.

\section{Muito obrigado Brasil...!}





\section{Resumo}

PAYARES GUEVARA, C. R. As 2-álgebras de Lie simples de posto toral 3.

2016. 120 f. Tese (Doutorado) - Instituto de Matemática e Estatística, Universidade de Sao Paulo, Sao Paulo, 2016.

Neste trabalho estudamos as 2-álgebras de Lie simples, de dimensão finita e de posto toral 3, sobre um corpo algebricamente fechado de característica 2. Nós conjecturamos que a única 2-álgebra de Lie simples de este tipo é $W(1, \underline{3})$. Assim, nosso principal objetivo é verificar a veracidade desta conjectura para estas álgebras de pequenas dimensões.

Como resultados, provamos que esta conjectura é certa para todas estes álgebras de dimensão menor ou igual a 16, e também em algumos casos especias quando a dimensão é 17 .

Palavras-chave: 2-álgebras de Lie , Posto toral, Simples. 



\begin{abstract}
PAYARES GUEVARA, C. R. Simple Lie 2-algebras of toral rank 3.

2016. 120 f. Tese (Doutorado) - Instituto de Matemática e Estatística, Universidade de Sao Paulo, Sao Paulo, 2016.

In this work we study the simple Lie 2-algebras of finite dimension, and toral rank 3 over an algebraically closed field characteristic 2 . We surmise that the only simple Lie 2 -algebra of this type is $W(1, \underline{3})$. So, our main objective is to study the truthful of this conjecture for these algebras of small dimensions. As a result, we prove that this conjecture is true for all these algebras less than or equal to 16 dimension, and also in some special cases when the dimension is 17 .
\end{abstract}

Keywords: Lie 2-algebras, Toral rank, Simple. 



\section{Conteúdo}

$\begin{array}{ll}\text { Introdução } & 5\end{array}$

1 Preliminares e Notações $\quad 9$

1.1 Categoria das 2-álgebras de Lie . . . . . . . . . . . . . . . . . 9

1.2 Subalgebra toral e posto toral . . . . . . . . . . . . . . . . . 11

1.3 Decomposição de Cartan . . . . . . . . . . . . . . . . . . . . . . . 12

1.4 Uma 2-álgebra de Lie simples de dimensão 34 de posto toral 4 . . . . . 13

1.5 Extensões de $(L,[2])$-módulos e Lema de Schur. . . . . . . . . . . . . . 19

1.6 Potência exterior de módulos . . . . . . . . . . . . . . . . . . 19

$2 \quad$ As 2-álgebras de Lie simples de $\operatorname{dim}_{k} \leq 16$ de posto toral $3 \quad 21$

2.1 As 2-álgebras de Lie simples de $\operatorname{dim}_{k} \leq 6 \ldots \ldots \ldots$. . . . . . . . . 21

2.2 Decomposição de Cartan de uma 2-álgebra de Lie de posto toral 3. . . . 21

2.3 Análise de $L=H \oplus \sum_{\xi \in \Delta_{i}} \oplus L_{\xi}$, com $\operatorname{card}\left(\Delta_{i}\right)<7 \ldots \ldots \ldots . . \ldots 25$

2.4 Análise de $L=H \oplus \sum_{\xi \in \Delta} \oplus L_{\xi}$, com $\Delta=T^{*} \backslash\{0\} . \ldots \ldots \ldots$

3 Extensões de $\mathfrak{s l}_{3}(k)$-2-módulos $\quad 39$

$3.1 \mathrm{Os}_{\mathfrak{s}}(k)$-2-módulos irredutíveis de $\operatorname{dim}_{k} \leq 8 \ldots \ldots \ldots$. . . . . . . . . . 39

3.1 .1 2-representação irredutível de peso $(0,0) \ldots \ldots . \ldots . \ldots 39$

3.1 .2 2-representação irredutível de peso $(1,0) \ldots \ldots$. . . . . . . . 40

3.1.3 2-representação irredutível de peso $(0,1) \ldots \ldots$. . . . . . . . 40

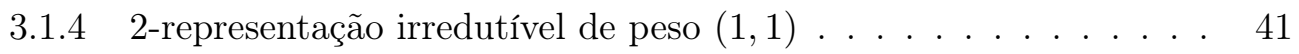

$3.2 \mathfrak{s l}_{3}(k)$-2-módulos de $\operatorname{dim}_{k}<8 \ldots \ldots \ldots \ldots$. . . . . . . . . . . 42

$3.2 .1 \quad \mathfrak{s l}_{3}(k)$-2-módulos de $\operatorname{dim}_{k}=2 \ldots \ldots \ldots \ldots \ldots$. . . . . . . . 42

$3.2 .2 \quad \mathfrak{s l}_{3}(k)$-2-módulos de $\operatorname{dim}_{k}=4 \ldots \ldots \ldots \ldots$

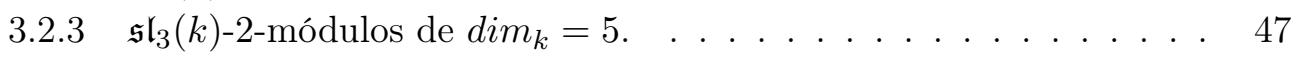

$3.2 .4 \mathfrak{s l}_{3}(k)$-2-módulos de $\operatorname{dim}_{k}=6 \ldots \ldots \ldots \ldots$. . . . . . . . . 48

$3.2 .5 \quad \mathfrak{s l}_{3}(k)$-2-módulos de $\operatorname{dim}_{k}=7 \ldots \ldots \ldots \ldots \ldots \ldots$

$3.3 \mathfrak{s l}_{3}(k)$-2-módulos de $\operatorname{dim}_{k}=8 \ldots \ldots \ldots \ldots \ldots \ldots \ldots$

3.3.1 Tabelas de alguns $\mathfrak{s l}_{3}(k)$-2-módulos de $\operatorname{dim}_{k}=8 \ldots \ldots \ldots 1$ 
$4 \quad$ As 2-álgebras de Lie simples de $\operatorname{dim}_{k}=17$ de posto toral $3 \quad 103$

$4.1 \quad L=T \oplus \sum_{\xi \in G} \oplus L_{\xi}$, onde $\operatorname{dim}_{k}\left(L_{\xi}\right)=2, \forall \xi \in G . \ldots \ldots \ldots$

4.1.1 $(L,[2])$ contém uma 2-subálgebra isomorfa a $\left(\mathfrak{s l}_{3}(k), 2\right) \ldots \ldots$

4.1.2 ( $L,[2])$ contém 2-subálgebras de Lie não simples e não solúveis. . 120 


\section{Lista de Símbolos}

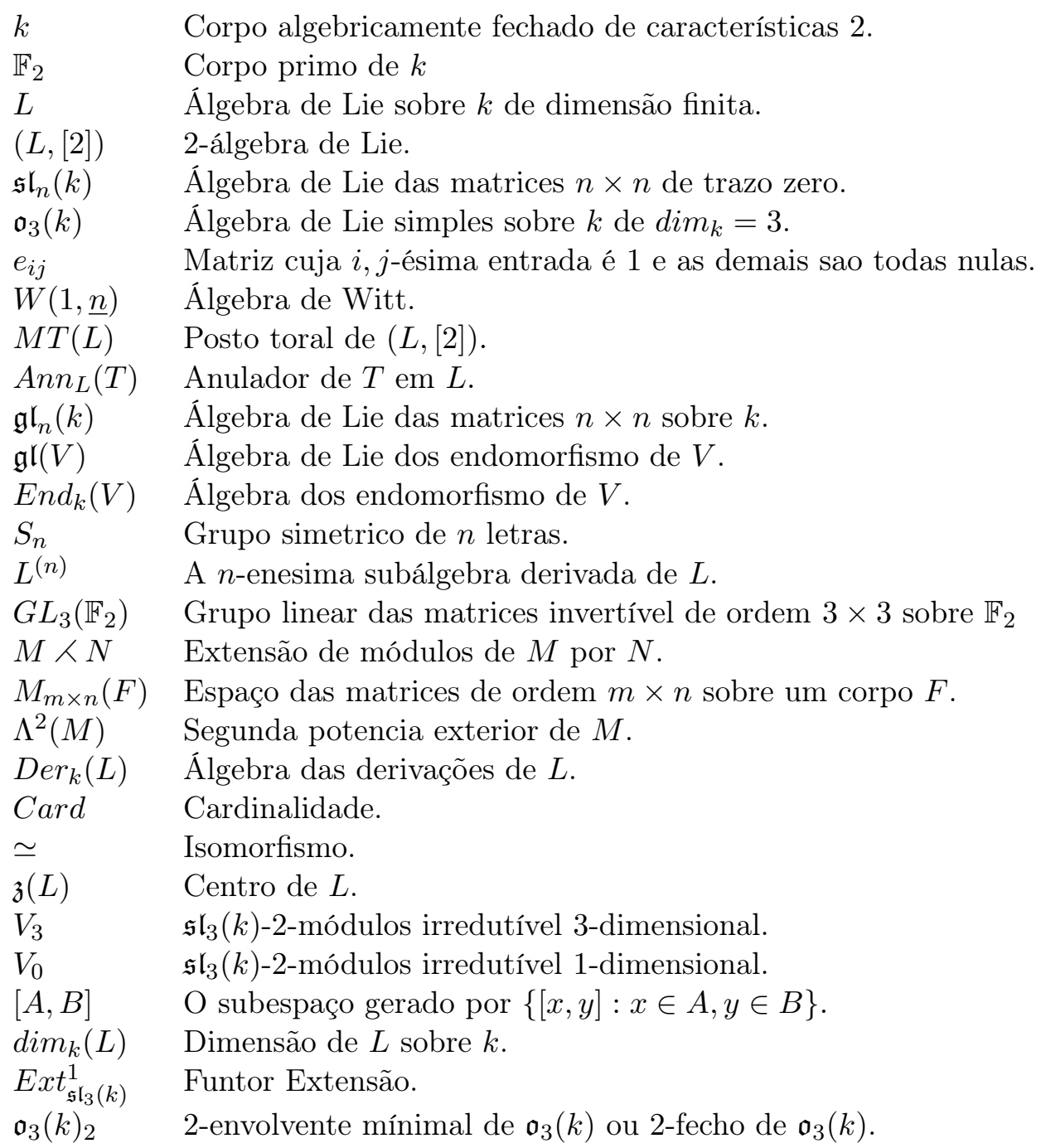





\section{Introdução}

A classificação das álgebras de Lie simples de dimensão finita depende da característica do seu corpo base. Como a característica de qualquer corpo é um número primo $p$ ou é igual a 0 , vamos resumir os resultados encontrados até agora sobre o problema de classificação das álgebras de Lie simples de dimensão finita sobre um corpo algebricamente fechado de característica zero ou prima. As álgebras de Lie simples de dimensão finita sobre um corpo algebricamente fechado de característica zero foram classificadas no início do século XIX por Killing e Cartan da seguinte forma (ver [Ja]):

Teorema 0.1 (W. Killing-E. Cartan, 1887-1894.). Toda álgebra de Lie simples de dimensão finita sobre um corpo algebricamente fechado de característica 0 é isomorfa a uma das álgebras de Lie Clássicas $A_{n}(n \geq 1), B_{n}(n \geq 2), C_{n}(n \geq 3)$, e $D_{n}(n \geq 4)$ ou as álgebras de Lie Excepcionais $G_{2}, F_{4}, E_{6}, E_{7}, E_{8}$.

O problema de classificação das álgebras de Lie simples de dimensão finita sobre um corpo algebricamente fechado de característica prima $p>0$, començuo com la seguinte conjetura de Kostrikin-Shafarevich.

Conjectura (Kostrikin - Shafarevich, 1966). Toda p-álgebra (ver [Ja] pág. 187188, definição 4.) de Lie simples de dimensão finita sobre um corpo algebricamente fechado de característica $p>5$ é do

- tipo Clássica (via álgebras de Chevalley), ou

- graduada do tipo Cartan: $W(n ; \underline{1}), n \geq 1, S(n ; \underline{1})^{(1)}, n \geq 3, H(2 r ; \underline{1})^{(2)}, r \geq 1$, $K(2 r+1 ; \underline{1})^{(1)}, r \geq 1$.

Mais tarde, Victor Kac, generaliza a conjectura de Kostrikin-Shafarevich da seguinte forma:

Conjectura (V. Kac 1971). Toda álgebra de Lie simples de dimensão finita sobre um corpo algebricamente fechado de característica $p>3$ é do tipo Clássico ou filtrada do tipo Cartan.

Como resultados parciais para estas conjecturas temos:

$\triangleright$ A conjectura de Kostrikin-Shafarevich é verdadeira para $p>7$ (R. Block e R. Wilson, 1988).

$\triangleright$ A conjetura de V. Kac é verdadeira para $p>7$ (H. Strade, 1998). 
$\triangleright$ Álgebras de Melikian, só em $p=5$. (G. Melikian, 1980).

$\triangleright$ A conjectura de V. Kac foi resolvida por H. Strade e A. Premet, no início dos anos 2.000 .

Um resumo desses resultados pode ser enunciado como o teorema de classificação de Block-Wilson-Strade-Premet, cuja demonstração pode ser encontrada em [St1], [St2], [St3] :

Teorema 0.2 (Block - Wilson - Strade - Premet, 1988-2008). Toda álgebra de Lie simples de dimensão finita sobre um corpo algebricamente fechado de característica $p>3$ é tipo Clássica (via álgebras de Chevalley), ou filtrada de tipo Cartan, ou uma álgebra de Melikian (só se $p=5$ ).

Portanto temos que

Teorema 0.3 (Classificação das $p$-álgebras de Lie simples, $p>3$ ). Toda p-álgebra de Lie simples de dimensão finita sobre um corpo algebricamente fechado de característica $p>3$ é :

- tipo Clássica: $A_{n}, \mathfrak{p s l}_{2 n}(k), n \geq 1, B_{n}, n \geq 2, C_{n}, n \geq 3, D_{n}, n \geq 4, E_{6}, E_{7}$, $E_{8}, F_{4}$ ou $G_{2}$.

- graduada do tipo Cartan: $W(n ; \underline{1}), n \geq 1, S(n ; \underline{1})^{(1)}, n \geq 3, H(2 r ; \underline{1})^{(2)}, r \geq 1$, $K(2 r+1 ; \underline{1})^{(1)}, r \geq 1$.

- a álgebra de Melikian de dimensão 125 (só se $p=5$ ).

Depois da classificação das álgebras de Lie simples e das $p$-álgebras de Lie simples, de dimensão finita, sobre corpos de características $p>3$, o principal problema ainda em aberto na categoria das álgebras de Lie de dimensão finita é a classificação de álgebras de Lie simples e das $p$-álgebras de Lie simples sobre um corpo algebricamente fechado de característica $p=2$ ou $p=3$. O primeiro passo nessa direção foi feito por S. Skryabin, (Ver $[\mathbf{S k}])$. Nesse artigo, S. Skryabin demonstrou o seguinte teorema:

Teorema 0.4 (S. Skryabin, 1998). Seja L uma álgebra de Lie de dimensão finita sobre um corpo $k$ algebricamente fechado de característica 2 e de posto toral absoluto 1 (ver [St1], pág.23, definição 1.2.1). Então L é solúvel. Isto é, não existem álgebras Lie simples de dimensão finita de posto toral absoluto 1 sobre um corpo algebricamente fechado de característica 2.

O Teorema 0.4 é equivalente ao seguinte resultado, cuja demonstração pode ser encontrada em [Gr]:

Teorema 0.5. Seja L uma álgebra de Lie simples de dimensão finita sobre um corpo algebricamente fechado de característica 2. Então L tem posto toral absoluto maior ou igual a 2 . 
A. Grichkov e A. Premet, em [GP], classificaram todas as álgebras de Lie simples de dimensão finita e de posto toral absoluto 2 sobre um corpo algebricamente fechado de característica 2. Esse resultado diz o seguinte:

Teorema 0.6 (Teorema de classificação de Premet-Grichkov). Seja L uma álgebra de Lie simples de dimensão finita sobre um corpo $k$ algebricamente fechado de caracteristica 2. Se o posto toral absoluto de L é 2, então L é clássica de dimensão 3, 8, 14 ou 26.

Como um corolário do Teorema 0.6 temos:

Corolário 0.7. Se $(L,[2])$ é uma 2-álgebra de Lie simples de dimensão finita sobre um corpo $k$, algebricamente fechado de característica 2 e de posto toral 2 , então $L$ é isomorfa a: $\mathfrak{s l}_{3}(k), G_{2}$ ou $D_{4}$.

Um pequeno passo na classificacão das álgebras de Lie simples de dimensão finita de característica 2 é o seguinte resultado (ver [GZ]):

Teorema 0.8 (Grichkov-Zusmanovich, 2016). Seja L uma álgebra de Lie simples de dimensão finita sobre um corpo $k$ algebricamente fechado de característica 2 e de posto toral absoluto 2. Se L contém uma subálgebra de Cartan de posto toral 1, então $L \cong \mathfrak{o}_{3}(k)$.

Para posto toral absoluto ou posto toral maior ou igual que 3 temos os seguintes problemas ainda em aberto:

Problema I. Classificar as álgebras de Lie simples de dimensão finita de posto toral absoluto maior ou igual que 3 sobre um corpo algebricamente fechado de característica 2 .

Problema II. Classificar as 2-álgebras de Lie simples de dimensão finita de posto toral maior ou igual que 3 sobre um corpo algebricamente fechado de característica 2 .

Para o problema I, temos o seguinte resultado devido a M. Guerreiro, A. Grischov e W. Araújo (ver [GGA]), para o caso em que o posto toral absoluto é 3 :

Teorema 0.9 (M. Guerreiro - A. Grichkov - W. Araújo, 2014). Seja L uma álgebra de Lie simples de dimensão 7 e posto toral absoluto 3 sobre um corpo $k$ de característica 2 algebricamente fechado. Então $L$ é isomorfa à álgebra de Witt-Zassenhaus $W(1, \underline{3})^{(1)}$, ou à álgebra de Hamilton $H(2:(2,1) ; \omega)$, onde $\omega:=\left(1+x^{(3)} y\right) d x \wedge d y$.

Até hoje só conhecemos um exemplo concreto de 2-álgebras de Lie simples de posto toral 3 sobre um corpo algebricamente fechado de característica 2 , portanto para o problema II e quando o posto toral é 3, nós propomos a seguinte conjectura:

Conjectura. A única 2-álgebras de Lie simples de posto toral 3 é $W(3, \underline{1})$.

Neste trabalho vamos a dar uma solução parcial para essa conjectura. Portanto o principal objetivo desta tese é o estudo das 2-álgebras de Lie simples de dimensão menor 
ou igual a 17 de posto toral 3 sobre um corpo algebricamente fechado de característica 2 .

Os resultados principais deste trabalho são:

- Não existem 2-álgebras de Lie simples de dimensão menor ou igual a 16 de posto toral 3 sobre um corpo algebricamente fechado de característica 2 (Teorema 2.9).

- Não existem 2-álgebras de Lie $(L,[2])$, simples de dimensão 17 de posto toral 3 sobre um corpo $k$ algebricamente fechado de característica 2 , cuja decomposição de Cartan respeito a subálgebra toral $T$ é: $L:=T \oplus \sum_{\xi \in G} \oplus L_{\xi}$, onde $G:=\langle\alpha, \beta, \gamma\rangle$ é um grupo abeliano elementar de ordem $8, \operatorname{dim}_{k}(T)=3$ e $\operatorname{dim}_{k}\left(L_{\xi}\right) \neq 2$ para algum $\xi \in G$. (Proposição 4.1)

- Não existem 2-álgebras de Lie $(L,[2])$, simples de dimensão 17 de posto toral 3 sobre um corpo $k$ algebricamente fechado de característica 2, contendo uma cópia de $\left(\mathfrak{s l}_{3}(k), 2\right)$, e cuja decomposição de Cartan respeito a subálgebra toral $T$ é: $L:=T \oplus \sum_{\xi \in G} \oplus L_{\xi}$, onde $G:=\langle\alpha, \beta, \gamma\rangle$ é um grupo abeliano elementar de ordem $8, \operatorname{dim}_{k}(T)=3$ e $\operatorname{dim}_{k}\left(L_{\xi}\right)=2$ para todo $\xi \in G$. (Teorema 4.2)

O trabalho está estruturado da seguinte forma (é interessante notar que estaremos trabalhando com álgebras de Lie simples de dimensão finita sobre um corpo $k$ algebricamente fechado de característica 2). No capítulo 1 serão introduzidos os conceitos e a terminologia a serem usados ao longo de todo o trabalho. Vamos a começar o capítulo 1 com algumas definições básicas, por exemplo: 2-álgebras de Lie, posto toral, 2-representações, 2-módulos, decomposição de Cartan, etc. Também apresentaremos um exemplo de uma 2-álgebra de Lie simples de dimensão 34 de posto toral 4 achada por V.G. Kac. e B. Veisfeiler (ver [KV]). Além disso, enunciaremos alguns resultados bem conhecidos e que serão de grande importancia para o trabalho. No capítulo 2 demonstraremos que não existem as 2-álgebras de Lie simples de posto toral 3 de dimensão menor ou igual que 16 sobre um corpo $k$ algebricamente fechado de característica 2 . No capítulo 3, classificaremos os $\mathfrak{s l}_{3}(k)$-2-módulos irredutíveis de dimensão menor ou igual a 8 e, considerando as extensões destes $\mathfrak{s l}_{3}(k)$-2-módulos irredutíveis, apresentaremos uma lista dos $\mathfrak{s l}_{3}(k)$-2-módulos de dimensão menor ou igual a 8 (e, por tabela, em casos em que as extensões cindem, e alguns casos quando as extensões não cindem). Ao final é apresentado o resultado principal deste capítulo que estabelece condições necessarias e suficientes para que exista um $\mathfrak{s l}_{3}(k)$-morfismo $\Lambda^{2}(U) \rightarrow \mathfrak{s l}_{3}(k) \oplus k$, onde $U$ é um $\mathfrak{s l}_{3}(k)$-2-módulo de dimensão 8 . Finalmente, no capítulo 4 , fazendo uso dos resultados do capítulo 1 e 3 , estudaremos a 2-álgebra de Lie simples $(L,[2])$, de dimensão 17 de posto toral 3 e monstraremos em alguns casos especiais que elas não existem. Além disso, apresentamos uma forma de abordar o estudo de $(L,[2])$, quando ela contém uma 2-subálgebra de Lie não simples e não solúvel, e sua decomposição de Cartan respeito a subalgebra toral $T$ é do jeito, $L:=T \oplus \sum_{\xi \in G} \oplus L_{\xi}$ onde $G:=\langle\alpha, \beta, \gamma\rangle$ é um grupo abeliano elementar de ordem $8, \operatorname{dim}_{k}(T)=3$ e $\operatorname{dim}_{k}\left(L_{\xi}\right)=2$ para todo $\xi \in G$. 


\section{Capítulo 1}

\section{Preliminares e Notações}

Neste capítulo estaremos listando alguns definições e resultados básicos que julgamos necessários para a leitura desta tese por serem básicos omitiremos as demonstrações dos resultados, mas estas demonstrações podem ser encontradas em um dos seguintes livros $[\mathrm{SF}],[\mathrm{Ja}]$.

\subsection{Categoria das 2-álgebras de Lie}

Durante todo o texto, nos referiremos a $k$ como sendo um corpo algebricamente fechado de característica 2 contendo o corpo primo $\mathbb{F}_{2}$, e $L$ uma álgebra de Lie sobre $k$ de dimensão finita.

Definição 1.1. ([Ja]). Uma 2-álgebra de Lie é um par $(L,[2])$ onde $L$ é uma álgebra de Lie sobre $k$, e [2] $L \rightarrow L, a \mapsto a^{[2]}$ é uma função (chamada 2-aplicação) tal que:

- $(a+b)^{[2]}=a^{[2]}+b^{[2]}+[a, b], \forall a, b \in L$.

- $(\lambda a)^{[2]}=\lambda^{2} a^{[2]}, \forall \lambda \in k, \forall a \in L$.

- $\operatorname{ad}\left(b^{[2]}\right)=(a d(b))^{2}, \forall b \in L$.

O par $(L,[2])$ é referido como uma 2-álgebra de Lie, e a 2-aplicação [2] é única para qualquer 2-álgebra de Lie $(L,[2]) \operatorname{com} \mathfrak{z}(L)=0$.

Se diz que $(L,[2])$ é uma 2-álgebra de Lie simples, se $(L,[2])$ é uma 2-álgebra de Lie onde $L$ é uma álgebra de Lie simples sobre $k$.

Sejam $\left(L_{1},[2]_{1}\right)$ e $\left(L_{2},[2]_{2}\right)$ 2-álgebras de Lie, $\varphi:\left(L_{1},[2]_{1}\right) \rightarrow\left(L_{2},[2]_{2}\right)$ é um

2-homomorfismo de 2-álgebras de Lie se $\varphi: L_{1} \rightarrow L_{2}$ é um homomorfismo de álgebras de Lie e $\varphi\left(x^{[2]_{1}}\right)=(\varphi(x))^{[2]_{2}}$ para todo $x \in L_{1}$.

Exemplo 1.2. Seja $A$ uma álgebra associativa sobre $k$, e seja $A^{-}$a álgebra de Lie associada a $A$. Defina $x^{[2]}:=x^{2}$, para todo $x \in A$ (chama-se 2-aplicação associativa em A). Então $\left(A^{-}, 2\right)$ é uma 2-álgebra de Lie. Em particular, $\mathfrak{g l}(V):=\operatorname{End}(V)^{-}$, 
onde $V$ é um espaço vetorial de dimensão finita sobre $k$, é uma 2-álgebra Lie, pela 2aplicação associativa. Além disso, qualquer subálgebra de Lie de A, a qual é fechada pela 2-aplicação associativa em A, é uma 2-álgebra de Lie. Portanto, a álgebra das derivações de $L, \operatorname{Der}_{k}(L)$, é uma 2-álgebra de Lie.

Exemplo 1.3. Seja $\mathfrak{s l}_{3}(k):=\left\{a \in \mathfrak{g l}_{3}(k): \operatorname{tr}(a)=0\right\}$. Essa é uma álgebra de Lie simples sobre $k$ de dimensão 8 com operação colchete $[x, y]:=x y-y x$.

$\mathfrak{s l}_{3}(k)$ tem uma base canônica dada por: $\Psi:=\left\{h_{1}, h_{2}, e_{12}, e_{13}, e_{23}, e_{21}, e_{31}, e_{32}\right\}$, onde $h_{1}:=e_{11}+e_{22}, h_{2}:=e_{22}+e_{33}$, geram uma subálgebra de Cartan de $\mathfrak{s l}_{3}(k)$. Assim $\mathfrak{s l}_{3}(k)$ é uma 2-álgebra de Lie, com a 2-aplicação definida como: $a^{[2]}:=a^{2}$ para todo $a \in \Psi$, tal 2-aplicação é única já que $\mathfrak{z}\left(\mathfrak{s l}_{3}(k)\right)=0$. Portanto temos que $\left(\mathfrak{s l}_{3}(k), 2\right)$ é uma 2-álgebra de Lie simples de dimensão 8, e sua multiplicação de Lie é dada pela tabela seguinte :

\begin{tabular}{|c||c|c|c|c|c|c|c|c|}
\hline & $h_{1}$ & $h_{2}$ & $e_{12}$ & $e_{13}$ & $e_{23}$ & $e_{21}$ & $e_{31}$ & $e_{32}$ \\
\hline \hline$h_{1}$ & $h_{1}$ & 0 & 0 & $e_{13}$ & $e_{23}$ & 0 & $e_{31}$ & $e_{32}$ \\
\hline$h_{2}$ & 0 & $h_{2}$ & $e_{12}$ & $e_{13}$ & 0 & $e_{21}$ & $e_{31}$ & 0 \\
\hline$e_{12}$ & 0 & $e_{12}$ & 0 & 0 & $e_{13}$ & $h_{1}$ & $e_{32}$ & 0 \\
\hline$e_{13}$ & $e_{13}$ & $e_{13}$ & 0 & 0 & 0 & $e_{23}$ & $h_{1}+h_{2}$ & $e_{12}$ \\
\hline$e_{23}$ & $e_{23}$ & 0 & $e_{13}$ & 0 & 0 & 0 & $e_{21}$ & $h_{2}$ \\
\hline$e_{21}$ & 0 & $e_{21}$ & $h_{1}$ & $e_{23}$ & 0 & 0 & 0 & $e_{31}$ \\
\hline$e_{31}$ & $e_{31}$ & $e_{31}$ & $e_{32}$ & $h_{1}+h_{2}$ & $e_{21}$ & 0 & 0 & 0 \\
\hline$e_{32}$ & $e_{32}$ & 0 & 0 & $e_{12}$ & $h_{2}$ & $e_{31}$ & 0 & 0 \\
\hline
\end{tabular}

Tabela 1.1: A 2-álgebra de Lie $\left(\mathfrak{s l}_{3}(k), 2\right)$

Os elementos da diagonal são resultados da 2-aplicação nos elementos de suas linhas ou colunas correspondentes.

Exemplo 1.4. Tem-se que: $\mathfrak{g l}_{3}(k)=\mathfrak{s l}_{3}(k) \oplus \mathfrak{z}\left(\mathfrak{g l}_{3}(k)\right)$, onde $\mathfrak{z}\left(\mathfrak{g l}_{3}(k)\right)=k I_{3}$. Com efeito, se $a \in \mathfrak{g l}_{3}(k)$ e $\operatorname{tr}(a)=\lambda$, então $a=\left(a+\lambda I_{3}\right)+\lambda I_{3}$ onde $\left(a+\lambda I_{3}\right) \in \mathfrak{s l}_{3}(k)$ e $\lambda I_{3} \in \mathfrak{z}\left(\mathfrak{g l}_{3}(k)\right)$. Se $a \in \mathfrak{s l}_{3}(k) \cap \mathfrak{z}\left(\mathfrak{g l}_{3}(k)\right)$, então $a=\lambda I_{3}$ e, $0=\operatorname{tr}(a)=\lambda+\lambda+\lambda \operatorname{logo}$ $\lambda=0$, portanto $a=0$.

Exemplo 1.5. Seja $\mathfrak{o}_{3}(k):=\left\{A=\left(a_{i j}\right) \in M_{3}(k): a_{i i}=0\right.$ e $\left.a_{i j}=a_{j i}\right\}$.

$A$ base canônica de $\mathfrak{o}_{3}(k)$ é dada por: $e_{1}:=e_{12}+e_{21}, e_{2}:=e_{13}+e_{31}$, e $e_{3}:=e_{23}+e_{32}$. Então $\mathfrak{o}_{3}(k)=k e_{1} \oplus k e_{2} \oplus k e_{3}, \operatorname{com}\left[e_{1}, e_{2}\right]=e_{3},\left[e_{1}, e_{3}\right]=e_{2},\left[e_{3}, e_{1}\right]=e_{2}$ é a única (a menos de isomorfismo) álgebra de Lie simples de dimensão 3 sobre $k$, mas não é uma 2-álgebra de Lie. De fato, temos que ad $\left(e_{1}\right)^{2}=e_{3}^{2}=e_{22}+e_{33} \neq e_{22}+e_{23}=\operatorname{ad}\left(e_{1}^{2}\right)$. Defina $d \in \operatorname{End}_{k}\left(\mathfrak{o}_{3}(k)\right)$ por:

$$
\begin{array}{ll}
e_{1} & \mapsto a_{12} e_{1} \\
e_{2} & \mapsto a_{13} e_{2} \\
e_{3} & \mapsto a_{23} e_{3}
\end{array}
$$


com $a_{i j} \in k, 1 \leq i<j \leq 3$.

Se $d \in \operatorname{Der}_{k}\left(\mathfrak{o}_{3}(k)\right)$, tem-se, $a_{23} e_{3}=d\left(e_{3}\right)=d\left(\left[e_{1}, e_{2}\right]\right)=\left[d\left(e_{1}\right), e_{2}\right]+\left[e_{1}, d\left(e_{2}\right)\right]=$ $a_{12} e_{3}+a_{13} e_{3}$, portanto $a_{23}=a_{12}+a_{13}$. O mesmo acontece para $d\left(\left[e_{1}, e_{3}\right]\right)$, e $d\left(\left[e_{2}, e_{3}\right]\right)$. Assim, $d \in \operatorname{Der}_{k}\left(\mathfrak{o}_{3}(k)\right)$ se, e só se, $a_{12}+a_{13}+a_{23}=0$. Portanto:

$$
\operatorname{Der}_{k}\left(\mathfrak{o}_{3}(k)\right)=\left\{A=\left(a_{i j}\right) \in M_{3}(k): a_{i j}=a_{j i}, a_{11}+a_{22}+a_{33}=0\right\} .
$$

Uma base para $\operatorname{Der}_{k}\left(\mathfrak{o}_{3}(k)\right)$ é $\left\{h_{1}, h_{2}, e_{1}, e_{2}, e_{3}\right\}$ onde $h_{1}:=e_{1}^{2}=e_{11}+e_{22} e$ $h_{2}:=e_{2}^{2}=e_{11}+e_{33}$ e assim $\operatorname{Der}_{k}\left(\mathfrak{o}_{3}(k)\right):=\operatorname{span}\left\{h_{1}, h_{2}\right\} \oplus \mathfrak{o}_{3}(k)$. Pelo exemplo 1.2 temos que $\operatorname{Der}_{k}\left(\mathfrak{o}_{3}(k)\right)$ é uma 2-álgebra de Lie de dimensão 5 , com multiplicação de Lie dada pela tabela seguinte:

\begin{tabular}{|c||c|c|c|c|c|}
\hline & $h_{1}$ & $h_{2}$ & $e_{1}$ & $e_{2}$ & $e_{3}$ \\
\hline \hline$h_{1}$ & $h_{1}$ & 0 & 0 & $e_{2}$ & $e_{3}$ \\
\hline$h_{2}$ & 0 & $h_{2}$ & $e_{1}$ & 0 & $e_{3}$ \\
\hline$e_{1}$ & 0 & $e_{1}$ & $h_{1}$ & $e_{3}$ & $e_{2}$ \\
\hline$e_{2}$ & $e_{2}$ & 0 & $e_{3}$ & $h_{2}$ & $e_{1}$ \\
\hline$e_{3}$ & $e_{3}$ & $e_{3}$ & $e_{2}$ & $e_{1}$ & $h_{1}+h_{2}$ \\
\hline
\end{tabular}

Tabela 1.2: $\operatorname{Der}_{k}\left(\mathfrak{o}_{3}(k)\right)$

Os elementos da diagonal são resultados da 2-aplicação nos elementos de suas linhas ou colunas correspondentes.

Tem-se que $\mathfrak{o}_{3}(k)_{2} \subset \operatorname{Der}_{k}\left(\mathfrak{o}_{3}(k)\right)$, e como $\operatorname{dim}_{k}\left(\mathfrak{o}_{3}(k)_{2}\right)=5$. Então $\operatorname{Der}_{k}\left(\mathfrak{o}_{3}(k)\right)=$ $\mathfrak{o}_{3}(k)_{2}$. Assim, Der ${ }_{k}\left(\mathfrak{o}_{3}(k)\right)$ é uma 2-álgebra de Lie não solúvel de dimensão 5.

\subsection{Subalgebra toral e posto toral}

Definição 1.6. Seja $(L,[2])$ uma 2-álgebra de Lie.

- Um elemento $x \in(L,[2])$ é chamado semi-simples se $x$ pertence a 2-subálgebra de $(L,[2])$ gerada por $x^{[2]}$, isto é, $x \in \operatorname{span}\left\{x^{[2]}, x^{[2]^{2}} \ldots\right\}$.

- Um elemento $x \in(L,[2])$ é chamado 2-nilpotente se $x^{[2]^{n}}=0$ para algum $n \in \mathbb{N}$.

Teorema 1.7. Decomposição de Jordan-Chevalley-Seligman. Para qualquer $x \in(L,[2])$ existem elementos (únicos) $x_{s}$ e $x_{n}$ em $(L,[2])$ tal que $x_{s}$ é semi-simples, $x_{n}$ é 2-nilpotente e $x=x_{s}+x_{n}$, com $\left[x_{s}, x_{n}\right]=0$.

Um subconjunto $N \subseteq(L,[2])$ é chamado 2-nilpotente se todo $x \in N$ é 2-nilpotente. Se $N$ é uma álgebra de Lie de dimensão finita 2-nilpotente, então pelo teorema de Engel temos que $N$ é nilpotente como álgebra de Lie ordinaria.

Exemplo 1.8. Seja $x:=e_{12}, y:=e_{23}, z:=e_{13} \operatorname{em} \mathfrak{g l}_{3}(k)$, então $N:=\operatorname{span}\{x, y, z\}$ $\operatorname{com}[x, y]=z,[x, z]=[y, z]=0 e, x^{2}=y^{2}=z^{2}=0$ é uma álgebra Lie 2-nilpotente. 
Definição 1.9. Seja $(L,[2])$ uma 2-álgebra de Lie.

- Um elemento $t \in(L,[2])$ é chamado toral, se $t^{[2]}=t$.

- Uma 2-subálgebra $T$ de $(L,[2])$ é chamada toral ou um toro se a 2-aplicação [2] é invertível sobre $T$.

Qualquer subálgebra toral de $(L,[2])$ é abeliana e, por [Ja], teorema 13, pág. 192-193, um toro de $(L,[2])$ admite uma base constituída por elementos torais.

Definição 1.10. [St1] O posto toral de $(L,[2])$ é:

$$
M T(L):=\max \{\operatorname{dim}(T): T \in \Omega(L)\} \text { onde } \Omega(L):=\{T \subseteq L: T \text { é um toro de } L\}
$$

Exemplo 1.11. Seja $L=\mathfrak{s l}_{3}(k)$, como no exemplo 1.3., $T:=\operatorname{span}\left\{h_{1}, h_{2}\right\}$ é uma subálgebra toral de $\mathfrak{s l}_{3}(k)$ e $M T\left(\mathfrak{s l}_{3}(k)\right)=2$.

Teorema 1.12. Seja $(L,[2])$ uma 2-álgebra de Lie e $H$ subálgebra de L. Então $H$ é uma subálgebra de Cartan se, e só se, existe um toro maximal $T \subset L$ tal que $H=A n n_{L}(T)$.

\subsection{Decomposição de Cartan}

Definição 1.13. Seja $V$ um $k$-espaço vetorial.

- Uma representação $\rho_{V}: L \rightarrow \mathfrak{g}(V)$ é chamada 2-representação de $(L,[2])$ em $V$ se $\rho\left(x^{[2]}\right)=\rho(x)^{2}$ para todo $x \in L$.

- Um ( $L,[2])$-módulo ou $L$-2-módulo é um $L$-módulo $V$ tal que $v \cdot x^{[2]}=(v \cdot x) \cdot x$ para todo $v \in V$ e $x \in L$.

Exemplo 1.14. Seja $(L,[2])$ uma 2-álgebra de Lie. Então ad $: L \rightarrow \mathfrak{g l}(L)$ é uma 2 -representação de $(L,[2])$ em $(L,[2])$, denominada representação adjunta.

Seja $T$ um toro maximal de $(L,[2]), H:=A n n_{L}(T)$, e $V$ um $(L,[2])$-módulo de dimensão finita sobre $k$, com $\rho_{V}: L \rightarrow \operatorname{End}(V), \rho_{V}(x)(v):=v \cdot x$, a correspondente 2-representação. Como $T$ é abeliana, então $\rho_{V}(T)$ é abeliana e consiste de elementos semi-simples, $\operatorname{logo} V$ pode-se decompor em espaços pesos respeito a $T$ :

$$
V=\sum_{\lambda \in T^{*}} \oplus V_{\lambda} \text { onde } V_{\lambda}:=\{v \in V: v \cdot t=\lambda(t) v, t \in T\} .
$$

O conjunto de $T$-pesos $\left\{\lambda \in T^{*}: V_{\lambda} \neq 0\right\}$ de $V$ sera denotado por $\Gamma^{\omega}(V, T)$.

Observação 1.15. Se $t$ é um elemento toral de $T$, então $\lambda(t) \in \mathbb{F}_{2}$, com $\lambda \in \Gamma^{\omega}(V, T)$. Com efeito, se $\lambda \in \Gamma^{\omega}(V, T), 0 \neq v \in L_{\lambda} e, t \in T$ tal que $t^{[2]}=t$. Temos:

$$
\lambda(t) v=v \cdot t=v \cdot t^{[2]}=(v \cdot t) \cdot t=(\lambda(t) v) \cdot t=\lambda(t)(v \cdot t)=\lambda(t)^{2} v .
$$

Portanto, $\lambda(t) \in \mathbb{F}_{2}$. 
Em particular, se $V$ é o $(L,[2])$-módulo adjunto, temos que $\Delta:=\Gamma^{\omega}(L, T) \backslash\{0\}$ é o conjunto de sistemas de raízes de $L$ respeito a $T$ e,

$$
L=H \oplus \sum_{\alpha \in \Delta} \oplus L_{\alpha} \quad \text { onde } \quad L_{\alpha}:=\{g \in L: a d(h)(g)=\alpha(h) g, h \in H\}
$$

é o espaço de decomposição de raízes de $(L,[2])$ respeito $H$ ou, a Decomposição de Cartan de $(L,[2])$ respeito a $H$.

Observação 1.16. Se $x \in L_{\alpha}$, então

$$
\operatorname{ad}(x): L_{\beta} \rightarrow L_{\alpha+\beta} \quad e \quad \rho_{V}(x): V_{\beta} \rightarrow V_{\alpha+\beta} .
$$

Com efeito, sejam $x \in L_{\alpha}$ e $v \in V_{\beta}$, então

$$
\begin{gathered}
\rho_{V}(h) \rho_{V}(x)(v)=\rho_{V}([h, x])(v)-\rho_{V}(x) \rho_{V}(h)(v)=\rho(\alpha(h) x)(v)-\rho_{V}(x)(\beta(h) v)= \\
(\alpha+\beta)(h) \rho_{V}(x)(v) .
\end{gathered}
$$

Teorema 1.17. Seja $(L,[2])$ uma 2-álgebra de Lie de dimensão finita sobre $k$ e $H$ subálgebra de Cartan de L. Se $L=H \oplus \sum_{\alpha \in \Delta} \oplus L_{\alpha}$ é a decomposição de Cartan de $L$ respeito a $H$. Então

a. Se $h \in H$ é semi-simples, então adh $\left.\right|_{L_{\alpha}}=\alpha(h) i d_{L_{\alpha}}$.

b. Se $h \in H$ e $h:=h_{n}+h_{s}$ com $h_{n}$ 2-nilpotente e $h_{s}$ semi-simples, então $\alpha(h)=$ $\alpha\left(h_{s}\right)$ para todo $\alpha \in \Delta$.

c. Definindo-se $L_{\alpha}^{[2]}:=\left\{x_{\alpha}^{[2]}: x_{\alpha} \in L_{\alpha}\right\}$, então $L_{\alpha}^{[2]} \subset H$ para todo $\alpha \in \Delta$.

d. $L_{\alpha}^{[2]} \subset \operatorname{Ker}(\alpha)$, para todo $\alpha \in \Delta$.

e. Se L é simples, então $H \subset[L, L]=\sum_{\alpha \in \Delta} \oplus\left[L_{\alpha}, L_{\alpha}\right]$.

A seção a seguir é baseada no artigo [KV], aí vamos apresentar uma 2-álgebra de Lie simples de dimensão 34 e de posto toral 4, que foi descoberta por V. G. Kac e V. Veisfeiler. A estrutura desta $k$-álgebra é semelhante ao que posteriormente vamos a estudar no capítulo 4 , seção 4.1 .

\subsection{Uma 2-álgebra de Lie simples de dimensão 34 de posto toral 4 .}

Suponhamos que $F$ é um corpo algebricamente fechado de característica $p>0$ e $A \in M_{n}(F)$. Denote por $\widetilde{G}(A)$ a álgebra de Lie sobre $F$ definida por:

$$
\widetilde{G}(A) \stackrel{\text { def }}{=} \operatorname{span}\left\{e_{i}, f_{i}, h_{i}: i \in I_{n}\right\}
$$


cujo colchete de Lie está definido pelas seguintes relações $\left(i, j \in I_{n}\right)$ :

$$
\left[e_{i}, f_{j}\right]:=\delta_{i j} h_{i}, \quad\left[h_{i}, h_{j}\right]:=0, \quad\left[h_{i}, e_{j}\right]:=a_{i j} e_{i j}, \quad\left[h_{i}, f_{j}\right]=-a_{i j} f_{j} .
$$

Tem-se que $\widetilde{G}(A)$ é uma $\mathbb{Z}$-álgebra graduada, onde $\operatorname{deg} e_{i}=1, \operatorname{deg} f_{i}=-1$, e $\operatorname{deg} h_{i}=0$, $i \in I_{n}$ e, assim $\widetilde{G}(A)=\sum_{i \in \mathbb{Z}} \oplus \widetilde{G}_{i}(A)$. Seja $J(A)$ o único ideal maximal graduado de $\widetilde{G}(A)$ tal que: $J(A) \cap\left(\widetilde{G}_{-1}(A) \oplus \widetilde{G}_{0}(A) \oplus \widetilde{G}_{1}(A)\right)=0$, onde

$$
\widetilde{G}_{-1}(A) \oplus \widetilde{G}_{0}(A) \oplus \widetilde{G}_{1}(A)=\left(\sum k f_{i}\right) \oplus\left(\sum k h_{j}\right) \oplus\left(\sum k e_{i}\right) .
$$

Então

$$
G(A) \stackrel{\text { def }}{=} \widetilde{G}(A) / J(A) \text { e } G(A) / \mathfrak{z}(G(A))
$$

são chamadas álgebras de Lie congradientes sobre $F$ e $A$ é seu matriz de Cartan.

Teorema 1.18 (Teorema de classificação de V. G. Kac-Weisfeiler, 1970-1991). As álgebras de Lie congradientes simples de dimensão finita sobre um corpo $F$ algebricamente fechado de característica $p \geq 3$ são:

- As clássicas (Ter um pouco de cuidado com $A_{n}, E_{6}$ e, $G_{2}$, ver [KV] pág. 787.)

- Uma familia de álgebras de 1-parametro de dimensão 10, $L(\epsilon)(\epsilon \neq 0)$ consideradas primeiro por A. I. Kostrikin $\left(L(-1)=\mathfrak{s p}_{4}(F)\right)$ em característica $p=3$ :

$$
L(\epsilon)=\left(\mathfrak{s l}_{2}(F) \oplus \mathfrak{s l}_{2}(F)\right) \oplus((2) \otimes(2)) .
$$

- Uma álgebra $\mathfrak{b r}_{29}:=\left(\mathfrak{s l}_{2}(F) \oplus L(1)\right) \oplus((2) \otimes(8))$ de dimensão 29 em característica $p=3$ descuberta em 1982 por Brow. Em 1991, S. M. Skyabin demonstrou que $\mathfrak{b r}_{29} \simeq G(A) \mathrm{com}$

$$
A:=\left(\begin{array}{ccc}
2 & -1 & 0 \\
-1 & 2 & 1 \\
0 & -1 & 0
\end{array}\right)
$$

A classificação das álgebras de Lie congradientes simples de dimensão finita sobre $k$ é ainda um problema em aberto. Em [KV] (pág. 787 e 790) temos uma lista de álgebras de Lie congradientes simples de dimensão finita sobre $k$, essas são:

- Clássicas: $A_{n},(n+1) \not \equiv 0(\bmod 2), A_{2 l-1}^{(1)}, F_{6}, E_{8}, D_{2 n+1} / D_{2 n+1}^{(1)}, E_{7} / E_{7}^{(1)}$, $D_{2 n} / D_{2 n}^{(1)}$

- Não clássicas: $G\left(C_{3, a}\right), G\left(F_{4, a}\right), a \in k \backslash\{0,1\}$ e, $G\left(\Delta_{n}\right), n=1,2, \ldots$ onde :

$$
C_{3, a}:=\left(\begin{array}{ccc}
0 & 1 & 0 \\
a & 0 & 1 \\
0 & 1 & 0
\end{array}\right) \quad F_{4, a}:=\left(\begin{array}{cccc}
a & 0 & 1 & 0 \\
0 & 1 & 0 & 1 \\
0 & 0 & 1 & 0
\end{array}\right)
$$




$$
\Delta_{n}:=\left(\begin{array}{cccccc}
1 & 1 & & & & 0 \\
1 & 0 & & 1 & & \\
& 1 & & 0 & & 1 \\
& & \ddots & & & \\
0 & & & 1 & & 0
\end{array}\right)
$$

Nós estamos interessados em estudar a $G\left(F_{4, a}\right)$.

Tem-se que $G\left(F_{4, a}\right):=\widetilde{G}\left(F_{4, a}\right) / J\left(F_{4, a}\right)$ é uma álgebra de Lie congradiente sobre $k$ com $F_{4, a}, a \neq 0,1$ seu matriz de Cartan, e $J\left(F_{4, a}\right)$ é o único ideal maximal graduado de $G\left(F_{4, a}\right)$ para o qual

$$
\begin{cases}J\left(F_{4, a}\right) \cap\left\{h_{1}, h_{2}, h_{3}, h_{4}\right\} & =0 \\ J\left(F_{4, a}\right) \cap\left\{e_{i}, f_{j}\right\} & =0 .\end{cases}
$$

Pela proposição 3.6, pág. 791 de [KV] temos que $G\left(F_{4, a}\right)$ é uma 2-álgebra de Lie simples de dimensão 34 e,

$$
G\left(F_{4, a}\right) \simeq G\left(F_{4, b}\right) \Longleftrightarrow a=b, \quad a=\frac{1}{b} .
$$

Sejam:

$p_{1}:=e_{1} e_{2}, p_{2}:=e_{1} e_{3}, p_{3}:=e_{1} e_{4}, p_{4}:=e_{2} e_{3}, p_{5}:=e_{2} e_{4}, p_{6}:=e_{3} e_{4}, p_{1} e_{3}:=\left(e_{1} e_{2}\right) e_{3}$, $\left.p_{1} e_{4}:=\left(e_{1} e_{2}\right) e_{4}, p_{2} e_{4}:=\left(e_{1} e_{3}\right) e_{4}, p_{4} e_{4}:=\left(e_{2} e_{3}\right) e_{4}, p:=\left(e_{1} e_{2}\right) e_{3}\right) e_{4}, q_{1}:=f_{1} f_{2}$, $q_{2}:=f_{1} f_{3}, q_{3}:=f_{1} f_{4}, q_{4}:=f_{2} f_{3}, q_{5}:=f_{2} f_{4}, q_{6}:=f_{3} f_{4}, q_{1} f_{3}:=\left(f_{1} f_{2}\right) f_{3}$, $q_{1} f_{4}:=\left(f_{1} f_{2}\right) f_{4}, q_{2} f_{4}:=\left(f_{1} f_{3}\right) f_{4}, p_{4} f_{4}:=\left(f_{2} f_{3}\right) f_{4}$, e $\left.q:=\left(f_{1} f_{2}\right) f_{3}\right) f_{4}$.

Uma base para $\widetilde{G}\left(F_{4, a}\right)$ é:

$$
\begin{aligned}
\Phi:= & \left\{h_{i}\right\}_{i=1}^{4} \cup\left\{e_{i}\right\}_{i=1}^{4} \cup\left\{p_{i}\right\}_{i=1}^{6} \cup\left\{p_{1} e_{3}, p_{1} e_{4}, p_{2} e_{4}, p_{4} e_{4}, p\right\} \cup \\
& \cup\left\{f_{i}\right\}_{i=1}^{4} \cup\left\{q_{i}\right\}_{i=1}^{6} \cup\left\{q_{1} e_{3}, q_{1} e_{4}, q_{2} e_{4}, q_{4} e_{4}, q\right\} .
\end{aligned}
$$

Portanto, uma base de $G\left(F_{4, a}\right)$ é $\left\{\bar{w}:=w+J\left(F_{4, a}\right): w \in \Phi\right\}$. Além disso, temos que: $h_{1}^{[2]}=\delta_{1} h_{1}+\delta_{2} h_{2}+\delta_{3} h_{3}+\delta_{4} h_{4}, \operatorname{logo}$

$0=\left[h_{1},\left[h_{1}, e_{1}\right]\right]=\delta_{1}\left[h_{1}, e_{1}\right]+\delta_{2}\left[h_{2}, e_{1}\right]+\delta_{3}\left[h_{3}, e_{1}\right]+\delta_{4}\left[h_{4}, e_{1}\right]=\delta_{2} a e_{1}$ então $\delta_{2}=0$.

$e_{2}=\left[h_{1},\left[h_{1}, e_{2}\right]\right]=\delta_{1}\left[h_{1}, e_{2}\right]+\delta_{2}\left[h_{2}, e_{2}\right]+\delta_{3}\left[h_{3}, e_{2}\right]+\delta_{4}\left[h_{4}, e_{2}\right]=\delta_{1} e_{2}+\delta_{3} e_{2}$, então $\delta_{1}+\delta_{3}=1$.

$0=\left[h_{1},\left[h_{1}, e_{3}\right]\right]=\delta_{2} e_{3}+\delta_{4} e_{3}$, então $\delta_{2}=\delta_{4}=0$.

$0=\left[h_{1},\left[h_{1}, e_{4}\right]\right]=\delta_{3} e_{4}$, então $\delta_{3}=0$.

Assim, $h_{1}^{[2]}=h_{1}$.

Também temos: $h_{2}^{[2]}=\delta_{1} h_{1}+\delta_{2} h_{2}+\delta_{3} h_{3}+\delta_{4} h_{4}$, logo:

$a^{2} e_{1}=\left[h_{2},\left[h_{2}, e_{1}\right]\right]=\delta_{1}\left[h_{1}, e_{1}\right]+\delta_{2}\left[h_{2}, e_{1}\right]+\delta_{3}\left[h_{3}, e_{1}\right]+\delta_{4}\left[h_{4}, e_{1}\right]$, então $a^{2} e_{1}=\delta_{2} a e_{1}$ portanto, $\delta_{2}=a$. 
$0=\left[h_{2},\left[h_{2}, e_{2}\right]\right]=\left[h_{2}^{[2]}, e_{2}\right]=\delta_{1} e_{2}+\delta_{3} e_{2}$, então $\delta_{1}+\delta_{3}=0$

$e_{3}=\left[h_{2},\left[h_{2}, e_{3}\right]\right]=\delta_{2} e_{3}+\delta_{4} e_{3}$, então $\delta_{4}+\delta_{2}=1$. Assim, $\delta_{4}=1+a:=\bar{a}$.

$0=\left[h_{2},\left[h_{2}, e_{4}\right]\right]=\left[h_{2}^{[2]}, e_{4}\right]=\delta_{3} e_{4}$, então $\delta_{3}=0$

Portanto, $h_{2}^{[2]}=a h_{2}+\bar{a} h_{4}$.

Pelos mesmos argumentos anteriores, encontramos que : $h_{3}^{[2]}=h_{3}, h_{4}^{[2]}=h_{4}$ e, $w^{[2]}=0$, $\forall w \in \Phi \backslash\left\{h_{1}, h_{2}, h_{3}, h_{4}\right\}$. Seja $H:=\operatorname{span}\left\{h_{i}+J\left(F_{4, a}\right): i \in I_{4}\right\}$, tem-se $H$ é uma subálgebra toral de dimensão 4 de $G\left(F_{4, b}\right)$ e, por [Ja], teorema 13, pág. 192-193 existe uma base de $H$ constituída por elementos torais. Procuremos esta base. De fato: seja $t_{2}:=x h_{2}+y h_{4}$, com $x, y \in k$; achemos $x, y$ em $k$ tal que $t_{2}^{[2]}=t_{2}$. Com efeito, $t_{2}^{2}=\left(x h_{2}+y h_{4}\right)^{[2]}=x^{2} h_{2}^{[2]}+x y\left[h_{2}, h_{4}\right]+y^{2} h_{4}^{[2]}=a x^{2} h_{2}+\left(x^{2} \bar{a}+y^{2}\right) h_{4}$, assim

$$
t_{2}^{[2]}=t_{2} \Longleftrightarrow x^{2} a=x \text { e } \bar{a} x^{2}+y^{2}=y .
$$

Logo as soluções $(x, y)$ para o seguinte sistema não linear

$$
\begin{cases}a x^{2}+x & =0 \\ \bar{a} x^{2}+y^{2}+y & =0\end{cases}
$$

em $\mathbb{F}_{2}(a)$ são : $(0,0),(0,1),\left(\frac{1}{a}, \frac{1}{a}\right),\left(\frac{1}{a}, \frac{\bar{a}}{a}\right)$.

- Se a solução é $(0,0)$, então $t_{2}=0$.

- Se a solução é $(0,1)$, então $t_{2}=h_{4}$.

- Se a solução é $\left(\frac{1}{a}, \frac{1}{a}\right)$, então $t_{2}=\frac{1}{a}\left(h_{2}+h_{4}\right)$

- Se a solução é $\left(\frac{1}{a}, \frac{\bar{a}}{a}\right)$, então $t_{2}=\frac{1}{a}\left(h_{2}+\bar{a} h_{4}\right)$

Assim, temos que

$$
T:=\operatorname{span}\left\{t_{i}+J\left(F_{4, a}\right): i \in I_{4}\right\}
$$

onde $t_{i}:=h_{i}, i \in I_{4} \backslash\{2\}$ e, $t_{2} \in\left\{0, h_{4}, \frac{1}{a}\left(h_{2}+h_{4}\right), \frac{1}{a}\left(h_{2}+\bar{a} h_{4}\right)\right\}$ são toros de $G\left(F_{4, a}\right)$ tal que $t_{i}^{[2]}=t_{i}, i=1,2,3,4$. Quando $t_{2}=0$ ou $t_{2}=h_{4}$ temos que $T$ é um toro de dimensão 3. Quando $t_{2}:=\frac{1}{a}\left(h_{2}+h_{4}\right)$ ou $t_{2}=\frac{1}{a}\left(h_{2}+\bar{a} h_{4}\right)$ temos que $\left\{t_{i}+J\left(F_{4, a}\right)\right\}_{i=1}^{4}$ são bases de $H$, Portanto $T=H$, e assim $M T\left(G\left(F_{4, a}\right)\right)=4$.

Agora, procuramos os pesos para cada elemento da base de $\widetilde{G}\left(F_{4, a}\right)$ respeito a

$$
H_{1}=\left\{t_{1}=h_{1}, t_{2}=\frac{1}{a}\left(h_{2}+h_{4}\right), t_{3}=h_{3}, t_{4}=h_{4}\right\} .
$$

Com efeito,

- Para $e_{1}$ :

$$
\begin{aligned}
& {\left[e_{1}, t_{1}\right]=\left[e_{1}, h_{1}\right]=a_{11} e_{1}=0 e_{1}} \\
& {\left[e_{1}, t_{2}\right]=\left[e_{1}, \frac{1}{a}\left(h_{2}+h_{4}\right)\right]=\frac{1}{a}\left(\left[e_{1}, h_{2}\right]+\left[e_{1}, h_{4}\right]=\frac{1}{a}\left(a e_{1}\right)=1 e_{1} .\right.} \\
& {\left[e_{1}, t_{3}\right]=\left[e_{1}, h_{3}\right]=a_{31} e_{1}=0 e_{1}} \\
& {\left[e_{1}, t_{4}\right]=\left[e_{1}, h_{4}\right]=a_{41} e_{1}=0 e_{1}}
\end{aligned}
$$

Logo, o peso de $e_{1}$ é $\beta:=(0,1,0,0)$. 
- Para $e_{2}$ :

$\left[e_{2}, t_{1}\right]=\left[e_{2}, h_{1}\right]=a_{12} e_{2}=1 e_{2}$

$\left[e_{2}, t_{2}\right]=\left[e_{2}, \frac{1}{a}\left(h_{2}+h_{4}\right)\right]=\frac{1}{a}\left(\left[e_{2}, h_{2}\right]+\left[e_{2}, h_{4}\right]=0 e_{2}\right.$.

$\left[e_{2}, t_{3}\right]=\left[e_{2}, h_{3}\right]=a_{32} e_{2}=1 e_{2}$

$\left[e_{2}, t_{4}\right]=\left[e_{1}, h_{4}\right]=a_{41} e_{1}=0 e_{1}$

$\mathrm{O}$ peso de $e_{2}$ é $\alpha+\gamma:=(1,0,1,0)$.

- Para $e_{3}$ :

$\left[e_{3}, t_{1}\right]=\left[e_{3}, h_{1}\right]=a_{13} e_{3}=0 e_{3}$

$\left[e_{3}, t_{2}\right]=\left[e_{3}, \frac{1}{a}\left(h_{2}+h_{4}\right)\right]=\frac{1}{a}\left(\left[e_{3}, h_{2}\right]+\left[e_{3}, h_{4}\right]=0 e_{3}\right.$.

$\left[e_{3}, t_{3}\right]=\left[e_{3}, h_{3}\right]=a_{33} e_{3}=0 e_{3}$

$\left[e_{3}, t_{4}\right]=\left[e_{3}, h_{4}\right]=a_{43} e_{3}=1 e_{3}$

Assim, o peso de $e_{3}$ é $\lambda:=(0,0,0,1)$.

- Para $e_{4}$ :

$$
\begin{aligned}
& {\left[e_{4}, t_{1}\right]=\left[e_{4}, h_{1}\right]=a_{14} e_{4}=0 e_{4}} \\
& {\left[e_{4}, t_{2}\right]=\left[e_{4}, \frac{1}{a}\left(h_{2}+h_{4}\right)\right]=\frac{1}{a}\left(\left[e_{4}, h_{2}\right]+\left[e_{4}, h_{4}\right]=0 e_{4} .\right.} \\
& {\left[e_{4}, t_{3}\right]=\left[e_{4}, h_{3}\right]=a_{34} e_{4}=1 e_{4}} \\
& {\left[e_{4}, t_{4}\right]=\left[e_{4}, h_{4}\right]=a_{44} e_{4}=0 e_{4}} \\
& \text { Logo, o peso de } e_{4} \text { é } \gamma:=(0,0,1,0) .
\end{aligned}
$$

Como $\left[L_{\xi}, L_{\mu}\right] \subseteq L_{\xi+\mu}$ (veja a obs. 1.13) e $\left[h_{i}, f_{j}\right]=-a_{i j} f_{j}$, podemos encontrar os pesos dos elementos restante de $\Phi$. Por exemplo: $e_{1}$ e $e_{2}$ tem peso $\beta$ e $\alpha+\gamma$ respectivamente, então $p_{1}:=e_{1} e_{2}$ tem peso $\beta+(\alpha+\gamma)$, e assim $p_{1} e_{4}$ tem peso $(\alpha+\beta+\gamma)+\gamma=\alpha+\beta$. Portanto, os pesos dos elementos da base de $G\left(F_{4, a}\right)$ são dados na tabela seguinte. 


\begin{tabular}{|l|l|l|}
\hline Espaços raízes & Base & Raízes \\
\hline \hline$L_{\beta}$ & $\overline{e_{1}}, \overline{f_{1}}$ & $\beta:=(0,1,0,0)$ \\
\hline$L_{\alpha+\gamma}$ & $\overline{e_{2}}, \quad \overline{f_{2}}$ & $\alpha+\gamma:=(1,0,1,0)$ \\
\hline$L_{\lambda}$ & $\overline{e_{3}}, \quad \overline{f_{3}}$ & $\lambda:=(0,0,0,1)$ \\
\hline$L_{\gamma}$ & $\overline{e_{4}}, \quad \overline{f_{4}}$ & $\gamma:=(0,0,1,0)$ \\
\hline$L_{\alpha+\beta+\gamma}$ & $\overline{p_{1}}, \overline{q_{1}}$ & $\alpha+\beta+\gamma:=(1,1,1,0)$ \\
\hline$L_{\beta+\lambda}$ & $\overline{p_{2}}, \overline{q_{2}}$ & $\beta+\lambda:=(0,1,0,1)$ \\
\hline$L_{\beta+\gamma}$ & $\overline{p_{3}}, \overline{q_{3}}$ & $\beta+\gamma:=(0,1,1,0)$ \\
\hline$L_{\alpha+\gamma+\lambda}$ & $\overline{p_{4}}, \overline{q_{4}}$ & $\alpha+\gamma+\lambda:=(1,0,1,1)$ \\
\hline$L_{\alpha}$ & $\overline{p_{5}}, \overline{q_{5}}$ & $\alpha:=(1,0,0,0)$ \\
\hline$L_{\gamma+\lambda}$ & $\overline{p_{6}}, \overline{q_{6}}$ & $\gamma+\lambda:=(0,0,1,1)$ \\
\hline$L_{\alpha+\beta+\gamma+\lambda}$ & $\overline{p_{1} \bar{e}_{3}}, \quad q_{1} f_{3}$ & $\alpha+\beta+\gamma+\lambda:=(1,1,1,1)$ \\
\hline$L_{\alpha+\beta}$ & $\bar{p}_{1} \bar{e}_{4}, \quad q_{1} f_{4}$ & $\alpha+\beta:=(1,1,0,0)$ \\
\hline$L_{\beta+\gamma+\lambda}$ & $p_{2} \bar{e}_{4}, \quad q_{2} f_{4}$ & $\beta+\gamma+\lambda:=(0,1,1,1)$ \\
\hline$L_{\alpha+\lambda}$ & $\overline{p_{5}} \bar{e}_{4}, \quad q_{5} f_{4}$ & $\alpha+\lambda:=(1,0,0,1)$ \\
\hline$L_{\alpha+\beta+\lambda}$ & $\overline{p_{1}}, \bar{q}$ & $\alpha+\beta+\lambda:=(1,1,0,1)$ \\
\hline
\end{tabular}

Tabela 1.3: Espaços pesos de $G\left(F_{4, a}\right)$.

Se achamos os pesos dos elementos da base de $\widetilde{G}\left(F_{4, a}\right)$ respeito a

$$
H_{2}=\left\{t_{1}=h_{1}, t_{2}=\frac{1}{a}\left(h_{2}+\bar{a} h_{4}\right), t_{3}=h_{3}, t_{4}=h_{4}\right\}
$$

encontramos que $\Gamma^{\omega}\left(\widetilde{G}\left(F_{4, a}\right), H_{1}\right)=\Gamma^{\omega}\left(\widetilde{G}\left(F_{4, a}\right), H_{2}\right)$. De fato:

$\left[e_{1}, t_{2}\right]=\left[e_{1}, \frac{1}{a}\left(h_{2}+\bar{a} h_{4}\right)\right]=\frac{1}{a}\left[e_{1}, h_{2}\right]+\frac{\bar{a}}{a}\left[e_{1}, h_{4}\right]=\frac{1}{a} a e_{1}=e_{1}$. Logo, o peso de $e_{1}$ é $\beta=(0,1,0,0)$.

$\left[e_{2}, t_{2}\right]=\left[e_{2}, \frac{1}{a}\left(h_{2}+\bar{a} h_{4}\right)\right]=\frac{1}{a}\left[e_{2}, h_{2}\right]+\frac{\bar{a}}{a}\left[e_{2}, h_{4}\right]=0$. Então o peso de $e_{2}$ é $\alpha+\gamma=(1,0,1,0)$.

$\left[e_{3}, t_{2}\right]=\left[e_{3}, \frac{1}{a}\left(h_{2}+\bar{a} h_{4}\right)\right]=\frac{1}{a}\left[e_{3}, h_{2}\right]+\frac{\bar{a}}{a}\left[e_{3}, h_{4}\right]=\frac{1}{a} e_{3}+\frac{\bar{a}}{a} e_{3}=\frac{(1+\bar{a})}{a} e_{3}=e_{3}$. Portanto, o peso de $e_{3}$ é $\beta+\lambda=(0,1,0,1)$.

$\left[e_{4}, t_{2}\right]=\left[e_{4}, \frac{1}{a}\left(h_{2}+\bar{a} h_{4}\right)\right]=\frac{1}{a}\left[e_{4}, h_{2}\right]+\frac{\bar{a}}{a}\left[e_{4}, h_{4}\right]=0$. Assim, o peso de $e_{4}$ é $\gamma=(0,0,1,0)$.

Portanto, tem-se que a decomposição de Cartan de $G\left(F_{4, a}\right)$ respeito a $\bar{H}_{1}:=\operatorname{span}\left\{\bar{t}_{i}: t_{i} \in H_{1}\right\}$ é dada por:

$$
G\left(F_{4, a}\right)=\bar{H}_{1} \oplus \sum_{\xi \in G} \oplus L_{\xi}
$$

onde é um g $G:=\langle\alpha, \beta, \gamma, \lambda\rangle$ grupo abeliano elementar de ordem 16, e $\operatorname{dim}_{k}\left(L_{\xi}\right)=2$ para todo $\xi \in G$. 


\subsection{Extensões de $(L,[2])$-módulos e Lema de Schur.}

No estudo dos módulos, assim como de qualquer outra estrutura algébrica, o procedimento padrão é examinar os submódulos e os módulos quocientes associados. Aparece assim naturalmente o problema de extensão, a saber:

Quais são os $(L,[2])$-módulos $E$ que podemos obter a partir de dois $(L,[2])$-módulos $N$ e $M$ de modo que $N$ seja (isomorfo a) um submódulo de $E$ e $M$ seja isomorfo $E / N$ ? Nessa situação, podemos organizar $N, M$ e $E$ em uma sequência de $(L,[2])$ homomorfismos de módulos

$$
0 \rightarrow N \stackrel{\iota}{\rightarrow} E \stackrel{p}{\rightarrow} M \rightarrow 0
$$

onde a imagem de cada um é o núcleo do próximo; desse modo, $\iota$ é injetora, $i(N)$ é o núcleo de $p$, e $p$ é sobrejetora. Diremos nesse caso que $E$ é uma extensão de $L$-2-módulo de $M$ por $N$, denotada por $M \wedge N$.

Umo dos objetivos deste trabalho é apresentar meios para listar e classificar, em algum sentido, todas as extensões de $M$ por $N$ (de dimensão 8) quando $L=\mathfrak{s l}_{3}(k)$ e, estamos interessados em aquelas extensões de $M$ por $N$ onde $\operatorname{Ext}_{\mathfrak{s l}_{3}(k)}^{1}(M, N)=0$.

Diz-se que uma sequência exata de $L$-módulos $0 \rightarrow M_{1} \stackrel{f}{\rightarrow} M_{2} \stackrel{g}{\rightarrow} M_{3} \rightarrow 0$ cinde se $\operatorname{Im}(f)(=\operatorname{Ker}(g))$ é um somando direto de $M_{2}$. Nesse caso, a sequência dada é equivalente a

$$
0 \rightarrow M_{1} \rightarrow M_{1} \oplus M_{3} \rightarrow M_{3} \rightarrow 0 .
$$

Lema 1.19. Lema de Schur. Se $V$, $U$ são L-módulos irredutíveis e $\varphi: V \rightarrow U$ um morfismo de L-módulos, então ou $\varphi$ é um isomorfismo ou $\varphi \equiv 0$.

\subsection{Potência exterior de módulos}

Sejam $A$ um anel comutativo, $M$ e $N A$-módulos. Lembre que, uma aplicação $r$ linear $f: M^{r} \rightarrow N$ chama-se alternada se $f\left(m_{1}, m_{2}, \ldots, m_{r}\right)=0$ sempre que $m_{i}=m_{j}$ para algum $i \neq j$.

Seja $J_{r}(r \geq 2)$ o $A$-submódulo do $A$-módulo $M^{\otimes^{r}}$ gerado por todos os elementos de tipo $m_{1} \otimes m_{2} \otimes \ldots \otimes m_{r}$ com $m_{i}=m_{j}$ para algum $i \neq j$, ou seja,

$$
J_{r}:=\left\langle m_{1} \otimes m_{2} \otimes \ldots \otimes m_{r}:\left(\left(m_{1}, \ldots, m_{r}\right),(i, j)\right) \in M^{r} \times I_{r} ; i \neq j, m_{i}=m_{j}\right\rangle .
$$

Define-se o $A$-módulo quociente

$$
\Lambda^{r}(M):=M^{\otimes^{r}} / J_{r}
$$

e chama-se a potência exterior r-ésima de $M$.

Seja $p: M^{\otimes^{r}} \rightarrow M^{\otimes^{r}} / J_{r}$ o $A$-morfismo quociente. Para todo $m_{1}, \ldots, m_{r} \in M$ a clase $p\left(m_{1}, \ldots, m_{r}\right)$ em $\Lambda^{r}(M)$ é denotada por $m_{1} \wedge m_{2} \wedge \ldots \wedge m_{r}$. Por conveniência, poremos 
$\Lambda^{0}(M):=A$ e $, \Lambda^{1}(M):=M\left(\operatorname{assim}, J_{0}=J_{1}=0.\right)$

Para $r \geq 2$, a aplicação canônica $M^{r} \stackrel{\otimes}{\rightarrow} M^{\otimes^{r}}$ é $r$-linear, logo a composição $\wedge:=p \circ \otimes$ é uma aplicação $r$-linear alternada.

Teorema 1.20. Propriedade Universal da potência exterior.

Para toda aplicação $r$-linear alternada $f: M^{r} \rightarrow N$, existe uma única aplicação A-linear $\widetilde{f}: \Lambda^{r}(M) \rightarrow N$ tal que $\tilde{f} \circ \wedge=f$.

Exemplo 1.21. Segunda potência exterior $\Lambda^{2}(M)$.

1. $\Lambda^{2}(M)=M \otimes_{A} M / J_{2}$, onde $J_{2}=\langle m \otimes m: m \in M\rangle$.

2. Se $m, n \in M$ então em $\Lambda^{2}(M)$ temos $m \wedge m=m \otimes m+J_{2}=0$ e $m \wedge n=-n \wedge m$.

3. Existe uma única aplicação A-linear $\Lambda^{2}(M) \stackrel{\alpha_{2, M}}{\longrightarrow} M^{\otimes^{2}}$ tal que

$$
m_{1} \wedge m_{2} \mapsto \sum_{\sigma \in S_{2}}(\operatorname{sign\sigma }) m_{\sigma(1)} \otimes m_{\sigma(2)}=m_{1} \otimes m_{2}-m_{2} \otimes m_{1}
$$

De fato, seja $f: M^{2} \rightarrow M^{\otimes^{2}}$ dada por $f\left(m_{1}, m_{2}\right):=m_{1} \otimes m_{2}-m_{2} \otimes m_{1} . \quad f$ é uma aplicação bilinear e $f(m, m)=0$ logo pela teorema 1.20 existe uma única aplicação A-linear $\Lambda^{2}(M) \stackrel{\alpha_{2, M}}{\longrightarrow} M^{\otimes^{2}}$ tal que $\alpha_{2, M} \circ \wedge=f$.

Observação 1.22. Quando $M=V$ é um k-espaço vetorial de dimensão finita $\Lambda^{2}(V) \stackrel{\alpha_{2, V}}{\longrightarrow} V^{\otimes^{2}}$ é injetora. Isso significa que podemos considerar a $\Lambda^{2}(V)$ como um subespaço de $V \otimes_{k} V$.

4. Se $M$ é um A-módulo livre de posto $d$ com base $e_{1}, \ldots, e_{d}$ então, $\Lambda^{2}(M)$ é um $A$-módulo livre de posto $\left(\begin{array}{l}d \\ 2\end{array}\right)$ com base $\left\{e_{i} \wedge e_{j}: 1 \leq i<j \leq d\right\}$.

5. Para quaisquer A-módulos livres $M, N$ tem-se o isomorfismo de A-módulos:

$$
\Lambda^{2}(M \oplus N) \simeq \sum_{p+q=2} \oplus\left(\Lambda^{p}(M) \otimes_{A} \Lambda^{q}(N)\right) \simeq \Lambda^{2}(M) \oplus\left(M \otimes_{A} N\right) \oplus \Lambda^{2}(N) .
$$

Sejam $V$ e $U L$-módulos. Então existe uma única estrutura de $L$-módulo em $U \otimes_{k} V$ tal que

$$
(u \otimes v) \cdot x:=(u \cdot x) \otimes v+u \otimes(v \cdot x) . \quad(\forall x \in L, u \in U, v \in V) .
$$

Agora se $U$ e $V$ são $(L,[2])$-módulos, então $U \otimes_{k} V$ é também um objeto da categoria dos $(L,[2])$-módulos. De fato, para $x \in L, u \in U$ e $v \in V$ temos:

$$
\begin{aligned}
(u \otimes v) \cdot x^{[2]} & =\left(u \cdot x^{[2]}\right) \otimes v+u \otimes\left(v \cdot x^{[2]}\right) \\
& =((u \cdot x) \cdot x) \otimes v+u \otimes((v \cdot x) \cdot x) \\
& =((u \cdot x) \cdot x) \otimes v+(u \cdot x) \otimes(v \cdot x)+(u \cdot x) \otimes(v \cdot x)+u \otimes((v \cdot x) \cdot x) \\
& =((u \cdot x) \otimes v) \cdot x+(u \otimes(v \cdot x)) \cdot x=((u \cdot x) \otimes v+u \otimes(v \cdot x)) \cdot x=((u \otimes v) \cdot x) \cdot x .
\end{aligned}
$$

Portanto, se $V$ é um $k$-espaço vetorial de dimensão finita então $\Lambda^{2}(V)$ é um $(L,[2])$-submódulo do $(L,[2])$-módulo $V \otimes_{k} V$. 


\section{Capítulo 2}

\section{As 2-álgebras de Lie simples de $\operatorname{dim}_{k} \leq 16$ de posto toral 3}

Neste capítulo, vamos demonstrar que não existem 2-álgebras de Lie simples, de dimensão menor ou igual a 16, e de posto toral 3.

\subsection{As 2-álgebras de Lie simples de $\operatorname{dim}_{k} \leq 6$}

Teorema 2.1. Seja $\mathfrak{g}$ uma álgebra de Lie simples sobre um corpo $F$ algebricamente fechado de característica $p>0$. Suponhamos que $\operatorname{dim}_{F}(\mathfrak{g}) \leq 6$. Então $\mathfrak{g}$ é de dimensão 3 ou, $p=5$ e $\mathfrak{g} \simeq W(1, \underline{1})$.

Demonstração. Ver teorema $2.2 \mathrm{em}[\mathbf{S t}]$.

Suponhamos que existe uma 2-álgebra de Lie $(L,[2])$ simples de $\operatorname{dim}_{k}(L) \leq 6$. Em particular, $L$ é uma álgebra de Lie simples sobre $k$ de $\operatorname{dim}_{k}(L) \leq 6$; além do mais, a característica de $k$ é $p=2$ e, $L$ não é isomorfa $W(1, \underline{1})$ (caso contrário, se $L \simeq W(1, \underline{1})$ então $W(1, \underline{1})$ é simples sobre $k$, o qual é absurdo). Então, pelo teorema 2.1, temos que $L$ é de dimensão 3 , assim $L \simeq \mathfrak{o}_{3}(k)$. Porém, pelo exemple $1.5, \mathfrak{o}_{3}(k)$ não é uma 2-álgebra de Lie. Portanto temos:

Corolário 2.2. Não existem 2-álgebras de Lie simples de $\operatorname{dim}_{k} \leq 6$.

\subsection{Decomposição de Cartan de uma 2-álgebra de Lie de posto toral 3.}

A descomposição de Cartan é uma ferramenta poderosa no estudo das álgebras de Lie. Portanto, vamos começar como no caso clássico, isto é, construindo a decomposição de Cartan de $(L,[2])$ respeito a uma subálgebra toral $T$ de dimensão 3.

Suponhamos que $(L,[2])$ é uma 2-álgebra de Lie simples de $\operatorname{dim}_{k}(L) \geq 7$ e $M T(L)=3$. Logo $L$ contém un toro maximal $T$ de $\operatorname{dim}_{k}(T)=M T(L)=3$. 
Como $\operatorname{ad}(T)$ é abeliana e consiste de elementos semisimples, $L$ pode-se decompor em espaços de pesos respeito a $T$, isto é:

$$
L=A n n_{L}(T) \oplus \sum_{\lambda \in T^{*} \backslash\{0\}} \oplus L_{\lambda} \quad \text { onde, } \quad L_{\lambda}=\{v \in L:[t, v]=\lambda(t) v, \forall t \in T\}
$$

Agora, dado que $T$ é um toro de dimensão 3, por [Ja], Teorema 13, pág. 192-193, existe uma base $\left\{t_{i} \in T: t_{i}{ }^{2}=t_{i}, i=1,2,3\right\}$ de $T$ tal que $\lambda\left(t_{i}\right) \in \mathbb{F}_{2}$. Portanto para qualquer $\lambda \in \operatorname{End}\left(T, \mathbb{F}_{2}\right)$ temos que $\lambda:=\left(\lambda_{1}, \lambda_{2}, \lambda_{3}\right) \in \mathbb{F}_{2}^{3}, \operatorname{com} \lambda_{i} \in \mathbb{F}_{2}$ e

$$
T^{*}:=\{(0,0,0),(1,0,0),(0,1,0),(0,0,1),(1,1,0),(1,0,1),(0,1,1),(1,1,1)\} .
$$

Sejam $\alpha:=(1,0,0), \beta:=(1,0,0)$ e $\gamma:=(0,0,1), \log \{\alpha \alpha, \beta, \gamma\}$ é uma base de $T^{*}$. Além disso, seja $H:=A n n_{L}(T)$. Então pelo Teorema 1.12, $H$ é uma subalgebra de Cartan de $L$, e assim, pelo Teorema 1.7 temos que $H=T \oplus N$, onde $N$ é uma subálgebra 2-nilpotente de $L$ e $[T, N]=0$. Portanto temos que a decomposição em espaços de pesos de $L$ respeito a $T$ é:

$$
L=T \oplus N \oplus L_{\alpha} \oplus L_{\beta} \oplus L_{\gamma} \oplus L_{\alpha+\beta} \oplus L_{\alpha+\gamma} \oplus L_{\beta+\gamma} \oplus L_{\alpha+\beta+\gamma} .
$$

Daquí para frente, vamos supor que existe uma 2-álgebra de Lie simples, $(L ;[2])$, o qual contém uma subálgebra toral maximal $T$ de $\operatorname{dim}(T)=M T(L)=3$, cuja decomposição em espaços de pesos respeito a $T$ é:

$$
L=T \oplus N \oplus L_{\alpha} \oplus L_{\beta} \oplus L_{\gamma} \oplus L_{\alpha+\beta} \oplus L_{\alpha+\gamma} \oplus L_{\beta+\gamma} \oplus L_{\alpha+\beta+\gamma} .
$$

$\mathrm{Ou}$

$$
L=H \oplus \sum_{\xi \in G} \oplus L_{\xi},
$$

onde $G:=\langle\alpha, \beta, \gamma\rangle$ é um grupo abeliano elementar de ordem 8.

Proposição 2.3. Existem três raizes em $T^{*} \backslash\{0\}$ linearmente independentes, isto é, existem $\xi, \delta, \theta$ em $T^{*}$ linearmente independentes tais que $L_{\xi} \neq 0, L_{\delta} \neq 0$ e $L_{\theta} \neq 0$

Demonstração. Suponha que a proposição é falsa. Então temos os seguintes casos:

Caso I. Existem $\xi, \delta$ em $T^{*} \backslash\{0\}$ linearmente independentes tais que $L_{\xi} \neq 0$ e $L_{\delta} \neq 0$, ou

Caso II. Existe $\xi \in T^{*} \backslash\{0\}$ tal que $L_{\xi} \neq 0$.

No caso I, podemos estender $\{\xi, \delta\} \subseteq T^{*} \backslash\{0\}$ a uma base de $T^{*}$, logo existe $\lambda \in T^{*} \backslash\{0\}$ tal que $\{\xi, \delta, \lambda\} \subseteq T^{*} \backslash\{0\}$ é um conjunto linearmente independente e $L_{\lambda}=0$. Pela ação do grupo $G L_{3}\left(\mathbb{F}_{2}\right)$ sobre $T^{*}$ podemos considerar a seguinte mudança de base dada por:

$$
\begin{array}{rll}
G L_{3}\left(\mathbb{F}_{2}\right) \times T^{*} & \stackrel{\psi_{A}}{\longrightarrow} & T^{*} \\
(A, \xi) & \mapsto & A . \xi=\alpha \\
(A, \delta) & \mapsto & A . \delta:=\beta \\
(A, \lambda) & \mapsto & A . \lambda:=\gamma
\end{array}
$$




\subsection{DECOMPOSIÇÃO DE CARTAN DE UMA 2-ÁLGEBRA DE LIE DE POSTO TORAL 3.23}

Assim, encontramos que $L=H \oplus L_{\alpha} \oplus L_{\beta} \oplus L_{\alpha+\beta}$. Consequentemente, salvo mudança de base, $L$ tem a forma

$$
L=H \oplus L_{\varepsilon} \oplus L_{\mu} \oplus L_{\varepsilon+\mu},
$$

onde $\{\varepsilon, \mu, \mu+\varepsilon\}$ é um conjunto linearmente dependiente de $T^{*}$. Neste caso, $L$ tem posto toral 2, o qual é uma contradição.

Para o caso II, estendendo $\{\xi\} \subseteq T^{*} \backslash\{0\}$ a uma base de $T^{*}$, temos que existem $\pi, \kappa \in$ $T^{*} \backslash\{0\}$ tais que $\{\xi, \pi, \kappa\}$ é um conjunto linearmente independente tal que $L_{\xi} \neq 0$ e $L_{\pi}=L_{\kappa}=0$.

Logo, pela ação de $G L_{3}\left(\mathbb{F}_{2}\right)$ sobre $T^{*}$, podemos fazer uma mudança de base, isto é, para $A$ fixo em $G L_{3}\left(\mathbb{F}_{2}\right)$, temos:

$$
\begin{array}{rll}
G L_{3}\left(\mathbb{F}_{2}\right) \times T^{*} & \stackrel{\psi_{A}}{\longrightarrow} T^{*} \\
(A, \xi) & \mapsto A . \xi=\alpha \\
(A, \pi) & \mapsto A . \pi:=\beta \\
(A, \kappa) & \mapsto & A . \kappa:=\gamma .
\end{array}
$$

Portanto, salvo mudança de base $L$ tem a forma seguinte:

$$
L=H \oplus L_{\varepsilon}
$$

para algum $\varepsilon \in T^{*} \backslash\{0\}$. Nesse caso temos que $L$ tem posto toral 1. Uma contradição. Portanto existem três pesos em $T$ linearmente independentes tais que cada espaço peso associado a elas não são nulos.

Agora, se existe $\delta \in\{\alpha, \beta, \gamma\}$ tal que $L_{\delta}=0$, então consideremos a mudança de base

$$
\begin{array}{rll}
G L_{3}\left(\mathbb{F}_{2}\right) \times T^{*} & \stackrel{\psi_{A}}{\longrightarrow} T^{*} \\
(A, \alpha) & \mapsto & A . \alpha=\kappa \\
(A, \beta) & \mapsto & A . \beta:=\mu \\
(A, \gamma) & \mapsto & A . \gamma:=\lambda .
\end{array}
$$

Como $\{\kappa, \mu, \lambda\}$ são linearmente independentes, então pela Proposicão $2.3, L_{\kappa} \neq 0$, $L_{\mu} \neq 0$, e $L_{\lambda} \neq 0$, logo a decomposição de $L$ fica da forma seguinte:

$$
L=H \oplus L_{\kappa} \oplus L_{\mu} \oplus L_{\lambda} \oplus L_{\kappa+\mu} \oplus L_{\kappa+\lambda} \oplus L_{\mu+\lambda} \oplus L_{\kappa+\mu+\lambda} .
$$

Assim, pode-se supor sem perda de generalidade que a decomposição em espaços de pesos de $L$ é:

$$
L=T \oplus N \oplus L_{\alpha} \oplus L_{\beta} \oplus L_{\gamma} \oplus L_{\alpha+\beta} \oplus L_{\alpha+\gamma} \oplus L_{\beta+\gamma} \oplus L_{\alpha+\beta+\gamma},
$$

onde $\{\alpha, \beta, \gamma\}$ é uma base de $T^{*}$ tal que $L_{\alpha} \neq 0, L_{\beta} \neq 0$, e $L_{\gamma} \neq 0$. Suponhamos que

$$
\operatorname{dim}_{k}\left(L_{\alpha}\right) \geq \operatorname{dim}_{k}\left(L_{\beta}\right) \geq \operatorname{dim}_{k}\left(L_{\gamma}\right) \geq \operatorname{dim}_{k}\left(L_{\xi}\right)>0, \quad \forall \xi \in T^{*} \backslash\{\alpha, \beta, \alpha+\beta\} .
$$

Seja $\Delta:=\left\{\lambda \in T^{*}: L_{\lambda} \neq 0\right\}$ o sistema de raízes $L$ respeito a $T$, encontremos todos os $\Delta \subseteq T^{*}$ de tal forma que em todos os casos $\alpha, \beta$ e $\gamma \in \Delta$. Logo temos os seguintes casos: 
1. Se $\operatorname{Card}(\Delta)=3$, temos $\Delta_{1}=\{\alpha, \beta, \gamma\}$.

2. Se $\operatorname{Card}(\Delta)=4$, temos as seguintes possibilidades para $\Delta$ :

$$
\begin{aligned}
& \Delta_{2}=\{\alpha, \beta, \gamma, \alpha+\beta\} \\
& \Delta_{3}=\{\alpha, \beta, \gamma, \alpha+\gamma\} \\
& \Delta_{4}=\{\alpha, \beta, \gamma, \beta+\gamma\} \\
& \Delta_{5}=\{\alpha, \beta, \gamma, \alpha+\beta+\gamma\}
\end{aligned}
$$

3. Se $\operatorname{Card}(\Delta)=5$, temos a seguintes possibilidades para $\Delta$ :

$$
\begin{aligned}
& \Delta_{6}=\{\alpha, \beta, \gamma, \alpha+\beta, \alpha+\gamma\} \\
& \Delta_{7}=\{\alpha, \beta, \gamma, \alpha+\beta, \beta+\gamma\} \\
& \Delta_{8}=\{\alpha, \beta, \gamma, \alpha+\beta, \alpha+\beta+\gamma\} \\
& \Delta_{9}=\{\alpha, \beta, \gamma, \alpha+\gamma, \beta+\gamma\} \\
& \Delta_{10}=\{\alpha, \beta, \gamma, \alpha+\gamma, \alpha+\beta+\gamma\} \\
& \Delta_{11}=\{\alpha, \beta, \gamma, \beta+\gamma, \alpha+\beta+\gamma\}
\end{aligned}
$$

4. Se $\operatorname{Card}(\Delta)=6$, tem-se as seguintes possibilidades para $\Delta$ :

$$
\begin{aligned}
& \Delta_{12}=\{\alpha, \beta, \gamma, \alpha+\beta, \alpha+\gamma, \beta+\gamma\} \\
& \Delta_{13}=\{\alpha, \beta, \gamma, \alpha+\beta, \alpha+\gamma, \alpha+\beta+\gamma\} \\
& \Delta_{14}=\{\alpha, \beta, \gamma, \alpha+\beta, \beta+\gamma, \alpha+\beta+\gamma\} \\
& \Delta_{15}=\{\alpha, \beta, \gamma, \alpha+\gamma, \beta+\gamma, \alpha+\beta+\gamma\}
\end{aligned}
$$

5. Se $\operatorname{Car}(\Delta)=7$, então $\Delta_{0}=T^{*} \backslash\{0\}$

Assim que, para cada $\Delta_{i}$ com $0 \leq i \leq 15$, temos uma Decomposição de Cartan de $(L,[2])$ respeito a $H$ dada por:

$$
L^{\Delta_{i}}=T \oplus N \oplus \sum_{\xi \in \Delta_{i}} \oplus L_{\xi}
$$

Fixando $\operatorname{dim}_{k}\left(L^{\Delta_{i}}\right)$ estudaremos a decomposição de Cartan de cada $L^{\Delta_{i}}$, e os detalhes sobre a estrutura fornecida pela decomposição de Cartan destas álgebras nos darão as ferramentas necessarias para sua classificação. Para esta finalidade, precisamos demonstrar as seguintes proposicões.

Proposição 2.4. Sejam $\alpha, \beta \in \Delta \backslash\{0\}$. Se existe $e_{\alpha} \in L_{\alpha}$ tal que $e_{\alpha}^{[2]}:=t_{\alpha}+n_{\alpha}$, $t_{\alpha} \in T \backslash\{0\}, n_{\alpha} \in N$ e $\beta\left(t_{\alpha}\right) \neq 0$, então $L_{\beta} \simeq L_{\alpha+\beta}$. 
Demonstração. Seja $e_{\alpha} \in L_{\alpha}$ e consideremos $\operatorname{ad}\left(e_{\alpha}\right): L_{\beta} \rightarrow L_{\alpha+\beta}$ a aplicação linear adjunta. Vejamos que $a d\left(e_{\alpha}\right)$ é injetora. Sejam $e_{\beta}, f_{\beta}$ em $L_{\beta}$ tais que $a d\left(e_{\alpha}\right)\left(e_{\beta}\right)=$ $\operatorname{ad}\left(e_{\alpha}\right)\left(f_{\beta}\right)$. Então $\left[e_{\alpha}, e_{\beta}\right]=\left[e_{\alpha}, f_{\beta}\right] . \operatorname{Logo}\left[e_{\alpha}^{[2]}, e_{\beta}\right]=\left[e_{\alpha},\left[e_{\alpha}, e_{\beta}\right]\right]=\left[e_{\alpha},\left[e_{\alpha}, f_{\beta}\right]\right]=$ $\left[e_{\alpha}{ }^{[2]}, f_{\beta}\right]$. Daí $\left[t_{\alpha}+n_{\alpha}, e_{\beta}\right]=\left[t_{\alpha}+n_{\alpha}, f_{\beta}\right]$, isto é,

$\beta\left(t_{\alpha}\right) e_{\beta}+a d\left(n_{\alpha}\right)\left(e_{\beta}\right)=\beta\left(t_{\alpha}\right) f_{\beta}+a d\left(n_{\alpha}\right)\left(f_{\beta}\right)$, isto é, $\beta\left(t_{\alpha}\right)\left(e_{\beta}+f_{\beta}\right)=a d\left(n_{\alpha}\right)\left(e_{\beta}+f_{\beta}\right)$. Portanto, $a d\left(t_{\alpha}\right)\left(e_{\beta}+f_{\beta}\right)=a d\left(n_{\alpha}\right)\left(e_{\beta}+f_{\beta}\right)$. Como $a d\left(n_{\alpha}\right)$ é nilpotente, existe $m \in \mathbb{N}$ tal que $a d\left(n_{\alpha}\right)^{m}=0$. Assim $a d\left(t_{\alpha}\right)^{m}\left(e_{\beta}+f_{\beta}\right)=0$ e portanto $\left(a d\left(t_{\alpha}\right)\left(e_{\beta}+f_{\beta}\right)\right)^{m}=0$. Logo $a d\left(t_{\alpha}\right)\left(e_{\beta}+f_{\beta}\right)=0$ e daí $\beta\left(t_{\alpha}\right)\left(e_{\beta}+f_{\beta}\right)=0$. Como $\beta\left(t_{\alpha}\right) \neq 0$ temos $e_{\beta}=f_{\beta}$, assim $\operatorname{ad}\left(e_{\alpha}\right)$ é injetora.

Por outro lado, temos que $a d\left(e_{\alpha}\right): L_{\alpha+\beta} \rightarrow L_{\beta}$ é uma aplicação linear injetora, já que: $(\alpha+\beta)\left(t_{\alpha}\right)=\alpha\left(t_{\alpha}\right)+\beta\left(t_{\alpha}\right)=0+\beta\left(t_{\alpha}\right)=\beta\left(t_{\alpha}\right) \neq 0$. Portanto:

$\operatorname{dim}_{k}\left(L_{\beta}\right)=\operatorname{dim}_{k} \operatorname{Ker}\left(\operatorname{ad}\left(e_{\alpha}\right)\right)+\operatorname{dim}_{k} \operatorname{Im}\left(\operatorname{ad}\left(e_{\alpha}\right)\right)=\operatorname{dim}_{k} \operatorname{Im}\left(\operatorname{ad}\left(e_{\alpha}\right)\right) \leq \operatorname{dim}_{k}\left(L_{\alpha+\beta}\right), \mathrm{e}$ $\operatorname{dim}_{k}\left(L_{\alpha+\beta}\right)=\operatorname{dim}_{k} \operatorname{Ker}\left(\operatorname{ad}\left(e_{\alpha}\right)\right)+\operatorname{dim}_{k} \operatorname{Im}\left(\operatorname{ad}\left(e_{\alpha}\right)\right)=\operatorname{dim}_{k} \operatorname{Im}\left(\operatorname{ad}\left(e_{\alpha}\right)\right) \leq \operatorname{dim}_{k}\left(L_{\beta}\right)$. Assim, $\operatorname{dim}_{k}\left(L_{\beta}\right)=\operatorname{dim}_{k}\left(L_{\alpha+\beta}\right)$.

Lema 2.5. Seja $H$ uma subálgebra de Cartan com posto toral maximal. Então $H \oplus L_{\xi}$ é uma subálgebra solúvel de L para todo $\xi$.

Demonstração. Ver [GP].

Observação 2.6. Neste trabalho vamos supor que $H$ é uma subálgebra de Cartan de $L$ de posto toral maximal.

Proposição 2.7. Seja $L=H \oplus \sum_{\xi \in \Delta} \oplus L_{\xi}$. Então $\left[L_{\xi}, L_{\xi}\right] \subseteq \operatorname{Ker}(\xi)$ para todo $\xi \in \Delta$.

Demonstração. Seja $\xi \in \Delta$ fixo. Temos pelo lema 2.5 que $S_{\xi}:=H \oplus L_{\xi}$ é uma subálgebra solúvel de $L$. Vejamos que $\left[L_{\xi}, L_{\xi}\right] \subseteq \operatorname{ker}(\xi)$. Suponhamos que existe $h \in\left[L_{\xi}, L_{\xi}\right]$ tal que $\xi(h) \neq 0$. Dado que $L_{\xi} \subseteq S_{\xi}$, então $\left[L_{\xi}, L_{\xi}\right] \subseteq\left[S_{\xi}, S_{\xi}\right]=S_{\xi}^{(1)}$, logo $h \in S_{\xi}^{(1)}$. Assim $\left[h, S_{\xi}\right] \subseteq S_{\xi}^{(1)}$ e, como $\xi(h) \neq 0$, tem-se $L_{\xi} \subseteq S_{\xi}^{(1)}$. Portanto $h \in\left[L_{\xi}, L_{\xi}\right] \subseteq$ $\left[S_{\xi}^{(1)}, S_{\xi}^{(1)}\right]=S_{\xi}^{(2)}$.

Agora, suponhamos que $h \in S_{\xi}^{(m)}$ para algum $m \in \mathbb{N}$. Como $S_{\xi}^{(m)}$ é um ideal de $S_{\xi}$, então $\left[h, S_{\xi}\right] \subseteq S_{\xi}^{(m)}$, portanto $L_{\xi} \subseteq S_{\xi}^{(m)}$ (ja que $\xi(t) \neq 0$ ). Então $h \in\left[L_{\xi}, L_{\xi}\right] \subseteq$ $\left[S_{\xi}^{(m)}, S_{\xi}^{(m)}\right]=S_{\xi}^{(m+1)}$, assim $h \in S_{\xi}^{(m+1)}$. Logo por indução, $h \in S_{\xi}^{(m)}$ para todo $m$, e como $S_{\xi}$ é solúvel, então $h=0$. Isto implica que $\xi(h)=0$. Uma contradição. Portanto $\xi\left(\left[L_{\xi}, L_{\xi}\right]\right)=0$.

\subsection{Análise de $L=H \oplus \sum_{\xi \in \Delta_{i}} \oplus L_{\xi}, \operatorname{com} \operatorname{card}\left(\Delta_{i}\right)<7$.}

Nesta seção vamos demonstrar que para cada $\Delta_{i}, 1 \leq i \leq 15$, a decomposição de Cartan de $L, L^{\Delta_{i}}=H \oplus \sum_{\xi \in \Delta_{i}} \oplus L_{\xi}$, associada a cada sistema de raízes $\Delta_{i}$, geram contradições com as condições impostas a $(L,[2])$. Portanto, essas incompatibilidades permitem concluir que não existe uma 2-álgebra de Lie simples de dimensão finita de posto toral 3 com estas estruturas. 
Se $\operatorname{dim}_{k}\left(L_{\xi}\right)=1$ para todo $\xi \in \Delta_{i} 1 \leq i \leq 15$, então temos que $0 \neq I:=N \oplus \sum_{\xi \in \Delta_{i}} \oplus L_{\xi}$ é um ideal não trivial de $L$, ja que $\left[N, L_{\xi}\right]=0=\left[L_{\xi}, L_{\xi}\right]$, o qual é uma contradição, já que $L$ é uma álgebra de Lie simples. Assim, nos casos seguintes vamos a supor que $\operatorname{dim}_{k}\left(L_{\xi}\right) \geq 2$ para todo $\xi \in \Delta_{i}$.

- 2.2.1. Se $\Delta_{1}=\{\alpha, \beta, \gamma\}$, temos que: $L=T \oplus N \oplus L_{\alpha} \oplus L_{\beta} \oplus L_{\gamma}$.

Demonstremos que $\left[L_{\xi}, L_{\xi}\right] \subseteq N$, para todo $\xi \in \Delta_{1}$. Com efeito, sejam $e_{i}^{\xi}$ e $e_{j}^{\xi}$ elementos da base de $L_{\xi}$, então $x^{\xi}:=\left[e_{i}^{\xi}, e_{j}^{\xi}\right] \in\left[L_{\xi}, L_{\xi}\right] \subseteq H=T \oplus N$. Logo existem $t_{i j}^{\xi} \in T$ e $n_{i j}^{\xi} \in N$ tal que $: x^{\xi}=t_{i j}^{\xi}+n_{i j}^{\xi}=\alpha\left(t_{i j}^{\xi}\right) t_{1}+\beta\left(t_{i j}^{\xi}\right) t_{2}+\gamma\left(t_{i j}^{\xi}\right) t_{3}+n_{i j}^{\xi}$ pela proposição 2.7 temos $0=\xi\left(x^{\xi}\right)=\xi\left(t_{i j}^{\xi}+n_{i j}^{\xi}\right)$ para todo $\xi \in \Delta_{1}$, e pelo teorema 1.17.b obtemos $0=\xi\left(x^{\xi}\right)=\xi\left(t_{i j}^{\xi}+n_{i j}^{\xi}\right)=\xi\left(t_{i j}^{\xi}\right) . \operatorname{Logo} \alpha\left(t_{i j}^{\alpha}\right)=\beta\left(t_{i j}^{\beta}\right)=\gamma\left(t_{i j}^{\gamma}\right)=0$, portanto $x^{\xi}=n_{i j}^{\xi} \in N$ para todo $\xi \in \Delta_{1}$. Assim, pelo teorema 1.17.e temos $H=\sum_{\xi \in \Delta_{1}}\left[L_{\xi}, L_{\xi}\right] \subseteq N$, então $H=N$ e $T=0$. Uma contradição.

- 2.2 .2 .

2.2.2.a. Se $\Delta_{2}=\{\alpha, \beta, \gamma, \alpha+\beta\}$, então $L=T \oplus N \oplus L_{\alpha} \oplus L_{\beta} \oplus L_{\gamma} \oplus L_{\alpha+\beta}$.

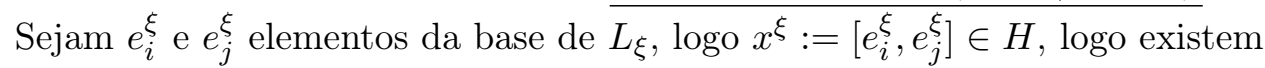
$t_{i j}^{\xi} \in T$ e $n_{i j}^{\xi} \in N$ tal que: $x^{\xi}=t_{i j}^{\xi}+n_{i j}^{\xi}$, assim pela proposição $2.7 \mathrm{e}$ teorema 1.17.b, $0=\xi\left(x^{\xi}\right)=\xi\left(t_{i j}^{\xi}+n_{i j}^{\xi}\right)=\xi\left(t_{i j}^{\xi}\right)$ para todo $\xi \in \Delta_{2}$, isto é : $\alpha\left(t_{i j}^{\alpha}\right)=\beta\left(t_{i j}^{\beta}\right)=\gamma\left(t_{i j}^{\gamma}\right)=(\alpha+\beta)\left(t_{i j}^{\alpha+\beta}\right)=0$. Por outro lado, pela identidade de Jacobi e como $\alpha+\gamma \notin \Delta_{2}$, temos que para todo $e_{k}^{\gamma} \in L_{\gamma}$ : $0=\left[e_{k}^{\gamma},\left[e_{i}^{\alpha}, e_{j}^{\alpha}\right]\right]=\left[e_{k}^{\gamma}, x^{\alpha}\right]=\left[e_{k}^{\gamma}, t_{i j}^{\alpha}+n_{i j}^{\alpha}\right]=\left[e_{k}^{\gamma}, t_{i j}^{\alpha}\right]+\left[e_{k}^{\gamma}, n_{i j}^{\alpha}\right]=\gamma\left(t_{i j}^{\alpha}\right) e_{k}^{\gamma}+$ $\operatorname{ad}\left(n_{i j}^{\alpha}\right)\left(e_{k}^{\gamma}\right)$, assim $\left.\operatorname{ad}\left(n_{i j}^{\alpha}\right)\right|_{L_{\gamma}}=\left.\gamma\left(t_{i j}^{\alpha}\right)\right|_{L_{\gamma}}$. Como $a d\left(n_{i j}^{\alpha}\right)$ é nilpotente, existe $m \in \mathbb{N}$ tal que $a d\left(n_{i j}^{\alpha}\right)^{m}=0, \operatorname{logo} \gamma\left(t_{i j}^{\alpha}\right)=0$. Portanto: $x^{\alpha}=t_{i j}^{\alpha}+n_{i j}^{\alpha}=$ $\alpha\left(t_{i j}^{\alpha}\right) t_{1}+\beta\left(t_{i j}^{\alpha}\right) t_{2}+\gamma\left(t_{i j}^{\alpha}\right) t_{3}+n_{i j}^{\alpha}=\beta\left(t_{i j}^{\alpha}\right) t_{2}+n_{i j}^{\alpha}$.

Assim, $\left[L_{\alpha}, L_{\alpha}\right] \subseteq\left\langle t_{2}\right\rangle \oplus N$.

Da mesma forma, pela identidade de Jacobi, e como $\alpha+\gamma, \beta+\gamma, \alpha+\beta+\gamma \notin \Delta_{2}$ obtemos: $\alpha\left(t_{i j}^{\gamma}\right)=\beta\left(t_{i j}^{\gamma}\right)=\gamma\left(t_{i j}^{\beta}\right)=\gamma\left(t_{i j}^{\alpha+\beta}\right)=0$. Portanto:

$x^{\beta}=t_{i j}^{\beta}+n_{i j}^{\beta}=\alpha\left(t_{i j}^{\beta}\right) t_{1}+n_{i j}^{\beta} ; x^{\gamma}=t_{i j}^{\gamma}+n_{i j}^{\gamma}=n_{i j}^{\gamma} ;$

$x^{\alpha+\beta}=t_{i j}^{\alpha+\beta}+n_{i j}^{\alpha+\beta}=\alpha\left(t_{i j}^{\alpha+\beta}\right) t_{1}+\beta\left(t_{i j}^{\alpha+\beta}\right) t_{2}+n_{i j}^{\alpha+\beta} \operatorname{com}, \alpha\left(t_{i j}^{\alpha+\beta}\right)=\beta\left(t_{i j}^{\alpha+\beta}\right)$. Assim: $\left[L_{\beta}, L_{\beta}\right] \subseteq\left\langle t_{1}\right\rangle \oplus N ;\left[L_{\gamma}, L_{\gamma}\right] \subseteq N$ e, $\left[L_{\alpha+\beta+\gamma}, L_{\alpha+\beta+\gamma}\right] \subseteq\left\langle t_{1}+t_{2}\right\rangle \oplus$ $N$.

Como $L$ é simples, temos $H=\sum_{\xi \in \Delta_{2}}\left[L_{\xi}, L_{\xi}\right] \subseteq\left\langle t_{1}, t_{2}\right\rangle \oplus N$, então $\operatorname{dim}_{k}(T) \leq 2$. Uma contradição.

2.2.2.b. Se $\Delta_{3}=\{\alpha, \beta, \gamma, \alpha+\gamma\}$, então $L=T \oplus N \oplus L_{\alpha} \oplus L_{\beta} \oplus L_{\gamma} \oplus L_{\alpha+\gamma}$.

Pelo mesmo argumento do caso anterior, e como $\alpha+\beta, \beta+\gamma$, e $\alpha+\beta+\gamma$ não estám em $\Delta_{3}$ encontramos que: $x^{\alpha}=\gamma\left(t_{i j}^{\alpha}\right) t_{3}+n_{i j}^{\alpha} ; x^{\beta}=n_{i j}^{\beta} ; x^{\gamma}=$ $=\alpha\left(t_{i j}^{\gamma}\right) t_{1}+n_{i j}^{\gamma} ; x^{\alpha+\gamma}=\alpha\left(t_{i j}^{\alpha+\gamma}\right) t_{1}+\gamma\left(t_{i j}^{\alpha+\gamma}\right) t_{3}+n_{i j}^{\alpha+\gamma} \operatorname{com} \alpha\left(t_{i j}^{\alpha+\gamma}\right)=\gamma\left(t_{i j}^{\alpha+\gamma}\right)$. Assim que: $\left[L_{\alpha}, L_{\alpha}\right] \subseteq\left\langle t_{3}\right\rangle \oplus N ;\left[L_{\beta}, L_{\beta}\right] \subseteq N ;\left[L_{\gamma}, L_{\gamma}\right] \subseteq\left\langle t_{1}\right\rangle \oplus N$; 
$\left[L_{\alpha+\gamma}, L_{\alpha+\gamma}\right] \subseteq\left\langle t_{1}+t_{3}\right\rangle \oplus N$. Portanto:

$H=\sum_{\xi \in \Delta_{3}}\left[L_{\xi}, L_{\xi}\right] \subseteq\left\langle t_{1}, t_{3}\right\rangle \oplus N$, assim $\operatorname{dim}_{k}(T) \leq 2$. Uma contradição.

2.2.2.c. Se $\Delta_{4}=\{\alpha, \beta, \gamma, \beta+\gamma\}$, então $L=T \oplus N \oplus L_{\alpha} \oplus L_{\beta} \oplus L_{\gamma} \oplus L_{\beta+\gamma}$.

Como $\alpha+\beta, \alpha+\gamma, \alpha+\beta+\gamma \notin \Delta_{4}$ pelo mesmo raciocínio dado em

2.2.2.a obtemos:

$x^{\alpha}=n_{i j}^{\alpha} ; x^{\beta}=t_{i j}^{\beta}+n_{i j}^{\beta}=\beta\left(t_{i j}^{\beta}\right) t_{3}+n_{i j}^{\beta} ; x^{\gamma}=t_{i j}^{\gamma}+n_{i j}^{\gamma}=\beta\left(t_{i j}^{\gamma}\right) t_{2}+n_{i j}^{\gamma}$. $x^{\beta+\gamma}=t_{i j}^{\beta+\gamma}+n_{i j}^{\beta+\gamma}=\beta\left(t_{i j}^{\beta+\gamma}\right) t_{2}+\gamma\left(t_{i j}^{\beta \gamma}\right) t_{3}+n_{i j}^{\beta+\gamma}, \operatorname{com} \beta\left(t_{i j}^{\beta+\gamma}\right)=\gamma\left(t_{i j}^{\beta+\gamma}\right)$. Portanto: $\left[L_{\alpha}, L_{\alpha}\right] \subseteq N ;\left[L_{\beta}, L_{\beta}\right] \subseteq\left\langle t_{3}\right\rangle \oplus N ;\left[L_{\gamma}, L_{\gamma}\right] \subseteq\left\langle t_{2}\right\rangle \oplus N$;

$\left[L_{\beta+\gamma}, L_{\beta+\gamma}\right] \subseteq\left\langle t_{2}+t_{3}\right\rangle \oplus N$. Assim $H=\sum_{\xi \in \Delta_{4}}\left[L_{\xi}, L_{\xi}\right] \subseteq\left\langle t_{2}, t_{3}\right\rangle \oplus N, \log \mathrm{O}$ $\operatorname{dim}_{k}(T) \leq 2$. Uma contradição.

2.2.2.d. Se $\Delta_{5}=\{\alpha, \beta, \gamma, \alpha+\beta+\gamma\}$, então $L=T \oplus N \oplus L_{\alpha} \oplus L_{\beta} \oplus L_{\gamma} \oplus L_{\alpha+\beta+\gamma}$ temos que $\alpha+\beta, \alpha+\gamma, \beta+\gamma \notin \Delta_{5}$ então:

$\left[L_{\alpha}, L_{\alpha}\right],\left[L_{\beta}, L_{\beta}\right],\left[L_{\gamma}, L_{\gamma}\right],\left[L_{\alpha+\beta+\gamma}, L_{\alpha+\beta+\gamma}\right] \subseteq N$. Portanto $H \subseteq N$, assim que $T=0$. Uma contradição.

- 2.2 .3 .

2.2.3.a Quando $\Delta_{6}=\{\alpha, \beta, \gamma, \alpha+\beta, \alpha+\gamma\}$, temos

$$
\underline{L}=T \oplus N \oplus L_{\alpha} \oplus L_{\beta} \oplus L_{\gamma} \oplus L_{\alpha+\beta} \oplus L_{\alpha+\gamma} .
$$

Seja $e_{i}^{\xi}, e_{j}^{\xi}$ elementos da base de $L_{\xi}, \operatorname{com} \xi \in \Delta_{6}$, então $x^{\xi}:=\left[e_{i}^{\xi}, e_{j}^{\xi}\right] \in$ $\in\left[L_{\xi}, L_{\xi}\right] \subseteq H=T \oplus N$, logo existem $t_{i j}^{\xi} \in T$ e $n_{i j}^{\xi} \in N$ tal que: $x^{\xi}=t_{i j}^{\xi}+n_{i j}^{\xi}=\alpha\left(t_{i j}^{\xi}\right) t_{1}+\beta\left(t_{i j}^{\xi}\right) t_{2}+\gamma\left(t_{i j}^{\xi}\right) t_{3}+n_{i j}^{\xi}$, pela proposição $2.7 \mathrm{e}$ teorema 1.17.b, obtemos $0=\xi\left(x^{\xi}\right)=\xi\left(t_{i j}^{\xi}+n_{i j}^{\xi}\right)=\xi\left(t_{i j}^{\xi}\right), \forall \xi \in \Delta_{6}$. Logo $\alpha\left(t_{i j}^{\alpha}\right)=\beta\left(t_{i j}^{\beta}\right)=\gamma\left(t_{i j}^{\gamma}\right)=(\alpha+\beta)\left(t_{i j}^{\alpha+\beta}\right)=(\alpha+\gamma)\left(t_{i j}^{\alpha+\gamma}\right)=0$. Como $\beta+\gamma, \alpha+\beta+\gamma \notin \Delta_{6}$ temos que: $x^{\alpha}=\beta\left(t_{i j}^{\alpha}\right) t_{2}+\gamma\left(t_{i j}^{\alpha}\right) t_{3}+n_{i j}^{\alpha} \operatorname{com} \beta\left(t_{i j}^{\alpha}\right)=$ $\gamma\left(t_{i j}^{\alpha}\right) ; x^{\beta}=t_{i j}^{\beta}+n_{i j}^{\beta}=\alpha\left(t_{i j}^{\beta}\right) t_{1}+\gamma\left(t_{i j}^{\beta}\right) t_{3}+n_{i j}^{\beta} \operatorname{com} \alpha\left(t_{i j}^{\beta}\right)=\gamma\left(t_{i j}^{\beta}\right), \operatorname{logo}$ $0=\left[e_{\gamma}, x^{\beta}\right]=\left[e_{\gamma}, t_{i j}^{\beta}\right]+\left[e_{\gamma}, n_{i j}^{\beta}\right]=\gamma\left(t_{i j}^{\beta}\right) e_{\gamma}+\left[e_{\gamma}, n_{i j}^{\beta}\right]$, argumentando como em 2.2.a encontramos que $\gamma\left(t_{i j}^{\beta}\right)=0$, assim $x^{\beta}=n_{i j}^{\beta}$.

$x^{\gamma}=\alpha\left(t_{i j}^{\gamma}\right) t_{1}+\beta\left(t_{i j}^{\gamma}\right) t_{2}+n_{i j}^{\gamma} \operatorname{com} \alpha\left(t_{i j}^{\gamma}\right)=\beta\left(t_{i j}^{\gamma}\right), \operatorname{logo} 0=\left[e_{\beta}, x^{\gamma}\right]=$ $=\left[e_{\beta}, t_{i j}^{\gamma}\right]+\left[e_{\beta}, n_{i j}^{\gamma}\right]=\beta\left(t_{i j}^{\gamma}\right) e_{\beta}+\left[e_{\gamma}, n_{i j}^{\gamma}\right]$ então $\beta\left(t_{i j}^{\gamma}\right)=0$, portanto, $x^{\gamma}=n_{i j}^{\gamma}$. $x^{\alpha+\beta}=t_{i j}^{\alpha+\beta}+n_{i j}^{\alpha+\beta}=\alpha\left(t_{i j}^{\alpha+\beta}\right) t_{1}+\beta\left(t_{i j}^{\alpha+\beta}\right) t_{2}+n_{i j}^{\alpha+\beta} \operatorname{com} \alpha\left(t_{i j}^{\alpha+\beta}\right)=\beta\left(t_{i j}^{\alpha+\beta}\right)$ e $\gamma\left(t_{i j}^{\alpha+\beta}\right)=0$, como $0=\left[e_{\alpha+\gamma}, x^{\alpha+\beta}\right]$, então $\alpha\left(t_{i j}^{\alpha+\beta}\right)=0$, logo $x^{\alpha+\beta}=n_{i j}^{\alpha+\beta}$. $x^{\alpha+\gamma}=t_{i j}^{\alpha+\gamma}+n_{i j}^{\alpha+\gamma}=\alpha\left(t_{i j}^{\alpha+\gamma}\right) t_{1}+\gamma\left(t_{i j}^{\alpha+\gamma}\right) t_{3}+n_{i j}^{\alpha+\gamma} \operatorname{com} \alpha\left(t_{i j}^{\alpha+\gamma}\right)=\gamma\left(t_{i j}^{\alpha+\gamma}\right)$ e $\beta\left(t_{i j}^{\alpha+\gamma}\right)=0$, como $0=\left[e_{\alpha+\beta}, x^{\alpha+\gamma}\right]$, então $\alpha\left(t_{i j}^{\alpha+\gamma}\right)=0$, logo $x^{\alpha+\gamma}=n_{i j}^{\alpha+\gamma}$.

Portanto obtemos que: $\left[L_{\xi}, L_{\xi}\right] \subseteq N$ para todo $\xi \in \Delta_{6} \backslash\{\alpha\}$

e, $\left[L_{\alpha}, L_{\alpha}\right] \subseteq\left\langle t_{2}+t_{3}\right\rangle \oplus N$, portanto

$H=\sum_{\xi \in \Delta_{6}}\left[L_{\xi}, L_{\xi}\right] \subseteq\left\langle t_{2}, t_{3}\right\rangle \oplus N, \operatorname{logo} \operatorname{dim}_{k}(T) \leq 2$. Uma contradição.

2.2.3.b. Se $\Delta_{7}=\{\alpha, \beta, \gamma, \alpha+\beta, \beta+\gamma\}$ temos que:

$$
L=T \oplus N \oplus L_{\alpha} \oplus L_{\beta} \oplus L_{\gamma} \oplus L_{\alpha+\beta} \oplus L \beta+\gamma .
$$


Como no caso precedente, se $x^{\xi}:=\left[e_{i}^{\xi}, e_{j}^{\xi}\right] \in\left[L_{\xi}, L_{\xi}\right] \subseteq H$, então $\xi\left(x^{\xi}\right)=0$, $\forall \xi \in \Delta_{7}, \operatorname{assim} \alpha\left(t_{i j}^{\alpha}\right)=\beta\left(t_{i j}^{\beta}\right)=\gamma\left(t_{i j}^{\gamma}\right)=(\alpha+\beta)\left(t_{i j}^{\alpha+\beta}\right)=$ $=(\beta+\gamma)\left(t_{i j}^{\beta+\gamma}\right)=0$. Por outro lado, como $\alpha+\gamma, \alpha+\beta+\gamma \notin \Delta_{7}$ temos que: $x^{\alpha}=\beta\left(t_{i j}^{\alpha}\right) t_{2}+n_{i j}^{\alpha}$ e assim $\left[e_{\beta+\gamma}, x^{\alpha}\right]=\left[e_{\beta+\gamma}, t_{i j}^{\alpha}\right]+\left[e_{\beta+\gamma}, n_{i j}^{\alpha}\right]$, então $0=(\beta+\gamma)\left(t_{i j}^{\alpha+\beta}\right)=0$. Também $0=\gamma\left(t_{i j}^{\alpha}\right) e_{\gamma}+\left[e_{\gamma}, n_{i j}^{\alpha}\right]$, então $0=\gamma\left(t_{i j}^{\alpha}\right)$ $\operatorname{logo} \beta\left(t_{i j}^{\alpha}\right)=0$, assim que $x^{\alpha}=n_{i j}^{\alpha}$. Como $x^{\beta}=\alpha\left(t_{i j}^{\beta}\right) t_{1}+\gamma\left(t_{i j}^{\beta}\right) t_{3}+n_{i j}^{\beta}$, e $(\alpha+\gamma) \notin \Delta_{7}$ então $\alpha\left(t_{i j}^{\beta}\right)=\gamma\left(t_{i j}^{\beta}\right)$. Agora, $x^{\gamma}=\alpha\left(t_{i j}^{\gamma}\right) t_{1}+\beta\left(t_{i j}^{\gamma}\right) t_{2}+n_{i j}^{\gamma}$, logo $0=\left[e_{\alpha}, x^{\gamma}\right]=\left[e_{\alpha}, t_{i j}^{\gamma}\right]+\left[e_{\alpha}, n_{i j}^{\gamma}\right]$, então $\alpha\left(t_{i j}^{\gamma}\right)=0$. Também $\left[e_{\alpha+\beta}, x^{\gamma}\right]=$ $\left[e_{\alpha+\beta}, t_{i j}^{\alpha+\beta}\right]+\left[e_{\alpha+\beta}, n_{i j}^{\alpha+\beta}\right]$ então $(\alpha+\beta)\left(t_{i j}^{\alpha+\beta}\right)=0$, isto é $\beta\left(t_{i j}^{\alpha+\beta}\right)=0$. Assim, $x^{\gamma}=n_{i j}^{\gamma}$. Além disso, $x^{\alpha+\beta}=\alpha\left(t_{i j}^{\alpha+\beta}\right) t_{1}+\beta\left(t_{i j}^{\alpha+\beta}\right) t_{2}+n_{i j}^{\alpha+\beta}$ com $\alpha\left(t_{i j}^{\alpha+\beta}\right)=\beta\left(t_{i j}^{\alpha+\beta}\right)$.

$x^{\beta+\gamma}=\beta\left(t_{i j}^{\beta+\gamma}\right) t_{2}+\gamma\left(t_{i j}^{\beta+\gamma}\right) t_{3}+n_{i j}^{\beta+\gamma} \operatorname{com} \beta\left(t_{i j}^{\beta+\gamma}\right)=\gamma\left(t_{i j}^{\beta+\gamma}\right)$.

Portanto: $\left[L_{\alpha}, L_{\alpha}\right],\left[L_{\gamma}, L_{\gamma}\right] \subseteq N ;\left[L_{\beta}, L_{\beta}\right] \subseteq\left\langle t_{1}+t_{3}\right\rangle \oplus N$; $\left[L_{\alpha+\beta}, L_{\alpha+\beta}\right] \subseteq\left\langle t_{1}+t_{2}\right\rangle \oplus N ;\left[L_{\beta+\gamma}, L_{\beta+\gamma}\right] \subseteq\left\langle t_{2}+t_{3}\right\rangle \oplus N$.

Seja $T_{0}=k\left\{t_{1}+t_{3}\right\} \oplus k\left\{t_{1}+t_{2}\right\} \oplus k\left\{t_{2}+t_{3}\right\}$, logo

$H \subseteq[L, L] \subseteq \sum_{\xi \in \Delta_{7}}\left[L_{\xi}, L_{\xi}\right] \subseteq T_{0} \oplus N$.

Afirmação. $t_{1} \notin[L, L]$. De fato, se $t_{1} \notin[L, L]$, então existem $\delta_{1}, \delta_{2}, \delta_{3}$ em $k$ e, $n \in N$ tal que $t_{1}=\delta_{1}\left(t_{1}+t_{3}\right)+\delta_{2}\left(t_{1}+t_{3}\right)+\delta_{3}\left(t_{2}+t_{3}\right)+n$, isto é, $\left(1+\delta_{1}+\delta_{2}\right) t_{1}+\left(\delta_{2}+\delta_{3}\right) t_{2}+\left(\delta_{1}+\delta_{3}\right) t_{3}=n$, como $T \cap N=0$ então $\left(1+\delta_{1}+\delta_{2}\right) t_{1}+\left(\delta_{2}+\delta_{3}\right) t_{2}+\left(\delta_{1}+\delta_{3}\right) t_{3}=0$. Como $t_{1}, t_{2}, t_{3}$ são linearmente independente, tem-se o seguinte sistema de equações:

$$
\begin{cases}1+\delta_{1}+\delta_{2} & =0 \\ \delta_{2}+\delta_{3} & =0 \\ \delta_{1}+\delta_{3} & =0\end{cases}
$$

Resolvendo o sistema, temos que $0 \delta_{1}+0 \delta_{2}=1$, o que não pode ocorrer, e assim $t_{1} \notin[L, L]$. Mas a afirmação é falsa, já que $T \subseteq[L, L]$. Uma contradição.

2.2.3.c. $\Delta_{8}=\{\alpha, \beta, \gamma, \alpha+\beta, \alpha+\beta+\gamma\}$ temos que:

$$
\underline{L}=T \oplus N \oplus L_{\alpha} \oplus L_{\beta} \oplus L_{\gamma} \oplus L_{\alpha+\beta} \oplus L_{\alpha+\beta+\gamma} .
$$

$x^{\alpha}=t_{i j}^{\alpha}+n_{i j}^{\alpha}$ então $\alpha\left(t_{i j}^{\alpha}\right)=0$ e, como $\alpha+\gamma \notin \Delta_{8}$, então $\gamma\left(t_{i j}^{\alpha}\right)=0$. Agora $0=\left[e_{\alpha+\beta+\gamma}, x^{\alpha}\right]=\left[e_{\alpha+\beta+\gamma}, t_{i j}^{\alpha}\right]+\left[e_{\alpha+\beta+\gamma}, n_{i j}^{\alpha}\right] \operatorname{logo}(\alpha+\beta+\gamma)\left(t_{i j}^{\alpha}\right)=0$ $\operatorname{assim} \beta\left(t_{i j}^{\alpha}\right)=0$ então $x^{\alpha}=n_{i j}^{\alpha}$. Também,

$x^{\beta}=t_{i j}^{\beta}+n_{i j}^{\beta}$ então $\beta\left(t_{i j}^{\beta}\right)=0$ e como $\beta+\gamma \notin \Delta_{8}$ então $\gamma\left(t_{i j}^{\beta}\right)=0$. Agora $0=\left[e_{\alpha+\beta+\gamma}, x^{\beta}\right]=\left[e_{\alpha+\beta+\gamma}, t_{i j}^{\beta}\right]+\left[e_{\alpha+\beta+\gamma}, n_{i j}^{\beta}\right] \operatorname{logo}(\alpha+\beta+\gamma)\left(t_{i j}^{\beta}\right)=0$ assim $\alpha\left(t_{i j}^{\beta}\right)=0$, então $x^{\beta}=n_{i j}^{\beta}$. Agora, $x^{\gamma}=t_{i j}^{\gamma}+n_{i j}^{\gamma}$ então $\gamma\left(t_{i j}^{\gamma}\right)=0$ e como $\beta+\gamma, \alpha+\gamma \notin \Delta_{8}$ então $\beta\left(t_{i j}^{\gamma}\right)=0$ e $\alpha\left(t_{i j}^{\gamma}\right)=0$. Além do mais, se 
$x^{\alpha+\beta+\gamma}=t_{i j}^{\alpha+\beta+\gamma}+n_{i j}^{\alpha+\beta+\gamma}$ então $(\alpha+\beta+\gamma)\left(t_{i j}^{\alpha+\beta+\gamma}\right)=0 \mathrm{e}$, como

$\beta+\gamma, \alpha+\gamma \notin \Delta_{8}$ então $\beta\left(t_{i j}^{\alpha+\beta+\gamma}\right)=0$ e $\alpha\left(t_{i j}^{\alpha+\beta+\gamma}\right)=0$. Assim que $x^{\alpha+\beta+\gamma}=n_{i j}^{\alpha+\beta+\gamma}$. Por outro lado,

$x^{\alpha+\beta}=t_{i j}^{\alpha+\beta}+n_{i j}^{\alpha+\beta}$, então $(\alpha+\beta)\left(t_{i j}^{\alpha+\beta}\right)=0 \operatorname{logo}$

$x^{\alpha+\beta}=\alpha\left(t_{i j}^{\alpha+\beta}\right) t_{1}+\beta\left(t_{i j}^{\alpha+\beta}\right) t_{2}+\gamma\left(t_{i j}^{\alpha+\beta}\right) t_{3}+n_{i j}^{\alpha+\beta} \operatorname{com} \beta\left(t_{i j}^{\alpha+\beta}\right)=\alpha\left(t_{i j}^{\alpha+\beta}\right)$.

Assim, $\left[L_{\xi}, L_{\xi}\right] \subseteq N$ para todo $\xi \in \Delta_{8} \backslash\{\alpha+\beta\} \mathrm{e}$,

$\left[L_{\alpha+\beta}, L_{\alpha+\beta}\right] \subseteq\left\langle t_{1}+t_{2}, t_{3}\right\rangle \oplus N$. Logo $H \subseteq\left\langle t_{1}+t_{2}, t_{3}\right\rangle \oplus N$, assim

$\operatorname{dim}_{k}(H) \leq \operatorname{dim}_{k}(N)+2$, então $\operatorname{dim}_{k}(T) \leq 2$. Uma contradição.

2.2.3.d. Se $\Delta_{9}=\{\alpha, \beta, \gamma, \alpha+\gamma, \beta+\gamma\}$, então

$$
\underline{L=T \oplus N \oplus L_{\alpha} \oplus L_{\beta} \oplus L_{\gamma} \oplus L_{\alpha+\gamma} \oplus L_{\beta+\gamma} .}
$$

Temos que $x^{\alpha}=t_{i j}^{\alpha}+n_{i j}^{\alpha}$, então $\alpha\left(t_{i j}^{\alpha}\right)=0$ e como $(\alpha+\beta) \notin \Delta_{9}$ então $0=\left[e_{\beta}, x^{\alpha}\right]=\left[e_{\beta}, t_{i j}^{\alpha}\right]+\left[e_{\beta}, n_{i j}^{\alpha}\right] \operatorname{logo}(\beta)\left(t_{i j}^{\alpha}\right)=0$. Como $(\alpha+\beta+\gamma) \notin \Delta_{9}$ temos $0=\left[e_{\beta+\gamma}, x^{\alpha}\right]=\left[e_{\beta+\gamma}, t_{i j}^{\alpha}\right]+\left[e_{\beta+\gamma}, n_{i j}^{\alpha}\right] \operatorname{logo}(\beta+\gamma)\left(t_{i j}^{\alpha}\right)=0$. Portanto $x^{\alpha}=n_{i j}^{\alpha}$. Agora se,

$x^{\beta}=t_{i j}^{\beta}+n_{i j}^{\beta}$, então $\beta\left(t_{i j}^{\beta}\right)=0$ e, como $(\alpha+\beta) \notin \Delta_{9}$ então $0=\left[e_{\alpha}, x^{\beta}\right]=$ $\left[e_{\alpha}, t_{i j}^{\beta}\right]+\left[e_{\alpha}, n_{i j}^{\beta}\right] \operatorname{logo}(\alpha)\left(t_{i j}^{\beta}\right)=0$. Como $(\alpha+\beta+\gamma) \notin \Delta_{9}$ tem-se $0=$ $\left[e_{\alpha+\gamma}, x^{\beta}\right]=\left[e_{\alpha+\gamma}, t_{i j}^{\beta}\right]+\left[e_{\alpha+\gamma}, n_{i j}^{\beta}\right] \operatorname{logo}(\alpha+\gamma)\left(t_{i j}^{\beta}\right)=0$. Portanto $x^{\beta}=n_{i j}^{\beta}$. $x^{\gamma}=t_{i j}^{\gamma}+n_{i j}^{\gamma}$ então $\gamma\left(t_{i j}^{\gamma}\right)=0 \mathrm{e}, x^{\gamma}=\alpha\left(t_{i j}^{\gamma}\right) t_{1}+\beta\left(t_{i j}^{\gamma}\right) t_{2}+n_{i j}^{\gamma}$.

$x^{\alpha+\gamma}=t_{i j}^{\alpha+\gamma}+n_{i j}^{\alpha+\gamma}$ então $(\alpha+\gamma)\left(t_{i j}^{\alpha+\gamma}\right)=0$ e, $\beta\left(t_{i j}^{\alpha+\gamma}\right)=0$, como $(\alpha+\beta) \notin$ $\Delta_{9}$ então $0=\left[e_{\beta+\gamma}, x^{\alpha+\gamma}\right]=\left[e_{\beta+\gamma}, t_{i j}^{\alpha+\gamma}\right]+\left[e_{\beta+\gamma}, n_{i j}^{\alpha+\gamma}\right]$, logo $(\beta+\gamma)\left(t_{i j}^{\alpha+\gamma}\right)=0$. Portanto $x^{\alpha+\gamma}=n_{i j}^{\alpha+\gamma}$.

$x^{\beta+\gamma}=t_{i j}^{\beta+\gamma}+n_{i j}^{\beta+\gamma}$ então $(\beta+\gamma)\left(t_{i j}^{\beta+\gamma}\right)=0$ e $\alpha\left(t_{i j}^{\beta+\gamma}\right)=0$ e como $(\alpha+\beta) \notin$ $\Delta_{9}$ então $0=\left[e_{\alpha+\gamma}, x^{\beta+\gamma}\right]=\left[e_{\alpha+\gamma}, t_{i j}^{\beta+\gamma}\right]+\left[e_{\alpha+\gamma}, n_{i j}^{\beta+\gamma}\right]$, logo

$(\alpha+\gamma)\left(t_{i j}^{\beta+\gamma}\right)=0$. Portanto $x^{\beta+\gamma}=n_{i j}^{\beta+\gamma}$. Assim

$\left[L_{\alpha}, L_{\alpha}\right],\left[L_{\beta}, L_{\beta}\right],\left[L_{\alpha+\gamma}, L_{\alpha+\gamma}\right],\left[L_{\beta+\gamma}, L_{\beta+\gamma}\right] \subseteq N, \mathrm{e}$

$\left[L_{\gamma}, L_{\gamma}\right] \subseteq\left\langle t_{1}, t_{2}\right\rangle \oplus N$. Portanto $H=\sum_{\xi \in \Delta_{9}}\left[L_{\xi}, L_{\xi}\right] \subseteq\left\langle t_{1}, t_{2}\right\rangle \oplus N$, logo $\operatorname{dim}_{k}(T) \leq 2$. Uma contradição.

2.2.3.e. Quando $\Delta_{10}=\{\alpha, \beta, \gamma, \alpha+\gamma, \alpha+\beta+\gamma\}$, então

$$
\underline{L}=T \oplus N \oplus L_{\alpha} \oplus L_{\beta} \oplus L_{\gamma} \oplus L_{\alpha+\gamma} \oplus L_{\alpha+\beta+\gamma} .
$$

Se $x^{\alpha}=t_{i j}^{\alpha}+n_{i j}^{\alpha}$, então $\alpha\left(t_{i j}^{\alpha}\right)=0$ e $\beta\left(t_{i j}^{\alpha}\right)=0$. Também temos que, $0=$ $\left[e_{\alpha+\beta+\gamma}, x^{\alpha}\right]=\left[e_{\alpha+\beta+\gamma}, t_{i j}^{\alpha}\right]+\left[e_{\alpha+\beta+\gamma}, n_{i j}^{\alpha}\right] \operatorname{logo}(\alpha+\beta+\gamma)\left(t_{i j}^{\alpha}\right)=0$ e assim $\gamma\left(t_{i j}^{\alpha}\right)=0$. Portanto $x^{\alpha}=n_{i j}^{\alpha}$.

Se $x^{\beta}=t_{i j}^{\beta}+n_{i j}^{\beta}$, então $\beta\left(t_{i j}^{\beta}\right)=\gamma\left(t_{i j}^{\beta}\right)=\alpha\left(t_{i j}^{\beta}\right)=0$. Portanto $x^{\beta}=n_{i j}^{\beta}$ Se $x^{\gamma}=t_{i j}^{\gamma}+n_{i j}^{\gamma}$, então $\gamma\left(t_{i j}^{\gamma}\right)=0$ e $\beta\left(t_{i j}^{\gamma}\right)=0$. Também temos

$0=\left[e_{\alpha+\beta+\gamma}, x^{\gamma}\right]=\left[e_{\alpha+\beta+\gamma}, t_{i j}^{\gamma}\right]+\left[e_{\alpha+\beta+\gamma}, n_{i j}^{\gamma}\right] \operatorname{logo}(\alpha+\beta+\gamma)\left(t_{i j}^{\gamma}\right)=0 \mathrm{e}$ $\operatorname{assim} \alpha\left(t_{i j}^{\gamma}\right)=0$. Portanto $x^{\gamma}=n_{i j}^{\gamma}$. 
Se $x^{\alpha+\gamma}=t_{i j}^{\alpha+\gamma}+n_{i j}^{\alpha+\gamma}$ então, $(\alpha+\gamma)\left(t_{i j}^{\alpha}\right)=0$, portanto: $x^{\alpha+\gamma}=\alpha\left(t_{i j}^{\alpha+\gamma}\right) t_{1}+\beta\left(t_{i j}^{\alpha+\gamma}\right) t_{2}+\gamma\left(t_{i j}^{\alpha+\gamma}\right) t_{3}+n_{i j}^{\alpha+\gamma}$, com $\alpha\left(t_{i j}^{\alpha+\gamma}\right)=\gamma\left(t_{i j}^{\alpha+\gamma}\right)$. Se $x^{\alpha+\beta+\gamma}=t_{i j}^{\alpha+\beta+\gamma}+n_{i j}^{\alpha+\beta+\gamma}$ então $(\alpha+\beta+\gamma)\left(t_{i j}^{\alpha+\beta+\gamma}\right)=0$, e como $\alpha+\beta, \beta+\gamma \notin \Delta_{10}, \gamma\left(t_{i j}^{\alpha+\beta+\gamma}\right)=0, \alpha\left(t_{i j}^{\alpha+\beta+\gamma}\right)=0$ então $\beta\left(t_{i j}^{\alpha+\beta+\gamma}\right)=0$. Assim que: $\left[L_{\xi}, L_{\xi}\right] \subseteq N \forall \xi \in \Delta_{10} \backslash\{\alpha+\gamma\}$ e, $\left[L_{\alpha+\gamma}, L_{\alpha+\gamma}\right] \subseteq\left\langle t_{2}, t_{1}+t_{3}\right\rangle \oplus N$, então a $\operatorname{dim}_{k}(T) \leq 2$. Uma contradição. 2.2.3.f. Quando $\Delta_{11}=\{\alpha, \beta, \gamma, \beta+\gamma, \alpha+\beta+\gamma\}$, então

$$
\underline{L=T \oplus N \oplus L_{\alpha} \oplus L_{\beta} \oplus L_{\gamma} \oplus L_{\beta+\gamma} \oplus L_{\alpha+\beta+\gamma} .}
$$

Logo se $x^{\alpha}=t_{i j}^{\alpha}+n_{i j}^{\alpha}$ então $\alpha\left(t_{i j}^{\alpha}\right)=0$ e $\beta\left(t_{i j}^{\alpha}\right)=0=\gamma\left(t_{i j}^{\alpha}\right)$ portanto $x^{\alpha}=n_{i j}^{\alpha}$.

Se $x^{\beta}=t_{i j}^{\beta}+n_{i j}^{\beta}$ então $\alpha\left(t_{i j}^{\beta}\right)=0$ e $\alpha\left(t_{i j}^{\alpha}\right)=0$. Também temos que $0=$ $\left[e_{\alpha+\beta+\gamma}, x^{\beta}\right]=\left[e_{\alpha+\beta+\gamma}, t_{i j}^{\beta}\right]+\left[e_{\alpha+\beta+\gamma}, n_{i j}^{\beta}\right] \operatorname{logo}(\alpha+\beta+\gamma)\left(t_{i j}^{\beta}\right)=0$, então $\gamma\left(t_{i j}^{\beta}\right)=0$. Portanto, $x^{\beta}=n_{i j}^{\beta}$.

Se $x^{\gamma}=t_{i j}^{\gamma}+n_{i j}^{\gamma}$ então $\gamma\left(t_{i j}^{\gamma}\right)=0=\alpha\left(t_{i j}^{\gamma}\right)$. Por outro lado, $0=\left[e_{\alpha+\beta+\gamma}, x^{\gamma}\right]=$ $\left[e_{\alpha+\beta+\gamma}, t_{i j}^{\gamma}\right]+\left[e_{\alpha+\beta+\gamma}, n_{i j}^{\gamma}\right] \operatorname{logo}(\alpha+\beta+\gamma)\left(t_{i j}^{\gamma}\right)=0$ então $\beta\left(t_{i j}^{\gamma}\right)=0$. Logo $x^{\gamma}=t_{i j}^{\gamma}$.

Se $x^{\beta+\gamma}=t_{i j}^{\beta+\gamma}+n_{i j}^{\beta+\gamma}$ então $(\beta+\gamma)\left(t_{i j}^{\beta+\gamma}\right)=0$, assim $x^{\beta+\gamma}=\alpha\left(t_{i j}^{\beta+\gamma}\right) t_{1}+\beta\left(t_{i j}^{\beta+\gamma}\right) t_{2}+\gamma\left(t_{i j}^{\beta+\gamma}\right) t_{3}+n_{i j}^{\beta+\gamma} \operatorname{com} \beta\left(t_{i j}^{\beta+\gamma}\right)=\gamma\left(t_{i j}^{\beta+\gamma}\right)$ Se $x^{\alpha+\beta+\gamma}=t_{i j}^{\alpha+\beta+\gamma}+n_{i j}^{\alpha+\beta+\gamma}$ então $(\alpha+\beta+\gamma)\left(t_{i j}^{\alpha+\beta+\gamma}\right)=0$, e como $\alpha+\gamma$, $\alpha+\beta \notin \Delta_{11}$ então $\beta\left(t_{i j}^{\alpha+\beta+\gamma}\right)=\gamma\left(t_{i j}^{\alpha+\beta+\gamma}\right)=0$. Assim:

$\left[L_{\xi}, L_{\xi}\right] \subseteq N, \forall \xi \in \Delta_{11} \backslash\{\beta+\gamma\}$ e, $\left[L_{\beta+\gamma}, L_{\beta+\gamma}\right] \subseteq\left\langle t_{2}, t_{1}+t_{3}\right\rangle \oplus N$ $\operatorname{logo} H \subseteq\left\langle t_{2}, t_{1}+t_{3}\right\rangle \oplus N$, então a $\operatorname{dim}(T) \leq 2$. Um contradição.

- 2.2 .4 .

2.2.4.a. Se $\Delta_{12}=\{\alpha, \beta, \gamma, \alpha+\beta, \alpha+\gamma, \beta+\gamma\}$, então

$$
\underline{L=T \oplus N \oplus L_{\alpha} \oplus L_{\beta} \oplus L_{\gamma} \oplus L_{\alpha+\beta} \oplus L_{\alpha+\gamma} \oplus L_{\beta+\gamma} .}
$$

Se $x^{\alpha}=t^{\alpha}+n^{\alpha} \in\left[L_{\alpha}, L_{\alpha}\right]$ temos $\alpha\left(t^{\alpha}\right)=0$ e como $\alpha+\beta+\gamma \notin \Delta_{12}$, então: $\beta\left(t^{\alpha}\right)+\gamma\left(t^{\alpha}\right)=0$. Portanto $x^{\alpha}=\beta\left(t^{\alpha}\right) t_{2}+\gamma\left(t^{\alpha}\right) t_{3}+n^{\alpha} \operatorname{com} \beta\left(t^{\alpha}\right)=\gamma\left(t^{\alpha}\right)$. Se $x^{\beta}=t^{\beta}+n^{\beta} \in\left[L_{\beta}, L_{\beta}\right]$ temos $\beta\left(t^{\beta}\right)=0$ e como $(\alpha+\beta+\gamma) \notin \Delta_{12}$, então: $\alpha\left(t^{\beta}\right)+\gamma\left(t^{\beta}\right)=0$. Por tanto $x^{\beta}=\alpha\left(t^{\beta}\right) t_{1}+\gamma\left(t^{\beta}\right) t_{3}+n^{\beta}$, com $\alpha\left(t^{\beta}\right)=\gamma\left(t^{\beta}\right)$. Se $x^{\gamma}=t^{\gamma}+n^{\gamma} \in\left[L_{\gamma}, L_{\gamma}\right]$ temos $\gamma\left(t^{\gamma}\right)=0$ e como $\alpha+\beta+\gamma \notin \Delta_{12}$, então: $\alpha\left(t^{\gamma}\right)+\beta\left(t^{\gamma}\right)=0$. Por tanto $x^{\gamma}=\alpha\left(t^{\gamma}\right) t_{1}+\beta\left(t^{\gamma}\right) t_{2}+n^{\gamma}$, com $\alpha\left(t^{\gamma}\right)=\beta\left(t^{\gamma}\right)$. Se $x^{\alpha+\beta}=t^{\alpha+\beta}+n^{\alpha+\beta} \in\left[L_{\alpha+\beta}, L_{\alpha+\beta}\right]$ temos $(\alpha+\beta)\left(t^{\alpha+\beta}\right)=0$ e como $(\alpha+\beta+\gamma) \notin \Delta_{12}$, então $\gamma\left(t^{\alpha+\beta}\right)=0$. Portanto $x^{\alpha+\beta}=\alpha\left(t^{\alpha+\beta}\right) t_{1}+\beta\left(t^{\alpha+\beta}\right) t_{2}+n^{\alpha+\beta} \operatorname{com} \alpha\left(t^{\alpha+\beta}\right)=\beta\left(t^{\alpha+\beta}\right)$.

Se $x^{\alpha+\gamma}=t^{\alpha+\gamma}+n^{\alpha+\gamma} \in\left[L_{\alpha+\gamma}, L_{\alpha+\gamma}\right]$ temos $(\alpha+\gamma)\left(t^{\alpha+\gamma}\right)=0$ e como $(\alpha+\beta+\gamma) \notin \Delta_{12}$, então $\beta\left(t^{\alpha+\gamma}\right)=0$. Portanto 
$x^{\alpha+\gamma}=\alpha\left(t^{\alpha+\gamma}\right) t_{1}+\beta\left(t^{\alpha+\gamma}\right) t_{2}+n^{\alpha+\gamma}, \operatorname{com} \alpha\left(t^{\alpha+\gamma}\right)=\beta\left(t^{\alpha+\gamma}\right)$.

Se $x^{\beta+\gamma}=t^{\beta+\gamma}+n^{\beta+\gamma} \in\left[L_{\beta+\gamma}, L_{\beta+\gamma}\right]$ temos $(\beta+\gamma)\left(t^{\beta+\gamma}\right)=0$ e como $(\alpha+\beta+\gamma) \notin \Delta_{12}$, então $\alpha\left(t^{\beta+\gamma}\right)=0$. Portanto

$x^{\beta+\gamma}=\beta\left(t^{\beta+\gamma}\right) t_{2}+\gamma\left(t^{\beta+\gamma}\right) t_{3}+n^{\beta+\gamma} \operatorname{com} \gamma\left(t^{\alpha+\gamma}\right)=\beta\left(t^{\alpha+\gamma}\right)$.

Assim que:

$\left[L_{\alpha}, L_{\alpha}\right],\left[L_{\beta+\gamma}, L_{\beta+\gamma}\right] \subseteq\left\langle t_{2}+t_{3}\right\rangle \oplus N$.

$\left[L_{\beta}, L_{\beta}\right],\left[L_{\alpha+\gamma}, L_{\alpha+\gamma}\right] \subseteq\left\langle t_{1}+t_{3}\right\rangle \oplus N$.

$\left[L_{\gamma}, L_{\gamma}\right],\left[L_{\alpha+\beta}, L_{\alpha+\beta}\right] \subseteq\left\langle t_{1}+t_{3}\right\rangle \oplus N$.

Afirmação. $t_{1} \notin[L, L]$.

$\overline{\text { Ja que, se }} t_{1} \in[L, L] \subseteq \sum_{\xi \in \Delta_{12}}\left[L_{\xi}, L_{\xi}\right] \subseteq T_{0} \oplus N$

onde $T_{0}:=k\left\{t_{2}+t_{3}\right\} \oplus k\left\{t_{1}+t_{2}\right\} \oplus k\left\{t_{1}+t_{3}\right\}$ então

$t_{1}=\delta_{1}\left(t_{2}+t_{3}\right)+\delta_{2}\left(t_{1}+t_{2}\right)+\delta_{3}\left(t_{1}+t_{3}\right)+n$ para algum $\delta_{i} \in k$ e $n \in N$,

$\operatorname{logo}\left(1+\delta_{2}+\delta_{3}\right) t_{1}+\left(\delta_{1}+\delta_{2}\right) t_{2}+\left(\delta_{1}+\delta_{3}\right) t_{3}=n$. Como $T \cap N=0$, então $\left(1+\delta_{2}+\delta_{3}\right) t_{1}+\left(\delta_{1}+\delta_{2}\right) t_{2}+\left(\delta_{1}+\delta_{3}\right) t_{3}=n=0$.

Como $t_{1}, t_{2}, t_{3}$ são linearmente independente, então temos o seguinte sistema de equações

$$
\begin{cases}1+\delta_{2}+\delta_{3} & =0 \\ \delta_{1}+\delta_{2} & =0 \\ \delta_{1}+\delta_{3} & =0\end{cases}
$$

que não tem solução em $k$, o qual é uma contradição. Logo a afirmação contradiz o fato de $T \subseteq[L, L]$.

2.2.4.b. Quando $\Delta_{13}=\{\alpha, \beta, \gamma, \alpha+\beta, \alpha+\gamma, \alpha+\beta+\gamma\}$, então

$$
\underline{L=T \oplus N \oplus L_{\alpha} \oplus L_{\beta} \oplus L_{\gamma} \oplus L_{\alpha+\beta} \oplus L_{\alpha+\gamma} \oplus L_{\alpha+\beta+\gamma}} .
$$

Temos que $\left[L_{\alpha}, L_{\alpha}\right],\left[L_{\alpha+\beta+\gamma}, L_{\alpha+\beta+\gamma}\right] \subseteq\left\langle t_{2}+t_{3}\right\rangle \oplus N$. $\left[L_{\beta}, L_{\beta}\right],\left[L_{\gamma}, L_{\gamma}\right] \subseteq\left\langle t_{1}\right\rangle \oplus N$.

$\left[L_{\alpha+\beta}, L_{\alpha+\beta}\right],\left[L_{\alpha+\gamma}, L_{\alpha+\gamma}\right] \subseteq\left\langle t_{1}+t_{2}+t_{3}\right\rangle \oplus N$.

Pelo mesmo argumento do caso 2.2.4.a obtemos que $t_{2} \notin[L, L]$.

Uma contradição, já que $T \subseteq[L, L]$.

2.2.4.c. Se $\Delta_{14}=\{\alpha, \beta, \gamma, \alpha+\beta, \beta+\gamma, \alpha+\beta+\gamma\}$, temos

$$
\underline{L=T \oplus N \oplus L_{\alpha} \oplus L_{\beta} \oplus L_{\gamma} \oplus L_{\alpha+\beta} \oplus L_{\beta+\gamma} \oplus L_{\alpha+\beta+\gamma}} .
$$

Se $x^{\alpha}=t^{\alpha}+n^{\alpha} \in\left[L_{\alpha}, L_{\alpha}\right]$ então $\alpha\left(t^{\alpha}\right)=0$ e como $(\alpha+\gamma) \notin \Delta_{14}$ então $\gamma\left(t^{\alpha}\right)=0$ então $\left[L_{\alpha}, L_{\alpha}\right] \subseteq\left\langle t_{2}\right\rangle \oplus N$.

Se $x^{\beta}=t^{\beta}+n^{\beta} \in\left[L_{\beta}, L_{\beta}\right]$ então $\beta\left(t^{\beta}\right)=0.0=\left[e_{\alpha+\beta+\gamma}, x^{\beta}\right]=\left[e_{\alpha+\beta+\gamma}, t^{\beta}\right]+$ $\left[e_{\alpha+\beta+\gamma}, n^{\beta}\right]=(\alpha+\beta+\gamma)\left(t^{\beta}\right) e_{\alpha+\beta+\gamma}+\left[e_{\alpha+\beta+\gamma}, n^{\beta}\right]$ então $(\alpha+\beta+\gamma)\left(t^{\beta}\right)=0$ então $(\alpha+\gamma)\left(t^{\beta}\right)=0, \operatorname{logo} x^{\beta}=\alpha\left(t^{\beta}\right) t_{1}+\gamma\left(t^{\beta}\right) t_{3}+n^{\beta} \operatorname{com} \alpha\left(t^{\beta}\right)=\gamma\left(t^{\beta}\right)$. Portanto $\left[L_{\beta}, L_{\beta}\right] \subseteq\left\langle t_{1}+t_{3}\right\rangle \oplus N$.

Se $x^{\gamma}=t^{\gamma}+n^{\gamma} \in\left[L_{\gamma}, L_{\gamma}\right]$ então $\gamma\left(t^{\gamma}\right)=0 . \quad 0=\left[e_{\alpha}, x^{\gamma}\right]=\left[e_{\alpha}, t^{\gamma}\right]=$ $\alpha\left(t^{\gamma}\right)+\left[e_{\alpha}, n^{\gamma}\right]$, então $\alpha\left(t^{\gamma}\right)=0$, assim

$x^{\gamma}=\beta\left(t^{\gamma}\right) t_{2}+n^{\gamma}$. Portanto, $\left[L_{\gamma}, L_{\gamma}\right] \subseteq\left\langle t_{2}\right\rangle \oplus N$. 
Se $x^{\alpha+\beta}=t^{\alpha+\beta}+n^{\alpha+\beta} \in\left[L_{\alpha+\beta}, L_{\alpha+\beta}\right]$ então $(\alpha+\beta)\left(t^{\alpha+\beta}\right)=0 . \quad 0=$ $\left[e_{\beta+\gamma}, x^{\alpha+\beta}\right]=\left[e_{\beta+\gamma}, t^{\alpha+\beta}\right]+\left[e_{\beta+\gamma}, n^{\alpha+\beta}\right]=(\beta+\gamma)\left(t^{\alpha+\beta}\right) e_{\beta+\gamma}+\left[e_{\beta+\gamma}, n^{\alpha+\beta}\right]$ então $(\beta+\gamma)\left(t^{\alpha+\beta}\right)=0, \operatorname{logo} x^{\alpha+\beta}=\alpha\left(t^{\alpha+\beta}\right) t_{1}+\beta\left(t^{\alpha+\beta}\right) t_{2}+\gamma\left(t^{\alpha+\beta}\right) t_{3}+$ $n^{\alpha+\beta} \operatorname{com} \alpha\left(t^{\alpha+\beta}\right)=\beta\left(t^{\alpha+\beta}\right)=\gamma\left(t^{\alpha+\beta}\right)$.

Portanto, $\left[L_{\alpha+\beta}, L_{\alpha+\beta}\right] \subseteq\left\langle t_{1}+t_{2}+t_{3}\right\rangle \oplus N$.

Se $x^{\beta+\gamma}=t^{\beta+\gamma}+n^{\beta+\gamma} \in\left[L_{\beta+\gamma}, L_{\beta+\gamma}\right]$ então $(\beta+\gamma)\left(t^{\beta+\gamma}\right)=0 . \quad 0=\left[e_{\alpha+\beta}, x^{\beta+\gamma}\right]=\left[e_{\alpha+\beta}, t^{\beta+\gamma}\right]+\left[e_{\alpha+\beta}, n^{\beta+\gamma}\right]=$ $(\alpha+\beta)\left(t^{\beta+\gamma}\right) e_{\alpha+\beta}+\left[e_{\alpha+\beta}, n^{\beta+\gamma}\right]$ então $(\alpha+\beta)\left(t^{\beta+\gamma}\right)=0, \operatorname{logo} x^{\beta+\gamma}=$ $\alpha\left(t^{\beta+\gamma}\right) t_{1}+\beta\left(t^{\beta+\gamma}\right) t_{2}+\gamma\left(t^{\beta+\gamma}\right) t_{3}+n^{\beta+\gamma} \operatorname{com} \alpha\left(t^{\beta+\gamma}\right)=\beta\left(t^{\beta+\gamma}\right)=\gamma\left(t^{\beta+\gamma}\right)$. Portanto $\left[L_{\beta+\gamma}, L_{\beta+\gamma}\right] \subseteq\left\langle t_{1}+t_{2}+t_{3}\right\rangle \oplus N$

Se $x^{\alpha+\beta+\gamma}=t^{\alpha+\beta+\gamma}+n^{\alpha+\beta+\gamma} \in\left[L_{\alpha+\beta+\gamma}, L_{\alpha+\beta+\gamma}\right]$ então $(\alpha+\beta+\gamma)\left(t^{\alpha+\beta+\gamma}\right)=$ 0 . $0=\left[e_{\beta}, x^{\alpha+\beta+\gamma}\right]=\left[e_{\beta}, t^{\alpha+\beta+\gamma}\right]+\left[e_{\beta}, n^{\alpha+\beta+\gamma}\right]=\beta\left(t^{\alpha+\beta+\gamma}\right) e_{\beta}+\left[e_{\beta}, n^{\alpha+\beta+\gamma}\right]$ então $\beta\left(t^{\alpha+\beta+\gamma}\right)=0, \operatorname{logo} x^{\alpha+\beta+\gamma}=\alpha\left(t^{\alpha+\beta+\gamma}\right) t_{1}+\beta\left(t^{\alpha+\beta+\gamma}\right) t_{2}+\gamma\left(t^{\alpha+\beta+\gamma}\right) t_{3}+$ $n^{\alpha+\beta+\gamma} \operatorname{com} \alpha\left(t^{\alpha+\beta+\gamma}\right)=\gamma\left(t^{\alpha+\beta+\gamma}\right)$.

Portanto $\left[L_{\alpha+\beta+\gamma}, L_{\alpha+\beta+\gamma}\right] \subseteq\left\langle t_{1}+t_{3}\right\rangle \oplus N$.

Afirmação. $t_{1} \notin[L, L]$.

Já que, se $t_{1} \in[L, L] \subseteq \sum_{\xi \in \Delta_{14}}\left[L_{\xi}, L_{\xi}\right] \subseteq T_{0} \oplus N$

onde $T_{0}:=k\left\{t_{2}\right\} \oplus k\left\{t_{1}+t_{3}\right\} \oplus k\left\{t_{1}+t_{2}+t_{3}\right\}$ então

$t_{1}=\delta_{1}\left(t_{2}\right)+\delta_{2}\left(t_{1}+t_{3}\right)+\delta_{3}\left(t_{1}+t_{2}+t_{3}\right)+n$ para algum $\delta_{i} \in k$ e $n \in N$, $\operatorname{logo}\left(1+\delta_{2}+\delta_{3}\right) t_{1}+\left(\delta_{1}+\delta_{2}\right) t_{2}+\left(\delta_{1}+\delta_{3}\right) t_{3}=n$. Como $T \cap N=0$, então $\left(1+\delta_{2}+\delta_{3}\right) t_{1}+\left(\delta_{1}+\delta_{2}\right) t_{2}+\left(\delta_{1}+\delta_{3}\right) t_{3}=n=0$.

Como $t_{1}, t_{2}, t_{3}$ são linearmente independente, então temos o sistema

$$
\begin{cases}1+\delta_{2}+\delta_{3} & =0 \\ \delta_{1}+\delta_{2} & =0 \\ \delta_{1}+\delta_{3} & =0\end{cases}
$$

que não tem solucão, o qual é uma contradição. Logo a afirmação contradiz o fato $T \subseteq[L, L]$.

2.2.4.d. Quando $\Delta_{15}=\{\alpha, \beta, \gamma, \alpha+\gamma, \beta+\gamma, \alpha+\beta+\gamma\}$, então

$$
\underline{L=T \oplus N \oplus L_{\alpha} \oplus L_{\beta} \oplus L_{\gamma} \oplus L_{\alpha+\gamma} \oplus L_{\beta+\gamma} \oplus L_{\alpha+\beta+\gamma}}
$$

Temos que $(\alpha+\beta) \notin \Delta_{15}$. Assim:

Se $x^{\alpha}=t^{\alpha}+n^{\alpha} \in\left[L_{\alpha}, L_{\alpha}\right]$, então $\alpha\left(t^{\alpha}\right)=0$ e como $(\alpha+\beta) \notin \Delta_{15}$, então $\beta\left(t^{\alpha}\right)=0$, então $\left[L_{\alpha}, L_{\alpha}\right] \subseteq\left\langle t_{3}\right\rangle \oplus N$.

Se $x^{\beta}=t^{\beta}+n^{\beta} \in\left[L_{\beta}, L_{\beta}\right]$, então $\beta\left(t^{\beta}\right)=0$ e como $(\alpha+\beta) \notin \Delta_{15}$, então $\alpha\left(t^{\beta}\right)=0$ então $\left[L_{\beta}, L_{\beta}\right] \subseteq\left\langle t_{3}\right\rangle \oplus N$.

Se $x^{\gamma}=t^{\gamma}+n^{\gamma} \in\left[L_{\gamma}, L_{\gamma}\right]$, então $\gamma\left(t^{\gamma}\right)=0$ e como $0=\left[e_{\alpha+\beta+\gamma}, x^{\gamma}\right]=$ $\left[e_{\alpha+\beta+\gamma}, t^{\gamma}\right]+\left[e_{\alpha+\beta+\gamma}, n^{\gamma}\right]=(\alpha+\beta+\gamma)\left(t^{\gamma}\right) e_{\alpha+\beta+\gamma}+\left[e_{\alpha+\beta+\gamma}, n^{\gamma}\right]$ então $(\alpha+\beta+\gamma)\left(t^{\gamma}\right)=0, \operatorname{logo}(\alpha+\beta)\left(t^{\gamma}\right)=0$. Tem-se

$x^{\gamma}=\alpha\left(t^{\gamma}\right) t_{1}+\beta\left(t^{\gamma}\right) t_{2}+n^{\gamma} \operatorname{com} \alpha\left(t^{\gamma}\right)=\beta\left(t^{\gamma}\right)$ e assim

$\left[L_{\gamma}, L_{\gamma}\right] \subseteq\left\langle t_{1}+t_{2}\right\rangle \oplus N$. 
Se $x^{\alpha+\gamma}=t^{\alpha+\gamma}+n^{\alpha+\gamma} \in\left[L_{\alpha+\gamma}, L_{\alpha+\gamma}\right]$, então $(\alpha+\gamma)\left(t^{\alpha+\gamma}\right)=0$ e como $0=\left[e_{\beta+\gamma}, x^{\alpha+\gamma}\right]=\left[e_{\beta+\gamma}, t^{\alpha+\gamma}\right]+\left[e_{\beta+\gamma}, n^{\alpha+\gamma}\right]=$ $(\beta+\gamma)\left(t^{\alpha+\gamma}\right) e_{\beta+\gamma}+\left[e_{\beta+\gamma}, n^{\alpha+\gamma}\right]$ então $(\beta+\gamma)\left(t^{\alpha+\gamma}\right)=0$, logo tem-se $x^{\alpha+\gamma}=\alpha\left(t^{\alpha+\gamma}\right) t_{1}+\beta\left(t^{\alpha+\gamma}\right) t_{2}+\gamma\left(t^{\alpha+\gamma}\right) t_{3}+n^{\alpha+\gamma}, \operatorname{com} \alpha\left(t^{\alpha+\gamma}\right)=\beta\left(t^{\alpha+\gamma}\right)=$ $\gamma\left(t^{\alpha+\gamma}\right)$ e assim $\left[L_{\alpha+\gamma}, L_{\alpha+\gamma}\right] \subseteq\left\langle t_{1}+t_{2}+t_{3}\right\rangle \oplus N$.

Se $x^{\beta+\gamma}=t^{\beta+\gamma}+n^{\beta+\gamma} \in\left[L_{\beta+\gamma}, L_{\beta+\gamma}\right]$ então $(\beta+\gamma)\left(t^{\beta+\gamma}\right)=0$ e como $0=\left[e_{\alpha+\gamma}, x^{\beta+\gamma}\right]=\left[e_{\alpha+\gamma}, t^{\beta+\gamma}\right]+\left[e_{\alpha+\gamma}, n^{\beta+\gamma}\right]=(\alpha+\gamma)\left(t^{\beta+\gamma}\right) e_{\alpha+\gamma}+\left[e_{\alpha+\gamma}, n^{\beta+\gamma}\right]$ então $(\alpha+\gamma)\left(t^{\beta+\gamma}\right)=0$, logo tem-se $x^{\beta+\gamma}=\alpha\left(t^{\beta+\gamma}\right) t_{1}+\beta\left(t^{\beta+\gamma}\right) t_{2}+\gamma\left(t^{\beta+\gamma}\right) t_{3}+n^{\beta+\gamma}, \mathrm{com}$ $\alpha\left(t^{\beta+\gamma}\right)=\beta\left(t^{\beta+\gamma}\right)=\gamma\left(t^{\beta+\gamma}\right)$ e assim $\left[L_{\beta+\gamma}, L_{\beta+\gamma}\right] \subseteq\left\langle t_{1}+t_{2}+t_{3}\right\rangle \oplus N$.

Se $x^{\alpha+\beta+\gamma}=t^{\alpha+\beta+\gamma}+n^{\alpha+\beta+\gamma} \in\left[L_{\alpha+\beta+\gamma}, L_{\alpha+\beta+\gamma}\right]$ então $(\alpha+\beta+\gamma)\left(t^{\alpha+\beta+\gamma}\right)=$ 0 e como $0=\left[e_{\gamma}, x^{\alpha+\beta+\gamma}\right]=\left[e_{\gamma}, t^{\alpha+\beta+\gamma}\right]+\left[e_{\gamma}, n^{\alpha+\beta+\gamma}\right]=\gamma\left(t^{\alpha+\beta+\gamma}\right) e_{\gamma}+$ $\left[e_{\gamma}, n^{\alpha+\beta+\gamma}\right]$ então $\gamma\left(t^{\alpha+\beta+\gamma}\right)=0$, logo tem-se $x^{\alpha+\beta+\gamma}=\alpha\left(t^{\alpha+\beta+\gamma}\right) t_{1}+\beta\left(t^{\alpha+\beta+\gamma}\right) t_{2}+\gamma\left(t^{\alpha+\beta+\gamma}\right) t_{3}+n^{\alpha+\beta+\gamma}, \mathrm{com}$ $\alpha\left(t^{\alpha+\beta+\gamma}\right)=\beta\left(t^{\alpha+\beta+\gamma}\right)$ e assim $\left[L_{\alpha+\beta+\gamma}, L_{\alpha+\beta+\gamma}\right] \subseteq\left\langle t_{1}+t_{2}\right\rangle \oplus N$.

Seja $T_{0}=k\left\{t_{3}\right\} \oplus k\left\{t_{1}+t_{2}\right\} \oplus k\left\{t_{1}+t_{2}+t_{3}\right\}$, $\operatorname{logo} H \subseteq[L, L] \subseteq \sum_{\xi \in \Delta_{15}}\left[L_{\xi}, L_{\xi}\right] \subseteq T_{0} \oplus N$. Se $t_{1} \in[L, L]$ então existem $\delta_{1}, \delta_{2}, \delta_{3}$ em $k$ tal que $t_{1}=\delta_{1} t_{3}+\delta_{2}\left(t_{1}+t_{2}\right)+\delta_{3}\left(t_{1}+t_{2}+t_{3}\right)$ assim temos o sistema de equações

$$
\begin{cases}1+\delta_{2}+\delta_{3} & =0 \\ \delta_{2}+\delta_{3} & =0 \\ \delta_{1}+\delta_{3} & =0\end{cases}
$$

Resolvendo o sistema, temos que $0 \delta_{2}+0 \delta_{3}=1$, o que não pode ocorrer e $\operatorname{assim} t_{1} \notin[L, L]$, mas $T \subseteq[L, L]$. Uma contradição.

\subsection{Análise de $L=H \oplus \sum_{\xi \in \Delta} \oplus L_{\xi}$, com $\Delta=T^{*} \backslash\{0\}$.}

Na seção 2.3, se demonstrou que quando o $\operatorname{Card}(\Delta)<7$, não existem 2-álgebras de Lie simples de dimensão finita de posto toral 3, com decomposição de Cartan $L^{\Delta}$. Nesta secão, vamos estudar a decomposição de $\operatorname{Cartan} L^{\Delta}$, quando $\operatorname{card}(\Delta)=7$, isto é, quando $\Delta=\Delta_{0}:=T^{*} \backslash\{0\}$.

Suponhamos que $(L,[2])$ é uma 2-álgebra de Lie simples, de posto toral 3, de dimensão maior ou igual a 10, cuja decomposição de Cartan respeito a $T$ é:

$$
\underline{L}=T \oplus N \oplus L_{\alpha} \oplus L_{\beta} \oplus L_{\gamma} \oplus L_{\alpha+\beta} \oplus L_{\alpha+\gamma} \oplus L_{\beta+\gamma} \oplus L_{\alpha+\beta+\gamma} .
$$

Antes de começar a estudar estas álgebras, é conveniente que se faça o seguinte cometário sobre notação.

Observação 2.8. A 10-tupla

$\left(\operatorname{dim}_{k}(L): \operatorname{dim}_{k}(T), \operatorname{dim}_{k}(N), \operatorname{dim}_{k}\left(L_{\alpha}\right), \operatorname{dim}_{k}\left(L_{\beta}\right), \operatorname{dim}_{k}\left(L_{\gamma}\right), \operatorname{dim}_{k}\left(L_{\alpha+\beta}\right), \operatorname{dim}_{k}\left(L_{\alpha+\gamma}\right), \operatorname{dim}_{k}\left(L_{\beta+\gamma}\right), \operatorname{dim}_{k}\left(L_{\alpha+\beta+\gamma}\right)\right)$ 
se refere ao 2-álgebras de Lie simples $(L,[2])$ de posto toral 3 com decomposição de Cartan $L=T \oplus N \oplus \sum_{\xi \in \Delta} \oplus L_{\xi}$ com seu respectiva dimensão e, as dimensões de seus espaços raizes.

Lembre que nós estamos considerando : $\operatorname{dim}_{k}\left(L_{\alpha}\right) \geq \operatorname{dim}_{k}\left(L_{\beta}\right) \geq \operatorname{dim}_{k}\left(L_{\gamma}\right) \geq \operatorname{dim}_{k}\left(L_{\xi}\right)>0$ para $\xi \notin \Delta \backslash\{\alpha, \beta, \alpha+\beta\}$.

Agora fixando a dimensão de $(L,[2])$, estudaremos seu estrutura quando $\Delta=T^{*} \backslash\{0\}$.

- 2.3.1. Se $\operatorname{dim}_{k}(L)=10$, temos $(10: 3,0,1,1,1,1,1,1,1)$, nesse caso $I:=\sum_{\xi \in \Delta} \oplus L_{\xi}$ é um ideal de $L$, portanto $L$ não é simples. Uma contradição.

- 2.3.2. Se $\operatorname{dim}_{k}(L)=11$, temos os seguintes casos:

2.3.2.a. (11:3, $0,2,1,1,1,1,1,1)$. Nesse caso, como

$T \subseteq \sum_{\xi \in \Delta}\left[L_{\xi}, L_{\xi}\right] \subseteq\left[L_{\alpha}, L_{\alpha}\right]$ então a $\operatorname{dim}_{k}(T) \leq 1$. Uma contradição.

2.3.2.b. $(11: 3,1,1,1,1,1,1,1,1)$. Então $I:=N \oplus \sum_{\xi \in \Delta} \oplus L_{\xi}$ é um ideal de $L$. Uma contradição.

- 2.3.3. Se $\operatorname{dim}_{k}(L)=12$. Temos os seguintes casos:

2.3.3.a. (12:3, $0,3,1,1,1,1,1,1)$. Seja $e_{\alpha} \in\left[L_{\alpha}, L_{\alpha}\right]$, então existem $\delta_{1}, \delta_{2}, \delta_{3} \in k$ tal que $e_{\alpha}=\delta_{1} t_{1}+\delta_{2} t_{2}+\delta_{3} t_{3}, \operatorname{logo} 0=\left[e_{\alpha}, e_{\alpha}\right]=\delta_{1} \alpha\left(t_{1}\right) e_{\alpha}+\delta_{2} \alpha\left(t_{2}\right) e_{\alpha}+$ $\delta_{3} \alpha\left(t_{3}\right) e_{\alpha}=\delta_{1} e_{\alpha}$, então $\delta_{1}=0, \operatorname{logo} e_{\alpha}=\delta_{2} t_{2}+\delta_{3} t_{3}$, isto é, $e_{\alpha} \in\left\langle t_{2}, t_{3}\right\rangle$, $\operatorname{assim}\left[L_{\alpha}, L_{\alpha}\right] \subseteq\left\langle t_{2}, t_{3}\right\rangle$. Como $T \subseteq \sum_{\xi \in \Delta}\left[L_{\xi}, L_{\xi}\right] \subseteq\left[L_{\alpha}, L_{\alpha}\right] \subseteq\left\langle t_{2}, t_{3}\right\rangle$. Então a $\operatorname{dim}_{k}(T) \leq 2$. Uma contradição.

2.3.3.b. $(12: 3,0,2,2,1,1,1,1,1)$. Então $T \subseteq \sum_{\xi \in \Delta}\left[L_{\xi}, L_{\xi}\right] \subseteq\left[L_{\alpha}, L_{\alpha}\right]+\left[L_{\beta}, L_{\beta}\right]$ $\operatorname{logo}_{\operatorname{dim}_{k}}(T) \leq \operatorname{dim}_{k}\left(\left[L_{\alpha}, L_{\alpha}\right]\right)+\operatorname{dim}_{k}\left(\left[L_{\beta}, L_{\beta}\right]\right) \leq 1+1=2$.

2.3.3.c. $(12: 3,1,2,1,1,1,1,1,1)$.

$H \subseteq \sum_{\xi \in \Delta}\left[L_{\xi}, L_{\xi}\right]=\left[L_{\alpha}, L_{\alpha}\right]$ e como $\operatorname{dim}_{k}\left(L_{\alpha}\right)=2$, então

$\operatorname{dim}_{k}\left(\left[L_{\alpha}, L_{\alpha}\right]\right) \leq 1$. Assim que, $\operatorname{dim}_{k}(H) \leq 1$. Uma contradição.

2.3.3.d. (12:3, 2, 1, 1, 1, 1, 1, 1, 1). Temos que $I:=N \oplus \sum_{\xi \in \Delta} \oplus L_{\xi}$ é um ideal de $L$. Uma contradição.

- 2.3.4. Se $\operatorname{dim}_{k}(L)=13$, então temos que:

2.3.4.a. $(13: 3,0,4,1,1,1,1,1,1)$.

2.3.4.b. $(13: 3,0,3,2,1,1,1,1,1)$.

2.3.4.c. $(13: 3,0,2,2,2,1,1,1,1)$.

Para estos três casos temos o seguinte:

Seja $e_{\beta} \in L_{\beta}$ elemento da base de $L_{\beta}$. Logo $e_{\beta}^{[2]}:=t_{\beta} \in T$ então existe $\delta_{1}, \delta_{2}, \delta_{3} \in k$ tal que $e_{\beta}^{[2]}=\delta_{1} t_{1}+\delta_{2} t_{2}+\delta_{3} t_{3}$, como $\beta\left(e_{\beta}^{[2]}\right)=0$, então $\delta_{2}=0 . \operatorname{Logo} t_{\beta}=\delta_{1} t_{1}+\delta_{3} t_{3}$ para algum $\delta_{1}, \delta_{3} \in k$, assim $L_{\beta}^{[2]} \subseteq\left\langle t_{1}, t_{3}\right\rangle$. Se escolhemos $\delta_{1} \neq 0 \operatorname{logo} \alpha\left(t_{\beta}\right)=\delta_{1} \neq 0$, pela proposição 2.4 temos que $L_{\alpha} \simeq L_{\alpha+\beta}$, mas isto é impossivel, já que $\operatorname{dim}_{k}\left(L_{\alpha}\right) \neq \operatorname{dim}_{k}\left(L_{\alpha+\beta}\right)=1$. 
2.3.4.d. (13:3,1,1,1,1,1,1,1). Então $I:=N \oplus \sum_{\xi \in \Delta} \oplus L_{\xi}$ é um ideal de $L$. Uma contradição.

2.3.4.e. $(13: 3,2,2,1,1,1,1,1,1)$. Temos $H \subseteq \sum_{\xi \in \Delta}\left[L_{\xi}, L_{\xi}\right]=\left[L_{\alpha}, L_{\alpha}\right]$ então $\operatorname{dim}_{k}(H) \leq 1$. Uma contradição.

2.3.4.f. $(13: 3,1,3,1,1,1,1,1,1)$. Temos $H \subseteq \sum_{\xi \in \Delta}\left[L_{\xi}, L_{\xi}\right]=$ $=\left[L_{\alpha}, L_{\alpha}\right] \subseteq\left\langle t_{2}, t_{3}\right\rangle, \operatorname{dim}_{k}(H) \leq 2$. Uma contradição.

2.3.4.g. (13:3,1,2,2,1,1,1,1,1). Temos $H \subseteq \sum_{\xi \in \Delta}\left[L_{\xi}, L_{\xi}\right]=$ $=\left[L_{\alpha}, L_{\alpha}\right]+\left[L_{\beta}, L_{\beta}\right]$, assim que $\operatorname{dim}_{k}(H) \leq 2$. Uma contradição.

- 2.3.5. Se $\operatorname{dim}_{k}(L)=14$. Então temos os seguintes casos:
2.3.5.a. $(14: 3,0,5,1,1,1,1,1,1)$
2.3.5.g. $(14: 3,1,3,2,1,1,1,1,1)$
2.3.5.b. $(14: 3,0,4,2,1,1,1,1,1)$
2.3.5.h. $(14: 3,1,4,1,1,1,1,1,1)$
2.3.5.c. $(14: 3,0,3,3,1,1,1,1,1)$
2.3.5.i. $(14: 3,2,2,2,1,1,1,1,1)$
2.3.5.d. $(14: 3,0,3,2,2,1,1,1,1)$
2.3.5.j. $(14: 3,2,3,1,1,1,1,1,1)$
2.3.5.e. $(14: 3,0,2,2,2,2,1,1,1)$
2.3.5.k. $(14: 3,3,2,1,1,1,1,1,1)$
2.3.5.f. $(14: 3,1,2,2,2,1,1,1,1)$
2.3.5.1. $(14: 3,4,1,1,1,1,1,1,1)$

Seja $e_{\beta}$ um elemento da base de $L_{\beta}$. Como $e_{\beta}^{[2]} \in L_{\beta}^{[2]} \subseteq H=T \oplus N$ então existem $t_{\beta} \in T$ e $n_{\beta} \in N$ tal que $e_{\beta}^{[2]}=t_{\beta}+n_{\beta}$. Como $t_{\beta} \in T$ existem $\delta_{1}, \delta_{2}, \delta_{3}$ em $k$, tal que $t_{\beta}=\delta_{1} t_{1}+\delta_{2} t_{2}+\delta_{3} t_{3}$. Por outro lado, $0=\left[e_{\beta}, e_{\beta}^{[2]}\right]=\beta\left(t_{\beta}\right) e_{\beta}+\left[e_{\beta}, n_{\beta}\right] \operatorname{logo}$ $\operatorname{ad}\left(n_{\beta}\right)\left(e_{\beta}\right)=\beta\left(t_{\beta}\right) e_{\beta}$, como $n_{\beta}$ é nilpotente então $\operatorname{ad}\left(n_{\beta}\right)$ é nilpotente, portanto $\beta\left(t_{\beta}\right)=\delta_{2}=0$. Assim que, $e_{\beta}^{[2]}=\delta_{1} t_{1}+\delta_{3} t_{3}+n_{\beta}$. Escolhendo $\delta_{1} \neq 0$ temos que $t_{\beta} \neq 0$ e $\alpha\left(t_{\beta}\right)=\delta_{1} \neq 0$, logo pela proposição $2.4, L_{\alpha} \simeq L_{\alpha+\beta}$. Portanto os casos anteriores, exceto os casos 2.3.5.e e 2.3.5.l, são impossivel já que

$\operatorname{dim}_{k}\left(L_{\alpha}\right) \neq \operatorname{dim}_{k}\left(L_{\alpha+\beta}\right)$. Para o caso 2.3.5.e, seja $e_{\gamma}$ um elemento da base de $L_{\gamma}$. Como $e_{\gamma}^{[2]} \in L_{\gamma}^{[2]} \subseteq H=T \oplus N$, então existem $t_{\gamma} \in T$ e $n_{\gamma} \in N$ tal que $e_{\gamma}^{[2]}=t_{\gamma}+n_{\gamma}$. Como $t_{\gamma} \in T$ existem $\delta_{1}, \delta_{2}, \delta_{3}$ em $k$, tal que $t_{\gamma}=\delta_{1} t_{1}+\delta_{2} t_{2}+\delta_{3} t_{3}$. Por outro lado $0=\left[e_{\gamma}, e_{\gamma}^{[2]}\right]=\gamma\left(t_{\gamma}\right) e_{\gamma}+\left[e_{\gamma}, n_{\gamma}\right]$, logo $a d\left(n_{\gamma}\right)\left(e_{\gamma}\right)=\gamma\left(t_{\gamma}\right) e_{\gamma}$, como $n_{\gamma}$ é nilpotente então $a d\left(n_{\gamma}\right)$ é nilpotente, portanto $\gamma\left(t_{\gamma}\right)=\delta_{3}=0$. Assim que, $e_{\gamma}^{[2]}=\delta_{1} t_{1}+\delta_{2} t_{2}+n_{\gamma}$. Escolhendo $\delta_{3} \neq 0$ temos que $t_{\gamma} \neq 0$ e $\alpha\left(t_{\gamma}\right)=\delta_{1} \neq 0$, logo pela proposição 2.4., $L_{\alpha} \simeq L_{\alpha+\gamma}$, isto é uma contradição, já que, $2=\operatorname{dim}_{k}\left(L_{\alpha}\right) \neq$ $\operatorname{dim}_{k}\left(L_{\alpha+\gamma}\right)=1$. Para o caso 2.3.5.l. temos que $I:=N \oplus \sum_{\xi \in \Delta} \oplus L_{\xi}$ é um ideal de $L$. Uma contradição.

- 2.3.6. Se $\operatorname{dim}(L)=15$, temos os seguintes casos:
2.3.6.a. $(15: 3,5,1,1,1,1,1,1,1)$
2.3.6.c. $(15: 3,3,2,2,1,1,1,1,1)$
2.3.6.b. $(15: 3,4,2,1,1,1,1,1,1)$
2.3.6.d. $(15: 3,2,3,2,1,1,1,1,1)$ 


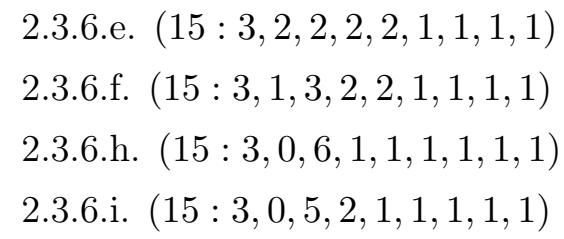

No caso 2.3.6.a., temos que $I:=N \oplus \sum_{\xi \in \Delta} \oplus L_{\xi}$ é um ideal de $L$. Uma contradição. Como no caso anterior, seja $e_{\beta}$ um elemento da base de $L_{\beta}$. Como $e_{\beta}^{[2]} \in L_{\beta}^{[2]} \subseteq$ $H=T \oplus N$, então existem $t_{\beta} \in T$ e $n_{\beta} \in N$ tal que $e_{\beta}^{[2]}=t_{\beta}+n_{\beta}$. Como $t_{\beta} \in T$ existem $\delta_{1}, \delta_{2}, \delta_{3}$ em $k$, tal que $t_{\beta}=\delta_{1} t_{1}+\delta_{2} t_{2}+\delta_{3} t_{3}$. Por outro lado $0=\left[e_{\beta}, e_{\beta}^{[2]}\right]=\beta\left(t_{\beta}\right) e_{\beta}+\left[e_{\beta}, n_{\beta}\right], \operatorname{logo} \operatorname{ad}\left(n_{\beta}\right)\left(e_{\beta}\right)=\beta\left(t_{\beta}\right) e_{\beta}$, como $n_{\beta}$ é nilpotente então $a d\left(n_{\beta}\right)$ é nilpotente, portanto $\beta\left(t_{\beta}\right)=\delta_{2}=0$. Assim que, $e_{\beta}^{[2]}=\delta_{1} t_{1}+\delta_{3} t_{3}+n_{\beta}$. Escolhendo $\delta_{1} \neq 0$ temos que $t_{\beta} \neq 0$ e $\alpha\left(t_{\beta}\right)=\delta_{1} \neq 0$, $\operatorname{logo}$ pela proposição $2.4, L_{\alpha} \simeq L_{\alpha+\beta}$. Portanto os casos 2.3.6.b. até 2.3.6.l. são impossivel, já que $\operatorname{dim}_{k}\left(L_{\alpha}\right) \neq \operatorname{dim}_{k}\left(L_{\alpha+\beta}\right)=1$.

Por outro lado, escolhendo $\delta_{1} \neq \delta_{3}$, também temos que $(\alpha+\gamma)\left(t_{\beta}\right)=(\alpha+\gamma)\left(\delta_{1} t_{1}+\delta_{3} t_{3}\right)=\delta_{1}+\delta_{3} \neq 0$, logo pela proposição 2.4 temos que $L_{\alpha+\gamma} \simeq L_{\alpha+\beta+\gamma}$, mas $2=\operatorname{dim}_{k}\left(L_{\alpha+\gamma}\right) \neq \operatorname{dim}_{k}\left(L_{\alpha+\beta+\gamma}\right)=1$, logo o caso 2.3.6. $m$. não é possivel.

- 2.3.7. Se $\operatorname{dim}_{k}(L)=16$, temos os seguintes casos:

$$
\begin{array}{ll}
\text { 2.3.7.1. }(16: 3,6,1,1,1,1,1,1,1) & 2.3 .7 .15 .(16: 3,1,4,3,1,1,1,1,1) \\
\text { 2.3.7.2. }(16: 3,5,2,1,1,1,1,1,1) & 2.3 .7 .16 .(16: 3,1,4,2,2,1,1,1,1) \\
\text { 2.3.7.3. }(16: 3,4,3,1,1,1,1,1,1) & 2.3 .7 .17 .(16: 3,1,3,3,2,1,1,1,1) \\
\text { 2.3.7.4. }(16: 3,4,2,2,1,1,1,1,1) & 2.3 .7 .18 .(16: 3,1,2,2,2,2,2,1,1) \\
\text { 2.3.7.5. }(16: 3,3,4,1,1,1,1,1,1) & 2.3 .7 .19 .(16: 3,0,7,1,1,1,1,1,1) \\
\text { 2.3.7.6. }(16: 3,3,3,2,1,1,1,1,1) & 2.3 .7 .20 .(16: 3,0,6,2,1,1,1,1,1) \\
\text { 2.3.7.7. }(16: 3,3,2,2,2,1,1,1,1) & 2.3 .7 .21 .(16: 3,0,5,2,2,1,1,1,1) \\
\text { 2.3.7.8. }(16: 3,2,5,1,1,1,1,1,1) & 2.3 .7 .22 .(16: 3,0,4,3,2,1,1,1,1) \\
\text { 2.3.7.9. }(16: 3,2,4,2,1,1,1,1,1) & 2.3 .7 .23 .(16: 3,0,4,2,2,2,1,1,1) \\
\text { 2.3.7.10. }(16: 3,2,3,3,1,1,1,1,1) & 2.3 .7 .24 .(16: 3,0,4,2,2,2,1,1,1) \\
\text { 2.3.7.11. }(16: 3,2,3,2,2,1,1,1,1) & 2.3 .7 .25 .(16: 3,0,4,2,2,2,1,1,1) \\
\text { 2.3.7.12. }(16: 3,2,2,2,2,2,1,1,1) & 2.3 .7 .26 .(16: 3,0,3,3,2,2,1,1,1) \\
2.3 .7 .13 .(16: 3,1,6,1,1,1,1,1,1) & 2.3 .7 .27 .(16: 3,0,3,2,2,2,2,1,1) \\
2.3 .7 .14 .(16: 3,1,5,2,1,1,1,1,1) & 2.3 .7 .28 .(16: 3,0,2,2,2,2,2,2,1)
\end{array}
$$

No caso 2.3.7.1., tem-se que $I:=N \oplus \sum_{\xi \in \Delta} \oplus L_{\xi}$ é um ideal de $L$, o qual é uma contradição. Novamente como nos casos anteriores, $e_{\beta}$ um elemento da base de 
2.4. ANÁLISE DE $L=H \oplus \sum_{\xi \in \Delta} \oplus L_{\xi}, C O M \Delta=T^{*} \backslash\{0\}$.

$L_{\beta}$. Como $e_{\beta}^{[2]} \in L_{\beta}^{[2]} \subseteq H=T \oplus N$, então existem $t_{\beta} \in T$ e $n_{\beta} \in N$ tal que $e_{\beta}^{[2]}=t_{\beta}+n_{\beta}$. Como $t_{\beta} \in T$ existem $\delta_{1}, \delta_{2}, \delta_{3}$ em $k$, tal que $t_{\beta}=\delta_{1} t_{1}+\delta_{2} t_{2}+\delta_{3} t_{3}$.

Por outro lado, $0=\left[e_{\beta}, e_{\beta}^{[2]}\right]=\beta\left(t_{\beta}\right) e_{\beta}+\left[e_{\beta}, n_{\beta}\right] \operatorname{logo} \operatorname{ad}\left(n_{\beta}\right)\left(e_{\beta}\right)=\beta\left(t_{\beta}\right) e_{\beta}$, como $n_{\beta}$ é nilpotente, então ad $\left(n_{\beta}\right)$ é nilpotente, portanto $\beta\left(t_{\beta}\right)=\delta_{2}=0$. Assim $e_{\beta}^{[2]}=\delta_{1} t_{1}+\delta_{3} t_{3}+n_{\beta}$. Escolhendo $\delta_{1} \neq 0$ temos que $t_{\beta} \neq 0$ e $\alpha\left(t_{\beta}\right)=\delta_{1} \neq 0$, logo pela proposição 2.4., $L_{\alpha} \cong L_{\alpha+\beta}$. Portanto os casos 2.3.7.1. até 2.3.7.27. exceto 2.3.7.12. e, 2.3.7.19. são impossivel, já que $\operatorname{dim}_{k}\left(L_{\alpha}\right) \neq \operatorname{dim}_{k}\left(L_{\alpha+\beta}\right)$

Por outro lado, escolhendo $\delta_{3} \neq 0$, também temos que $\gamma\left(t_{\beta}\right)=\delta_{3} \neq 0$, logo pela proposição 2.4. temos que $L_{\gamma} \simeq L_{\beta+\gamma}$, mas $2=\operatorname{dim}_{k}\left(L_{\gamma}\right) \neq \operatorname{dim}_{k}\left(L_{\beta+\gamma}\right)=1$, logo o caso 2.3.7.12. e 2.3.7.19. não são possivel.

No caso 2.3.7.28. escolhemos $\delta_{1} \neq \delta_{3}$, temos que $(\alpha+\gamma)\left(t_{\beta}\right)=\delta_{1}+\delta_{3} \neq 0$, logo pela proposição 2.4 temos que $L_{\alpha+\gamma} \simeq L_{\alpha+\beta+\gamma}$, o qual é absurdo já que $2=\operatorname{dim}_{k}\left(L_{\alpha+\gamma}\right) \neq \operatorname{dim}_{k}\left(L_{\alpha+\beta+\gamma}\right)=1$, portanto este caso não se dá.

A partir do corolário 2.2 e das seções 2.3 e 2.4, se obtêm o resultado seguinte.

Teorema 2.9. Não existem 2-álgebras de Lie simples de dimensão menor ou igual a 16 , de posto toral 3 .

O análise de $L=H \oplus \sum_{\xi \in \Delta} \oplus L_{\xi}, \operatorname{com} \Delta=T^{*} \backslash\{0\}$ e, $\operatorname{dim}_{k}(L)=17$ serão abordado no capítulo 4 , aí encontraremos um tipo de álgebra da forma $(17: 3,0,2,2,2,2,2,2,2)$ que precisa um estudo diferente ao apresentado nos casos quando a $\operatorname{dim}_{k}(L) \leq 16$. Para estudar esta 2-algebra de Lie, construiremos técnicas baseadas em extensões de 2-módulos sobre $\mathfrak{s l}_{3}(k)$ que seram desenvolvidas no capítulo 3 . 



\section{Capítulo 3}

\section{Extensões de $\mathfrak{s l}_{3}(k)$-2-módulos}

O objetivo principal neste capítulo é encontrar os $\left(\mathfrak{s l}_{3}(k), 2\right)$-módulos $U$ de dimensão 8 via extensões de 2 -módulos e, establecer um critério que garante a existência de um $\mathfrak{s l}_{3}(k)$-morfismo $\varphi: \Lambda^{2}(U) \rightarrow \mathfrak{s l}_{3}(k) \oplus k t_{3}$.

\subsection{Os $\mathfrak{s l}_{3}(k)$-2-módulos irredutíveis de $\operatorname{dim}_{k} \leq 8$.}

Para lograr este objetivo, començamos classificando os $\left(\mathfrak{s l}_{3}(k), 2\right)$-módulos irredutíveis de dimensão menor ou igual a 8.

Pelo exemplo 1.3, temos que $\left(\mathfrak{s l}_{3}(k), 2\right)$ é uma 2-álgebra de Lie simples de dimensão 8 , com base $\Psi:=\left\{h_{1}, h_{2}, e_{12}, e_{13}, e_{23}, e_{21}, e_{31}, e_{32}\right\}$ onde $h_{1}:=e_{11}+e_{22}, h_{2}:=e_{22}+e_{33}$. Além disso $H:=\operatorname{span}\left\{h_{1}, h_{2}\right\}$ é uma subálgebra toral de $\mathfrak{s l}_{3}(k)$, logo a decomposição em espaços de raízes de $\mathfrak{s l}_{3}(k)$ respeito a $H$ é :

$$
\mathfrak{s l}_{3}(k)=H \oplus L_{(1,0)} \oplus L_{(0,1)} \oplus L_{(1,1)}
$$

onde $L_{(1,0)}=\left\langle e_{23}, e_{32}\right\rangle, L_{(0,1)}=\left\langle e_{12}, e_{21}\right\rangle, L_{(1,1)}=\left\langle e_{13}, e_{31}\right\rangle$, com sistema de raízes $H^{*}=\{(0,0),(1,0),(0,1),(1,1)\}$ e, cada uma dessas raízes tem multiplicidade 2 .

Seja $B:=\left\{V: V\right.$ é um $\left(\mathfrak{s l}_{3}(k), 2\right)$-módulo irredutível $\}$. Então por um resultado de $\mathrm{N}$. Jacobson (Ver [SF] capítulo 5, teorema 2.4, pág. 207.) $\operatorname{dim}_{k}(V)<\infty$ para todo $V \in B$ e, $\operatorname{dim}_{k}(V) \leq 2^{4}=16$.

Nós estamos interessados em achar os $V \in B$ de dimensão menor ou igual a 8, associados aos pesos $(0,0),(1,0),(0,1),(1,1)$.

\subsubsection{2-representação irredutível de peso $(0,0)$}

Seja $\rho: \mathfrak{s l}_{3}(k) \rightarrow \mathfrak{g}(1, k)$ uma representação 1-dimensional de $\mathfrak{s l}_{3}(k)$. Então $\rho\left(\left[\mathfrak{s l}_{3}(k), \mathfrak{s l}_{3}(k)\right]\right)=0$, como $\left[\mathfrak{s l}_{3}(k), \mathfrak{s l}_{3}(k)\right]=\mathfrak{s l}_{3}(k)$, então $\rho\left(\mathfrak{s l}_{3}(k)\right)=0$. Assim, $\rho(A)=0$ para todo $A \in \mathfrak{s l}_{3}(k)$, isto é, a única representação 1-dimensional de $\mathfrak{s l}_{3}(k)$ é a representação trivial. Além disso, esta é uma 2-reprentacão de $\mathfrak{s l}_{3}(k)$ irredutível de peso $(0,0)$, e é denotada por $V_{0}$, isto é, $V_{0}:=\langle a\rangle, a \neq 0$ onde $a \cdot \mathfrak{s l}_{3}(k)=0$. 


\subsubsection{2-representação irredutível de peso $(1,0)$}

Sejam $\left\{e_{1}, e_{2}, e_{3}\right\}$ a base canónica $k^{3}$ e $\rho: \mathfrak{s l}_{3}(k) \rightarrow \mathfrak{g l}\left(k^{3}\right)$ dada por $\rho(A)(x)=x . A:=$ $x A$ a representação canónica, como $\rho\left(A^{2}\right)(x)=x \cdot A^{2}=x A^{2}=(x A) A=\rho(A)(x A)=$ $\rho(A)(x . A)=\rho(A)(\rho(A)(x))=\rho(A)^{2}(x)$, para todo $x \in k^{3}$, então $\rho\left(A^{2}\right)=\rho(A)^{2}$. Portanto, $\rho$ é uma 2-representação de $\mathfrak{s l}_{3}(k)$ em $k^{3}$. Esta 2-representação é irredutível, já que $e_{1} \cdot e_{1 i}=e_{i}$ para $i=1,2,3$ e assim $k^{3}=e_{1} \cdot \mathfrak{s l}_{3}(k)$ é gerado por $e_{1}$ como $\mathfrak{s l}_{3}(k)$ módulo. Além disso:

$$
\begin{array}{lll}
e_{1} \cdot h_{1}=e_{1} h_{1}=e_{1} & e_{2} \cdot h_{1}=e_{2} h_{1}=e_{2} & e_{3} \cdot h_{1}=e_{3} h_{1}=0 \\
e_{1} \cdot h_{2}=e_{1} h_{2}=0 & e_{2} \cdot h_{2}=e_{2} h_{2}=e_{2} & e_{3} \cdot h_{2}=e_{3} h_{2}=e_{3}
\end{array}
$$

então os vetores $e_{1}, e_{2}$ e $e_{3}$ tem peso $(1,0),(1,1)$ e $(0,1)$ respectivamente.

Assim, podemos considerar que $\rho$ é uma 2-representação isomorfa a $\mathfrak{s l}_{3}(k)$-2-módulos irredutível, $V_{3}:=\left\langle v_{1}, v_{2}, v_{3}\right\rangle$ de dimensão 3 com base $\left\{v_{1}, v_{2}, v_{3}\right\}$, onde $v_{1}, v_{2}$ e $v_{3}$ são vetores de peso $(1,0),(1,1)$ e $(0,1)$ respectivamente e, a ação de $\mathfrak{s l}_{3}(k)$ sobre $V_{3}$ é dada pela tabela seguinte:

\begin{tabular}{|c||c|c|c|c|c|c|c|c|}
\hline & $h_{1}$ & $h_{2}$ & $e_{12}$ & $e_{13}$ & $e_{23}$ & $e_{21}$ & $e_{31}$ & $e_{32}$ \\
\hline \hline$v_{1}$ & $v_{1}$ & 0 & $v_{2}$ & $v_{3}$ & 0 & 0 & 0 & 0 \\
\hline$v_{2}$ & $v_{2}$ & $v_{2}$ & 0 & 0 & $v_{3}$ & $v_{1}$ & 0 & 0 \\
\hline$v_{3}$ & 0 & $v_{3}$ & 0 & 0 & 0 & 0 & $v_{1}$ & $v_{2}$ \\
\hline
\end{tabular}

Tabela 3.2: $O \mathfrak{s l}_{3}(k)-2$-módulo irredutível $V_{3}$

\subsubsection{2-representação irredutível de peso $(0,1)$}

Consideremos a representação dual de $\rho, \rho^{*}: \mathfrak{s l}_{3}(k) \rightarrow \mathfrak{g l}\left(k^{3}\right)$ dada por:

$\rho^{*}(A)(x)=x . A:=x A^{t}$. Como $\rho^{*}\left(A^{2}\right)(x)=x\left(A^{2}\right)^{t}=x\left(A^{t} A^{t}\right)=\left(x A^{t}\right) A^{t}=\rho^{*}\left(A^{t}\right)\left(x A^{t}\right)=$ $\rho^{*}(A)\left(\rho^{*}(A)(x)\right)=\left(\rho^{*}(A)\right)^{2}(x)$, para todo $x \in \mathfrak{s l}_{3}(k)$, isto é, $\rho^{*}\left(A^{2}\right)=\left(\rho^{*}(A)\right)^{2}$. Portanto $\rho^{*}$ é uma 2-representação de $\mathfrak{s l}_{3}(k)$ em $k^{3}$. Temos que $h_{i}^{t}=h_{i}$ para $i=1,2$. Portanto, como no caso anterior temos que os pesos de $e_{1}, e_{2}$ e $e_{3}$ tem peso $(1,0),(1,1)$ e $(0,1)$ respectivamente. $\rho^{*}$ é uma representação irredutível, com efeito: $e_{3} \cdot e_{i 3}=e_{i}$, para $i=1,2,3 \operatorname{logo} k^{3}=e_{3} \cdot \mathfrak{s l}_{3}(k)$. Assim essa 2-representação irredutível, $\rho^{*}$, é isomorfa a $\mathfrak{s l}_{3}(k)$-2-módulo irreductível $V_{3}^{*}:=\left\langle w_{1}, w_{2}, w_{3}\right\rangle$ de dimensão 3 onde $\left\{w_{1}, w_{2}, w_{3}\right\}$ é uma base com $w_{1}, w_{2}$ e $w_{3}$ de pesos $(1,0),(1,1)$ e $(0,1)$ respetivamente, e a ação de $\mathfrak{s l}_{3}(k)$ sobre $V_{3}^{*}$ é dada na tabela seguinte: 


\begin{tabular}{|c||c|c|c|c|c|c|c|c|}
\hline & $h_{1}$ & $h_{2}$ & $e_{12}$ & $e_{13}$ & $e_{23}$ & $e_{21}$ & $e_{31}$ & $e_{32}$ \\
\hline \hline$w_{1}$ & $w_{1}$ & 0 & 0 & 0 & 0 & $w_{2}$ & $w_{3}$ & 0 \\
\hline$w_{2}$ & $w_{2}$ & $w_{2}$ & $w_{1}$ & 0 & 0 & 0 & 0 & $w_{3}$ \\
\hline$w_{3}$ & 0 & $w_{3}$ & 0 & $w_{1}$ & $w_{2}$ & 0 & 0 & 0 \\
\hline
\end{tabular}

Tabela 3.3: $O \mathfrak{s l}_{3}(k)-2$-módulo irredutivel $V_{3}^{*}$

Exemplo 3.1. Temos o seguinte $\mathfrak{s l}_{3}(k)$-isomorfismo de 2-módulos

$$
\mathfrak{g l}_{3}(k) \simeq V_{3} \otimes_{k} V_{3}^{*}
$$

logo pelo exemplo 1.4 temos que

$$
V_{3}^{*} \otimes_{k} V_{3} \simeq V_{3} \otimes_{k} V_{3}^{*} \simeq \mathfrak{s l}_{3}(k) \oplus \mathfrak{z}\left(\mathfrak{g l}_{3}(k)\right) \simeq \mathfrak{s l}_{3}(k) \oplus k t_{3} .
$$

onde:

$$
\begin{aligned}
V_{3} \otimes_{k} V_{3}^{*} & \stackrel{g}{\longrightarrow} \mathfrak{s l}_{3}(k) \oplus k t_{3} \\
v_{1} \otimes w_{1} & \mapsto h_{2}+t_{3} \\
v_{2} \otimes w_{2} & \mapsto h_{1}+h_{2}+t_{3} \\
v_{3} \otimes w_{3} & \mapsto h_{1}+t_{3} \\
v_{i} \otimes w_{j} & \mapsto e_{j i}
\end{aligned}
$$

$$
\begin{array}{rll}
V_{3}^{*} \otimes_{k} V_{3} & \stackrel{f}{\longrightarrow} \mathfrak{s l}_{3}(k) \oplus k t_{3} \\
v_{1} \otimes w_{1} & \mapsto h_{2}+t_{3} \\
v_{2} \otimes w_{2} & \mapsto & h_{1}+h_{2}+t_{3} \\
v_{3} \otimes w_{3} & \mapsto h_{1}+t_{3} \\
v_{i} \otimes w_{j} & \mapsto e_{i j}
\end{array}
$$

\subsubsection{2-representação irredutível de peso $(1,1)$}

Sejam $\rho: \mathfrak{s l}_{3}(k) \rightarrow \mathfrak{g l}\left(V_{3}\right)$ e $\rho^{*}: \mathfrak{s l}_{3}(k) \rightarrow \mathfrak{g l}\left(V_{3}^{*}\right)$ as 2-representações encontradas nas seções 3.1.2 e 3.1.3 respetivamente. Logo existe uma única 2-representação $\rho \otimes \rho^{*}: \mathfrak{s l}_{3}(k) \rightarrow \mathfrak{g l}\left(V_{3} \otimes_{k} V_{3}^{*}\right)$ dada por $\left(\rho \otimes \rho^{*}\right)(A)=\rho(A) \otimes I_{V_{3}^{*}}+I_{V_{3}} \otimes \rho^{*}(A)$.

Pelas tabelas 3.2, 3.3 temos:

$\left(\rho \otimes \rho^{*}\right)\left(h_{1}\right)\left(v_{1} \otimes w_{3}\right)=\left(v_{1} \otimes w_{3}\right) . h_{1}=\left(v_{1} \cdot h_{1}\right) \otimes w_{3}+v_{1} \otimes\left(w_{3} \cdot h_{1}\right)=v_{1} \otimes w_{3}+0=v_{1} \otimes w_{3}$. $\left(v_{1} \otimes w_{3}\right) \cdot h_{2}=\left(v_{1} \cdot h_{2}\right) \otimes w_{3}+v_{1} \otimes\left(w_{3} \cdot h_{2}\right)=v_{1} \otimes w_{3}$

Assim, o vetor $v_{1} \otimes w_{3}$ tem peso $(1,1)$ e é anulado pelos operadores $\left(\rho \otimes \rho^{*}\right)\left(e_{21}\right)$, $\left(\rho \otimes \rho^{*}\right)\left(e_{31}\right)$ e, $\left(\rho \otimes \rho^{*}\right)\left(e_{32}\right)$.

Seja $W$ o submódulo gerado pelas imagens sucessivas de $v_{1} \otimes w_{3}$ baixo os operadores $\left(\rho \otimes \rho^{*}\right)\left(e_{12}\right),\left(\rho \otimes \rho^{*}\right)\left(e_{13}\right)$ e, $\left(\rho \otimes \rho^{*}\right)\left(e_{23}\right)$. Como $e_{13}=\left[e_{12}, e_{23}\right]$ é suficiente só aplicar $\left(\rho \otimes \rho^{*}\right)\left(e_{12}\right)$ e $\left(\rho \otimes \rho^{*}\right)\left(e_{23}\right)$.

Agora procuramos uma base para $W$ :

$\left(\rho \otimes \rho^{*}\right)\left(e_{12}\right)\left(v_{1} \otimes w_{3}\right)=\left(v_{1} \otimes w_{3}\right) \cdot e_{12}=\left(v_{1} \cdot e_{12}\right) \otimes w_{3}+v_{1} \otimes\left(w_{3} \cdot e_{12}\right)=v_{2} \otimes w_{3}$.

$\left(\rho \otimes \rho^{*}\right)\left(e_{23}\right)\left(v_{1} \otimes w_{3}\right)=\left(v_{1} \otimes w_{3}\right) \cdot e_{23}=\left(v_{1} \cdot e_{23}\right) \otimes w_{3}+v_{1} \otimes\left(w_{3} \cdot e_{23}\right)=v_{1} \otimes w_{2}$.

$\left(\rho \otimes \rho^{*}\right)^{2}\left(e_{12}\right)\left(v_{1} \otimes w_{3}\right)=\left(v_{2} \otimes w_{3}\right) \cdot e_{12}=0 ;\left(\rho \otimes \rho^{*}\right)^{2}\left(e_{23}\right)\left(v_{1} \otimes w_{3}\right)=\left(v_{1} \otimes w_{2}\right) \cdot e_{23}=0$ $\left(\rho \otimes \rho^{*}\right)\left(e_{23}\right)\left(v_{2} \otimes w_{3}\right)=v_{3} \otimes w_{3}+v_{2} \otimes w_{2} ;\left(\rho \otimes \rho^{*}\right)\left(e_{12}\right)\left(v_{1} \otimes w_{2}\right)=v_{2} \otimes w_{2}+v_{1} \otimes w_{1}$ 
$\left(\rho \otimes \rho^{*}\right)^{2}\left(e_{12}\right)\left(v_{1} \otimes w_{2}\right)=\left(v_{2} \otimes w_{2}+v_{1} \otimes w_{1}\right) \cdot e_{12}=0 ;\left(\rho \otimes \rho^{*}\right)^{2}\left(e_{23}\right)\left(v_{3} \otimes w_{3}+v_{2} \otimes w_{2}\right)=$ $=\left(v_{3} \otimes w_{3}+v_{2} \otimes w_{2}\right) \cdot e_{23}=0$

$\left(\rho \otimes \rho^{*}\right)\left(e_{12}\right)\left(v_{3} \otimes w_{3}+v_{2} \otimes w_{2}\right)=v_{2} \otimes w_{1},\left(\rho \otimes \rho^{*}\right)\left(e_{23}\right)\left(v_{2} \otimes w_{2}+v_{1} \otimes w_{1}\right)=v_{3} \otimes w_{2} ;$

$\left(\rho \otimes \rho^{*}\right)^{2}\left(e_{12}\right)\left(v_{3} \otimes w_{3}+v_{2} \otimes w_{2}\right)=\left(v_{2} \otimes w_{1}\right) \cdot e_{12}=0 ;$

$\left(\rho \otimes \rho^{*}\right)^{2}\left(e_{23}\right)\left(v_{2} \otimes w_{2}+v_{1} \otimes w_{1}\right)=\left(v_{3} \otimes w_{2}\right) \cdot e_{23}=0$.

$\left(\rho \otimes \rho^{*}\right)\left(e_{23}\right)\left(v_{2} \otimes w_{1}\right)=v_{3} \otimes w_{1} ;\left(\rho \otimes \rho^{*}\right)\left(e_{12}\right)\left(v_{3} \otimes w_{2}\right)=v_{3} \otimes w_{1}$.

$\left(\rho \otimes \rho^{*}\right)^{2}\left(e_{23}\right)\left(v_{2} \otimes w_{1}\right)=\left(v_{3} \otimes w_{1}\right) \cdot e_{23}=0 ;\left(\rho \otimes \rho^{*}\right)^{2}\left(e_{12}\right)\left(v_{3} \otimes w_{2}\right)=\left(v_{3} \otimes w_{1}\right) \cdot e_{12}=0$.

Portanto, $W$ é um submódulo irredutível do $\mathfrak{s l}_{3}(k)$-2-módulo $V_{3} \otimes_{k} V_{3}^{*}$, gerado por $v_{1} \otimes v_{3}$, de dimensão 8 com base

$\left\{v_{1} \otimes w_{3}, v_{2} \otimes w_{3}, v_{1} \otimes w_{2}, v_{3} \otimes w_{3}+v_{2} \otimes w_{2}, v_{2} \otimes w_{2}+v_{1} \otimes w_{1}, v_{2} \otimes w_{1}, v_{3} \otimes w_{2}, v_{3} \otimes w_{1}\right\}$.

Além disso, $W$ é isomorfo a $\mathfrak{s l}_{3}(k)$-2-módulo adjunto $V^{\text {ad }}$ (ver tabela 1.1.) via o $\mathfrak{s l}_{3}(k)$-isomorfismo:

$$
\begin{aligned}
v_{2} \otimes w_{2}+v_{3} \otimes w_{3} & \mapsto h_{2} \\
v_{1} \otimes w_{1}+v_{2} \otimes w_{2} & \mapsto h_{1} \\
v_{i} \otimes w_{j} & \mapsto e_{j i}\left(\forall i, j \in I_{3} .\right)
\end{aligned}
$$

Em resumo, temos então quatro $\mathfrak{s l}_{3}(k)$-2-representações irredutíveis de dimensão menor ou igual que 8, essas são:

- Associada ao peso $(0,0)$ : temos $V_{0}$ de dimensão 1.

- Associado ao peso $(1,0)$ : temos $V_{3}$ de dimensão 3 .

- Associada ao peso $(0,1)$ : temos $V_{3}^{*}$ de dimensão 3 .

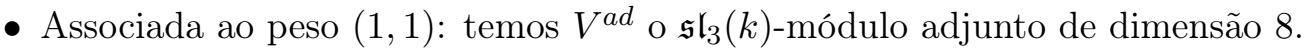

\section{$3.2 \mathfrak{s l}_{3}(k)$-2-módulos de $\operatorname{dim}_{k}<8$.}

Apartir dos $\mathfrak{s l}_{3}(k)$-2-módulos irredutíveis $V_{0}, V_{3}, V_{3}^{*}$ achados na secão 3.1., construiremos extensões de $\mathfrak{s l}_{3}(k)$-2-módulos de dimensão menor que 8 , em algumos casos se mostra por tabelas a ação de $\mathfrak{s l}_{3}(k)$ sobre uma base de cada módulo.

\subsection{1 $\mathfrak{s l}_{3}(k)$-2-módulos de $\operatorname{dim}_{k}=2$.}

Seja $E:=V_{0} \curlywedge V_{0}$ que é um $\mathfrak{s l}_{3}(k)$-2-módulos de dimensão 2 , então existe uma sequência exata curta

$$
0 \rightarrow V_{0} \stackrel{\iota}{\rightarrow} V_{0} \wedge V_{0} \stackrel{p}{\rightarrow} V_{0} \rightarrow 0
$$

tal que $V_{0}:=\langle v\rangle$ é um $\mathfrak{s l}_{3}(k)$-2-submódulos de $E$ e $E / V_{0} \simeq V_{0}$. Vejamos como é a ação de $\mathfrak{s l}_{3}(k)$ sobre $E$. Dado $a \in E / V_{0}$ existe $w \in E$ tal que $p(w)=a$. Logo, $p\left(w \cdot h_{i}\right)=a \cdot h_{i}=0, p\left(w \cdot e_{i j}\right)=a \cdot e_{i j}=0$, assim que, $w \cdot h_{i}, w \cdot e_{i j} \in \operatorname{Kerp}=\operatorname{Im}(\iota)=V_{0}$, então existe $\lambda_{i}, \lambda_{i j} \in k$ tal que $w \cdot h_{i}=\lambda_{i} v \mathrm{e}, w \cdot e_{i j}=\lambda_{i j} v$. Como $w \cdot h_{i}=w \cdot h_{i}^{2}=$ 
$\left(w \cdot h_{i}\right) \cdot h_{i}=\lambda_{i}\left(v \cdot h_{i}\right)=0, \operatorname{logo} \lambda_{i}=0$, para $i=1,2$. Por outro lado, como $w \cdot e_{i j}$ tem peso diferente de $(0,0)$ (ver observação 1.16$)$ e $v$ tem peso $(0,0)$ então $\lambda_{i j}=0$, logo $w \cdot \mathfrak{s l}_{3}(k)=0$. Por tanto, $E:=V_{0} \wedge V_{0}=\langle v, w\rangle$ é um $\mathfrak{s l}_{3}(k)$-2-módulos de dimensão 2, onde $E . \mathfrak{s l}_{3}(k)=0$ e $V_{0} \curlywedge V_{0} \simeq V_{0} \oplus V_{0}$.

\subsection{2 $\mathfrak{s l}_{3}(k)$-2-módulos de $\operatorname{dim}_{k}=4$.}

Via extensões de módulos, podemos achar os $\mathfrak{s l}_{3}(k)$-2-módulos de dimensão 4 , usando os $\mathfrak{s l}_{3}(k)$-2-módulos irredutíveis de dimensão 1 e 3 . Assim, tem-se as extensões:

1. $V_{3} \wedge V_{0}$

2. $V_{0} \curlywedge V_{3}$

3. $V_{3}^{*} \curlywedge V_{0}$

4. $V_{0} \wedge V_{3}^{*}$

Agora vamos a construir e classificar estos $\mathfrak{s l}_{3}(k)$-2-módulos de dimensão 4 :

1. Seja $E:=V_{3} \wedge V_{0}$, isto significa que existe uma sequência exata curta

$$
0 \rightarrow V_{0} \stackrel{\iota}{\rightarrow} E \stackrel{p}{\rightarrow} V_{3} \rightarrow 0
$$

onde $V_{0}:=\langle a\rangle$ é um submódulo de $E$ e $V_{3} \simeq E / V_{0}$.

Como $V_{3}:=\left\langle v_{1}, v_{2}, v_{3}\right\rangle$ existem $a_{1}, a_{2}, a_{3} \in E$ tal que $p\left(a_{i}\right)=v_{i}$.

Assim, se $p\left(a_{1}\right)=v_{1}$, então $p\left(a_{1} \cdot h_{1}\right)=p\left(a_{1}\right) \cdot h_{1}=v_{1} \cdot h_{1}=v_{1}=p\left(a_{1}\right)$, logo $\left(a_{1} \cdot h_{1}-a_{1}\right) \in \operatorname{Ker}(p)=\operatorname{Im}(\iota)=V_{0}$, então existe $\delta \in k$ tal que $a_{1} \cdot h_{1}=a_{1}+\delta a$. Também $p\left(a_{1} \cdot h_{2}\right)=p\left(a_{1}\right) \cdot h_{2}=v_{1} \cdot h_{2}=0, \operatorname{logo} a_{1} \cdot h_{2} \in \operatorname{Ker}(p)=\operatorname{Im}(\iota)=V_{0}$, então $a_{1} \cdot h_{2}=\kappa a$, para algum $\kappa \in k$.

Pela estrutura de $\mathfrak{s l}_{3}(k)$-2-módulos de $V_{3}$ temos que $a_{1} \cdot h_{2}=a_{1} \cdot h_{2}^{2}=\left(a_{1} \cdot h_{2}\right) \cdot h_{2}=$ $(\kappa a) \cdot h_{2}=\kappa\left(a \cdot h_{2}\right)=0$, portanto $\kappa=0$.

Logo temos que

$$
a_{1} \cdot h_{1}=a_{1}+\delta a, a_{1} \cdot h_{2}=0 .
$$

Da mesma forma obtemos que:

$a_{2} \cdot h_{1}=a_{2}+\lambda a, a_{2} \cdot h_{2}=a_{2}+\mu a, a_{3} \cdot h_{1}=0, a_{3} \cdot h_{2}=a_{3}+\zeta a$, para $\lambda, \mu, \zeta \in k$

Do sistema de ecuações

$$
\begin{cases}a_{1} \cdot h_{1} & =a_{1}+\delta a \\ a_{1} \cdot h_{2} & =0\end{cases}
$$

temos que $a_{1} \cdot h_{1}=\left(a_{1}+\delta_{1} a\right) \cdot h_{1}$ e $a_{1} \cdot h_{2}=\left(a_{1}+\delta_{1} a\right) \cdot h_{2}=0$. Mas,

$$
\left(a_{1}+\delta_{1} a\right) \cdot h_{1}=a_{1} \cdot h_{1}=a_{1}+\delta a
$$




$$
\left(a_{1}+\delta_{1} a\right) \cdot h_{2}=a_{1} \cdot h_{2}=0
$$

então $\left(a_{1}+\delta a\right)$ tem peso $(1,0)$. Assim que $a_{1} \cdot h_{1}$ tem peso $(1,0)$, como $h_{1}$ é de peso $(0,0)$ então $a_{1}$ é de peso $(1,0)$. Argumentando da mesma forma, encontramos que $a_{2}$ tem peso $(1,1)$ e $a_{3}$ tem peso $(0,1)$. Por outro lado, com a ajuda da tabela 3.2 temos:

$p\left(a_{1} \cdot e_{12}\right)=p\left(a_{1}\right) \cdot e_{12}=v_{1} \cdot e_{12}=v_{2}=p\left(a_{2}\right), \operatorname{logo}\left(a_{1} \cdot e_{12}-a_{2}\right) \in \operatorname{Ker}(p)=$ $\operatorname{Im}(\iota)=V_{0}$, então existe $\delta_{1} \in k$ tal que a. $a_{1} \cdot e_{12}=a_{2}+\delta_{1} a$ $p\left(a_{1} \cdot e_{13}\right)=p\left(a_{1}\right) \cdot e_{13}=v_{1} \cdot e_{13}=v_{3}=p\left(a_{3}\right), \log 0\left(a_{1} \cdot e_{13}-a_{3}\right) \in \operatorname{Ker}(p)=$ $\operatorname{Im}(\iota)=V_{0}$, então existe $\delta_{2} \in k$ tal que b. $a_{1} \cdot e_{13}=a_{3}+\delta_{2} a$

$p\left(a_{1} \cdot e_{23}\right)=p\left(a_{1}\right) \cdot e_{23}=v_{1} \cdot e_{23}=0, \operatorname{logo} a_{1} \cdot e_{23} \in \operatorname{Ker}(p)=\operatorname{Im}(\iota)=V_{0}$ então existe $\delta_{3} \in k$ tal que c. $a_{1} \cdot e_{23}=\delta_{3} a$

$p\left(a_{1} \cdot e_{21}\right)=p\left(a_{1}\right) \cdot e_{21}=v_{1} \cdot e_{21}=0, \operatorname{logo} a_{1} \cdot e_{21} \in \operatorname{Ker}(p)=\operatorname{Im}(\iota)=V_{0}$, então existe $\delta_{4} \in k$ tal que d. $a_{1} \cdot e_{21}=\delta_{4} a$

$p\left(a_{1} \cdot e_{31}\right)=p\left(a_{1}\right) \cdot e_{31}=v_{1} \cdot e_{31}=0, \log a_{1} \cdot e_{31} \in \operatorname{Ker}(p)=\operatorname{Im}(\iota)=V_{0}$, então existe $\delta_{5} \in k$ tal que e. $a_{1} \cdot e_{31}=\delta_{5} a$

$p\left(a_{1} \cdot e_{32}\right)=p\left(a_{1}\right) \cdot e_{32}=v_{1} \cdot e_{32}=0, \operatorname{logo} a_{1} \cdot e_{32} \in \operatorname{Ker}(p)=\operatorname{Im}(\iota)=V_{0}$ então existe $\delta_{6} \in k$ tal que f. $a_{1} \cdot e_{32}=\delta_{6} a$

Também temos que: $p\left(a_{2} \cdot e_{12}\right)=p\left(a_{2}\right) \cdot e_{12}=v_{2} \cdot e_{12}=0, \operatorname{logo} a_{2} \cdot e_{12} \in \operatorname{Ker}(p)=$ $\operatorname{Im}(\iota)=V_{0}$, então existe $\delta_{7} \in k$ tal que g. $a_{2} \cdot e_{12}=\delta_{7} a$.

$p\left(a_{2} \cdot e_{13}\right)=p\left(a_{2}\right) \cdot e_{13}=v_{2} \cdot e_{13}=0, \operatorname{logo} a_{2} \cdot e_{13} \in \operatorname{Ker}(p)=\operatorname{Im}(\iota)=V_{0}$, então existe $\delta_{8} \in k$ tal que h. $a_{2} . e_{13}=\delta_{8} a$

$p\left(a_{2} \cdot e_{23}\right)=p\left(a_{2}\right) \cdot e_{23}=v_{2} \cdot e_{23}=v_{3}=p\left(a_{3}\right), \log \left(a_{2} \cdot e_{23}-a_{3}\right) \in \operatorname{Ker}(p)=$ $\operatorname{Im}(\iota)=V_{0}$, então existe $\delta_{9} \in k$ tal que $\mathbf{i} a_{2} \cdot e_{23}=a_{3}+\delta_{9} a$.

$p\left(a_{2} \cdot e_{21}\right)=p\left(a_{2}\right) \cdot e_{21}=v_{2} \cdot e_{21}=v_{1}=p\left(a_{1}\right), \operatorname{logo}\left(a_{2} \cdot e_{21}-a_{1}\right) \in \operatorname{Ker}(p)=$ $\operatorname{Im}(\iota)=V_{0}$, então existe $\delta_{10} \in k$ tal que j. $a_{2} \cdot e_{21}=a_{1}+\delta_{10} a$.

$p\left(a_{2} \cdot e_{31}\right)=p\left(a_{2}\right) \cdot e_{31}=v_{2} \cdot e_{31}=0, \log 0 a_{2} \cdot e_{31} \in \operatorname{Ker}(p)=\operatorname{Im}(\iota)=V_{0}$, então existe $\delta_{11} \in k$ tal que $\mathbf{k .} a_{2} . e_{31}=\delta_{11} a$

$p\left(a_{2} \cdot e_{32}\right)=p\left(a_{2}\right) \cdot e_{32}=v_{2} \cdot e_{32}=0, \log a_{2} \cdot e_{32} \in \operatorname{Ker}(p)=\operatorname{Im}(\iota)=V_{0}$ então existe $\delta_{12} \in k$ tal que $1 . a_{2} \cdot e_{32}=\delta_{12} a$

Finalmente, temos: $p\left(a_{3} \cdot e_{12}\right)=p\left(a_{3}\right) \cdot e_{12}=v_{3} \cdot e_{12}=0, \operatorname{logo} a_{3} \cdot e_{12} \in \operatorname{Ker}(p)=$ $\operatorname{Im}(\iota)=V_{0}$, então existe $\delta_{13} \in k$ tal que $\mathbf{m} . a_{3} \cdot e_{12}=\delta_{13} a$

$p\left(a_{3} \cdot e_{13}\right)=p\left(a_{3}\right) \cdot e_{13}=v_{3} \cdot e_{13}=0, \operatorname{logo} a_{3} \cdot e_{13} \in \operatorname{Ker}(p)=\operatorname{Im}(\iota)=V_{0}$, então existe $\delta_{14} \in k$ tal que n. $a_{3} \cdot e_{13}=\delta_{14} a$

$p\left(a_{3} \cdot e_{23}\right)=p\left(a_{3}\right) \cdot e_{23}=v_{3} \cdot e_{23}=0, \log a_{3} \cdot e_{23} \in \operatorname{Ker}(p)=\operatorname{Im}(\iota)=V_{0}$, então existe $\delta_{15} \in k$ tal que o. $a_{3} \cdot e_{23}=\delta_{15} a$

$p\left(a_{3} \cdot e_{21}\right)=p\left(a_{3}\right) \cdot e_{21}=v_{3} \cdot e_{21}=0, \log a_{3} \cdot e_{21} \in \operatorname{Ker}(p)=\operatorname{Im}(\iota)=V_{0}$, então existe $\delta_{16} \in k$ tal que p. $a_{3} \cdot e_{21}=\delta_{16} a$

$p\left(a_{3} \cdot e_{31}\right)=p\left(a_{3}\right) \cdot e_{31}=v_{3} \cdot e_{31}=v_{1}=p\left(a_{1}\right), \operatorname{logo}\left(a_{3} \cdot e_{31}-a_{2}\right) \in \operatorname{Ker}(p)=$ $\operatorname{Im}(\iota)=V_{0}$, então existe $\delta_{17} \in k$ tal que q. $a_{3} \cdot e_{31}=a_{1}+\delta_{17} a$.

$p\left(a_{3} \cdot e_{32}\right)=p\left(a_{3}\right) \cdot e_{32}=v_{3} \cdot e_{32}=v_{2}=p\left(a_{2}\right), \log 0\left(a_{3} \cdot e_{32}-a_{2}\right) \in \operatorname{Ker}(p)=$ 
$\operatorname{Im}(\iota)=V_{0}$, então existe $\delta_{18} \in k$ tal que $\mathbf{r .} a_{3} \cdot e_{32}=a_{2}+\delta_{18} a$

Assim temos um sistema de 18 equações com 18 incógnitas, agora pela estrutura de $\mathfrak{s l}_{3}(k)$-2-módulos de $V_{0}$ e $V_{3}$ resolvemos este sistema para os $\delta_{i}$.

De a. temos: $0=a_{1} \cdot 0=a_{1} \cdot e_{12}^{2}=\left(\left(a_{1} \cdot e_{12}\right) \cdot e_{12}\right)=a_{2} \cdot e_{12}+\left(\delta_{1} a\right) e_{12}=a_{2} \cdot e_{12}$ logo de g. tem-se $\delta_{7}=0$. Usando este mesmo raciocínio em b., i., j., q., e, r., encontramos que: $\delta_{4}=\delta_{5}=\delta_{12}=\delta_{14}=\delta_{15}=0$. Por outro lado, de j., e a., temos $a_{2}=0+a_{2}=a_{2} \cdot e_{12}+h_{1} \cdot a_{2}=\left(a_{2} \cdot e_{12}\right) \cdot e_{21}+a_{2} \cdot\left[e_{21}, e_{12}\right]=\left(a_{2} \cdot e_{21}\right) \cdot e_{12}=$ $\left(a_{1}+\delta_{10} a\right) \cdot e_{12}=a_{1} \cdot e_{12}=a_{2}+\delta_{1} a$, então $\delta_{1} a=0$, esto é $\delta_{1}=0$. Também de r., e i., temos: $a_{3}=0+a_{3}=a_{3} \cdot e_{23}+h_{2} \cdot a_{3}=\left(a_{3} \cdot e_{23}\right) \cdot e_{32}+a_{3} \cdot\left[e_{32}, e_{23}\right]=\left(a_{3} \cdot e_{32}\right) \cdot e_{23}=$ $\left(a_{2}+\delta_{18} a\right) \cdot e_{23}=a_{2} \cdot e_{23}=a_{3}+\delta_{9} a$, então $\delta_{9} a=0$, esto é $\delta_{9}=0$. De b., e q., $a_{1}=0+a_{1}=a_{1} \cdot e_{31}+\left(h_{1}+h_{2}\right) \cdot a_{1}=\left(a_{1} \cdot e_{31}\right) e_{13}+\left[e_{31}, e_{13}\right] \cdot a_{1}=\left(a_{1} \cdot e_{13}\right) \cdot e_{31}=$ $\left(a_{3}+\delta_{2} a\right) \cdot e_{31}=a_{3} \cdot e_{31}=a_{1}+\delta_{17} a$, então $\delta_{17} a=0$, isto é $\delta_{17}=0$. Da mesma forma obtemos que : $\delta_{2}=\delta_{10}=\delta_{18}=0$.

Também de i., e j., temos que $\left(a_{2} \cdot e_{23}\right) \cdot e_{21}=\left(a_{3}+\delta_{9} a\right) \cdot e_{21}=a_{3} \cdot e_{21}$, mas $\left(a_{2} \cdot e_{23}\right) \cdot e_{21}=$ $\left(a_{2} \cdot e_{21}\right) \cdot e_{23}+a_{2} \cdot\left[e_{21}, e_{23}\right]=\left(a_{1}+\delta_{10} a\right) \cdot e_{23}+0 \cdot a_{2}=a_{1} \cdot e_{23}, \log a_{1} \cdot e_{23}=a_{3} \cdot e_{21} \mathrm{e}$ assim $\delta_{16}=\delta_{3}$. Da mesma forma obtemos que $\delta_{13}=\delta_{8}$ e $\delta_{11}=\delta_{6}$.

Sejam $\delta_{6}=\kappa=\delta_{11}, \delta_{3}=\beta=\delta_{16}$ e $\delta_{13}=\gamma=\delta_{8} \operatorname{com} \beta, \gamma, \kappa \in k$, logo a ação de $\mathfrak{s l}_{3}(k)$ sobre $E$, é dada na tabela seguinte:

\begin{tabular}{|c||c|c|c|c|c|c|c|c|}
\hline & $h_{1}$ & $h_{2}$ & $e_{12}$ & $e_{13}$ & $e_{23}$ & $e_{21}$ & $e_{31}$ & $e_{32}$ \\
\hline \hline$a$ & 0 & 0 & 0 & 0 & 0 & 0 & 0 & 0 \\
\hline$a_{1}$ & $a_{1}$ & 0 & $a_{2}$ & $a_{3}$ & $\beta a$ & 0 & 0 & $\kappa a$ \\
\hline$a_{2}$ & $a_{2}$ & $a_{2}$ & 0 & $\gamma a$ & $a_{3}$ & $a_{1}$ & $\kappa a$ & 0 \\
\hline$a_{3}$ & 0 & $a_{3}$ & $\gamma a$ & 0 & 0 & $\beta a$ & $a_{1}$ & $a_{2}$ \\
\hline
\end{tabular}

Tabela 3.4: $O \mathfrak{s l}_{3}(k)-2$-módulo $V_{3}<V_{0}$

Assim, $E:=V_{3} \curlywedge V_{0}=\left\langle a, a_{1}, a_{2}, a_{3}\right\rangle$, é um $\mathfrak{s l}_{3}(k)$-2-módulos de dimensão 4 que depende dos parametros $\beta, \gamma$ e $\kappa$, isto é, $V_{3} \wedge V_{0}=E(\beta, \gamma, \kappa) \operatorname{com} \beta, \gamma, \kappa \in k$. Agora procuremos quando $\operatorname{Ext}_{\mathfrak{s l}_{3}(k)}^{1}\left(V_{3}, V_{0}\right)=0$ ou $\operatorname{Ext}_{\mathfrak{s l}_{3}(k)}^{1}\left(V_{3}, V_{0}\right) \neq 0$.

- Se $\beta=\gamma=\kappa=0$ temos que existe $N:=\left\langle a_{1}, a_{2}, a_{3}\right\rangle$ submódulo de $E$ tal que $N \simeq V_{3}$ e $V_{0} \oplus N=E$, $\operatorname{logo} E$ cinde. Assim, $V_{3} \wedge V_{0} \simeq V_{0} \oplus V_{3}$.

- Se $\beta \neq 0, \gamma \neq 0$ ou $\kappa \neq 0$, sem perda de generalidade podemos supor que $\gamma=1$, logo temos que $V_{3} \curlywedge V_{0}:=E(\beta, \kappa)$ e $E$ não cinde. Além disso, $E(\beta, \kappa) \simeq E\left(\beta^{\prime}, \kappa^{\prime}\right) \Longleftrightarrow \beta=\beta^{\prime}$ e $\kappa=\kappa^{\prime}$.

Pelo mesmo raciocínio como no caso anterior, podemos descrever os $\mathfrak{s l}_{3}(k)$-2módulos $V_{0} \wedge V_{3}, V_{3}^{*} \curlywedge V_{0}$ e, $V_{0} \curlywedge V_{3}^{*}$ da forma seguinte:

2. $E:=V_{0}<V_{3}$. A ação de $\mathfrak{s l}_{3}(k)$ sobre $E$ é dada na tabela seguinte: 


\begin{tabular}{|c||c|c|c|c|c|c|c|c|}
\hline & $h_{1}$ & $h_{2}$ & $e_{12}$ & $e_{13}$ & $e_{23}$ & $e_{21}$ & $e_{31}$ & $e_{32}$ \\
\hline \hline$v$ & 0 & 0 & $\omega v_{3}$ & $\theta v_{2}$ & $\theta v_{1}$ & $\sigma v_{3}$ & $\sigma v_{2}$ & $\omega v_{1}$ \\
\hline$v_{1}$ & $v_{1}$ & 0 & $v_{2}$ & $v_{3}$ & 0 & 0 & 0 & 0 \\
\hline$v_{2}$ & $v_{2}$ & $v_{2}$ & 0 & 0 & $v_{3}$ & $v_{1}$ & 0 & 0 \\
\hline$v_{3}$ & 0 & $v_{3}$ & 0 & 0 & 0 & 0 & $v_{1}$ & $v_{2}$ \\
\hline
\end{tabular}

Tabela 3.5: $O \mathfrak{s l}_{3}(k)$-2-módulos $V_{0} \curlywedge V_{3}$

$\operatorname{com} \omega, \theta, \sigma \in k$.

Assim que, $E:=V_{0} \wedge V_{3}=\left\langle v, v_{1}, v_{2}, v_{3}\right\rangle$, é um $\mathfrak{s l}_{3}(k)$-2-módulos de dimensão 4 que depende dos parametros $\omega, \sigma$ e $\theta$, isto é, $V_{0} \wedge V_{3}=E(\omega, \sigma, \theta) \operatorname{com} \omega, \sigma, \theta \in k$.

- $\operatorname{Ext}_{\mathfrak{s l}_{3}(k)}^{1}\left(V_{0}, V_{3}\right)=0$. Se $\omega=\sigma=\theta=0$ temos que $E$ cinde, isto é, $E:=V_{0}<V_{3} \simeq V_{3} \oplus V_{0}$.

- $\operatorname{Ext}_{\mathfrak{s l}_{3}(k)}^{1}\left(V_{0}, V_{3}\right) \neq 0$. Se $\omega \neq 0, \sigma \neq 0$ ou $\theta \neq 0$, então sem perda de generalidade podemos supor que $\omega=1$, logo temos que $V_{0} \wedge V_{3}:=E(\sigma, \theta)$ Assim $V_{0} \prec V_{3}$ não cinde, e

$$
E(\sigma, \theta) \simeq E\left(\sigma^{\prime}, \theta^{\prime}\right) \Longleftrightarrow \sigma=\sigma^{\prime} e \theta=\theta^{\prime}
$$

3. Se $E:=V_{3}^{*} \curlywedge V_{0}$. Encontramos que a ação de $\mathfrak{s l}_{3}(k)$ sobre $E$ é dada na tabela seguinte: $\operatorname{com} \pi, \lambda, \xi \in k$.

\begin{tabular}{|c||c|c|c|c|c|c|c|c|}
\hline & $h_{1}$ & $h_{2}$ & $e_{12}$ & $e_{13}$ & $e_{23}$ & $e_{21}$ & $e_{31}$ & $e_{32}$ \\
\hline \hline$a$ & 0 & 0 & 0 & 0 & 0 & 0 & 0 & 0 \\
\hline$u_{1}$ & $u_{1}$ & 0 & 0 & 0 & $\pi a$ & $u_{2}$ & $u_{3}$ & $\lambda a$ \\
\hline$u_{2}$ & $u_{2}$ & $u_{2}$ & $u_{1}$ & $\pi a$ & 0 & 0 & $\xi a$ & $u_{3}$ \\
\hline$u_{3}$ & 0 & $u_{3}$ & $\lambda a$ & $u_{1}$ & $u_{2}$ & $\xi a$ & 0 & 0 \\
\hline
\end{tabular}

Tabela 3.6: $O \mathfrak{s l}_{3}(k)$-2-módulo $V_{3}^{*} \curlywedge V_{0}$

Assim que, $E:=V_{3}^{*} \curlywedge V_{0}=\left\langle a, u_{1}, u_{2}, u_{3}\right\rangle$, é um $\mathfrak{s l}_{3}(k)$-2-módulos de dimensão 4 que depende dos parametros $\pi, \lambda$ e $\xi$, isto é,

$$
V_{3}^{*} \curlywedge V_{0}=E(\pi, \lambda, \xi)
$$

$\operatorname{com} \pi, \lambda, \xi \in k$.

- $\operatorname{Ext}_{\mathfrak{s l}_{3}(k)}^{1}\left(V_{3}^{*}, V_{0}\right)=0$. Se $\pi=\lambda=\xi=0$ então $E$ cinde, isto é, $E:=V_{3}^{*} \curlywedge V_{0} \simeq V_{0} \oplus V_{3}^{*}$. 
- $\operatorname{Ext}_{\mathfrak{s l}_{3}(k)}^{1}\left(V_{3}^{*}, V_{0}\right) \neq 0$. Se $\pi \neq 0, \lambda \neq 0$ ou $\xi \neq 0$ podemos supor que $\pi=1$, $\operatorname{logo}$ temos que $V_{3}^{*} \curlywedge V_{0}:=E(\lambda, \xi), E$ não cinde e,

$$
E(\lambda, \xi) \simeq E\left(\lambda^{\prime}, \xi^{\prime}\right) \Longleftrightarrow \lambda=\lambda^{\prime} e \xi=\xi^{\prime} .
$$

4. Se $E:=V_{0} \wedge V_{3}^{*}$, a ação de $\mathfrak{s l}_{3}(k)$ sobre $E$ é dada na tabela seguinte: com $\delta, \alpha, \zeta \in k$

\begin{tabular}{|c||c|c|c|c|c|c|c|c|}
\hline & $h_{1}$ & $h_{2}$ & $e_{12}$ & $e_{13}$ & $e_{23}$ & $e_{21}$ & $e_{31}$ & $e_{32}$ \\
\hline \hline$w$ & 0 & 0 & $\delta w_{3}$ & $\delta w_{2}$ & $\alpha w_{1}$ & $\alpha w_{3}$ & $\zeta w_{2}$ & $\zeta w_{1}$ \\
\hline$w_{1}$ & $w_{1}$ & 0 & 0 & 0 & 0 & $w_{2}$ & $w_{3}$ & 0 \\
\hline$w_{2}$ & $w_{2}$ & $w_{2}$ & $w_{1}$ & 0 & 0 & 0 & 0 & $w_{3}$ \\
\hline$w_{3}$ & 0 & $w_{3}$ & 0 & $w_{1}$ & $w_{2}$ & 0 & 0 & 0 \\
\hline
\end{tabular}

Tabela 3.7: $O \mathfrak{s l}_{3}(k)$-2-módulos $V_{0} \curlywedge V_{3}^{*}$

Assim que, $E:=V_{0} \curlywedge V_{3}^{*}=\left\langle w, w_{1}, w_{2}, w_{3}\right\rangle$, é um $\mathfrak{s l}_{3}(k)$-2-módulos de dimensão 4 que depende dos parametros $\delta, \alpha$ e $\zeta$, isto é, $V_{0} \wedge V_{3}^{*}=E(\delta, \alpha, \zeta) \operatorname{com} \delta, \alpha, \zeta \in k$.

- $\operatorname{Ext}_{\mathfrak{s l}_{3}(k)}^{1}\left(V_{0}, V_{3}^{*}\right)=0 . \alpha=\delta=\zeta=0 \Longleftrightarrow E \simeq V_{3}^{*} \oplus V_{0}$.

- $\operatorname{Ext}_{\mathfrak{s l}_{3}(k)}^{1}\left(V_{0}, V_{3}^{*}\right)=0$. Se $\delta \neq 0, \alpha \neq 0$ ou $\zeta \neq 0$, podemos supor que $\delta=1$, logo temos que $V_{0} \wedge V_{3}^{*}:=E(\alpha, \zeta), E$ não cinde e,

$$
E(\alpha, \zeta) \simeq E\left(\alpha^{\prime}, \zeta^{\prime}\right) \Longleftrightarrow \alpha=\alpha^{\prime} \text { e } \zeta=\zeta^{\prime}
$$

3.2.3 $\mathfrak{s l}_{3}(k)$-2-módulos de $\operatorname{dim}_{k}=5$.

Usando as extensões de 2-módulos podemos construir os $\mathfrak{s l}_{3}(k)$-2-módulos de dimensão 5 , por meio dos $\mathfrak{s l}_{3}(k)$-2-módulos de dimensão 3 e 2 ou por meio dos $\mathfrak{s l}_{3}(k)$-2-módulos de dimensão 4 e 1. Logo temos os seguintes:
1. $V_{3} \wedge\left(V_{0} \wedge V_{0}\right)$
7. $\left(V_{0} \curlywedge V_{3}\right) \curlywedge V_{0}$
2. $\left(V_{0} \curlywedge V_{0}\right) \curlywedge V_{3}$
8. $V_{0} \wedge\left(V_{0} \wedge V_{3}\right)$
3. $V_{3}^{*} \curlywedge\left(V_{0} \curlywedge V_{0}\right)$
9. $\left(V_{3}^{*} \curlywedge V_{0}\right) \curlywedge V_{0}$
4. $\left(V_{0} \curlywedge V_{0}\right) \wedge V_{3}^{*}$
10. $V_{0} \curlywedge\left(V_{3}^{*} \curlywedge V_{0}\right)$
5. $\left(V_{3} \curlywedge V_{0}\right) \curlywedge V_{0}$
11. $\left(V_{0} \wedge V_{3}^{*}\right) \wedge V_{0}$
6. $V_{0} \curlywedge\left(V_{3} \wedge V_{0}\right)$
12. $V_{0} \curlywedge\left(V_{0} \wedge V_{3}^{*}\right)$

Agora, fazendo o mesmo raciocínio como na seção 3.2.2.1 podemos apresentar tabelas de ação de $\left(\mathfrak{s l}_{3}(k), 2\right)$ em alguns daqueles 2-módulos que depois precisamos. 
1. Seja $E:=V_{3} \curlywedge\left(V_{0} \curlywedge V_{0}\right)=\left\langle x, y, x_{1}, x_{2}, x_{3}\right\rangle$, a tabela de ação de $\left(\mathfrak{s l}_{3}(k), 2\right)$ sobre $E$ é :

\begin{tabular}{|c||c|c|c|c|c|c|c|c|}
\hline & $h_{1}$ & $h_{2}$ & $e_{12}$ & $e_{13}$ & $e_{23}$ & $e_{21}$ & $e_{31}$ & $e_{32}$ \\
\hline \hline$x$ & 0 & 0 & 0 & 0 & 0 & 0 & 0 & 0 \\
\hline$y$ & 0 & 0 & 0 & 0 & 0 & 0 & 0 & 0 \\
\hline$x_{1}$ & $x_{1}$ & 0 & $x_{2}$ & $x_{3}$ & $\tau_{1} x+\tau_{2} y$ & 0 & 0 & $\tau_{3} x+\tau_{4} y$ \\
\hline$x_{2}$ & $x_{2}$ & $x_{2}$ & 0 & $\tau_{5} x+\tau_{6} y$ & $x_{3}$ & $x_{1}$ & $\tau_{7} x+\tau_{8} y$ & 0 \\
\hline$x_{3}$ & 0 & $x_{3}$ & $\tau_{9} x+\tau_{10} y$ & 0 & 0 & $\tau_{11} x+\tau_{12} y$ & $x_{1}$ & $x_{2}$ \\
\hline
\end{tabular}

Tabela 3.8: $O \mathfrak{s l}_{3}(k)-2-$ módulo $V_{3} \curlywedge\left(V_{0} \wedge V_{0}\right)$

$\operatorname{com} \tau_{i} \in k, i=1, \ldots, 12$.

$$
E \simeq\left(V_{0} \curlywedge V_{0}\right) \oplus V_{3} \Longleftrightarrow \tau_{i}=0 \forall i=1, \ldots, 12
$$

3. Seja $E:=V_{3}^{*} \wedge\left(V_{0} \wedge V_{0}\right)=\left\langle v, w, w_{1}, w_{2}, w_{3}\right\rangle$, a tabela de ação de $\left(\mathfrak{s l}_{3}(k), 2\right)$ sobre $E$ é dada na tabela a seguir:

\begin{tabular}{|c||c|c|c|c|c|c|c|c|}
\hline & $h_{1}$ & $h_{2}$ & $e_{12}$ & $e_{13}$ & $e_{23}$ & $e_{21}$ & $e_{31}$ & $e_{32}$ \\
\hline \hline$v$ & 0 & 0 & 0 & 0 & 0 & 0 & 0 & 0 \\
\hline$w$ & 0 & 0 & 0 & 0 & 0 & 0 & 0 & 0 \\
\hline$w_{1}$ & $w_{1}$ & 0 & 0 & 0 & $\chi_{1} v+\chi_{2} w$ & $w_{2}$ & $w_{3}$ & $\chi_{3} v+\chi_{4} w$ \\
\hline$w_{2}$ & $w_{2}$ & $w_{2}$ & $w_{1}$ & $\chi_{1} v+\chi_{2} w$ & 0 & 0 & $\chi_{5} v+\chi_{6} w$ & $w_{3}$ \\
\hline$w_{3}$ & 0 & $w_{3}$ & $\chi_{3} v+\chi_{4} w$ & $w_{1}$ & $w_{2}$ & $\chi_{5} v+\chi_{6} w$ & 0 & 0 \\
\hline
\end{tabular}

Tabela 3.9: $O \mathfrak{s l}_{3}(k)-2-m o ́ d u l o s ~ E:=V_{3}^{*} \curlywedge\left(V_{0} \curlywedge V_{0}\right)$

$\operatorname{com} \chi_{i} \in k$.

Daí concluimos que:

$$
E \simeq\left(V_{0} \wedge V_{0}\right) \oplus V_{3}^{*} \Longleftrightarrow \chi_{i}=0, \quad \forall i=1, \ldots, 6 .
$$

\subsection{4 $\mathfrak{s l}_{3}(k)$-2-módulos de $\operatorname{dim}_{k}=6$.}

Por meio das extensões de módulos e usando os $\mathfrak{s l}_{3}(k)$-2-módulos de dimensão $3 \mathrm{ou}$, $\mathfrak{s l}_{3}(k)$-2-módulos de dimensão 2 e 4 , ou $\mathfrak{s l}_{3}(k)$-2-módulos de dimensão 1 e 5 , obtemos os seguintes $\mathfrak{s l}_{3}(k)$-2-módulos de dimensão 6 .
1. $V_{3}^{*} \curlywedge V_{3}$
4. $V_{3}^{*} \curlywedge V_{3}^{*}$
2. $V_{3} \wedge V_{3}$
5. $\left(V_{3} \curlywedge V_{0}\right) \curlywedge\left(V_{0} \curlywedge V_{0}\right)$
3. $V_{3}<V_{3}^{*}$
6. $\left(V_{0} \curlywedge V_{0}\right) \curlywedge\left(V_{3} \curlywedge V_{0}\right)$ 

7. $\left(V_{0} \curlywedge V_{3}\right) \curlywedge\left(V_{0} \curlywedge V_{0}\right)$
22. $\left[V_{0} \curlywedge\left(V_{3}^{*} \curlywedge V_{0}\right)\right] \curlywedge V_{0}$
8. $\left(V_{0}<V_{0}\right) \curlywedge\left(V_{0}<V_{3}\right)$
23. $\left.\left[\left(V_{0} \curlywedge V_{3}^{*}\right) \curlywedge V_{0}\right)\right] \curlywedge V_{0}$
9. $\left(V_{3}^{*} \curlywedge V_{0}\right) \curlywedge\left(V_{0} \curlywedge V_{0}\right)$
24. $\left[V_{0} \curlywedge\left(V_{0} \curlywedge V_{3}^{*}\right)\right] \curlywedge V_{0}$
10. $\left(V_{0} \curlywedge V_{0}\right) \curlywedge\left(V_{3}^{*} \curlywedge V_{0}\right)$
25. $V_{0} \curlywedge\left[V_{3} \curlywedge\left(V_{0} \curlywedge V_{0}\right)\right]$
11. $\left(V_{0} \curlywedge V_{3}^{*}\right) \curlywedge\left(V_{0} \curlywedge V_{0}\right)$
26. $\left.V_{0} \curlywedge\left[\left(V_{0} \wedge V_{0} \curlywedge\right) V_{3}\right)\right]$
12. $\left(V_{0} \wedge V_{0}\right) \curlywedge\left(V_{0} \wedge V_{3}^{*}\right)$
27. $V_{0} \wedge\left[V_{3}^{*} \curlywedge\left(V_{0} \wedge V_{0}\right)\right]$
13. $\left[V_{3} \curlywedge\left(V_{0} \curlywedge V_{0}\right)\right] \curlywedge V_{0}$
28. $\left.V_{0} \curlywedge\left[\left(V_{0} \wedge V_{0}\right) \wedge V_{3}^{*}\right)\right]$
14. $\left[\left(V_{0} \wedge V_{0}\right) \wedge V_{3}\right] \wedge V_{0}$
29. $\left.V_{0} \curlywedge\left[\left(V_{3} \wedge V_{0}\right) \curlywedge V_{0}\right)\right]$
15. $\left[V_{3}^{*} \curlywedge\left(V_{0} \curlywedge V_{0}\right)\right] \curlywedge V_{0}$
30. $V_{0} \nprec\left[V_{0} \curlywedge\left(V_{3} \prec V_{0}\right)\right]$
16. $\left.\left[\left(V_{0} \curlywedge V_{0}\right) \curlywedge V_{3}^{*}\right)\right] \curlywedge V_{0}$
31. $\left.V_{0} \curlywedge\left[\left(V_{0} \curlywedge V_{3}\right) \curlywedge V_{0}\right)\right]$
17. $\left.\left[\left(V_{3} \wedge V_{0}\right) \wedge V_{0}\right)\right] \curlywedge V_{0}$
32. $V_{0} \curlywedge\left[V_{0} \curlywedge\left(V_{0} \prec V_{3}\right)\right]$
18. $\left[V_{0} \curlywedge\left(V_{3} \wedge V_{0}\right)\right] \curlywedge V_{0}$
33. $\left.V_{0} \wedge\left[\left(V_{3}^{*} \wedge V_{0}\right) \wedge V_{0}\right)\right]$
19. $\left.\left[\left(V_{0} \wedge V_{3}\right) \wedge V_{0}\right)\right] \wedge V_{0}$
34. $V_{0} \curlywedge\left[V_{0} \curlywedge\left(V_{3}^{*} \curlywedge V_{0}\right)\right]$
20. $\left[V_{0} \curlywedge\left(V_{0} \curlywedge V_{3}\right)\right] \curlywedge V_{0}$
35. $\left.V_{0} \curlywedge\left[\left(V_{0} \wedge V_{3}^{*}\right) \curlywedge V_{0}\right)\right]$
21. $\left[\left(V_{3}^{*} \wedge V_{0}\right) \wedge V_{0}\right] \curlywedge V_{0}$
36. $V_{0} \curlywedge\left[V_{0} \curlywedge\left(V_{0} \curlywedge V_{3}^{*}\right)\right]$

Agora descreveremos as extensões dos $\mathfrak{s l}_{3}(k)$-2-módulos 1 e 3 da lista anterior.

1. Seja $E:=V_{3}^{*} \wedge V_{3}:=\left\langle v_{1}, v_{2}, v_{3}, u_{1}, u_{2}, u_{3}\right\rangle$ um $\mathfrak{s l}_{3}(k)$-2-módulos de dimensão 6 ; lembre que a notação $V_{3}^{*} \wedge V_{3}$ indica que $V_{3}=\left\langle v_{1}, v_{2}, v_{3}\right\rangle$ é um $\mathfrak{s l}_{3}(k)$-2-submódulos de $E$ e $E / V_{3} \simeq V_{3}^{*}$, portanto temos a sequência exata curta:

$$
0 \rightarrow V_{3} \stackrel{\iota}{\rightarrow} V_{3}^{*}<V_{3} \stackrel{p}{\rightarrow} V_{3}^{*} \rightarrow 0
$$

Vejamos como é a ação de $\mathfrak{s l}_{3}(k)$ sobre $E$. Como $V_{3}^{*}:=\left\langle w_{1}, w_{2}, w_{3}\right\rangle$ existem $u_{1}, u_{2}, u_{3} \in E$ tal que $p\left(u_{i}\right)=w_{i}$. Logo temos que:

$p\left(u_{1} \cdot e_{12}\right)=p\left(u_{1}\right) \cdot e_{12}=w_{1} \cdot e_{12}=0$, então $u_{1} \cdot e_{12} \in \operatorname{Ker}(p)=\operatorname{Im}(\iota)=V_{3}$, como $u_{1} . e_{12}$ é de peso $(1,1)$, então existe $\delta_{1} \in k$ tal que $u_{1} \cdot e_{12}=\delta_{1} v_{2}$.

$p\left(u_{1} \cdot e_{13}\right)=p\left(u_{1}\right) \cdot e_{13}=w_{1} \cdot e_{13}=0$, então $u_{1} \cdot e_{13} \in \operatorname{Ker}(p)=\operatorname{Im}(\iota)=V_{3}$, como $u_{1} . e_{13}$ é de peso $(0,1)$, então existe $\delta_{2} \in k$ tal que $u_{1} \cdot e_{13}=\delta_{2} v_{3}$. $p\left(u_{1} \cdot e_{23}\right)=p\left(u_{1}\right) \cdot e_{23}=w_{1} \cdot e_{23}=0$, então $u_{1} \cdot e_{23} \in \operatorname{Ker}(p)=\operatorname{Im}(\iota)=V_{3}$, como $u_{1} \cdot e_{23}$ é de peso $(0,0)$, então $u_{1} \cdot e_{23}=0$.

$p\left(u_{1} \cdot e_{21}\right)=p\left(u_{1}\right) \cdot e_{21}=w_{1} \cdot e_{21}=w_{2}=\rho\left(u_{2}\right)$, então $\left(u_{1} \cdot e_{21}-u_{2}\right) \in \operatorname{Ker}(p)=$ $\operatorname{Im}(\iota)=V_{3}$, como $u_{1} \cdot e_{21}$ é de peso $(1,1)$, então existe $\delta_{3} \in k$ tal que $u_{1} \cdot e_{21}=u_{2}+\delta_{3} v_{2}$. 
$p\left(u_{1} \cdot e_{31}\right)=p\left(u_{1}\right) \cdot e_{31}=w_{1} \cdot e_{31}=w_{3}=\rho\left(u_{3}\right)$, então $\left(u_{1} \cdot e_{31}-u_{3}\right) \in \operatorname{Ker}(p)=$ $\operatorname{Im}(\iota)=V_{3}$, como $u_{1} . e_{31}$ é de peso $(0,1)$, então existe $\delta_{4} \in k$ tal que $u_{1} \cdot e_{31}=u_{3}+\delta_{4} v_{3}$. $p\left(u_{1} \cdot e_{32}\right)=p\left(u_{1}\right) \cdot e_{32}=w_{1} \cdot e_{32}=0$, então $u_{1} \cdot e_{32} \in \operatorname{Ker}(p)=\operatorname{Im}(\iota)=V_{3}$, como $u_{1} \cdot e_{32}$ é de peso $(0,0)$, então $u_{1} \cdot e_{32}=0$.

$p\left(u_{2} \cdot e_{12}\right)=p\left(u_{2}\right) \cdot e_{12}=w_{2} \cdot e_{12}=w_{1}=\rho\left(u_{1}\right)$, então $\left(u_{2} \cdot e_{12}-u_{1}\right) \in \operatorname{Ker}(p)=$ $\operatorname{Im}(\iota)=V_{3}$, como $u_{2} . e_{12}$ é de peso $(1,0)$ então existe $\delta_{5} \in k$ tal que $u_{2} . e_{12}=u_{1}+\delta_{5} v_{1}$. $p\left(u_{2} \cdot e_{13}\right)=p\left(u_{2}\right) \cdot e_{13}=w_{2} \cdot e_{13}=0$ então $u_{2} \cdot e_{13} \in \operatorname{Ker}(p)=\operatorname{Im}(\iota)=V_{3}$, como $u_{2} \cdot e_{13}$ é de peso $(0,0)$, então $u_{2} \cdot e_{13}=0$.

$p\left(u_{2} \cdot e_{23}\right)=p\left(u_{2}\right) \cdot e_{23}=w_{2} \cdot e_{23}=0$, então $u_{2} \cdot e_{23} \in \operatorname{Ker}(p)=\operatorname{Im}(\iota)=V_{3}$, como $u_{2} . e_{23}$ é de peso $(0,1)$, então existe $\delta_{6} \in k$ tal que $u_{2} \cdot e_{23}=\delta_{6} v_{3}$.

$p\left(u_{2} \cdot e_{21}\right)=p\left(u_{2}\right) \cdot e_{21}=w_{2} \cdot e_{21}=0$, então $u_{2} \cdot e_{21} \in \operatorname{Ker}(p)=\operatorname{Im}(\iota)=V_{3}$, como $u_{2} . e_{21}$ é de peso $(1,0)$, então existe $\delta_{7} \in k$ tal que $u_{2} \cdot e_{21}=\delta_{7} v_{1}$ $p\left(u_{2} \cdot e_{31}\right)=p\left(u_{2}\right) \cdot e_{31}=w_{2} \cdot e_{31}=0$, então $u_{2} \cdot e_{31} \in \operatorname{Ker}(p)=\operatorname{Im}(\iota)=V_{3}$, como $u_{2} . e_{31}$ é de peso $(0,0)$, então $u_{2} \cdot e_{31}=0$

$p\left(u_{2} \cdot e_{32}\right)=p\left(u_{2}\right) \cdot e_{32}=w_{2} \cdot e_{32}=w_{3}=\rho\left(u_{3}\right)$ então $\left(u_{2} . e_{32}-u_{3}\right) \in \operatorname{Ker}(p)=$ $\operatorname{Im}(\iota)=V_{3}$, como $u_{2} . e_{32}$ é de peso $(0,1)$, então existe $\delta_{8} \in k$ tal que $u_{2} \cdot e_{32}=u_{3}+\delta_{8} v_{3}$. $p\left(u_{3} \cdot e_{12}\right)=p\left(u_{3}\right) \cdot e_{12}=w_{3} \cdot e_{12}=0$, então $u_{3} \cdot e_{12} \in \operatorname{Ker}(p)=\operatorname{Im}(\iota)=V_{3}$, como $u_{3} . e_{12}$ é de peso $(0,0)$, então $u_{3} \cdot e_{12}=0$

$p\left(u_{3} \cdot e_{13}\right)=p\left(u_{3}\right) \cdot e_{13}=w_{3} \cdot e_{13}=w_{1}=\rho\left(u_{1}\right)$, então $\left(u_{3} \cdot e_{13}-u_{1}\right) \in \operatorname{Ker}(p)=$ $\operatorname{Im}(\iota)=V_{3}$, como $u_{3} \cdot e_{13}$ é de peso $(1,0)$, então existe $\delta_{9} \in k$ tal que $u_{3} \cdot e_{13}=u_{1}+\delta_{9} v_{1}$. $p\left(u_{3} \cdot e_{23}\right)=p\left(u_{3}\right) \cdot e_{23}=w_{3} \cdot e_{23}=w_{2}=\rho\left(u_{2}\right)$, então $\left(u_{3} \cdot e_{23}-u_{2}\right) \in \operatorname{Ker}(p)=$ $\operatorname{Im}(\iota)=V_{3}$, como $u_{3} . e_{23}$ é de peso $(1,1)$, então existe $\delta_{10} \in k$ tal que $u_{3} \cdot e_{23}=u_{2}+\delta_{10} v_{2}$ $p\left(u_{3} \cdot e_{21}\right)=p\left(u_{3}\right) \cdot e_{21}=w_{3} \cdot e_{21}=0$, então $u_{3} \cdot e_{21} \in \operatorname{Ker}(p)=\operatorname{Im}(\iota)=V_{3}$, como $u_{3} \cdot e_{21}$ é de peso $(0,0)$, então $u_{3} \cdot e_{21}=0$.

$p\left(u_{3} \cdot e_{31}\right)=p\left(u_{3}\right) \cdot e_{31}=w_{3} \cdot e_{31}=0$, então $u_{3} \cdot e_{31} \in \operatorname{Ker}(p)=\operatorname{Im}(\iota)=V_{3}$, como $u_{3} . e_{31}$ é de peso $(0,1)$, então existe $\delta_{1} 1 \in k$ tal que $u_{3} \cdot e_{31}=\delta_{1} 1 v_{3}$.

$p\left(u_{3} \cdot e_{32}\right)=p\left(u_{3}\right) \cdot e_{32}=w_{3} \cdot e_{32}=0$, então $u_{3} . e_{32} \in \operatorname{Ker}(p)=\operatorname{Im}(\iota)=V_{3}$, como $u_{3} . e_{32}$ é de peso $(1,1)$, então existe $\delta_{12} \in k$ tal que $u_{3} \cdot e_{32}=\delta_{12} v_{2}$.

Agora, pela estrutura de $\mathfrak{s l}_{3}(k)$-2-módulos de $V_{3}$ e $V_{3}^{*}$, podemos calcular os $\delta_{i}$ : $\left(u_{1} \cdot e_{13}\right) \cdot e_{31}=\delta_{2} v_{3} \cdot e_{31}=\delta_{2} v_{1}$, como $\left(u_{1} \cdot e_{31}\right) \cdot e_{13}=\left(u_{1} \cdot e_{13}\right) \cdot e_{31}+u_{1} \cdot\left(h_{1}+h_{2}\right)=$ $u_{3} . e_{13}+u_{1}=u_{1}+\delta_{9} v_{1}+u_{1}=\delta_{9} v_{1}$, então $\delta_{2}=\delta_{9}$.

$\left(u_{1} \cdot e_{12}\right) \cdot e_{21}=\delta_{1} v_{2} \cdot e_{21}=\delta_{1} v_{1}$, como $\left(u_{1} \cdot e_{12}\right) \cdot e_{21}=\left(u_{1} \cdot e_{21}\right) \cdot e_{12}+u_{1} \cdot h_{1}=u_{2} \cdot e_{12}+$ $u_{1}=u_{1}+\delta_{1} v_{1}+u_{1}=\delta_{1} v_{1}$, então $\delta_{1}=\delta_{5}$.

$\left(u_{1} \cdot e_{12}\right) \cdot e_{23}=\delta_{1} v_{2} \cdot e_{23}=\delta_{1} v_{3}$, como $\left(u_{1} \cdot e_{12}\right) \cdot e_{23}=\left(u_{1} \cdot e_{23}\right) \cdot e_{12}+u_{1} \cdot e_{13}=\delta_{2} v_{3}$, então $\delta_{1}=\delta_{2}$.

$0=\left(u_{1} \cdot e_{21}\right) \cdot e_{21}=u_{2} \cdot e_{21}+\delta_{3} v_{2} \cdot e_{21}=\delta_{7} v_{1}+\delta_{3} v_{1}$, então $\delta_{7}=\delta_{3}$. $\left(u_{2} \cdot e_{23}\right) \cdot e_{31}=\delta_{6} v_{3} \cdot e_{31}=\delta_{6} v_{1}$, como $\left(u_{2} \cdot e_{23}\right) \cdot e_{31}=\left(u_{2} \cdot e_{31}\right) \cdot e_{23}+u_{2} \cdot e_{21}=\delta_{7} v_{1}$, então $\delta_{7}=\delta_{6}$.

$\left(u_{2} \cdot e_{23}\right) \cdot e_{32}=\delta_{6} v_{3} \cdot e_{32}=\delta_{6} v_{2}, \operatorname{como}\left(u_{2} \cdot e_{23}\right) \cdot e_{32}=\left(u_{2} \cdot e_{32}\right) \cdot e_{23}+u_{2} \cdot h_{2}=u_{3} \cdot e_{23}+$ $u_{2}=u_{2}+\delta_{10} v_{2}+u_{2}=\delta_{10} v_{2}$, então $\delta_{10}=\delta_{6}$.

$\left(u_{1} \cdot e_{21}\right) \cdot e_{32}=u_{2} \cdot e_{32}+\delta_{3} v_{2} \cdot e_{32}=u_{3}+\delta_{8} v_{3}$, como $\left(u_{1} \cdot e_{21}\right) \cdot e_{32}=\left(u_{1} \cdot e_{32}\right) \cdot e_{21}+$ 
$u_{1} e_{31}=u_{1} \cdot e_{31}=u_{3}+\delta_{4} v_{3}$, então $\delta_{4}=\delta_{8}$.

$\left(u_{3} \cdot e_{31}\right) \cdot e_{12}=\delta_{11} v_{1} \cdot e_{12}=\delta_{11} v_{2}, \operatorname{como}\left(u_{3} \cdot e_{31}\right) \cdot e_{12}=\left(u_{3} \cdot e_{12}\right) \cdot e_{31}+u_{3} \cdot e_{32}=\delta_{12} v_{2}$, então $\delta_{11}=\delta_{12}$.

$0=\left(u_{1} \cdot e_{31}\right) \cdot e_{31}=u_{3} \cdot e_{31}+\delta_{4} v_{3} \cdot e_{31}=\delta_{1} 1 v_{3}+\delta_{4} v_{1}$, então $\delta_{4}=\delta_{11}$.

Agora fazemos $\alpha:=\delta_{1}=\delta_{2}=\delta_{5}=\delta_{9}, \beta:=\delta_{4}=\delta_{8}=\delta_{11}=\delta_{12}$, e $\gamma:=\delta_{3}=\delta_{6}=\delta_{7}=\delta_{10} \operatorname{com} \alpha, \beta, \gamma \in k$.

Assim, la ação de $\mathfrak{s l}_{3}(k)$ sobre $E$ é dada tabela seguinte.

\begin{tabular}{|c||c|c|c|c|c|c|c|c|}
\hline & $h_{1}$ & $h_{2}$ & $e_{12}$ & $e_{13}$ & $e_{23}$ & $e_{21}$ & $e_{31}$ & $e_{32}$ \\
\hline \hline$v_{1}$ & $v_{1}$ & 0 & $v_{2}$ & $v_{3}$ & 0 & 0 & 0 & 0 \\
\hline$v_{2}$ & $v_{2}$ & $v_{2}$ & 0 & 0 & $v_{3}$ & $v_{1}$ & 0 & 0 \\
\hline$v_{3}$ & 0 & $v_{3}$ & 0 & 0 & 0 & 0 & $v_{1}$ & $v_{2}$ \\
\hline$u_{1}$ & $u_{1}$ & 0 & $\alpha v_{2}$ & $\alpha v_{3}$ & 0 & $u_{2}+\gamma v_{2}$ & $u_{3}+\beta v_{3}$ & 0 \\
\hline$u_{2}$ & $u_{2}$ & $u_{2}$ & $u_{1}+\alpha v_{1}$ & 0 & $\gamma v_{3}$ & $\gamma v_{1}$ & 0 & $u_{3}+\beta v_{3}$ \\
\hline$u_{3}$ & 0 & $u_{3}$ & 0 & $u_{1}+\alpha v_{1}$ & $u_{2}+\gamma v_{2}$ & 0 & $\beta v_{1}$ & $\beta v_{2}$ \\
\hline
\end{tabular}

Tabela 3.10: $O \mathfrak{s l}_{3}(k)$-2-módulos $V_{3}^{*} \curlywedge V_{3}$

Dessa tabela vemos que:

$E:=V_{3}^{*} \curlywedge V_{3}$, é um $\mathfrak{s l}_{3}(k)$-2-módulos de dimensão 6 que depende dos parametros $\alpha, \beta$ e $\gamma$, isto é, $V_{3}^{*} \wedge V_{3}=E(\alpha, \beta, \gamma)$ com $\alpha, \beta, \gamma \in k$. Além disso:

- $\operatorname{Ext}_{\mathfrak{s l}_{3}(k)}^{1}\left(V_{3}^{*}, V_{3}\right)=0$. Se $\alpha=\beta=\gamma=0$ temos que $\left\langle u_{1}, u_{2}, u_{3}\right\rangle \simeq V_{3}^{*}, \operatorname{logo}$ $E$ cinde, isto é, $E:=V_{3}^{*} \wedge V_{3} \simeq V_{3}^{*} \oplus V_{3}$.

- $\operatorname{Ext}_{\mathfrak{s l}_{3}(k)}^{1}\left(V_{3}^{*}, V_{3}\right) \neq 0$. Se $\alpha \neq 0, \beta \neq 0$ ou $\gamma \neq 0$, sem perda de generalidade podemos supor que $\gamma=1$, logo temos que $V_{3}^{*} \wedge V_{3}:=E(\alpha, \beta)$ não cinde e $E(\alpha, \beta) \simeq E\left(\alpha^{\prime}, \beta^{\prime}\right) \Longleftrightarrow \exists t \in k^{*}$ tal que $\alpha=\alpha^{\prime} t$ e $\beta=\beta^{\prime} t$.

3. Seja $E:=V_{3} \wedge V_{3}^{*}=\left\langle w_{1}, w_{2}, w_{3}, u_{1}, u_{2}, u_{3}\right\rangle$, um $\mathfrak{s l}_{3}(k)$-2-módulo de dimensão 6 onde $V_{3}^{*}=\left\langle w_{1}, w_{2}, w_{3}\right\rangle$ é $\mathfrak{s l}_{3}(k)$-2-submódulo de $E$ e $u_{1}, u_{2}, u_{3}$ tem peso $(1,0),(1,1),(0,1)$ respectivamente. Argumentando da mesma forma como no caso anterior, 3.2.4.1., temos que a ação de $\mathfrak{s l}_{3}(k)$ sobre $E$ é dada na tabela seguinte:

\begin{tabular}{|c||c|c|c|c|c|c|c|c|}
\hline & $h_{1}$ & $h_{2}$ & $e_{12}$ & $e_{13}$ & $e_{23}$ & $e_{21}$ & $e_{31}$ & $e_{32}$ \\
\hline \hline$w_{1}$ & $w_{1}$ & 0 & 0 & 0 & 0 & $w_{2}$ & $w_{3}$ & 0 \\
\hline$w_{2}$ & $w_{2}$ & $w_{2}$ & $w_{1}$ & 0 & 0 & 0 & 0 & $w_{3}$ \\
\hline$w_{3}$ & 0 & $w_{3}$ & 0 & $w_{1}$ & $w_{2}$ & 0 & 0 & 0 \\
\hline$u_{1}$ & $u_{1}$ & 0 & $u_{2}+\alpha w_{2}$ & $u_{3}+\gamma w_{3}$ & 0 & $\beta w_{2}$ & $\beta w_{3}$ & 0 \\
\hline$u_{2}$ & $u_{2}$ & $u_{2}$ & $\alpha w_{1}$ & 0 & $u_{3}+\gamma w_{3}$ & $u_{1}+\beta w_{1}$ & 0 & $\alpha w_{3}$ \\
\hline$u_{3}$ & 0 & $u_{3}$ & 0 & $\gamma w_{1}$ & $\gamma w_{2}$ & 0 & $u_{1}+\beta w_{1}$ & $u_{2}+\alpha w_{2}$ \\
\hline
\end{tabular}

Tabela 3.11: $O \mathfrak{s l}_{3}(k)-2$-módulos $V_{3} \curlywedge V_{3}^{*}$ 
Assim, $E:=V_{3}<V_{3}^{*}$, é um $\mathfrak{s l}_{3}(k)$-2-módulos de dimensão 6 que depende dos parametros $\alpha, \beta$ e $\gamma$, isto é, $V_{3} \wedge V_{3}^{*}=E(\alpha, \beta, \gamma) \operatorname{com} \alpha, \beta, \gamma \in k$.

- $\operatorname{Ext}_{\mathfrak{s l}_{3}(k)}^{1}\left(V_{3}, V_{3}^{*}\right)=0$. Quando $\alpha=\beta=\gamma=0$ temos que $<u_{1}, u_{2}, u_{3}>\simeq V_{3}$, $\operatorname{logo} E$ cinde, isto é, $E:=V_{3}<V_{3}^{*} \simeq V_{3} \oplus V_{3}^{*}$

- $\operatorname{Ext}_{\mathfrak{s l}_{3}(k)}^{1}\left(V_{3}, V_{3}^{*}\right) \neq 0$. Quando $\alpha \neq 0, \beta \neq 0$ ou $\gamma \neq 0$, sem perda de generalidade podemos supor que $\gamma=1$, logo temos que $V_{3} \wedge V_{3}^{*}:=E(\alpha, \beta)$ e $E(\alpha, \beta) \simeq E\left(\alpha^{\prime}, \beta^{\prime}\right) \Longleftrightarrow \exists t \in k^{*}$ tal que $\alpha=\alpha^{\prime} t$ e $\beta=\beta^{\prime} t$.

\subsection{5 $\mathfrak{s l}_{3}(k)$-2-módulos de $\operatorname{dim}_{k}=7$.}

Podemos construir $\mathfrak{s l}_{3}(k)$-2-módulos de dimensão 7 por meio de extensões de módulos, usando os $\mathfrak{s l}_{3}(k)$-2-módulos de dimensão 3 e 4 , ou de dimensão 5 e 2 , ou de dimensão 6 e 1, os quales seram apresentados na lista seguinte:
1. $\left(V_{3}<V_{0}\right) \wedge V_{3}$
20. $\left[\left(V_{0} \wedge V_{0}\right) \curlywedge V_{3}^{*}\right] \curlywedge\left(V_{0} \curlywedge V_{0}\right)$
2. $V_{3} \curlywedge\left(V_{3} \curlywedge V_{0}\right)$
21. $\left[\left(V_{3} \curlywedge V_{0}\right) \curlywedge V_{0}\right] \curlywedge\left(V_{0} \curlywedge V_{0}\right)$
3. $\left(V_{3} \curlywedge V_{0}\right) \curlywedge V_{3}^{*}$
22. $\left[V_{0} \curlywedge\left(V_{3} \wedge V_{0}\right)\right] \curlywedge\left(V_{0} \wedge V_{0}\right)$
4. $V_{3}^{*} \curlywedge\left(V_{3} \wedge V_{0}\right)$
23. $\left[\left(V_{0} \curlywedge V_{3}\right) \wedge V_{0}\right] \curlywedge\left(V_{0} \curlywedge V_{0}\right)$
5. $\left(V_{0} \curlywedge V_{3}\right) \curlywedge V_{3}$
24. $\left[V_{0} \curlywedge\left(V_{0} \curlywedge V_{3}\right)\right] \curlywedge\left(V_{0} \curlywedge V_{0}\right)$
6. $V_{3} \wedge\left(V_{0} \wedge V_{3}\right)$
25. $\left[V_{0} \curlywedge\left(V_{3}^{*} \curlywedge V_{0}\right)\right] \curlywedge\left(V_{0} \curlywedge V_{0}\right)$
7. $\left(V_{0} \curlywedge V_{3}\right) \curlywedge V_{3}^{*}$
26. $\left[\left(V_{3}^{*} \curlywedge V_{0}\right) \curlywedge V_{0}\right] \curlywedge\left(V_{0} \curlywedge V_{0}\right)$
8. $V_{3}^{*} \curlywedge\left(V_{0} \wedge V_{3}\right)$
27. $\left(V_{0} \wedge V_{0}\right) \curlywedge\left[V_{3} \curlywedge\left(V_{0} \curlywedge V_{0}\right)\right]$
9. $\left(V_{3}^{*} \curlywedge V_{0}\right) \curlywedge V_{3}$
28. $\left(V_{0} \curlywedge V_{0}\right) \curlywedge\left[\left(V_{0} \curlywedge V_{0}\right) \curlywedge V_{3}\right]$
10. $V_{3} \curlywedge\left(V_{3}^{*} \curlywedge V_{0}\right)$
29. $\left(V_{0} \wedge V_{0}\right) \curlywedge\left[V_{3}^{*} \curlywedge\left(V_{0} \wedge V_{0}\right)\right]$
11. $\left(V_{3}^{*} \curlywedge V_{0}\right) \wedge V_{3}^{*}$
30. $\left(V_{0} \wedge V_{0}\right) \wedge\left[\left(V_{0} \wedge V_{0}\right) \wedge V_{3}^{*}\right]$
12. $V_{3}^{*} \curlywedge\left(V_{3}^{*} \curlywedge V_{0}\right)$
31. $\left(V_{0} \wedge V_{0}\right) \curlywedge\left[V_{0} \curlywedge\left(V_{3} \curlywedge V_{0}\right)\right]$
13. $\left(V_{0} \curlywedge V_{3}^{*}\right) \curlywedge V_{3}$
32. $\left(V_{0} \curlywedge V_{0}\right) \curlywedge\left[\left(V_{3} \wedge V_{0}\right) \curlywedge V_{0}\right]$
14. $V_{3} \curlywedge\left(V_{0} \wedge V_{3}^{*}\right)$
33. $\left(V_{0} \curlywedge V_{0}\right) \curlywedge\left[V_{0} \curlywedge\left(V_{0} \curlywedge V_{3}\right)\right]$
15. $\left(V_{0} \curlywedge V_{3}^{*}\right) \wedge V_{3}^{*}$
34. $\left(V_{0} \wedge V_{0}\right) \wedge\left[\left(V_{0} \wedge V_{3}\right) \wedge V_{0}\right]$
16. $V_{3}^{*} \curlywedge\left(V_{0} \curlywedge V_{3}^{*}\right)$
35. $\left(V_{0} \curlywedge V_{0}\right) \curlywedge\left[V_{0} \curlywedge\left(V_{3}^{*} \curlywedge V_{0}\right)\right]$
17. $\left[V_{3} \curlywedge\left(V_{0} \curlywedge V_{0}\right)\right] \curlywedge\left(V_{0} \curlywedge V_{0}\right)$
36. $\left(V_{0} \curlywedge V_{0}\right) \wedge\left[\left(V_{3}^{*} \curlywedge V_{0}\right) \curlywedge V_{0}\right]$
18. $\left[\left(V_{0} \curlywedge V_{0}\right) \wedge V_{3}\right] \curlywedge\left(V_{0} \curlywedge V_{0}\right)$
37. $\left[\left(V_{0} \wedge V_{3}^{*}\right) \curlywedge V_{0}\right] \curlywedge\left(V_{0} \curlywedge V_{0}\right)$
19. $\left[V_{3}^{*} \curlywedge\left(V_{0} \curlywedge V_{0}\right)\right] \curlywedge\left(V_{0} \curlywedge V_{0}\right)$
38. $\left[V_{0} \curlywedge\left(V_{0} \curlywedge V_{3}^{*}\right)\right] \curlywedge\left(V_{0} \curlywedge V_{0}\right)$ 

39. $\left(V_{0} \curlywedge V_{0}\right) \curlywedge\left[\left(V_{0} \curlywedge V_{3}^{*}\right) \curlywedge V_{0}\right]$
67. $\left[\left(V_{0} \curlywedge\left(V_{3} \curlywedge V_{0}\right)\right) \curlywedge V_{0}\right] \curlywedge V_{0}$
40. $\left(V_{0} \curlywedge V_{0}\right) \curlywedge\left[V_{0} \curlywedge\left(V_{0} \curlywedge V_{3}^{*}\right)\right]$
68. $\left[\left(V_{0} \curlywedge\left(V_{0} \prec V_{3}\right)\right) \curlywedge V_{0}\right] \curlywedge V_{0}$
41. $\left(V_{3} \wedge V_{3}\right) \wedge V_{0}$
69. $\left[\left(V_{0} \curlywedge\left(V_{3}^{*} \curlywedge V_{0}\right)\right) \curlywedge V_{0}\right] \curlywedge V_{0}$
42. $\left(V_{3}^{*} \wedge V_{3}\right) \wedge V_{0}$
70. $\left[\left(V_{0} \curlywedge\left(V_{0} \curlywedge V_{3}^{*}\right)\right) \curlywedge V_{0}\right] \curlywedge V_{0}$
43. $V_{0} \curlywedge\left(V_{3} \wedge V_{3}^{*}\right)$
71. $\left[\left(\left(V_{0} \curlywedge V_{0}\right) \wedge V_{3}\right) \wedge V_{0}\right] \wedge V_{0}$
44. $\left(V_{3} \wedge V_{3}^{*}\right) \wedge V_{0}$
72. $\left[\left(\left(V_{0} \wedge V_{0}\right) \curlywedge V_{3}^{*}\right) \curlywedge V_{0}\right] \curlywedge V_{0}$
45. $V_{0} \curlywedge\left(V_{3}^{*} \curlywedge V_{3}^{*}\right)$
73. $\left[\left(\left(V_{3} \wedge V_{0}\right) \wedge V_{0}\right) \wedge V_{0}\right] \wedge V_{0}$
46. $\left(V_{3}^{*} \curlywedge V_{3}^{*}\right) \curlywedge V_{0}$
74. $\left[\left(\left(V_{0} \curlywedge V_{3}\right) \curlywedge V_{0}\right) \wedge V_{0}\right] \curlywedge V_{0}$
47. $V_{0} \wedge\left(V_{3} \wedge V_{3}\right)$
75. $\left[\left(\left(V_{3}^{*} \curlywedge V_{0}\right) \curlywedge V_{0}\right) \wedge V_{0}\right] \curlywedge V_{0}$
48. $V_{0} \curlywedge\left(V_{3}^{*} \wedge V_{3}\right)$
49. $V_{0} \curlywedge\left[\left(V_{3} \curlywedge V_{0}\right) \curlywedge\left(V_{0} \curlywedge V_{0}\right)\right]$
76. $\left[\left(\left(V_{0} \wedge V_{3}^{*}\right) \wedge V_{0}\right) \wedge V_{0}\right] \wedge V_{0}$
50. $V_{0} \curlywedge\left[\left(V_{0} \curlywedge V_{3}\right) \curlywedge\left(V_{0} \curlywedge V_{0}\right)\right]$
77. $V_{0} \curlywedge\left[\left(V_{3} \curlywedge\left(V_{0} \curlywedge V_{0}\right) \curlywedge V_{0}\right)\right]$
51. $V_{0} \curlywedge\left[\left(V_{3}^{*} \curlywedge V_{0}\right) \curlywedge\left(V_{0} \curlywedge V_{0}\right)\right]$
52. $V_{0} \curlywedge\left[\left(V_{0} \wedge V_{3}^{*}\right) \curlywedge\left(V_{0} \curlywedge V_{0}\right)\right]$
53. $V_{0} \curlywedge\left[\left(V_{0} \curlywedge V_{0}\right) \curlywedge\left(V_{3} \wedge V_{0}\right)\right]$
78. $V_{0} \curlywedge\left[\left(V_{3}^{*} \curlywedge\left(V_{0} \curlywedge V_{0}\right) \curlywedge V_{0}\right)\right]$
79. $V_{0} \curlywedge\left[\left(V_{0} \curlywedge\left(V_{3} \curlywedge V_{0}\right) \curlywedge V_{0}\right)\right]$
54. $V_{0} \curlywedge\left[\left(V_{0} \curlywedge V_{0}\right) \curlywedge\left(V_{0} \curlywedge V_{3}\right)\right]$
80. $V_{0} \curlywedge\left[\left(V_{0} \curlywedge\left(V_{0} \curlywedge V_{3}\right) \curlywedge V_{0}\right)\right]$
81. $V_{0} \curlywedge\left[\left(V_{0} \curlywedge\left(V_{3}^{*} \curlywedge V_{0}\right) \curlywedge V_{0}\right)\right]$
82. $V_{0} \wedge\left[\left(V_{0} \wedge\left(V_{0} \wedge V_{3}^{*}\right) \wedge V_{0}\right)\right]$
55. $V_{0} \curlywedge\left[\left(V_{0} \wedge V_{0}\right) \curlywedge\left(V_{3}^{*} \curlywedge V_{0}\right)\right]$
56. $V_{0} \wedge\left[\left(V_{0} \wedge V_{0}\right) \curlywedge\left(V_{0} \wedge V_{3}^{*}\right)\right]$
57. $\left[\left(V_{3} \curlywedge V_{0}\right) \curlywedge\left(V_{0} \curlywedge V_{0}\right)\right] \curlywedge V_{0}$
83. $V_{0} \curlywedge\left[\left(\left(V_{0} \curlywedge V_{0}\right) \curlywedge V_{3}\right) \curlywedge V_{0}\right]$
84. $V_{0} \curlywedge\left[\left(\left(V_{0} \wedge V_{0}\right) \wedge V_{3}^{*}\right) \wedge V_{0}\right]$
85. $V_{0} \wedge\left[\left(\left(V_{3} \wedge V_{0}\right) \wedge V_{0}\right) \wedge V_{0}\right]$
58. $\left[\left(V_{0} \curlywedge V_{3}\right) \curlywedge\left(V_{0} \curlywedge V_{0}\right)\right] \curlywedge V_{0}$
86. $V_{0} \curlywedge\left[\left(\left(V_{0} \curlywedge\left(V_{3} \curlywedge V_{0}\right)\right) \curlywedge V_{0}\right]\right.$
59. $\left[\left(V_{3}^{*} \curlywedge V_{0}\right) \curlywedge\left(V_{0} \curlywedge V_{0}\right)\right] \curlywedge V_{0}$
87. $V_{0} \wedge\left[\left(\left(V_{0} \wedge V_{3}\right) \wedge V_{0}\right) \wedge V_{0}\right]$
60. $\left[\left(V_{0} \curlywedge V_{3}^{*}\right) \curlywedge\left(V_{0} \curlywedge V_{0}\right)\right] \curlywedge V_{0}$
88. $V_{0} \curlywedge\left[\left(\left(V_{3}^{*} \curlywedge V_{0}\right) \curlywedge V_{0}\right) \curlywedge V_{0}\right]$
61. $\left[\left(V_{0} \curlywedge V_{0}\right) \curlywedge\left(V_{3} \curlywedge V_{0}\right)\right] \curlywedge V_{0}$
89. $V_{0} \curlywedge\left[\left(\left(V_{0} \wedge V_{3}^{*}\right) \curlywedge V_{0}\right) \curlywedge V_{0}\right]$
62. $\left[\left(V_{0} \wedge V_{0}\right) \curlywedge\left(V_{0} \wedge V_{3}\right)\right] \curlywedge V_{0}$
90. $\left[V_{0} \curlywedge\left(V_{3} \curlywedge\left(V_{0} \curlywedge V_{0}\right)\right)\right] \curlywedge V_{0}$
63. $\left[\left(V_{0} \curlywedge V_{0}\right) \curlywedge\left(V_{3}^{*} \curlywedge V_{0}\right)\right] \curlywedge V_{0}$
91. $\left[V_{0} \curlywedge\left(V_{3}^{*} \curlywedge\left(V_{0} \curlywedge V_{0}\right)\right)\right] \curlywedge V_{0}$
64. $\left[\left(V_{0} \wedge V_{0}\right) \curlywedge\left(V_{0} \prec V_{3}^{*}\right)\right] \curlywedge V_{0}$
92. $\left[V_{0} \curlywedge\left(V_{0} \curlywedge\left(V_{3} \curlywedge V_{0}\right)\right)\right] \curlywedge V_{0}$
65. $\left[\left(V_{3} \wedge\left(V_{0} \curlywedge V_{0}\right)\right) \wedge V_{0}\right] \wedge V_{0}$
93. $\left[V_{0} \curlywedge\left(V_{0} \curlywedge\left(V_{0} \curlywedge V_{3}\right)\right)\right] \curlywedge V_{0}$
66. $\left[\left(V_{3}^{*} \wedge\left(V_{0} \wedge V_{0}\right)\right) \wedge V_{0}\right] \wedge V_{0}$
94. $\left[V_{0} \curlywedge\left(V_{0} \curlywedge\left(V_{3}^{*} \curlywedge V_{0}\right)\right)\right] \curlywedge V_{0}$
95. $\left[V_{0} \curlywedge\left(V_{0} \curlywedge\left(V_{0} \curlywedge V_{3}^{*}\right)\right)\right] \curlywedge V_{0}$ 


$$
\begin{aligned}
& \text { 96. }\left[V_{0} \curlywedge\left(\left(V_{0} \curlywedge V_{0}\right) \curlywedge V_{3}\right)\right] \curlywedge V_{0} \\
& \text { 97. }\left[V_{0} \curlywedge\left(V_{3}^{*} \curlywedge\left(V_{0} \curlywedge V_{0}\right)\right] \curlywedge V_{0}\right. \\
& \text { 98. }\left[V_{0} \curlywedge\left(\left(V_{0} \curlywedge V_{0}\right) \curlywedge V_{3}^{*}\right)\right] \curlywedge V_{0} \\
& \text { 99. }\left[V_{0} \curlywedge\left(\left(V_{3} \curlywedge V_{0}\right) \curlywedge V_{0}\right)\right] \curlywedge V_{0} \\
& \text { 100. }\left[V_{0} \wedge\left(\left(V_{0} \wedge V_{3}\right) \wedge V_{0}\right)\right] \wedge V_{0} \\
& \text { 101. }\left[V_{0} \curlywedge\left(\left(V_{3}^{*} \curlywedge V_{0}\right) \curlywedge V_{0}\right)\right] \curlywedge V_{0} \\
& \text { 102. }\left[V_{0} \curlywedge\left(\left(V_{0} \wedge V_{3}^{*}\right) \curlywedge V_{0}\right)\right] \curlywedge V_{0} \\
& \text { 103. } V_{0} \curlywedge\left[V_{0} \curlywedge\left(V_{3} \curlywedge\left(V_{0} \curlywedge V_{0}\right)\right)\right] \\
& \text { 104. } V_{0} \curlywedge\left[V_{0} \curlywedge\left(V_{3}^{*} \curlywedge\left(V_{0} \curlywedge V_{0}\right)\right)\right] \\
& \text { 105. } V_{0} \curlywedge\left[V_{0} \curlywedge\left(V_{0} \curlywedge\left(V_{3} \curlywedge V_{0}\right)\right)\right]
\end{aligned}
$$

\section{$3.3 \mathfrak{s l}_{3}(k)$-2-módulos de $\operatorname{dim}_{k}=8$.}

Os $\mathfrak{s l}_{3}(k)$-2-módulos que agora vamos a calcular são fundamentais para a construção da 2-álgebra de Lie da forma $(17: 3,0,2,2,2,2,2,2,2)$ (ver obs. 2.8.) via as 2representações de $\mathfrak{s l}_{3}(k)$ em um $k$-espaço vetorial de dimensão 8 . Usando os $\mathfrak{s l}_{3}(k)$-2módulos de dimensão $1,2,3,4,5,6,7$ (listados nas seções 3.1.1, 3.1.2, 3.1.3, 3.2.1, 3.2.2, $3.2 .3,3.2 .4,3.2 .5)$ e suas correspendentes extensões podemos achar todos os $\mathfrak{s l}_{3}(k)-2$ módulos $U$ de dimensão 8 da forma seguinte.

Seja $E$ um $\mathfrak{s l}_{3}(k)$-2-módulos de $\operatorname{dim}_{k}(E)<8$. Então temos as seguintes possibilidades:

- Se $E$ é um $\mathfrak{s l}_{3}(k)$-2-módulos de dimensão 4 enumerado na seção 3.2.2, nós podemos construir, via extensões de módulos, $\mathfrak{s l}_{3}(k)$-2-módulos de dimensão 8 do tipo $E \wedge E$ onde $E \in\left\{V_{3} \wedge V_{0}, V_{0} \wedge V_{3}, V_{3}^{*} \curlywedge V_{0}, V_{0} \wedge V_{3}^{*}\right\}$, em total vamos ter $16 \mathfrak{s l}_{3}(k)$-2módulos $U$ de dimensão 8 desse tipo.

- Se $E$ é um $\mathfrak{s l}_{3}(k)$-2-módulos de dimensão 5 listado na seção 3.2.3, podemos construir $\mathfrak{s l}_{3}(k)$-2-módulos de dimensão 8 da forma: $E \wedge V_{3}, V_{3} \wedge E, E \wedge V_{3}^{*}, V_{3}^{*} \curlywedge E$. Como temos 12 dessos módulos $E$, então vamos ter $48 \mathfrak{s l}_{3}(k)$-2-módulos $U$ de dimensão 8 desse tipo.

- Se $E$ percorre todos os $\mathfrak{s l}_{3}(k)$-2-módulos de dimensão 6 apresentados na seção 3.2.4, podemos construir $\mathfrak{s l}_{3}(k)$-2-módulos de dimensão 8 do tipo $E \wedge V_{0}$ e $V_{0}<E$, como temos 36 dessos módulos $E$, então vamos ter $72 \mathfrak{s l}_{3}(k)$-2-módulos $U$ de dimensão 8 .

- Se $E$ é um $\mathfrak{s l}_{3}(k)$-2-módulos de dimensão 7 , construiremos $\mathfrak{s l}_{3}(k)$-2-módulos de dimensão 8 do tipo $E \wedge V_{0}$ e $V_{0} \curlywedge E$ onde $E$ percorre todos os $\mathfrak{s l}_{3}(k)$-2-módulos de dimensão 7 apresentados na seção 3.2.5; como temos 114 dessos módulos $E$, então vamos ter $228 \mathfrak{s l}_{3}(k)$-2-módulos $U$ de dimensão 8 desse tipo. 
- $\mathrm{O} \mathfrak{s l}_{3}(k)$-módulo adjunto $V^{a d}$, também é um $\mathfrak{s l}_{3}(k)$-2-módulo de dimensão 8 .

Assim, em total teremos $365 \mathfrak{s l}_{3}(k)$-2-módulos de dimensão 8, que são :
1. $U^{1}=\left(V_{3}^{*} \curlywedge V_{0}\right) \curlywedge\left(V_{3}^{*} \curlywedge V_{0}\right)$
27. $U^{27}=\left[\left(V_{0} \wedge V_{3}^{*}\right) \curlywedge V_{0}\right] \curlywedge V_{3}$
2. $U^{2}=\left(V_{3} \curlywedge V_{0}\right) \curlywedge\left(V_{3}^{*} \curlywedge V_{0}\right)$
28. $U^{28}=\left[V_{0} \wedge\left(V_{0} \wedge V_{3}^{*}\right)\right] \wedge V_{3}$
3. $U^{3}=\left(V_{0} \curlywedge V_{3}^{*}\right) \curlywedge\left(V_{3}^{*} \curlywedge V_{0}\right)$
29. $U^{29}=V_{3} \curlywedge\left[V_{3} \curlywedge\left(V_{0} \wedge V_{0}\right)\right]$
4. $U^{4}=\left(V_{0} \wedge V_{3}^{*}\right) \curlywedge\left(V_{3} \curlywedge V_{0}\right)$
30. $U^{30}=V_{3} \curlywedge\left[\left(V_{0} \curlywedge V_{0}\right) \curlywedge V_{3}\right]$
5. $U^{5}=\left(V_{3}^{*} \curlywedge V_{0}\right) \curlywedge\left(V_{3} \wedge V_{0}\right)$
31. $U^{31}=V_{3} \curlywedge\left[V_{3}^{*} \curlywedge\left(V_{0} \wedge V_{0}\right)\right]$
6. $U^{6}=\left(V_{3} \wedge V_{0}\right) \wedge\left(V_{3} \wedge V_{0}\right)$
32. $U^{32}=V_{3} \wedge\left[\left(V_{0} \wedge V_{0}\right) \wedge V_{3}^{*}\right]$
7. $U^{7}=\left(V_{0} \wedge V_{3}\right) \wedge\left(V_{3}^{*} \wedge V_{0}\right)$
33. $U^{33}=V_{3} \curlywedge\left[\left(V_{3} \curlywedge V_{0}\right) \curlywedge V_{0}\right]$
8. $U^{8}=\left(V_{0} \wedge V_{3}\right) \wedge\left(V_{3} \wedge V_{0}\right)$
34. $U^{34}=V_{3} \curlywedge\left[V_{0} \curlywedge\left(V_{3} \wedge V_{0}\right)\right]$
9. $U^{9}=\left(V_{3}^{*} \curlywedge V_{0}\right) \curlywedge\left(V_{0} \wedge V_{3}^{*}\right)$
35. $U^{35}=V_{3} \curlywedge\left[\left(V_{0} \wedge V_{3}\right) \wedge V_{0}\right]$
10. $U^{10}=\left(V_{3}^{*} \curlywedge V_{0}\right) \curlywedge\left(V_{0} \curlywedge V_{3}\right)$
36. $U^{36}=V_{3} \curlywedge\left[V_{0} \curlywedge\left(V_{0} \wedge V_{3}\right)\right]$
11. $U^{11}=\left(V_{0} \curlywedge V_{3}^{*}\right) \curlywedge\left(V_{0} \curlywedge V_{3}^{*}\right)$
37. $U^{37}=V_{3} \wedge\left[\left(V_{3}^{*} \wedge V_{0}\right) \curlywedge V_{0}\right]$
12. $U^{12}=\left(V_{0} \curlywedge V_{3}^{*}\right) \curlywedge\left(V_{0} \curlywedge V_{3}\right)$
38. $U^{38}=V_{3} \wedge\left[V_{0} \curlywedge\left(V_{3}^{*} \curlywedge V_{0}\right)\right]$
13. $U^{13}=\left(V_{3} \wedge V_{0}\right) \curlywedge\left(V_{0} \wedge V_{3}^{*}\right)$
14. $U^{14}=\left(V_{3} \curlywedge V_{0}\right) \curlywedge\left(V_{0} \curlywedge V_{3}\right)$
15. $U^{15}=\left(V_{0} \wedge V_{3}\right) \curlywedge\left(V_{0} \wedge V_{3}^{*}\right)$
16. $U^{16}=\left(V_{0} \wedge V_{3}\right) \wedge\left(V_{0} \wedge V_{3}\right)$
39. $U^{39}=V_{3} \wedge\left[\left(V_{0} \wedge V_{3}^{*}\right) \wedge V_{0}\right]$
17. $U^{17}=\left[V_{3} \curlywedge\left(V_{0} \curlywedge V_{0}\right)\right] \curlywedge V_{3}$
40. $U^{40}=V_{3} \wedge\left[V_{0} \curlywedge\left(V_{0} \wedge V_{3}^{*}\right)\right]$
41. $U^{41}=\left[V_{3} \curlywedge\left(V_{0} \wedge V_{0}\right)\right] \wedge V_{3}^{*}$
42. $U^{42}=\left[\left(V_{0} \wedge V_{0}\right) \wedge V_{3}\right] \wedge V_{3}^{*}$
18. $U^{18}=\left[\left(V_{0} \wedge V_{0}\right) \wedge V_{3}\right] \wedge V_{3}$
43. $U^{43}=\left[V_{3}^{*} \curlywedge\left(V_{0} \curlywedge V_{0}\right)\right] \curlywedge V_{3}^{*}$
19. $U^{19}=\left[V_{3}^{*} \curlywedge\left(V_{0} \wedge V_{0}\right)\right] \wedge V_{3}$
20. $U^{20}=\left[\left(V_{0} \wedge V_{0}\right) \wedge V_{3}^{*}\right] \wedge V_{3}$
21. $U^{21}=\left[\left(V_{3} \wedge V_{0}\right) \wedge V_{0}\right] \wedge V_{3}$
44. $U^{44}=\left[\left(V_{0} \wedge V_{0}\right) \wedge V_{3}^{*}\right] \wedge V_{3}^{*}$
45. $U^{45}=\left[\left(V_{3} \wedge V_{0}\right) \wedge V_{0}\right] \wedge V_{3}^{*}$
22. $U^{22}=\left[V_{0} \curlywedge\left(V_{3} \wedge V_{0}\right)\right] \curlywedge V_{3}$
46. $U^{46}=\left[V_{0} \wedge\left(V_{3} \wedge V_{0}\right)\right] \curlywedge V_{3}^{*}$
47. $U^{47}=\left[\left(V_{0} \wedge V_{3}\right) \wedge V_{0}\right] \wedge V_{3}^{*}$
23. $U^{23}=\left[\left(V_{0} \wedge V_{3}\right) \wedge V_{0}\right] \wedge V_{3}$
48. $U^{48}=\left[V_{0} \wedge\left(V_{0} \wedge V_{3}\right)\right] \wedge V_{3}^{*}$
49. $U^{49}=\left[\left(V_{3}^{*} \wedge V_{0}\right) \wedge V_{0}\right] \wedge V_{3}^{*}$
24. $U^{24}=\left[V_{0} \wedge\left(V_{0} \wedge V_{3}\right)\right] \curlywedge V_{3}$
50. $U^{50}=\left[V_{0} \wedge\left(V_{3}^{*} \curlywedge V_{0}\right)\right] \wedge V_{3}^{*}$
25. $U^{25}=\left[\left(V_{3}^{*} \curlywedge V_{0}\right) \curlywedge V_{0}\right] \wedge V_{3}$
51. $U^{51}=\left[\left(V_{0} \wedge V_{3}^{*}\right) \wedge V_{0}\right] \wedge V_{3}^{*}$
26. $U^{26}=\left[V_{0} \wedge\left(V_{3}^{*} \curlywedge V_{0}\right)\right] \curlywedge V_{3}$
52. $U^{52}=\left[V_{0} \wedge\left(V_{0} \wedge V_{3}^{*}\right)\right] \wedge V_{3}^{*}$
53. $U^{53}=V_{3}^{*} \wedge\left[V_{3} \wedge\left(V_{0} \wedge V_{0}\right)\right]$ 
54. $U^{54}=V_{3}^{*} \curlywedge\left[\left(V_{0} \curlywedge V_{0}\right) \curlywedge V_{3}\right]$

55. $U^{55}=V_{3}^{*} \curlywedge\left[V_{3}^{*} \curlywedge\left(V_{0} \curlywedge V_{0}\right)\right]$

56. $U^{56}=V_{3}^{*} \curlywedge\left[\left(V_{0} \wedge V_{0}\right) \curlywedge V_{3}^{*}\right]$

57. $U^{57}=V_{3}^{*} \curlywedge\left[\left(V_{3} \wedge V_{0}\right) \curlywedge V_{0}\right]$

58. $U^{58}=V_{3}^{*} \curlywedge\left[V_{0} \curlywedge\left(V_{3} \curlywedge V_{0}\right)\right]$

59. $U^{59}=V_{3}^{*} \curlywedge\left[\left(V_{0} \curlywedge V_{3}\right) \curlywedge V_{0}\right]$

60. $U^{60}=V_{3}^{*} \curlywedge\left[V_{0} \curlywedge\left(V_{0} \curlywedge V_{3}\right)\right]$

61. $U^{61}=V_{3}^{*} \curlywedge\left[\left(V_{3}^{*} \curlywedge V_{0}\right) \curlywedge V_{0}\right]$

62. $U^{62}=V_{3}^{*} \curlywedge\left[V_{0} \curlywedge\left(V_{3}^{*} \curlywedge V_{0}\right)\right]$

63. $U^{63}=V_{3}^{*} \curlywedge\left[\left(V_{0} \curlywedge V_{3}^{*}\right) \curlywedge V_{0}\right]$

64. $U^{64}=V_{3}^{*} \curlywedge\left[V_{0} \curlywedge\left(V_{0} \curlywedge V_{3}^{*}\right)\right]$

65. $U^{65}=\left(V_{3}^{*} \curlywedge V_{3}\right) \curlywedge\left(V_{0} \curlywedge V_{0}\right)$

66. $U^{66}=\left(V_{3} \curlywedge V_{3}\right) \curlywedge\left(V_{0} \curlywedge V_{0}\right)$

67. $U^{67}=\left(V_{3} \curlywedge V_{3}^{*}\right) \curlywedge\left(V_{0} \curlywedge V_{0}\right)$

68. $U^{68}=\left(V_{3}^{*} \curlywedge V_{3}^{*}\right) \curlywedge\left(V_{0} \curlywedge V_{0}\right)$

69. $U^{69}=\left[\left(V_{3} \wedge V_{0}\right) \curlywedge\left(V_{0} \wedge V_{0}\right)\right] \curlywedge\left(V_{0} \curlywedge V_{0}\right)$

70. $U^{70}=\left[\left(V_{0} \wedge V_{0}\right) \curlywedge\left(V_{3} \wedge V_{0}\right)\right] \curlywedge\left(V_{0} \wedge V_{0}\right)$

71. $U^{71}=\left[\left(V_{0} \wedge V_{3}\right) \curlywedge\left(V_{0} \curlywedge V_{0}\right)\right] \curlywedge\left(V_{0} \curlywedge V_{0}\right)$

72. $U^{72}=\left[\left(V_{0} \curlywedge V_{0}\right) \curlywedge\left(V_{0} \curlywedge V_{3}\right)\right] \curlywedge\left(V_{0} \curlywedge V_{0}\right)$

73. $U^{73}=\left[\left(V_{3}^{*} \curlywedge V_{0}\right) \curlywedge\left(V_{0} \wedge V_{0}\right)\right] \curlywedge\left(V_{0} \wedge V_{0}\right)$

74. $U^{74}=\left[\left(V_{0} \wedge V_{0}\right) \curlywedge\left(V_{3}^{*} \curlywedge V_{0}\right)\right] \curlywedge\left(V_{0} \curlywedge V_{0}\right)$

75. $U^{75}=\left[\left(V_{0} \wedge V_{3}^{*}\right) \curlywedge\left(V_{0} \curlywedge V_{0}\right)\right] \curlywedge\left(V_{0} \curlywedge V_{0}\right)$

76. $U^{76}=\left[\left(V_{0} \curlywedge V_{0}\right) \curlywedge\left(V_{0} \curlywedge V_{3}^{*}\right)\right] \curlywedge\left(V_{0} \curlywedge V_{0}\right)$

77. $U^{77}=\left[\left[V_{3} \curlywedge\left(V_{0} \curlywedge V_{0}\right)\right] \curlywedge V_{0}\right] \curlywedge\left(V_{0} \curlywedge V_{0}\right)$

78. $U^{78}=\left[\left[\left(V_{0} \curlywedge V_{0}\right) \curlywedge V_{3}\right] \curlywedge V_{0}\right] \curlywedge\left(V_{0} \curlywedge V_{0}\right)$

79. $U^{79}=\left[\left[V_{3}^{*} \curlywedge\left(V_{0} \curlywedge V_{0}\right)\right] \curlywedge V_{0}\right] \curlywedge\left(V_{0} \curlywedge V_{0}\right)$

80. $\left.U^{80}=\left[\left[\left(V_{0} \wedge V_{0}\right) \wedge V_{3}^{*}\right)\right] \curlywedge V_{0}\right] \curlywedge\left(V_{0} \wedge V_{0}\right)$

81. $\left.\left.U^{81}=\left[\left(V_{3} \curlywedge V_{0}\right) \curlywedge V_{0}\right)\right] \curlywedge V_{0}\right] \curlywedge\left(V_{0} \curlywedge V_{0}\right)$
82. $U^{82}=\left[\left[V_{0} \curlywedge\left(V_{3} \curlywedge V_{0}\right)\right] \curlywedge V_{0}\right] \curlywedge\left(V_{0} \curlywedge V_{0}\right)$

83. $\left.U^{83}=\left[\left[\left(V_{0} \wedge V_{3}\right) \wedge V_{0}\right)\right] \curlywedge V_{0}\right] \curlywedge\left(V_{0} \wedge V_{0}\right)$

84. $U^{84}=\left[\left[V_{0} \curlywedge\left(V_{0} \curlywedge V_{3}\right)\right] \curlywedge V_{0}\right] \curlywedge\left(V_{0} \curlywedge V_{0}\right)$

85. $U^{85}=\left[\left[\left(V_{3}^{*} \curlywedge V_{0}\right) \curlywedge V_{0}\right] \curlywedge V_{0}\right] \curlywedge\left(V_{0} \curlywedge V_{0}\right)$

86. $U^{86}=\left[\left[V_{0} \wedge\left(V_{3}^{*} \curlywedge V_{0}\right)\right] \curlywedge V_{0}\right] \curlywedge\left(V_{0} \wedge V_{0}\right)$

87. $\left.U^{87}=\left[\left[\left(V_{0} \wedge V_{3}^{*}\right) \wedge V_{0}\right)\right] \curlywedge V_{0}\right] \curlywedge\left(V_{0} \wedge V_{0}\right)$

88. $U^{88}=\left[\left[V_{0} \curlywedge\left(V_{0} \wedge V_{3}^{*}\right)\right] \curlywedge V_{0}\right] \curlywedge\left(V_{0} \curlywedge V_{0}\right)$

89. $U^{89}=\left[V_{0} \curlywedge\left[V_{3} \curlywedge\left(V_{0} \curlywedge V_{0}\right)\right]\right] \curlywedge\left(V_{0} \curlywedge V_{0}\right)$

90. $U^{90}=\left[V_{0} \curlywedge\left[\left(V_{0} \curlywedge V_{0}\right) \curlywedge V_{3}\right]\right] \curlywedge\left(V_{0} \curlywedge V_{0}\right)$

91. $U^{91}=\left[V_{0} \curlywedge\left[V_{3}^{*} \curlywedge\left(V_{0} \wedge V_{0}\right)\right]\right] \curlywedge\left(V_{0} \curlywedge V_{0}\right)$

92. $\left.U^{92}=\left[V_{0} \curlywedge\left[\left(V_{0} \curlywedge V_{0}\right) \curlywedge V_{3}^{*}\right)\right]\right] \curlywedge\left(V_{0}<V_{0}\right)$

93. $\left.U^{93}=\left[V_{0} \curlywedge\left[\left(V_{3} \wedge V_{0}\right) \curlywedge V_{0}\right)\right]\right] \curlywedge\left(V_{0}<V_{0}\right)$

94. $U^{94}=\left[V_{0} \curlywedge\left[V_{0} \curlywedge\left(V_{3} \curlywedge V_{0}\right)\right]\right] \curlywedge\left(V_{0} \curlywedge V_{0}\right)$

95. $\left.U^{95}=\left[V_{0} \curlywedge\left[\left(V_{0} \wedge V_{3}\right) \curlywedge V_{0}\right)\right]\right] \curlywedge\left(V_{0} \wedge V_{0}\right)$

96. $U^{96}=\left[V_{0} \curlywedge\left[V_{0} \curlywedge\left(V_{0} \curlywedge V_{3}\right)\right]\right] \curlywedge\left(V_{0} \curlywedge V_{0}\right)$

97. $\left.U^{97}=\left[V_{0} \wedge\left[\left(V_{3}^{*} \curlywedge V_{0}\right) \curlywedge V_{0}\right)\right]\right] \curlywedge\left(V_{0} \wedge V_{0}\right)$

98. $U^{98}=\left[V_{0} \curlywedge\left[V_{0} \curlywedge\left(V_{3}^{*} \curlywedge V_{0}\right)\right]\right] \curlywedge\left(V_{0} \curlywedge V_{0}\right)$

99. $\left.U^{99}=\left[V_{0} \curlywedge\left[\left(V_{0} \wedge V_{3}^{*}\right) \curlywedge V_{0}\right)\right]\right] \curlywedge\left(V_{0} \curlywedge V_{0}\right)$

100. $U^{100}=\left[V_{0} \wedge\left[V_{0} \curlywedge\left(V_{0} \wedge V_{3}^{*}\right)\right]\right] \curlywedge\left(V_{0} \wedge V_{0}\right)$

101. $U^{101}=\left(V_{0} \curlywedge V_{0}\right) \curlywedge\left(V_{3}^{*} \curlywedge V_{3}\right)$

102. $U^{102}=\left(V_{0} \wedge V_{0}\right) \curlywedge\left(V_{3} \curlywedge V_{3}\right)$

103. $U^{103}=\left(V_{0} \curlywedge V_{0}\right) \curlywedge\left(V_{3} \curlywedge V_{3}^{*}\right)$

104. $U^{104}=\left(V_{0} \curlywedge V_{0}\right) \curlywedge\left(V_{3}^{*} \curlywedge V_{3}^{*}\right)$

105. $U^{105}=\left(V_{0} \curlywedge V_{0}\right) \curlywedge\left[\left(V_{3} \wedge V_{0}\right) \curlywedge\left(V_{0} \curlywedge V_{0}\right)\right]$

106. $U^{106}=\left(V_{0} \wedge V_{0}\right) \curlywedge\left[\left(V_{0} \wedge V_{0}\right) \curlywedge\left(V_{3} \curlywedge V_{0}\right)\right]$

107. $U^{107}=\left(V_{0} \wedge V_{0}\right) \curlywedge\left[\left(V_{0} \wedge V_{3}\right) \curlywedge\left(V_{0} \curlywedge V_{0}\right)\right]$

108. $U^{108}=\left(V_{0} \wedge V_{0}\right) \curlywedge\left[\left(V_{0} \wedge V_{0}\right) \curlywedge\left(V_{0} \wedge V_{3}\right)\right]$

109. $U^{109}=\left(V_{0} \curlywedge V_{0}\right) \curlywedge\left[\left(V_{3}^{*} \curlywedge V_{0}\right) \curlywedge\left(V_{0} \curlywedge V_{0}\right)\right]$

110. $U^{110}=\left(V_{0} \curlywedge V_{0}\right) \curlywedge\left[\left(V_{0} \curlywedge V_{0}\right) \curlywedge\left(V_{3}^{*} \curlywedge V_{0}\right)\right]$ 
111. $U^{111}=\left(V_{0} \curlywedge V_{0}\right) \curlywedge\left[\left(V_{0} \curlywedge V_{3}^{*}\right) \curlywedge\left(V_{0} \curlywedge V_{0}\right)\right]$

112. $U^{112}=\left(V_{0} \wedge V_{0}\right) \curlywedge\left[\left(V_{0} \wedge V_{0}\right) \curlywedge\left(V_{0} \wedge V_{3}^{*}\right)\right]$

113. $U^{113}=\left(V_{0} \wedge V_{0}\right) \curlywedge\left[\left[V_{3} \wedge\left(V_{0} \wedge V_{0}\right)\right] \curlywedge V_{0}\right]$

114. $U^{114}=\left(V_{0} \wedge V_{0}\right) \curlywedge\left[\left[\left(V_{0} \wedge V_{0}\right) \wedge V_{3}\right] \wedge V_{0}\right]$

115. $U^{115}=\left(V_{0} \curlywedge V_{0}\right) \curlywedge\left[\left[V_{3}^{*} \curlywedge\left(V_{0} \curlywedge V_{0}\right)\right] \curlywedge V_{0}\right]$

116. $\left.U^{116}=\left(V_{0} \wedge V_{0}\right) \curlywedge\left[\left[\left(V_{0} \wedge V_{0}\right) \wedge V_{3}^{*}\right)\right] \curlywedge V_{0}\right]$

117. $\left.U^{117}=\left(V_{0} \curlywedge V_{0}\right) \curlywedge\left[\left[\left(V_{3} \curlywedge V_{0}\right) \curlywedge V_{0}\right)\right] \curlywedge V_{0}\right]$

118. $U^{118}=\left(V_{0} \curlywedge V_{0}\right) \curlywedge\left[\left[V_{0} \curlywedge\left(V_{3} \wedge V_{0}\right)\right] \curlywedge V_{0}\right]$

119. $\left.U^{119}=\left(V_{0} \wedge V_{0}\right) \wedge\left[\left[\left(V_{0} \wedge V_{3}\right) \wedge V_{0}\right)\right] \wedge V_{0}\right]$

120. $U^{120}=\left(V_{0} \wedge V_{0}\right) \curlywedge\left[\left[V_{0} \wedge\left(V_{0} \wedge V_{3}\right)\right] \curlywedge V_{0}\right]$

121. $U^{121}=\left(V_{0} \wedge V_{0}\right) \wedge\left[\left[\left(V_{3}^{*} \wedge V_{0}\right) \wedge V_{0}\right] \wedge V_{0}\right]$

122. $U^{122}=\left(V_{0} \curlywedge V_{0}\right) \curlywedge\left[\left[V_{0} \curlywedge\left(V_{3}^{*} \curlywedge V_{0}\right)\right] \curlywedge V_{0}\right]$

123. $\left.U^{123}=\left(V_{0} \wedge V_{0}\right) \curlywedge\left[\left[\left(V_{0} \wedge V_{3}^{*}\right) \wedge V_{0}\right)\right] \curlywedge V_{0}\right]$

124. $U^{124}=\left(V_{0} \curlywedge V_{0}\right) \curlywedge\left[\left[V_{0} \curlywedge\left(V_{0} \curlywedge V_{3}^{*}\right)\right] \curlywedge V_{0}\right]$

125. $U^{125}=\left(V_{0} \wedge V_{0}\right) \curlywedge\left[V_{0} \wedge\left[V_{3} \wedge\left(V_{0} \wedge V_{0}\right)\right]\right]$

126. $U^{126}=\left(V_{0} \wedge V_{0}\right) \curlywedge\left[V_{0} \wedge\left[\left(V_{0} \wedge V_{0}\right) \wedge V_{3}\right]\right]$

127. $U^{127}=\left(V_{0} \wedge V_{0}\right) \curlywedge\left[V_{0} \wedge\left[V_{3}^{*} \curlywedge\left(V_{0} \wedge V_{0}\right)\right]\right]$

128. $\left.U^{128}=\left(V_{0} \wedge V_{0}\right) \wedge\left[V_{0} \wedge\left[\left(V_{0} \wedge V_{0}\right) \curlywedge V_{3}^{*}\right)\right]\right]$

129. $\left.U^{129}=\left(V_{0} \wedge V_{0}\right) \curlywedge\left[V_{0} \curlywedge\left[\left(V_{3} \wedge V_{0}\right) \curlywedge V_{0}\right)\right]\right]$

130. $U^{130}=\left(V_{0} \wedge V_{0}\right) \curlywedge\left[V_{0} \wedge\left[V_{0} \wedge\left(V_{3} \wedge V_{0}\right)\right]\right]$

131. $\left.U^{131}=\left(V_{0} \wedge V_{0}\right) \curlywedge\left[V_{0} \wedge\left[\left(V_{0} \wedge V_{3}\right) \curlywedge V_{0}\right)\right]\right]$

132. $U^{132}=\left(V_{0} \wedge V_{0}\right) \curlywedge\left[V_{0} \wedge\left[V_{0} \wedge\left(V_{0} \wedge V_{3}\right)\right]\right]$

133. $\left.U^{133}=\left(V_{0} \wedge V_{0}\right) \wedge\left[V_{0} \wedge\left[\left(V_{3}^{*} \wedge V_{0}\right) \wedge V_{0}\right)\right]\right]$

134. $U^{134}=\left(V_{0} \wedge V_{0}\right) \curlywedge\left[V_{0} \curlywedge\left[V_{0} \curlywedge\left(V_{3}^{*} \curlywedge V_{0}\right)\right]\right]$

135. $\left.U^{135}=\left(V_{0} \curlywedge V_{0}\right) \curlywedge\left[V_{0} \curlywedge\left[\left(V_{0} \curlywedge V_{3}^{*}\right) \curlywedge V_{0}\right)\right]\right]$

136. $U^{136}=\left(V_{0} \wedge V_{0}\right) \curlywedge\left[V_{0} \wedge\left[V_{0} \wedge\left(V_{0} \wedge V_{3}^{*}\right)\right]\right]$

137. $U^{137}=\left[\left(V_{3} \curlywedge V_{0}\right) \curlywedge V_{3}\right] \curlywedge V_{0}$

138. $U^{138}=\left[V_{3} \curlywedge\left(V_{3} \curlywedge V_{0}\right)\right] \curlywedge V_{0}$
139. $U^{139}=\left[\left(V_{3} \curlywedge V_{0}\right) \curlywedge V_{3}^{*}\right] \curlywedge V_{0}$

140. $U^{140}=\left[V_{3}^{*} \curlywedge\left(V_{3} \curlywedge V_{0}\right)\right] \curlywedge V_{0}$

141. $U^{141}=\left[\left(V_{0} \wedge V_{3}\right) \wedge V_{3}\right] \curlywedge V_{0}$

142. $U^{142}=\left[V_{3} \wedge\left(V_{0} \wedge V_{3}\right)\right] \wedge V_{0}$

143. $U^{143}=\left[\left(V_{0} \wedge V_{3}\right) \curlywedge V_{3}^{*}\right] \curlywedge V_{0}$

144. $U^{144}=\left[V_{3}^{*} \curlywedge\left(V_{0} \curlywedge V_{3}\right)\right] \curlywedge V_{0}$

145. $U^{145}=\left[\left(V_{3}^{*} \wedge V_{0}\right) \curlywedge V_{3}\right] \wedge V_{0}$

146. $U^{146}=\left[V_{3} \curlywedge\left(V_{3}^{*} \curlywedge V_{0}\right)\right] \curlywedge V_{0}$

147. $U^{147}=\left[\left(V_{3}^{*} \curlywedge V_{0}\right) \curlywedge V_{3}^{*}\right] \curlywedge V_{0}$

148. $U^{148}=\left[V_{3}^{*} \curlywedge\left(V_{3}^{*} \curlywedge V_{0}\right)\right] \curlywedge V_{0}$

149. $U^{149}=\left[\left(V_{0} \wedge V_{3}^{*}\right) \curlywedge V_{3}\right] \curlywedge V_{0}$

150. $U^{150}=\left[V_{3} \curlywedge\left(V_{0} \curlywedge V_{3}^{*}\right)\right] \curlywedge V_{0}$

151. $U^{151}=\left[\left(V_{0} \curlywedge V_{3}^{*}\right) \curlywedge V_{3}^{*}\right] \curlywedge V_{0}$

152. $U^{152}=\left[V_{3}^{*} \curlywedge\left(V_{0} \curlywedge V_{3}^{*}\right)\right] \curlywedge V_{0}$

153. $U^{153}=\left[\left[V_{3} \curlywedge\left(V_{0} \curlywedge V_{0}\right)\right] \curlywedge\left(V_{0} \curlywedge V_{0}\right)\right] \curlywedge V_{0}$

154. $U^{154}=\left[\left[\left(V_{0} \wedge V_{0}\right) \curlywedge V_{3}\right] \curlywedge\left(V_{0} \wedge V_{0}\right)\right] \curlywedge V_{0}$

155. $U^{155}=\left[\left[V_{3}^{*} \curlywedge\left(V_{0} \curlywedge V_{0}\right)\right] \curlywedge\left(V_{0} \curlywedge V_{0}\right)\right] \curlywedge V_{0}$

156. $U^{156}=\left[\left[\left(V_{0} \wedge V_{0}\right) \wedge V_{3}^{*}\right] \curlywedge\left(V_{0} \wedge V_{0}\right)\right] \wedge V_{0}$

157. $U^{157}=\left[\left[\left(V_{3} \curlywedge V_{0}\right) \curlywedge V_{0}\right] \curlywedge\left(V_{0} \curlywedge V_{0}\right)\right] \curlywedge V_{0}$

158. $U^{158}=\left[\left[V_{0} \curlywedge\left(V_{3} \curlywedge V_{0}\right)\right] \curlywedge\left(V_{0} \curlywedge V_{0}\right)\right] \curlywedge V_{0}$

159. $U^{159}=\left[\left[\left(V_{0} \curlywedge V_{3}\right) \curlywedge V_{0}\right] \curlywedge\left(V_{0} \curlywedge V_{0}\right)\right] \curlywedge V_{0}$

160. $U^{160}=\left[\left[V_{0} \curlywedge\left(V_{0} \curlywedge V_{3}\right)\right] \curlywedge\left(V_{0} \curlywedge V_{0}\right)\right] \curlywedge V_{0}$

161. $U^{161}=\left[\left[V_{0} \curlywedge\left(V_{3}^{*} \curlywedge V_{0}\right)\right] \curlywedge\left(V_{0} \curlywedge V_{0}\right)\right] \curlywedge V_{0}$

162. $U^{162}=\left[\left[\left(V_{3}^{*} \curlywedge V_{0}\right) \curlywedge V_{0}\right] \curlywedge\left(V_{0} \wedge V_{0}\right)\right] \wedge V_{0}$

163. $U^{163}=\left[\left(V_{0} \wedge V_{0}\right) \curlywedge\left[V_{3} \wedge\left(V_{0} \wedge V_{0}\right)\right]\right] \wedge V_{0}$

164. $U^{164}=\left[\left(V_{0} \wedge V_{0}\right) \curlywedge\left[\left(V_{0} \wedge V_{0}\right) \wedge V_{3}\right]\right] \wedge V_{0}$

165. $U^{165}=\left[\left(V_{0} \wedge V_{0}\right) \curlywedge\left[V_{3}^{*} \curlywedge\left(V_{0} \wedge V_{0}\right)\right]\right] \curlywedge V_{0}$

166. $U^{166}=\left[\left(V_{0} \curlywedge V_{0}\right) \curlywedge\left[\left(V_{0} \curlywedge V_{0}\right) \curlywedge V_{3}^{*}\right]\right] \curlywedge V_{0}$

167. $U^{167}=\left[\left(V_{0} \curlywedge V_{0}\right) \curlywedge\left[V_{0} \curlywedge\left(V_{3} \curlywedge V_{0}\right)\right]\right] \curlywedge V_{0}$ 
168. $U^{168}=\left[\left(V_{0} \curlywedge V_{0}\right) \curlywedge\left[\left(V_{3} \curlywedge V_{0}\right) \curlywedge V_{0}\right]\right] \curlywedge V_{0}$ 169. $U^{169}=\left[\left(V_{0} \wedge V_{0}\right) \curlywedge\left[V_{0} \curlywedge\left(V_{0} \curlywedge V_{3}\right)\right]\right] \curlywedge V_{0}$ 170. $U^{170}=\left[\left(V_{0} \wedge V_{0}\right) \wedge\left[\left(V_{0} \wedge V_{3}\right) \wedge V_{0}\right]\right] \wedge V_{0}$ 171. $U^{171}=\left[\left(V_{0} \curlywedge V_{0}\right) \curlywedge\left[V_{0} \curlywedge\left(V_{3}^{*} \curlywedge V_{0}\right)\right]\right] \curlywedge V_{0}$ 172. $U^{172}=\left[\left(V_{0} \wedge V_{0}\right) \wedge\left[\left(V_{3}^{*} \wedge V_{0}\right) \wedge V_{0}\right]\right] \wedge V_{0}$ 173. $U^{173}=\left[\left[\left(V_{0} \wedge V_{3}^{*}\right) \curlywedge V_{0}\right] \curlywedge\left(V_{0} \wedge V_{0}\right)\right]<V_{0}$ 174. $U^{174}=\left[\left[V_{0} \curlywedge\left(V_{0} \curlywedge V_{3}^{*}\right)\right] \curlywedge\left(V_{0} \curlywedge V_{0}\right)\right] \curlywedge V_{0}$ 175. $U^{175}=\left[\left(V_{0} \wedge V_{0}\right) \wedge\left[\left(V_{0} \wedge V_{3}^{*}\right) \wedge V_{0}\right]\right] \wedge V_{0}$ 176. $U^{176}=\left[\left(V_{0} \curlywedge V_{0}\right) \curlywedge\left[V_{0} \curlywedge\left(V_{0} \curlywedge V_{3}^{*}\right)\right]\right] \curlywedge V_{0}$ 177. $U^{177}=\left[\left(V_{3} \curlywedge V_{3}\right) \curlywedge V_{0}\right] \curlywedge V_{0}$ 178. $U^{178}=\left[\left(V_{3}^{*} \curlywedge V_{3}\right) \curlywedge V_{0}\right] \curlywedge V_{0}$ 179. $U^{179}=\left[V_{0} \curlywedge\left(V_{3} \wedge V_{3}^{*}\right)\right] \curlywedge V_{0}$ 180. $U^{180}=\left[\left(V_{3} \curlywedge V_{3}^{*}\right) \curlywedge V_{0}\right] \curlywedge V_{0}$ 181. $U^{181}=\left[V_{0} \curlywedge\left(V_{3}^{*} \curlywedge V_{3}^{*}\right)\right] \curlywedge V_{0}$ 182. $U^{182}=\left[\left(V_{3}^{*} \curlywedge V_{3}^{*}\right) \curlywedge V_{0}\right] \curlywedge V_{0}$ 183. $U^{183}=\left[V_{0} \curlywedge\left(V_{3} \curlywedge V_{3}\right)\right] \curlywedge V_{0}$ 184. $U^{184}=\left[V_{0} \curlywedge\left(V_{3}^{*} \curlywedge V_{3}\right)\right] \curlywedge V_{0}$ 185. $U^{185}=\left[V_{0} \curlywedge\left[\left(V_{3} \curlywedge V_{0}\right) \curlywedge\left(V_{0} \curlywedge V_{0}\right)\right]\right] \curlywedge V_{0}$ 186. $U^{186}=\left[V_{0} \curlywedge\left[\left(V_{0} \curlywedge V_{3}\right) \curlywedge\left(V_{0} \curlywedge V_{0}\right)\right]\right] \curlywedge V_{0}$ 187. $U^{187}=\left[V_{0} \curlywedge\left[\left(V_{3}^{*} \curlywedge V_{0}\right) \curlywedge\left(V_{0} \curlywedge V_{0}\right)\right]\right] \curlywedge V_{0}$ 188. $U^{188}=\left[V_{0} \curlywedge\left[\left(V_{0} \curlywedge V_{3}^{*}\right) \curlywedge\left(V_{0} \curlywedge V_{0}\right)\right]\right] \curlywedge V_{0}$ 189. $U^{189}=\left[V_{0} \curlywedge\left[\left(V_{0} \curlywedge V_{0}\right) \curlywedge\left(V_{3} \curlywedge V_{0}\right)\right]\right] \curlywedge V_{0}$ 190. $U^{190}=\left[V_{0} \curlywedge\left[\left(V_{0} \wedge V_{0}\right) \curlywedge\left(V_{0} \curlywedge V_{3}\right)\right]\right] \curlywedge V_{0}$ 191. $U^{191}=\left[V_{0} \curlywedge\left[\left(V_{0} \curlywedge V_{0}\right) \curlywedge\left(V_{3}^{*} \curlywedge V_{0}\right)\right]\right] \curlywedge V_{0}$ 192. $U^{192}=\left[V_{0} \curlywedge\left[\left(V_{0} \curlywedge V_{0}\right) \curlywedge\left(V_{0} \curlywedge V_{3}^{*}\right)\right]\right] \curlywedge V_{0}$ 193. $U^{193}=\left[\left[\left(V_{3}<V_{0}\right) \curlywedge\left(V_{0} \wedge V_{0}\right)\right]<V_{0}\right] \curlywedge V_{0}$ 194. $U^{194}=\left[\left[\left(V_{0} \wedge V_{3}\right) \curlywedge\left(V_{0} \wedge V_{0}\right)\right] \curlywedge V_{0}\right] \curlywedge V_{0}$ 195. $U^{195}=\left[\left[\left(V_{3}^{*} \curlywedge V_{0}\right) \curlywedge\left(V_{0} \curlywedge V_{0}\right)\right] \curlywedge V_{0}\right] \curlywedge V_{0}$
196. $U^{196}=\left[\left[\left(V_{0} \curlywedge V_{3}^{*}\right) \curlywedge\left(V_{0} \curlywedge V_{0}\right)\right] \curlywedge V_{0}\right] \curlywedge V_{0}$ 197. $U^{197}=\left[\left[\left(V_{0} \wedge V_{0}\right) \curlywedge\left(V_{3} \wedge V_{0}\right)\right] \wedge V_{0}\right] \curlywedge V_{0}$ 198. $U^{198}=\left[\left[\left(V_{0} \wedge V_{0}\right) \curlywedge\left(V_{0} \wedge V_{3}\right)\right] \wedge V_{0}\right] \wedge V_{0}$ 199. $U^{199}=\left[\left[\left(V_{0} \curlywedge V_{0}\right) \curlywedge\left(V_{3}^{*} \curlywedge V_{0}\right)\right] \curlywedge V_{0}\right] \curlywedge V_{0}$ 200. $U^{200}=\left[\left[\left(V_{0} \wedge V_{0}\right) \curlywedge\left(V_{0} \wedge V_{3}^{*}\right)\right] \curlywedge V_{0}\right] \curlywedge V_{0}$ 201. $U^{201}=\left[\left[\left(V_{3} \curlywedge\left(V_{0} \wedge V_{0}\right)\right) \curlywedge V_{0}\right] \curlywedge V_{0}\right] \curlywedge V_{0}$ 202. $U^{202}=\left[\left[\left(V_{3}^{*} \curlywedge\left(V_{0} \wedge V_{0}\right)\right) \curlywedge V_{0}\right] \wedge V_{0}\right] \curlywedge V_{0}$ 203. $U^{203}=\left[\left[\left(V_{0} \curlywedge\left(V_{3} \curlywedge V_{0}\right)\right) \curlywedge V_{0}\right] \curlywedge V_{0}\right] \curlywedge V_{0}$ 204. $U^{204}=\left[\left[\left(V_{0} \curlywedge\left(V_{0} \curlywedge V_{3}\right)\right) \curlywedge V_{0}\right] \curlywedge V_{0}\right] \curlywedge V_{0}$ 205. $U^{205}=\left[\left[\left(V_{0} \curlywedge\left(V_{3}^{*} \curlywedge V_{0}\right)\right) \curlywedge V_{0}\right] \curlywedge V_{0}\right] \curlywedge V_{0}$ 206. $U^{206}=\left[\left[\left(V_{0} \curlywedge\left(V_{0} \curlywedge V_{3}^{*}\right)\right) \curlywedge V_{0}\right] \curlywedge V_{0}\right] \curlywedge V_{0}$ 207. $U^{207}=\left[\left[\left(\left(V_{0} \wedge V_{0}\right) \wedge V_{3}\right) \curlywedge V_{0}\right] \curlywedge V_{0}\right] \curlywedge V_{0}$ 208. $U^{208}=\left[\left[\left(\left(V_{0} \wedge V_{0}\right) \wedge V_{3}^{*}\right) \wedge V_{0}\right] \wedge V_{0}\right] \curlywedge V_{0}$ 209. $U^{209}=\left[\left[\left(\left(V_{3} \wedge V_{0}\right) \curlywedge V_{0}\right)<V_{0}\right] \wedge V_{0}\right] \curlywedge V_{0}$ 210. $U^{210}=\left[\left[\left(\left(V_{0} \wedge V_{3}\right) \wedge V_{0}\right) \wedge V_{0}\right] \wedge V_{0}\right] \curlywedge V_{0}$ 211. $U^{211}=\left[\left[\left(\left(V_{3}^{*} \curlywedge V_{0}\right) \curlywedge V_{0}\right) \curlywedge V_{0}\right]<V_{0}\right] \curlywedge V_{0}$ 212. $U^{212}=\left[\left[\left(\left(V_{0} \wedge V_{3}^{*}\right) \wedge V_{0}\right) \wedge V_{0}\right] \wedge V_{0}\right] \curlywedge V_{0}$ 213. $U^{213}=\left[V_{0} \curlywedge\left[\left(V_{3} \curlywedge\left(V_{0} \curlywedge V_{0}\right) \curlywedge V_{0}\right)\right]\right] \curlywedge V_{0}$ 214. $U^{214}=\left[V_{0} \curlywedge\left[\left(V_{3}^{*} \curlywedge\left(V_{0} \curlywedge V_{0}\right) \curlywedge V_{0}\right)\right]\right] \curlywedge V_{0}$ 215. $U^{215}=\left[V_{0} \curlywedge\left[\left(V_{0} \curlywedge\left(V_{3} \curlywedge V_{0}\right) \curlywedge V_{0}\right)\right]\right] \curlywedge V_{0}$ 216. $U^{216}=\left[V_{0} \curlywedge\left[\left(V_{0} \curlywedge\left(V_{0} \wedge V_{3}\right) \curlywedge V_{0}\right)\right]\right]<V_{0}$ 217. $U^{217}=\left[V_{0} \curlywedge\left[\left(V_{0} \curlywedge\left(V_{3}^{*} \curlywedge V_{0}\right) \curlywedge V_{0}\right)\right]\right] \curlywedge V_{0}$ 218. $U^{218}=\left[V_{0} \wedge\left[\left(V_{0} \wedge\left(V_{0} \wedge V_{3}^{*}\right) \curlywedge V_{0}\right)\right]\right] \curlywedge V_{0}$ 219. $U^{219}=\left[V_{0} \curlywedge\left[\left(\left(V_{0} \wedge V_{0}\right) \curlywedge V_{3}\right) \curlywedge V_{0}\right]\right]<V_{0}$ 220. $U^{220}=\left[V_{0} \wedge\left[\left(\left(V_{0} \wedge V_{0}\right) \wedge V_{3}^{*}\right) \curlywedge V_{0}\right]\right] \wedge V_{0}$ 221. $U^{221}=\left[V_{0} \wedge\left[\left(\left(V_{3} \wedge V_{0}\right) \wedge V_{0}\right) \wedge V_{0}\right]\right]<V_{0}$ 222. $U^{222}=\left[V_{0} \wedge\left[\left(\left(V_{3} \wedge V_{0}\right) \wedge V_{0}\right) \curlywedge V_{0}\right]\right] \wedge V_{0}$ 223. $U^{223}=\left[V_{0} \wedge\left[\left(\left(V_{0} \wedge V_{3}\right) \wedge V_{0}\right) \curlywedge V_{0}\right]\right] \wedge V_{0}$ 224. $U^{224}=\left[V_{0} \curlywedge\left[\left(\left(V_{3}^{*} \curlywedge V_{0}\right) \curlywedge V_{0}\right) \curlywedge V_{0}\right]\right] \curlywedge V_{0}$ 
225. $U^{225}=\left[V_{0} \curlywedge\left[\left(\left(V_{0} \curlywedge V_{3}^{*}\right) \curlywedge V_{0}\right) \curlywedge V_{0}\right]\right] \curlywedge V_{0}$ 226. $U^{226}=\left[\left[V_{0} \curlywedge\left(V_{3} \curlywedge\left(V_{0} \curlywedge V_{0}\right)\right)\right] \curlywedge V_{0}\right] \curlywedge V_{0}$ 227. $U^{227}=\left[\left[V_{0} \curlywedge\left(V_{3}^{*} \curlywedge\left(V_{0} \wedge V_{0}\right)\right)\right] \curlywedge V_{0}\right] \curlywedge V_{0}$ 228. $U^{228}=\left[\left[V_{0} \curlywedge\left(V_{0} \curlywedge\left(V_{3} \wedge V_{0}\right)\right)\right] \curlywedge V_{0}\right] \curlywedge V_{0}$ 229. $U^{229}=\left[\left[V_{0} \curlywedge\left(V_{0} \curlywedge\left(V_{0} \wedge V_{3}\right)\right)\right] \wedge V_{0}\right] \curlywedge V_{0}$ 230. $U^{230}=\left[\left[V_{0} \curlywedge\left(V_{0} \curlywedge\left(V_{3}^{*} \curlywedge V_{0}\right)\right)\right] \curlywedge V_{0}\right] \curlywedge V_{0}$ 231. $U^{231}=\left[\left[V_{0} \wedge\left(V_{0} \wedge\left(V_{0} \wedge V_{3}^{*}\right)\right)\right] \wedge V_{0}\right] \wedge V_{0}$ 232. $U^{232}=\left[\left[V_{0} \wedge\left(\left(V_{0} \wedge V_{0}\right) \wedge V_{3}\right)\right] \curlywedge V_{0}\right] \curlywedge V_{0}$ 233. $U^{233}=\left[\left[V_{0} \wedge\left(\left(V_{0} \wedge V_{0}\right) \curlywedge V_{3}\right)\right] \wedge V_{0}\right] \curlywedge V_{0}$ 234. $U^{234}=\left[\left[V_{0} \curlywedge\left(\left(V_{0} \curlywedge V_{0}\right) \curlywedge V_{3}^{*}\right)\right] \curlywedge V_{0}\right] \curlywedge V_{0}$ 235. $U^{235}=\left[\left[V_{0} \wedge\left(\left(V_{3} \wedge V_{0}\right) \wedge V_{0}\right)\right] \wedge V_{0}\right] \curlywedge V_{0}$ 236. $U^{236}=\left[\left[V_{0} \curlywedge\left(\left(V_{0} \wedge V_{3}\right) \wedge V_{0}\right)\right] \curlywedge V_{0}\right] \curlywedge V_{0}$ 237. $U^{237}=\left[\left[V_{0} \wedge\left(\left(V_{3}^{*} \curlywedge V_{0}\right) \curlywedge V_{0}\right)\right] \curlywedge V_{0}\right] \curlywedge V_{0}$ 238. $U^{238}=\left[\left[V_{0} \curlywedge\left(\left(V_{0} \wedge V_{3}^{*}\right) \curlywedge V_{0}\right)\right] \curlywedge V_{0}\right] \curlywedge V_{0}$ 239. $U^{239}=\left[V_{0} \curlywedge\left[V_{0} \curlywedge\left(V_{3} \curlywedge\left(V_{0} \curlywedge V_{0}\right)\right)\right]\right] \curlywedge V_{0}$ 240. $U^{240}=\left[V_{0} \curlywedge\left[V_{0} \curlywedge\left(V_{3}^{*} \curlywedge\left(V_{0} \curlywedge V_{0}\right)\right)\right]\right] \curlywedge V_{0}$ 241. $U^{241}=\left[V_{0} \curlywedge\left[V_{0} \curlywedge\left(V_{0} \curlywedge\left(V_{3} \curlywedge V_{0}\right)\right)\right]\right] \curlywedge V_{0}$ 242. $U^{242}=\left[V_{0} \curlywedge\left[V_{0} \curlywedge\left(V_{0} \curlywedge\left(V_{0} \curlywedge V_{3}\right)\right)\right]\right] \curlywedge V_{0}$ 243. $U^{243}=\left[V_{0} \curlywedge\left[V_{0} \curlywedge\left(V_{0} \curlywedge\left(V_{3}^{*} \curlywedge V_{0}\right)\right)\right]\right] \curlywedge V_{0}$ 244. $U^{244}=\left[V_{0} \curlywedge\left[V_{0} \curlywedge\left(V_{0} \curlywedge\left(V_{0} \curlywedge V_{3}^{*}\right)\right)\right]\right] \curlywedge V_{0}$ 245. $U^{245}=\left[V_{0} \wedge\left[V_{0} \curlywedge\left(\left(V_{0} \wedge V_{0}\right) \curlywedge V_{3}\right)\right]\right] \wedge V_{0}$ 246. $U^{246}=\left[V_{0} \curlywedge\left[V_{0} \curlywedge\left(\left(V_{0} \wedge V_{0}\right) \curlywedge V_{3}^{*}\right)\right]\right] \curlywedge V_{0}$ 247. $U^{247}=\left[V_{0} \curlywedge\left[V_{0} \curlywedge\left(\left(V_{3} \wedge V_{0}\right) \curlywedge V_{0}\right)\right]\right] \curlywedge V_{0}$ 248. $U^{248}=\left[V_{0} \curlywedge\left[V_{0} \curlywedge\left(\left(V_{0} \wedge V_{3}\right) \curlywedge V_{0}\right)\right]\right] \curlywedge V_{0}$ 249. $U^{249}=\left[V_{0} \wedge\left[V_{0} \curlywedge\left(\left(V_{3}^{*} \curlywedge V_{0}\right) \curlywedge V_{0}\right)\right]\right] \curlywedge V_{0}$ 250. $U^{250}=\left[V_{0} \wedge\left[V_{0} \curlywedge\left(\left(V_{0} \wedge V_{3}^{*}\right) \curlywedge V_{0}\right)\right]\right] \curlywedge V_{0}$ 251. $U^{251}=V_{0} \curlywedge\left[\left(V_{3} \wedge V_{0}\right) \wedge V_{3}\right]$ 252. $U^{252}=V_{0} \curlywedge\left[V_{3} \curlywedge\left(V_{3} \curlywedge V_{0}\right)\right]$
253. $U^{253}=V_{0} \curlywedge\left[\left(V_{3} \curlywedge V_{0}\right) \curlywedge V_{3}^{*}\right]$

254. $U^{254}=V_{0} \curlywedge\left[V_{3}^{*} \curlywedge\left(V_{3} \curlywedge V_{0}\right)\right]$

255. $U^{255}=V_{0} \wedge\left[\left(V_{0} \wedge V_{3}\right) \curlywedge V_{3}\right]$

256. $U^{256}=V_{0} \curlywedge\left[V_{3} \curlywedge\left(V_{0} \wedge V_{3}\right)\right]$

257. $U^{257}=V_{0} \wedge\left[\left(V_{0} \wedge V_{3}\right) \curlywedge V_{3}^{*}\right]$

258. $U^{258}=V_{0} \curlywedge\left[V_{3}^{*} \curlywedge\left(V_{0} \curlywedge V_{3}\right)\right]$

259. $U^{259}=V_{0} \wedge\left[\left(V_{3}^{*} \curlywedge V_{0}\right) \wedge V_{3}\right]$ 260. $U^{260}=V_{0} \curlywedge\left[V_{3} \curlywedge\left(V_{3}^{*} \curlywedge V_{0}\right)\right]$ 261. $U^{261}=V_{0} \curlywedge\left[\left(V_{3}^{*} \curlywedge V_{0}\right) \curlywedge V_{3}^{*}\right]$ 262. $U^{262}=V_{0} \curlywedge\left[V_{3}^{*} \curlywedge\left(V_{3}^{*} \curlywedge V_{0}\right)\right]$ 263. $U^{263}=V_{0} \curlywedge\left[\left(V_{0} \curlywedge V_{3}^{*}\right) \curlywedge V_{3}\right]$ 264. $U^{264}=V_{0} \curlywedge\left[V_{3} \curlywedge\left(V_{0} \curlywedge V_{3}^{*}\right)\right]$ 265. $U^{265}=V_{0} \curlywedge\left[\left(V_{0} \wedge V_{3}^{*}\right) \wedge V_{3}^{*}\right]$ 266. $U^{266}=V_{0} \curlywedge\left[V_{3}^{*} \curlywedge\left(V_{0} \curlywedge V_{3}^{*}\right)\right]$ 267. $U^{267}=V_{0} \curlywedge\left[\left[V_{3} \curlywedge\left(V_{0} \curlywedge V_{0}\right)\right] \curlywedge\left(V_{0} \curlywedge V_{0}\right)\right]$ 268. $U^{268}=V_{0} \curlywedge\left[\left[\left(V_{0} \wedge V_{0}\right) \wedge V_{3}\right] \curlywedge\left(V_{0} \wedge V_{0}\right)\right]$ 269. $U^{269}=V_{0} \curlywedge\left[\left[V_{3}^{*} \curlywedge\left(V_{0} \curlywedge V_{0}\right)\right] \curlywedge\left(V_{0} \curlywedge V_{0}\right)\right]$ 270. $U^{270}=V_{0} \curlywedge\left[\left[\left(V_{0} \wedge V_{0}\right) \curlywedge V_{3}^{*}\right] \curlywedge\left(V_{0} \curlywedge V_{0}\right)\right]$ 271. $U^{271}=V_{0} \curlywedge\left[\left[\left(V_{3} \wedge V_{0}\right) \curlywedge V_{0}\right] \curlywedge\left(V_{0} \wedge V_{0}\right)\right]$ 272. $U^{272}=V_{0} \curlywedge\left[\left[V_{0} \curlywedge\left(V_{3} \wedge V_{0}\right)\right] \curlywedge\left(V_{0} \curlywedge V_{0}\right)\right]$ 273. $U^{273}=V_{0} \curlywedge\left[\left[\left(V_{0} \wedge V_{3}\right) \curlywedge V_{0}\right] \curlywedge\left(V_{0} \curlywedge V_{0}\right)\right]$ 274. $U^{274}=V_{0} \curlywedge\left[\left[V_{0} \curlywedge\left(V_{0} \curlywedge V_{3}\right)\right] \curlywedge\left(V_{0} \curlywedge V_{0}\right)\right]$ 275. $U^{275}=V_{0} \curlywedge\left[\left[V_{0} \curlywedge\left(V_{3}^{*} \curlywedge V_{0}\right)\right] \curlywedge\left(V_{0} \curlywedge V_{0}\right)\right]$ 276. $U^{276}=V_{0} \curlywedge\left[\left[\left(V_{3}^{*} \curlywedge V_{0}\right) \curlywedge V_{0}\right] \curlywedge\left(V_{0} \wedge V_{0}\right)\right]$ 277. $U^{277}=V_{0} \curlywedge\left[\left(V_{0} \curlywedge V_{0}\right) \curlywedge\left[V_{3} \curlywedge\left(V_{0} \curlywedge V_{0}\right)\right]\right]$ 278. $U^{278}=V_{0} \curlywedge\left[\left(V_{0} \curlywedge V_{0}\right) \curlywedge\left[\left(V_{0} \curlywedge V_{0}\right) \curlywedge V_{3}\right]\right]$ 279. $U^{279}=V_{0} \curlywedge\left[\left(V_{0} \curlywedge V_{0}\right) \curlywedge\left[V_{3}^{*} \curlywedge\left(V_{0} \curlywedge V_{0}\right)\right]\right]$ 280. $U^{280}=V_{0} \wedge\left[\left(V_{0} \curlywedge V_{0}\right) \curlywedge\left[\left(V_{0} \curlywedge V_{0}\right) \wedge V_{3}^{*}\right]\right]$ 281. $U^{281}=V_{0} \curlywedge\left[\left(V_{0} \curlywedge V_{0}\right) \curlywedge\left[V_{0} \curlywedge\left(V_{3} \wedge V_{0}\right)\right]\right]$ 
282. $U^{282}=V_{0} \curlywedge\left[\left(V_{0} \curlywedge V_{0}\right) \curlywedge\left[\left(V_{3} \curlywedge V_{0}\right) \curlywedge V_{0}\right]\right]$ 283. $U^{283}=V_{0} \curlywedge\left[\left(V_{0} \curlywedge V_{0}\right) \curlywedge\left[V_{0} \curlywedge\left(V_{0} \curlywedge V_{3}\right)\right]\right]$ 284. $U^{284}=V_{0} \wedge\left[\left(V_{0} \wedge V_{0}\right) \curlywedge\left[\left(V_{0} \wedge V_{3}\right) \wedge V_{0}\right]\right]$ 285. $U^{285}=V_{0} \wedge\left[\left(V_{0} \wedge V_{0}\right) \curlywedge\left[V_{0} \curlywedge\left(V_{3}^{*} \curlywedge V_{0}\right)\right]\right]$ 286. $U^{286}=V_{0} \wedge\left[\left(V_{0} \wedge V_{0}\right) \wedge\left[\left(V_{3}^{*} \curlywedge V_{0}\right) \curlywedge V_{0}\right]\right]$ 287. $U^{285}=V_{0} \wedge\left[\left[\left(V_{0} \wedge V_{3}^{*}\right) \curlywedge V_{0}\right] \curlywedge\left(V_{0} \wedge V_{0}\right)\right]$ 288. $U^{286}=V_{0} \curlywedge\left[\left[V_{0} \curlywedge\left(V_{0} \curlywedge V_{3}^{*}\right)\right] \curlywedge\left(V_{0} \curlywedge V_{0}\right)\right]$ 289. $U^{287}=V_{0}<\left[\left(V_{0} \wedge V_{0}\right) \curlywedge\left[\left(V_{0} \wedge V_{3}^{*}\right) \wedge V_{0}\right]\right]$ 290. $U^{290}=V_{0} \wedge\left[\left(V_{0} \wedge V_{0}\right) \curlywedge\left[V_{0} \wedge\left(V_{0} \wedge V_{3}^{*}\right)\right]\right]$ 291. $U^{291}=V_{0} \curlywedge\left[\left(V_{3} \curlywedge V_{3}\right) \curlywedge V_{0}\right]$ 292. $U^{292}=V_{0} \curlywedge\left[\left(V_{3}^{*} \curlywedge V_{3}\right) \curlywedge V_{0}\right]$ 293. $U^{293}=V_{0} \curlywedge\left[V_{0} \curlywedge\left(V_{3} \curlywedge V_{3}^{*}\right)\right]$ 294. $U^{294}=V_{0} \curlywedge\left[\left(V_{3} \curlywedge V_{3}^{*}\right) \curlywedge V_{0}\right]$ 295. $U^{295}=V_{0} \curlywedge\left[V_{0} \curlywedge\left(V_{3}^{*} \curlywedge V_{3}^{*}\right)\right]$ 296. $U^{296}=V_{0} \curlywedge\left[\left(V_{3}^{*} \curlywedge V_{3}^{*}\right) \curlywedge V_{0}\right]$ 297. $U^{297}=V_{0} \curlywedge\left[V_{0} \curlywedge\left(V_{3} \curlywedge V_{3}\right)\right]$ 298. $U^{298}=V_{0} \curlywedge\left[V_{0} \curlywedge\left(V_{3}^{*} \curlywedge V_{3}\right)\right]$ 299. $U^{299}=V_{0} \curlywedge\left[V_{0} \curlywedge\left[\left(V_{3} \curlywedge V_{0}\right) \curlywedge\left(V_{0} \curlywedge V_{0}\right)\right]\right]$ $300 U^{300} \curlywedge\left[V_{0} \curlywedge\left[\left(V_{0} \curlywedge V_{3}\right) \curlywedge\left(V_{0} \curlywedge V_{0}\right)\right]\right]$ 301. $U^{301}=V_{0} \curlywedge\left[V_{0} \curlywedge\left[\left(V_{3}^{*} \curlywedge V_{0}\right) \curlywedge\left(V_{0} \curlywedge V_{0}\right)\right]\right]$ 302. $U^{302}=V_{0}<\left[V_{0} \curlywedge\left[\left(V_{0} \wedge V_{3}^{*}\right) \curlywedge\left(V_{0} \curlywedge V_{0}\right)\right]\right]$ 303. $U^{303}=V_{0} \curlywedge\left[V_{0} \curlywedge\left[\left(V_{0} \wedge V_{0}\right) \curlywedge\left(V_{3} \curlywedge V_{0}\right)\right]\right]$ 304. $U^{304}=V_{0} \curlywedge\left[V_{0} \curlywedge\left[\left(V_{0} \curlywedge V_{0}\right) \curlywedge\left(V_{0} \curlywedge V_{3}\right)\right]\right]$ 305. $U^{305}=V_{0} \curlywedge\left[V_{0} \curlywedge\left[\left(V_{0} \curlywedge V_{0}\right) \curlywedge\left(V_{3}^{*} \curlywedge V_{0}\right)\right]\right]$ 306. $U^{306}=V_{0} \curlywedge\left[V_{0} \curlywedge\left[\left(V_{0} \curlywedge V_{0}\right) \curlywedge\left(V_{0} \curlywedge V_{3}^{*}\right)\right]\right]$ 307. $U^{307}=V_{0} \curlywedge\left[\left[\left(V_{3} \curlywedge V_{0}\right) \curlywedge\left(V_{0} \curlywedge V_{0}\right)\right] \curlywedge V_{0}\right]$ 308. $U^{308}=V_{0} \curlywedge\left[\left[\left(V_{0} \curlywedge V_{3}\right) \curlywedge\left(V_{0} \curlywedge V_{0}\right)\right] \curlywedge V_{0}\right]$ 309. $U^{309}=V_{0} \curlywedge\left[\left[\left(V_{3}^{*} \curlywedge V_{0}\right) \curlywedge\left(V_{0} \curlywedge V_{0}\right)\right] \curlywedge V_{0}\right]$
310. $U^{310}=V_{0} \curlywedge\left[\left[\left(V_{0} \curlywedge V_{3}^{*}\right) \curlywedge\left(V_{0} \curlywedge V_{0}\right)\right] \curlywedge V_{0}\right]$ 311. $U^{311}=V_{0} \curlywedge\left[\left[\left(V_{0} \wedge V_{0}\right) \curlywedge\left(V_{3} \curlywedge V_{0}\right)\right] \curlywedge V_{0}\right]$ 312. $U^{312}=V_{0} \curlywedge\left[\left[\left(V_{0} \wedge V_{0}\right) \curlywedge\left(V_{0} \curlywedge V_{3}\right)\right] \wedge V_{0}\right]$ 313. $U^{313}=V_{0} \curlywedge\left[\left[\left(V_{0} \wedge V_{0}\right) \curlywedge\left(V_{3}^{*} \curlywedge V_{0}\right)\right] \curlywedge V_{0}\right]$ 314. $U^{314}=V_{0} \wedge\left[\left[\left(V_{0} \wedge V_{0}\right) \curlywedge\left(V_{0} \wedge V_{3}^{*}\right)\right] \curlywedge V_{0}\right]$ 315. $U^{315}=V_{0} \curlywedge\left[\left[\left(V_{3} \curlywedge\left(V_{0} \curlywedge V_{0}\right)\right) \curlywedge V_{0}\right] \curlywedge V_{0}\right]$ 316. $U^{316}=V_{0} \curlywedge\left[\left[\left(V_{3}^{*} \curlywedge\left(V_{0} \curlywedge V_{0}\right)\right) \curlywedge V_{0}\right] \curlywedge V_{0}\right]$ 317. $U^{317}=V_{0} \curlywedge\left[\left[\left(V_{0} \curlywedge\left(V_{3} \curlywedge V_{0}\right)\right) \curlywedge V_{0}\right] \curlywedge V_{0}\right]$ 318. $U^{318}=V_{0} \curlywedge\left[\left[\left(V_{0} \curlywedge\left(V_{0} \curlywedge V_{3}\right)\right) \curlywedge V_{0}\right] \curlywedge V_{0}\right]$ 319. $U^{319}=V_{0} \curlywedge\left[\left[\left(V_{0} \curlywedge\left(V_{3}^{*} \curlywedge V_{0}\right)\right) \curlywedge V_{0}\right] \curlywedge V_{0}\right]$ 320. $U^{320}=V_{0} \curlywedge\left[\left[\left(V_{0} \curlywedge\left(V_{0} \curlywedge V_{3}^{*}\right)\right) \curlywedge V_{0}\right] \curlywedge V_{0}\right]$ 321. $U^{321}=V_{0} \curlywedge\left[\left[\left(\left(V_{0} \curlywedge V_{0}\right) \curlywedge V_{3}\right) \curlywedge V_{0}\right] \curlywedge V_{0}\right]$ 322. $U^{322}=V_{0} \curlywedge\left[\left[\left(\left(V_{0} \wedge V_{0}\right) \curlywedge V_{3}^{*}\right) \curlywedge V_{0}\right] \curlywedge V_{0}\right]$ 323. $U^{323}=V_{0} \curlywedge\left[\left[\left(\left(V_{3} \wedge V_{0}\right) \curlywedge V_{0}\right) \curlywedge V_{0}\right] \curlywedge V_{0}\right]$ 324. $U^{324}=V_{0} \curlywedge\left[\left[\left(\left(V_{0} \wedge V_{3}\right) \curlywedge V_{0}\right) \curlywedge V_{0}\right] \curlywedge V_{0}\right]$ 325. $U^{325}=V_{0} \wedge\left[\left[\left(\left(V_{3}^{*} \wedge V_{0}\right) \wedge V_{0}\right) \wedge V_{0}\right]<V_{0}\right]$ 326. $U^{326}=V_{0} \curlywedge\left[\left[\left(\left(V_{0} \wedge V_{3}^{*}\right) \curlywedge V_{0}\right) \curlywedge V_{0}\right] \curlywedge V_{0}\right]$ 327. $U^{327}=V_{0} \curlywedge\left[V_{0} \curlywedge\left[\left(V_{3} \curlywedge\left(V_{0} \curlywedge V_{0}\right) \curlywedge V_{0}\right)\right]\right]$ 328. $U^{328}=V_{0} \wedge\left[V_{0} \wedge\left[\left(V_{3}^{*} \curlywedge\left(V_{0} \curlywedge V_{0}\right) \curlywedge V_{0}\right)\right]\right]$ 329. $U^{329}=V_{0} \curlywedge\left[V_{0} \curlywedge\left[\left(V_{0} \curlywedge\left(V_{3} \curlywedge V_{0}\right) \curlywedge V_{0}\right)\right]\right]$ 330. $U^{330}=V_{0} \curlywedge\left[V_{0} \curlywedge\left[\left(V_{0} \curlywedge\left(V_{0} \curlywedge V_{3}\right) \curlywedge V_{0}\right)\right]\right]$ 331. $U^{331}=V_{0} \curlywedge\left[V_{0} \curlywedge\left[\left(V_{0} \curlywedge\left(V_{3}^{*} \curlywedge V_{0}\right) \curlywedge V_{0}\right)\right]\right]$ 332. $U^{332}=V_{0} \curlywedge\left[V_{0} \curlywedge\left[\left(V_{0} \curlywedge\left(V_{0} \curlywedge V_{3}^{*}\right) \curlywedge V_{0}\right)\right]\right]$ 333. $U^{333}=V_{0} \curlywedge\left[V_{0} \curlywedge\left[\left(\left(V_{0} \wedge V_{0}\right) \curlywedge V_{3}\right) \curlywedge V_{0}\right]\right]$ 334. $U^{334}=V_{0} \curlywedge\left[V_{0} \curlywedge\left[\left(\left(V_{0} \curlywedge V_{0}\right) \curlywedge V_{3}^{*}\right) \curlywedge V_{0}\right]\right]$ 335. $U^{335}=V_{0} \wedge\left[V_{0} \wedge\left[\left(\left(V_{3} \wedge V_{0}\right) \curlywedge V_{0}\right) \curlywedge V_{0}\right]\right]$ 336. $U^{336}=V_{0} \curlywedge\left[V_{0} \curlywedge\left[\left(\left(V_{3} \wedge V_{0}\right) \curlywedge V_{0}\right) \curlywedge V_{0}\right]\right]$ 337. $U^{337}=V_{0} \curlywedge\left[V_{0} \curlywedge\left[\left(\left(V_{0} \wedge V_{3}\right) \curlywedge V_{0}\right) \curlywedge V_{0}\right]\right]$ 338. $U^{338}=V_{0} \curlywedge\left[V_{0} \curlywedge\left[\left(\left(V_{3}^{*} \curlywedge V_{0}\right) \curlywedge V_{0}\right) \curlywedge V_{0}\right]\right]$ 

339. $U^{339}=V_{0} \curlywedge\left[V_{0} \wedge\left[\left(\left(V_{0} \wedge V_{3}^{*}\right) \curlywedge V_{0}\right) \curlywedge V_{0}\right]\right]$
353. $U^{353}=V_{0} \curlywedge\left[V_{0} \curlywedge\left[V_{0} \curlywedge\left(V_{3} \curlywedge\left(V_{0} \curlywedge V_{0}\right)\right)\right]\right]$
340. $U^{340}=V_{0} \curlywedge\left[\left[V_{0} \curlywedge\left(V_{3} \curlywedge\left(V_{0} \curlywedge V_{0}\right)\right)\right] \curlywedge V_{0}\right]$
354. $U^{354}=V_{0} \wedge\left[V_{0} \curlywedge\left[V_{0} \curlywedge\left(V_{3}^{*} \curlywedge\left(V_{0} \curlywedge V_{0}\right)\right)\right]\right]$
341. $U^{341}=V_{0} \curlywedge\left[V_{0} \curlywedge\left(V_{3}^{*} \curlywedge\left(V_{0} \curlywedge V_{0}\right)\right)\right] \curlywedge V_{0}$
342. $U^{342}=V_{0} \wedge\left[\left[V_{0} \curlywedge\left(V_{0} \curlywedge\left(V_{3} \wedge V_{0}\right)\right)\right] \curlywedge V_{0}\right]$
343. $U^{343}=V_{0} \curlywedge\left[\left[V_{0} \curlywedge\left(V_{0} \curlywedge\left(V_{0} \curlywedge V_{3}\right)\right)\right] \curlywedge V_{0}\right]$
344. $U^{344}=V_{0} \curlywedge\left[\left[V_{0} \curlywedge\left(V_{0} \curlywedge\left(V_{3}^{*} \curlywedge V_{0}\right)\right)\right] \curlywedge V_{0}\right]$
345. $U^{345}=V_{0} \curlywedge\left[\left[V_{0} \curlywedge\left(V_{0} \curlywedge\left(V_{0} \curlywedge V_{3}^{*}\right)\right)\right] \curlywedge V_{0}\right]$
346. $U^{346}=V_{0} \curlywedge\left[\left[V_{0} \curlywedge\left(\left(V_{0} \wedge V_{0}\right) \curlywedge V_{3}\right)\right] \curlywedge V_{0}\right]$
347. $U^{347}=V_{0} \wedge\left[\left[V_{0} \wedge\left(\left(V_{0} \wedge V_{0}\right) \wedge V_{3}\right)\right] \wedge V_{0}\right]$
348. $U^{348}=V_{0} \curlywedge\left[\left[V_{0} \curlywedge\left(\left(V_{0} \curlywedge V_{0}\right) \curlywedge V_{3}^{*}\right)\right] \curlywedge V_{0}\right]$
349. $U^{349}=V_{0} \curlywedge\left[\left[V_{0} \curlywedge\left(\left(V_{3} \wedge V_{0}\right) \curlywedge V_{0}\right)\right] \curlywedge V_{0}\right]$
350. $U^{350}=V_{0} \wedge\left[\left[V_{0} \curlywedge\left(\left(V_{0} \wedge V_{3}\right) \wedge V_{0}\right)\right] \curlywedge V_{0}\right]$
351. $U^{351}=V_{0} \curlywedge\left[\left[V_{0} \curlywedge\left(\left(V_{3}^{*} \curlywedge V_{0}\right) \curlywedge V_{0}\right)\right] \curlywedge V_{0}\right]$
352. $U^{352}=V_{0}<\left[\left[V_{0} \curlywedge\left(\left(V_{0}<V_{3}^{*}\right) \wedge V_{0}\right)\right]<V_{0}\right]$
355. $U^{355}=V_{0} \curlywedge\left[V_{0} \curlywedge\left[V_{0} \curlywedge\left(V_{0} \curlywedge\left(V_{3} \curlywedge V_{0}\right)\right)\right]\right]$
356. $U^{356}=V_{0} \wedge\left[V_{0} \curlywedge\left[V_{0} \curlywedge\left(V_{0} \curlywedge\left(V_{0} \curlywedge V_{3}\right)\right)\right]\right]$
357. $U^{357}=V_{0} \wedge\left[V_{0} \curlywedge\left[V_{0} \curlywedge\left(V_{0} \curlywedge\left(V_{3}^{*} \curlywedge V_{0}\right)\right)\right]\right]$
358. $U^{358}=V_{0} \curlywedge\left[V_{0} \curlywedge\left[V_{0} \curlywedge\left(V_{0} \curlywedge\left(V_{0} \curlywedge V_{3}^{*}\right)\right)\right]\right]$
359. $U^{359}=V_{0} \wedge\left[V_{0} \wedge\left[V_{0} \curlywedge\left(\left(V_{0} \curlywedge V_{0}\right) \curlywedge V_{3}\right)\right]\right]$
360. $U^{360}=V_{0} \curlywedge\left[V_{0} \curlywedge\left[V_{0} \curlywedge\left(\left(V_{0} \curlywedge V_{0}\right) \curlywedge V_{3}^{*}\right)\right]\right]$
361. $U^{361}=V_{0}<\left[V_{0} \curlywedge\left[V_{0} \curlywedge\left(\left(V_{3} \wedge V_{0}\right) \curlywedge V_{0}\right)\right]\right]$
362. $U^{362}=V_{0} \wedge\left[V_{0} \wedge\left[V_{0} \curlywedge\left(\left(V_{0} \curlywedge V_{3}\right) \curlywedge V_{0}\right)\right]\right]$
363. $U^{363}=V_{0} \curlywedge\left[V_{0} \curlywedge\left[V_{0} \curlywedge\left(\left(V_{3}^{*} \curlywedge V_{0}\right) \curlywedge V_{0}\right)\right]\right]$
364. $U^{364}=V_{0}<\left[V_{0} \curlywedge\left[V_{0} \curlywedge\left(\left(V_{0} \wedge V_{3}^{*}\right) \curlywedge V_{0}\right)\right]\right]$
365. $U^{365}=V^{a d}$, o $\mathfrak{s l}_{3}(k)$-2-módulo adjunto.

\subsubsection{Tabelas de alguns $\mathfrak{s l}_{3}(k)$-2-módulos de $\operatorname{dim}_{k}=8$.}

Aquí apresentamos tabelas que mostran as $\mathfrak{s l}_{3}(k)$-ação de alguns extensões dos $\mathfrak{s l}_{3}(k)-2$ módulos achados na seção anterior e focamos no caso de aquelas extensões que cindem.

1. Tabela para o $\mathfrak{s l}_{3}(k)-2-$ módulo $U^{2}:=\left(V_{3} \curlywedge V_{0}\right) \curlywedge\left(V_{3}^{*} \curlywedge V_{0}\right)$.

Temos a sequência exata curta:

$$
0 \rightarrow V_{3}^{*} \curlywedge V_{0} \stackrel{\iota}{\rightarrow} U^{2} \stackrel{\pi}{\rightarrow} V_{3} \curlywedge V_{0} \rightarrow 0
$$

onde $V_{3}^{*}\left\langle V_{0}:=\left\langle u, u_{1}, u_{2}, u_{3}\right\rangle\right.$ é um $\mathfrak{s l}_{3}(k)$-submódulo de $U^{2}$ com $u, u_{1}, u_{2}$ e $u_{3}$ elementos de peso $(0,0),(1,0),(1,1)$ e $(0,1)$ respectivamente e,

$U^{2} /\left(V_{3}^{*} \wedge V_{0}\right) \simeq V_{3} \wedge V_{0}$. Como $V_{3} \wedge V_{0}:=\left\langle a, a_{1}, a_{2}, a_{3}\right\rangle$ existem elementos $x, x_{1}, x_{2}, x_{3} \in U^{2}$ tal que $\pi(x)=a$ e $\pi\left(x_{i}\right)=a_{i} \operatorname{com} i=1,2,3$. Suponhamos que $x, x_{1}, x_{2}$ e, $x_{3}$ são de peso $(0,0),(1,0),(1,1)$ e, $(0,1)$ respectivamente. Então argumentando da mesma forma como no caso 3.2.4.1 temos o seguinte sistema de 
24 equações com 24 incógnitas para $\delta_{i}, i=1, \ldots, 24$.

$$
\begin{cases}x \cdot e_{12}+\delta_{1} u_{3} & =0 \\ x \cdot e_{13}+\delta_{2} u_{2} & =0 \\ x . e_{23}+\delta_{3} u_{1} & =0 \\ x \cdot e_{21}+\delta_{4} u_{3} & =0 \\ x \cdot e_{31}+\delta_{5} u_{2} & =0 \\ x \cdot e_{32}+\delta_{6} u_{1} & =0 \\ x_{1} \cdot e_{12}+x_{2}+\delta_{7} u_{2} & =0 \\ x_{1} \cdot e_{13}+x_{3}+\delta_{8} u_{3} & =0 \\ x_{1} \cdot e_{23}+\beta x+\delta_{9} u & =0 \\ x_{1} \cdot e_{21}+\delta_{10} u_{2} & =0 \\ x_{1} \cdot e_{31}+\delta_{11} u_{3} & =0 \\ x_{1} \cdot e_{32}+\kappa x+\delta_{12} u & =0 \\ x_{2} \cdot e_{12}+\delta_{13} u_{1} & =0 \\ x_{2} \cdot e_{13}+\gamma x+\delta_{14} u & =0 \\ x_{2} \cdot e_{23}+x_{3}+\delta_{15} u_{3} & =0 \\ x_{2} \cdot e_{21}+x_{1}+\delta_{16} u_{1} & =0 \\ x_{2} \cdot e_{31}+\kappa x+\delta_{17} u & =0 \\ x_{2} \cdot e_{32}+\delta_{18} u_{3} & =0 \\ x_{3} \cdot e_{12}+\gamma x+\delta_{19} u & =0 \\ x_{3} \cdot e_{13}+\delta_{20} u_{1} & =0 \\ x_{3} \cdot e_{23}+\delta_{21} u_{2} & =0 \\ x_{3} \cdot e_{21}+\beta x+\delta_{22} u & =0 \\ x_{3} \cdot e_{31}+x_{1}+\delta_{23} u_{1} & =0 \\ x_{3} \cdot e_{32}+x_{2}+\delta_{24} u_{2} & =0\end{cases}
$$

Daí resulta que:

$\delta_{1}=\delta_{2}, \delta_{3}=\delta_{4}, \delta_{5}=\delta_{6}, \delta_{7}=\delta_{13}, \delta_{8}=\delta_{20}, \delta_{10}=\delta_{16}, \delta_{11}=\delta_{23}, \delta_{15}=\delta_{21}$, $\delta_{18}=\delta_{24}, \delta_{19}+\delta_{14}=\delta_{15} \lambda+\delta_{13} \pi, \delta_{9}+\delta_{22}=\delta_{23} \pi+\delta_{21} \xi, \delta_{17}+\delta_{12}=\delta_{16} \lambda+\delta_{18} \xi$, $\kappa \delta_{1}+\delta_{18}=\delta_{7}, \kappa \delta_{4}=\delta_{11}+\delta_{16}, \kappa \delta_{2}=\delta_{7}+\delta_{24}, \beta \delta_{1}=\beta \delta_{2}=\xi \delta_{2}=\pi \delta_{2}=\gamma \delta_{3}=$ $\xi \delta_{3}=\lambda \delta_{3}=\gamma \lambda_{2}=\gamma \delta_{1}=\pi \delta_{1}=\lambda \delta_{1}=\kappa \delta_{5}=0, \gamma \delta_{6}=\gamma \delta_{5}=\kappa \delta_{2}=\kappa \delta_{1}, \kappa \delta_{4}=$ $\kappa \delta_{6}, \beta \delta_{6}=\beta \delta_{5}=\lambda \delta_{4}=\kappa \delta_{3}, \lambda \delta_{3}=\delta_{6} \pi, \xi \delta_{1}=\lambda \delta_{4}, \pi \delta_{5}=\xi \delta_{2}, \pi \delta_{20}=\pi \delta_{21}$, $\xi \delta_{8}=\xi \delta_{21}$.

Sejam:

$\begin{array}{lllll}\mu_{1}:=\delta_{1}=\delta_{2} & \mu_{4}:=\delta_{7}=\delta_{13} & \mu_{7}:=\delta_{11}=\delta_{23} & \mu_{10}:=\delta_{9} & \mu_{11}:=\delta_{22} \\ \mu_{2}:=\delta_{3}=\delta_{4} & \mu_{5}:=\delta_{8}=\delta_{20} & \mu_{8}:=\delta_{18}=\delta_{24} & \mu_{12}:=\delta_{19} & \mu_{15}:=\delta_{12} \\ \mu_{3}:=\delta_{5}=\delta_{6} & \mu_{6}:=\delta_{10}=\delta_{16} & \mu_{9}:=\delta_{21}=\delta_{15} & \mu_{13}:=\delta_{14} & \mu_{14}:=\delta_{17}\end{array}$

onde:

$\mu_{12}+\mu_{14}=\mu_{9} \lambda+\mu_{4} \pi ; \mu_{10}+\mu_{11}=\mu_{7} \pi+\mu_{9} \xi ; \mu_{14}+\mu_{15}=\mu_{6} \lambda+\mu_{8} \xi$ $\beta \mu_{1}=\xi \mu_{1}=\pi \mu_{1}=\gamma \mu_{2}=\xi \mu_{2}=\lambda \mu_{2}=\gamma \mu_{1}=\pi \mu_{1}=\lambda \mu_{1}=\kappa \mu_{3}=\kappa \mu_{2}=\beta \mu_{3}$ 
$\gamma \mu_{3}=\kappa \mu_{1} ; \kappa \mu_{2}=\kappa \mu_{3} ; \lambda \mu_{2}=\mu_{3} \pi ; \xi \mu_{1}=\lambda \mu_{2} ; \pi \mu_{3}=\xi \mu_{1} ; \pi \mu_{5}=\pi \mu_{9} ; \xi \mu_{5}=\xi \mu_{9}$.

Seja $\left\{u, u_{1}, u_{2}, u_{3}, x, x_{1}, x_{2}, x_{3}\right\}$ base de $U^{2}$, logo a ação de $\mathfrak{s l}_{3}(k)$ sobre esta base é dada na tabela seguinte:

\begin{tabular}{|c||c|c|c|c|c|c|c|c|}
\hline & $h_{1}$ & $h_{2}$ & $e_{12}$ & $e_{13}$ & $e_{23}$ & $e_{21}$ & $e_{31}$ & $e_{32}$ \\
\hline \hline$u$ & 0 & 0 & 0 & 0 & 0 & 0 & 0 & 0 \\
\hline$u_{1}$ & $u_{1}$ & 0 & 0 & 0 & $\pi u$ & $u_{2}$ & $u_{3}$ & $\lambda u$ \\
\hline$u_{2}$ & $u_{2}$ & $u_{2}$ & $u_{1}$ & $\pi u$ & 0 & 0 & $\xi u$ & $u_{3}$ \\
\hline$u_{3}$ & 0 & $u_{3}$ & $\lambda u$ & $u_{1}$ & $u_{2}$ & $\xi u$ & 0 & 0 \\
\hline$x$ & 0 & 0 & $\mu_{1} u_{3}$ & $\mu_{1} u_{2}$ & $\mu_{2} u_{1}$ & $\mu_{2} u_{3}$ & $\mu_{3} u_{2}$ & $\mu_{3} u_{1}$ \\
\hline$x_{1}$ & $x_{1}$ & 0 & $x_{2}+\mu_{4} u_{2}$ & $x_{3}+\mu_{5} u_{3}$ & $\beta x+\mu_{10} u$ & $\mu_{6} u_{2}$ & $\mu_{7} u_{3}$ & $\kappa x+\mu_{15} u$ \\
\hline$x_{2}$ & $x_{2}$ & $x_{2}$ & $\mu_{4} u_{1}$ & $\gamma x+\mu_{13} u$ & $x_{3}+\mu_{9} u_{3}$ & $x_{1}+\mu_{6} u_{1}$ & $k x+\mu_{14} u$ & $\mu_{8} u_{3}$ \\
\hline$x_{3}$ & 0 & $x_{3}$ & $\gamma x+\mu_{12} u$ & $\mu_{5} u_{1}$ & $\mu_{9} u_{2}$ & $\beta x+\mu_{11} u$ & $x_{1}+\mu_{7} u_{1}$ & $x_{2}+\mu_{8} u_{2}$ \\
\hline
\end{tabular}

Tabela 3.13: $O \mathfrak{s l}_{3}(k)-2$-módulo $U^{2}:=\left(V_{3} \curlywedge V_{0}\right) \curlywedge\left(V_{3}^{*} \curlywedge V_{0}\right)$.

Agora, se na tabela anterior fazemos $\mu_{i}=0$ para todo $i=1, \ldots, 15$, então a ação de $\mathfrak{s l}_{3}(k)$ sobre $U^{2}$ fica como na tabela 3.14 de baixo. Dessa tabela tem-se que:

$$
U^{2} \text { cinde } \Longleftrightarrow \mu_{i}=0 \forall i=1, \ldots, 15 \text { e } U^{2} \simeq\left(V_{3}^{*} \wedge V_{0}\right) \oplus\left(V_{3} \wedge V_{0}\right)
$$

\begin{tabular}{|c||c|c|c|c|c|c|c|c|}
\hline & $h_{1}$ & $h_{2}$ & $e_{12}$ & $e_{13}$ & $e_{23}$ & $e_{21}$ & $e_{31}$ & $e_{32}$ \\
\hline \hline$u$ & 0 & 0 & 0 & 0 & 0 & 0 & 0 & 0 \\
\hline$u_{1}$ & $u_{1}$ & 0 & 0 & 0 & $\pi u$ & $u_{2}$ & $u_{3}$ & $\lambda u$ \\
\hline$u_{2}$ & $u_{2}$ & $u_{2}$ & $u_{1}$ & $\pi u$ & 0 & 0 & $\xi u$ & $u_{3}$ \\
\hline$u_{3}$ & 0 & $u_{3}$ & $\lambda u$ & $u_{1}$ & $u_{2}$ & $\xi u$ & 0 & 0 \\
\hline$x$ & 0 & 0 & 0 & 0 & 0 & 0 & 0 & 0 \\
\hline$x_{1}$ & $x_{1}$ & 0 & $x_{2}$ & $x_{3}$ & $\beta x$ & 0 & 0 & $\kappa x$ \\
\hline$x_{2}$ & $x_{2}$ & $x_{2}$ & 0 & $\gamma x$ & $x_{3}$ & $x_{1}$ & $k x$ & 0 \\
\hline$x_{3}$ & 0 & $x_{3}$ & $\gamma x$ & 0 & 0 & $\beta x$ & $x_{1}$ & $x_{2}$ \\
\hline
\end{tabular}

Tabela 3.14: $O \mathfrak{s l}_{3}(k)$-2-módulo $U^{2} \simeq\left(V_{3} \wedge V_{0}\right) \oplus\left(V_{3}^{*} \curlywedge V_{0}\right)$. 
As tabelas dadas a continuação são feitas da mesma forma como em 3.3.1.1. Nós estamos interessados em achar condições necessárias e suficientes para que tais módulos cindam.

2. Tabela para o $\mathfrak{s l}_{3}(k)-2$-módulo $U^{4}:=\left(V_{0} \curlywedge V_{3}^{*}\right) \curlywedge\left(V_{3} \curlywedge V_{0}\right)$.

Seja $U^{4}:=\left\langle a, a_{1}, a_{2}, a_{3}, z, z_{1}, z_{2}, z_{3}\right\rangle$ onde a ação de $\mathfrak{s l}_{3}(k)$ sobre a base $\left\{a, a_{1}, a_{2}, a_{3}, z, z_{1}, z_{2}, z_{3}\right\} U^{4}$ é dada na tabela seguinte:

\begin{tabular}{|c||c|c|c|c|c|c|c|c|}
\hline & $h_{1}$ & $h_{2}$ & $e_{12}$ & $e_{13}$ & $e_{23}$ & $e_{21}$ & $e_{31}$ & $e_{32}$ \\
\hline \hline$a$ & 0 & 0 & 0 & 0 & 0 & 0 & 0 & 0 \\
\hline$a_{1}$ & $a_{1}$ & 0 & $a_{2}$ & $a_{3}$ & $\beta a$ & 0 & 0 & $\kappa a$ \\
\hline$a_{2}$ & $a_{2}$ & $a_{2}$ & 0 & $\gamma a$ & $a_{3}$ & $a_{1}$ & $\kappa a$ & 0 \\
\hline$a_{3}$ & 0 & $a_{3}$ & $\gamma a$ & 0 & 0 & $\beta a$ & $a_{1}$ & $a_{2}$ \\
\hline$z$ & 0 & 0 & $\delta z_{3}+\delta_{1} a_{3}$ & $\delta z_{2}+\delta_{2} a_{2}$ & $\alpha z_{1}+\delta_{3} a_{1}$ & $\alpha z_{3}+\delta_{4} a_{3}$ & $\zeta z_{2}+\delta_{5} a_{2}$ & $\zeta z_{1}+\delta_{6} a_{1}$ \\
\hline$z_{1}$ & $z_{1}$ & 0 & $\delta_{7} a_{2}$ & $\delta_{8} a_{3}$ & $\delta_{9} a$ & $z_{2}+\delta_{10} a_{2}$ & $z_{3}+\delta_{11} a_{3}$ & $\delta_{12} a$ \\
\hline$z_{2}$ & $z_{2}$ & $z_{2}$ & $z_{1}+\delta_{13} a_{1}$ & $\delta_{14} a$ & $\delta_{15} a_{3}$ & $\delta_{16} a_{1}$ & $\delta_{17} a$ & $z_{3}+\delta_{18} a_{3}$ \\
\hline$z_{3}$ & 0 & $z_{3}$ & $\delta_{19} a$ & $z_{1}+\delta_{20} a_{1}$ & $z_{2}+\delta_{21} a_{2}$ & $\delta_{22} a$ & $\delta_{23} a_{1}$ & $\delta_{24} a_{2}$ \\
\hline
\end{tabular}

Tabela 3.15: $O \mathfrak{s l}_{3}(k)-2$-módulo $U^{4}:=\left(V_{0} \wedge V_{3}^{*}\right) \curlywedge\left(V_{3} \wedge V_{0}\right)$.

Dessa tabela podemos ver que: $U^{4} \simeq\left(V_{0} \wedge V_{3}^{*}\right) \oplus\left(V_{3} \wedge V_{0}\right) \Longleftrightarrow \delta_{i}=0$ para todo $i=1, \ldots, 24$. Além disso, por 3.2.2.4 temos que $V_{0} \wedge V_{3}^{*}$ cinde $\Longleftrightarrow \delta=\alpha=\zeta=0$. Portanto:

$$
U^{4} \simeq\left(V_{0} \oplus V_{3}^{*}\right) \oplus\left(V_{3} \curlywedge V_{0}\right) \Longleftrightarrow \delta_{i}=0=\delta=\alpha=\zeta \forall i=1, \ldots, 24 .
$$

Ver tabela 3.16, abaixo.

\begin{tabular}{|c||c|c|c|c|c|c|c|c|}
\hline & $h_{1}$ & $h_{2}$ & $e_{12}$ & $e_{13}$ & $e_{23}$ & $e_{21}$ & $e_{31}$ & $e_{32}$ \\
\hline \hline$a$ & 0 & 0 & 0 & 0 & 0 & 0 & 0 & 0 \\
\hline$a_{1}$ & $a_{1}$ & 0 & $a_{2}$ & $a_{3}$ & $\beta a$ & 0 & 0 & $\kappa a$ \\
\hline$a_{2}$ & $a_{2}$ & $a_{2}$ & 0 & $\gamma a$ & $a_{3}$ & $a_{1}$ & $\kappa a$ & 0 \\
\hline$a_{3}$ & 0 & $a_{3}$ & $\gamma a$ & 0 & 0 & $\beta a$ & $a_{1}$ & $a_{2}$ \\
\hline$z$ & 0 & 0 & 0 & 0 & 0 & 0 & 0 & 0 \\
\hline$z_{1}$ & $z_{1}$ & 0 & 0 & 0 & 0 & $z_{2}$ & $z_{3}$ & 0 \\
\hline$z_{2}$ & $z_{2}$ & $z_{2}$ & $z_{1}$ & 0 & 0 & 0 & 0 & $z_{3}$ \\
\hline$z_{3}$ & 0 & $z_{3}$ & 0 & $z_{1}$ & $z_{2}$ & 0 & 0 & 0 \\
\hline
\end{tabular}

Tabela 3.16: $O \mathfrak{s l}_{3}(k)$-2-módulo $U^{4} \simeq\left(V_{0} \oplus V_{3}^{*}\right) \oplus\left(V_{3} \curlywedge V_{0}\right)$. 
3. Tabela para o $\mathfrak{s l}_{3}(k)-2$-módulo $U^{5}:=\left(V_{3}^{*} \curlywedge V_{0}\right) \curlywedge\left(V_{3} \curlywedge V_{0}\right)$.

Seja $U^{5}:=\left\langle a, a_{1}, a_{2}, a_{3}, d, d_{1}, d_{2}, d_{3}\right\rangle$, logo a ação de $\mathfrak{s l}_{3}(k)$ sobre os elementos da base $\left\{a, a_{1}, a_{2}, a_{3}, d, d_{1}, d_{2}, d_{3}\right\}$ de $U^{5}$ é dada na tabela seguinte:

\begin{tabular}{|c||c|c|c|c|c|c|c|c|}
\hline & $h_{1}$ & $h_{2}$ & $e_{12}$ & $e_{13}$ & $e_{23}$ & $e_{21}$ & $e_{31}$ & $e_{32}$ \\
\hline \hline$a$ & 0 & 0 & 0 & 0 & 0 & 0 & 0 & 0 \\
\hline$a_{1}$ & $a_{1}$ & 0 & $a_{2}$ & $a_{3}$ & $\beta a$ & 0 & 0 & $\kappa a$ \\
\hline$a_{2}$ & $a_{2}$ & $a_{2}$ & 0 & $\gamma a$ & $a_{3}$ & $a_{1}$ & $\kappa a$ & 0 \\
\hline$a_{3}$ & 0 & $a_{3}$ & $\gamma a$ & 0 & 0 & $\beta a$ & $a_{1}$ & $a_{2}$ \\
\hline$d$ & 0 & 0 & $\delta_{1} a_{3}$ & $\delta_{2} a_{2}$ & $\delta_{3} a_{1}$ & $\delta_{4} a_{3}$ & $\delta_{5} a_{2}$ & $\delta_{6} a_{1}$ \\
\hline$d_{1}$ & $d_{1}$ & 0 & $\delta_{7} a_{2}$ & $\delta_{8} a_{3}$ & $\pi d+\delta_{9} a$ & $d_{2}+\delta_{10} a_{2}$ & $d_{3}+\delta_{11} a_{3}$ & $\lambda d+\delta_{12} a$ \\
\hline$d_{2}$ & $d_{2}$ & $d_{2}$ & $d_{1}+\delta_{13} a_{1}$ & $\pi d+\delta_{14} a$ & $\delta_{15} a_{3}$ & $\delta_{16} a_{1}$ & $\xi d+\delta_{17} a$ & $d_{3}+\delta_{18} a_{3}$ \\
\hline$d_{3}$ & 0 & $d_{3}$ & $\lambda d+\delta_{19} a$ & $d_{1}+\delta_{20} a_{1}$ & $d_{2}+\delta_{21} a_{2}$ & $\xi d+\delta_{22} a$ & $\delta_{23} a_{1}$ & $\delta_{24} a_{2}$ \\
\hline
\end{tabular}

Tabela 3.17: $O \mathfrak{s l}_{3}(k)-2$-módulo $U^{5}:=\left(V_{3}^{*} \curlywedge V_{0}\right) \curlywedge\left(V_{3} \curlywedge V_{0}\right)$.

Desta tabela temos:

$$
U^{5} \simeq\left(V_{3}^{*} \curlywedge V_{0}\right) \oplus\left(V_{3} \curlywedge V_{0}\right) \Longleftrightarrow \delta_{i}=0 \quad \forall i=1, \ldots, 24 .
$$


4. Tabela para o $\mathfrak{s l}_{3}(k)-2-$ módulo $U^{7}:=\left(V_{0} \wedge V_{3}\right) \curlywedge\left(V_{3}^{*} \curlywedge V_{0}\right)$.

Seja $\left\{u, u_{1}, u_{2}, u_{3}, y, y_{1}, y_{2}, y_{3}\right\}$ uma base de $U^{7}$, a ação de $\mathfrak{s l}_{3}(k)$ sobre $U^{7}$ é dada pela tabela a seguir:

\begin{tabular}{|c||c|c|c|c|c|c|c|c|}
\hline & $h_{1}$ & $h_{2}$ & $e_{12}$ & $e_{13}$ & $e_{23}$ & $e_{21}$ & $e_{31}$ & $e_{32}$ \\
\hline \hline$u$ & 0 & 0 & 0 & 0 & 0 & 0 & 0 & 0 \\
\hline$u_{1}$ & $u_{1}$ & 0 & 0 & 0 & $\pi u$ & $u_{2}$ & $u_{3}$ & $\lambda u$ \\
\hline$u_{2}$ & $u_{2}$ & $u_{2}$ & $u_{1}$ & $\pi u$ & 0 & 0 & $\xi u$ & $u_{3}$ \\
\hline$u_{3}$ & 0 & $u_{3}$ & $\lambda u$ & $u_{1}$ & $u_{2}$ & $\xi u$ & 0 & 0 \\
\hline$y$ & 0 & 0 & $\omega y_{3}+\delta_{1} u_{3}$ & $\theta y_{2}+\delta_{2} u_{2}$ & $\theta y_{1}+\delta_{3} u_{1}$ & $\sigma y_{3}+\delta_{4} u_{3}$ & $\sigma y_{2}+\delta_{5} u_{2}$ & $\omega y_{1}+\delta_{6} u_{1}$ \\
\hline$y_{1}$ & $y_{1}$ & 0 & $y_{2}+\delta_{7} u_{2}$ & $y_{3}+\delta_{8} u_{3}$ & $\delta_{9} u$ & $\delta_{10} u_{2}$ & $\delta_{11} u_{3}$ & $\delta_{12} u$ \\
\hline$y_{2}$ & $y_{2}$ & $y_{2}$ & $\delta_{13} u_{1}$ & $\delta_{14} u$ & $y_{3}+\delta_{15} u_{3}$ & $y_{1}+\delta_{16} u_{1}$ & $\delta_{17} u$ & $\delta_{18} u_{3}$ \\
\hline$y_{3}$ & 0 & $y_{3}$ & $\delta_{19} u$ & $\delta_{20} u_{1}$ & $\delta_{21} u_{2}$ & $\delta_{22} u$ & $y_{1}+\delta_{23} u_{1}$ & $y_{2}+\delta_{24} u_{2}$ \\
\hline
\end{tabular}

Tabela 3.18: $O \mathfrak{s l}_{3}(k)-2$-módulo $U^{7}:=\left(V_{0} \curlywedge V_{3}\right) \curlywedge\left(V_{3}^{*} \curlywedge V_{0}\right)$.

Dessa tabela, podemos inferir que $U^{7}$ cinde se, e só se, $\delta_{i}=0$ para $i=1,2, \ldots, 24$, isto é, $U^{7} \simeq\left(V_{0} \wedge V_{3}\right) \oplus\left(V_{3}^{*} \wedge V_{0}\right) \Longleftrightarrow \delta_{i}=0$ para $i=1, \ldots, 24$ e, também de 3.2.2.2 temos que $V_{0} \wedge V_{3}$ cinde $\Longleftrightarrow \omega=\theta=\sigma=0$. Portanto,

$$
U^{7} \simeq\left(V_{0} \oplus V_{3}\right) \oplus\left(V_{3}^{*} \curlywedge V_{0}\right) \Longleftrightarrow \delta_{i}=0=\omega=\sigma=\theta \text { para } i=1, \ldots, 24 .
$$

5. Tabela para o $\mathfrak{s l}_{3}(k)-2$-módulo $U^{10}:=\left(V_{3}^{*} \wedge V_{0}\right) \wedge\left(V_{0} \wedge V_{3}\right)$.

Seja $U^{10}:=\left\langle v, v_{1}, v_{2}, v_{3}, b, b_{1}, b_{2}, b_{3}\right\rangle$ onde a ação de $\mathfrak{s l}_{3}(k)$ sobre a base $\left\{v, v_{1}, v_{2}, v_{3}, b, b_{1}, b_{2}, b_{3}\right\}$ de $U^{10}$ é dada na tabela seguinte:

\begin{tabular}{|c||c|c|c|c|c|c|c|c|}
\hline & $h_{1}$ & $h_{2}$ & $e_{12}$ & $e_{13}$ & $e_{23}$ & $e_{21}$ & $e_{31}$ & $e_{32}$ \\
\hline \hline$v$ & 0 & 0 & $\omega v_{3}$ & $\theta v_{2}$ & $\theta v_{1}$ & $\sigma v_{3}$ & $\sigma v_{2}$ & $\omega v_{1}$ \\
\hline$v_{1}$ & $v_{1}$ & 0 & $v_{2}$ & $v_{3}$ & 0 & 0 & 0 & 0 \\
\hline$v_{2}$ & $v_{2}$ & $v_{2}$ & 0 & 0 & $v_{3}$ & $v_{1}$ & 0 & 0 \\
\hline$v_{3}$ & 0 & $v_{3}$ & 0 & 0 & 0 & 0 & $v_{1}$ & $v_{2}$ \\
\hline$b$ & 0 & 0 & $\epsilon_{2} v_{3}$ & $\epsilon_{1} v_{2}$ & $\epsilon_{1} v_{1}$ & $\epsilon_{3} v_{3}$ & $\epsilon_{3} v_{2}$ & $\epsilon_{2} v_{1}$ \\
\hline$b_{1}$ & $b_{1}$ & 0 & $\epsilon_{4} v_{2}$ & $\epsilon_{5} v_{3}$ & $\pi b+\delta_{9} v$ & $b_{2}+\epsilon_{6} v_{2}$ & $b_{3}+\epsilon_{7} v_{3}$ & $\lambda b+\delta_{12} v$ \\
\hline$b_{2}$ & $b_{2}$ & $b_{2}$ & $b_{1}+\epsilon_{4} v_{1}$ & $\pi b+\delta_{14} v$ & $\epsilon_{8} v_{3}$ & $\epsilon_{6} v_{1}$ & $\xi b+\delta_{17} v$ & $b_{3}+\epsilon_{9} v_{3}$ \\
\hline$b_{3}$ & 0 & $b_{3}$ & $\lambda b+\delta_{19} v$ & $b_{1}+\epsilon_{5} v_{1}$ & $b_{2}+\epsilon_{8} v_{2}$ & $\xi b+\delta_{22} v$ & $\epsilon_{7} v_{1}$ & $\epsilon_{9} v_{2}$ \\
\hline
\end{tabular}

Tabela 3.19: $O \mathfrak{s l}_{3}(k)-2$-módulo $U^{10}:=\left(V_{3}^{*} \curlywedge V_{0}\right) \curlywedge\left(V_{0} \curlywedge V_{3}\right)$.

Como no caso anterior temos que $U^{10}$ cinde se, e só se, $\epsilon_{i}=0$ para todo $i=1$, $2, \ldots, 15$, isto é:

$$
U^{10} \simeq\left(V_{3}^{*} \curlywedge V_{0}\right) \oplus\left(V_{0} \curlywedge V_{3}\right) \Longleftrightarrow \epsilon_{i}=0
$$


Além disso, por 3.2.2.2 sabemos que $\left(V_{0} \curlywedge V_{3}\right) \simeq V_{0} \oplus V_{3}$ se, e só se, $\omega=\sigma=\theta=0$. Portanto

$$
U^{10} \simeq\left(V_{3}^{*}<V_{0}\right) \oplus\left(V_{0} \oplus V_{3}\right) \Longleftrightarrow \epsilon_{i}=0=\omega=\theta=\sigma, \quad \forall i=1,2, \ldots, 15
$$

nesse caso a ação de $\mathfrak{s l}_{3}(k)$ sobre $U^{10}$ cindido fica como na tabela seguinte.

\begin{tabular}{|c||c|c|c|c|c|c|c|c|}
\hline & $h_{1}$ & $h_{2}$ & $e_{12}$ & $e_{13}$ & $e_{23}$ & $e_{21}$ & $e_{31}$ & $e_{32}$ \\
\hline \hline$v$ & 0 & 0 & 0 & 0 & 0 & 0 & 0 & 0 \\
\hline$v_{1}$ & $v_{1}$ & 0 & $v_{2}$ & $v_{3}$ & 0 & 0 & 0 & 0 \\
\hline$v_{2}$ & $v_{2}$ & $v_{2}$ & 0 & 0 & $v_{3}$ & $v_{1}$ & 0 & 0 \\
\hline$v_{3}$ & 0 & $v_{3}$ & 0 & 0 & 0 & 0 & $v_{1}$ & $v_{2}$ \\
\hline$b$ & 0 & 0 & 0 & 0 & 0 & 0 & 0 & 0 \\
\hline$b_{1}$ & $b_{1}$ & 0 & 0 & 0 & $\pi b$ & $b_{2}$ & $b_{3}$ & $\lambda b$ \\
\hline$b_{2}$ & $b_{2}$ & $b_{2}$ & $b_{1}$ & $\pi b$ & 0 & 0 & $\xi b$ & $b_{3}$ \\
\hline$b_{3}$ & 0 & $b_{3}$ & $\lambda b$ & $b_{1}$ & $b_{2}$ & $\xi b$ & 0 & 0 \\
\hline
\end{tabular}

Tabela 3.20: $O \mathfrak{s l}_{3}(k)-2$-módulo $U^{10}:=\left(V_{3}^{*} \curlywedge V_{0}\right) \oplus\left(V_{0} \oplus V_{3}\right)$.

6. Tabela para o $\mathfrak{s l}_{3}(k)-2-$ módulo $U^{12}:=\left(V_{0} \wedge V_{3}^{*}\right) \curlywedge\left(V_{0} \curlywedge V_{3}\right)$.

Seja $U^{12}:=\left\langle v, v_{1}, v_{2}, v_{3}, k, k_{1}, k_{2}, k_{3}\right\rangle$ com base $\left\{v, v_{1}, v_{2}, v_{3}, k, k_{1}, k_{2}, k_{3}\right\}$; a ação de $\mathfrak{s l}_{3}(k)$ sobre essa base é dada pela tabela seguinte:

\begin{tabular}{|c||c|c|c|c|c|c|c|c|}
\hline & $h_{1}$ & $h_{2}$ & $e_{12}$ & $e_{13}$ & $e_{23}$ & $e_{21}$ & $e_{31}$ & $e_{32}$ \\
\hline \hline$v$ & 0 & 0 & $\omega v_{3}$ & $\theta v_{2}$ & $\theta v_{1}$ & $\sigma v_{3}$ & $\sigma v_{2}$ & $\omega v_{1}$ \\
\hline$v_{1}$ & $v_{1}$ & 0 & $v_{2}$ & $v_{3}$ & 0 & 0 & 0 & 0 \\
\hline$v_{2}$ & $v_{2}$ & $v_{2}$ & 0 & 0 & $v_{3}$ & $v_{1}$ & 0 & 0 \\
\hline$v_{3}$ & 0 & $v_{3}$ & 0 & 0 & 0 & 0 & $v_{1}$ & $v_{2}$ \\
\hline$k$ & 0 & 0 & $\delta k_{3}+\delta_{1} v_{3}$ & $\delta k_{2}+\delta_{2} v_{2}$ & $\alpha k_{1}+\delta_{3} v_{1}$ & $\alpha k_{3}+\delta_{4} v_{3}$ & $\zeta k_{2}+\delta_{5} v_{2}$ & $\zeta k_{1}+\delta_{6} v_{1}$ \\
\hline$k_{1}$ & $k_{1}$ & 0 & $\delta_{7} v_{2}$ & $\delta_{8} v_{3}$ & $\delta_{9} v$ & $k_{2}+\delta_{10} v_{2}$ & $k_{3}+\delta_{11} v_{3}$ & $\delta_{12} v$ \\
\hline$k_{2}$ & $k_{2}$ & $k_{2}$ & $k_{1}+\delta_{13} v_{1}$ & $\delta_{14} v$ & $\delta_{15} v_{3}$ & $\delta_{16} v_{1}$ & $\delta_{17} v$ & $k_{3}+\delta_{1} 8 v_{3}$ \\
\hline$k_{3}$ & 0 & $k_{3}$ & $\delta_{19} v$ & $k_{1}+\delta_{20} v_{1}$ & $k_{2}+\delta_{21} v_{2}$ & $\delta_{22} v$ & $\delta_{23} v_{1}$ & $\delta_{24} v_{2}$ \\
\hline
\end{tabular}

Tabela 3.21: $O \mathfrak{s l}_{3}(k)-2$-módulo $U^{12}:=\left(V_{0} \curlywedge V_{3}^{*}\right) \curlywedge\left(V_{0} \curlywedge V_{3}\right)$.

Dessa tabela concluimos que

$$
U^{12} \simeq\left(V_{0} \wedge V_{3}^{*}\right) \oplus\left(V_{0} \curlywedge V_{3}\right) \Longleftrightarrow \delta_{i}=0, \forall i=1,2, \ldots, 24 .
$$

Além disso, por 3.2.2.4 e 3.2.2.2 temos que

$$
V_{0} \curlywedge V_{3}^{*} \simeq V_{0} \oplus V_{3}^{*} \Longleftrightarrow \delta=\alpha=\zeta=0
$$


e

$$
V_{0} \curlywedge V_{3} \simeq V_{0} \oplus V_{3} \Longleftrightarrow \omega=\theta=\sigma=0 .
$$

Portanto,

$$
U^{12} \simeq\left(V_{0} \oplus V_{3}^{*}\right) \oplus\left(V_{0} \oplus V_{3}\right) \Longleftrightarrow \delta_{i}=0=\omega=\theta=\sigma=\delta=\alpha=\zeta .
$$

nesse caso obtemos a seguinte tabela da $\mathfrak{s l}_{3}(k)$-ação sobre $U^{12}$ cindido.

\begin{tabular}{|c||c|c|c|c|c|c|c|c|}
\hline & $h_{1}$ & $h_{2}$ & $e_{12}$ & $e_{13}$ & $e_{23}$ & $e_{21}$ & $e_{31}$ & $e_{32}$ \\
\hline \hline$v$ & 0 & 0 & 0 & 0 & 0 & 0 & 0 & 0 \\
\hline$v_{1}$ & $v_{1}$ & 0 & $v_{2}$ & $v_{3}$ & 0 & 0 & 0 & 0 \\
\hline$v_{2}$ & $v_{2}$ & $v_{2}$ & 0 & 0 & $v_{3}$ & $v_{1}$ & 0 & 0 \\
\hline$v_{3}$ & 0 & $v_{3}$ & 0 & 0 & 0 & 0 & $v_{1}$ & $v_{2}$ \\
\hline$k$ & 0 & 0 & 0 & 0 & 0 & 0 & 0 & 0 \\
\hline$k_{1}$ & $k_{1}$ & 0 & 0 & 0 & 0 & $k_{2}$ & $k_{3}$ & 0 \\
\hline$k_{2}$ & $k_{2}$ & $k_{2}$ & $k_{1}$ & 0 & 0 & 0 & 0 & $k_{3}$ \\
\hline$k_{3}$ & 0 & $k_{3}$ & 0 & $k_{1}$ & $k_{2}$ & 0 & 0 & 0 \\
\hline
\end{tabular}

Tabela 3.22: $O \mathfrak{s l}_{3}(k)-2$-módulo $U^{12}:=\left(V_{0} \oplus V_{3}^{*}\right) \oplus\left(V_{0} \oplus V_{3}\right)$.

7. Tabela para o $\mathfrak{s l}_{3}(k)-2$-módulo $U^{13}:=\left(V_{3} \wedge V_{0}\right) \curlywedge\left(V_{0} \wedge V_{3}^{*}\right)$.

Seja $U^{13}:=\left\langle w, w_{1}, w_{2}, w_{3}, e, e_{1}, e_{2}, e_{3}\right\rangle \operatorname{logo}$ temos que a ação de $\mathfrak{s l}_{3}(k)$ sobre a base $\left\{w, w_{1}, w_{2}, w_{3}, e, e_{1}, e_{2}, e_{3}\right\}$ de $U^{13}$ é:

\begin{tabular}{|c||c|c|c|c|c|c|c|c|}
\hline & $h_{1}$ & $h_{2}$ & $e_{12}$ & $e_{13}$ & $e_{23}$ & $e_{21}$ & $e_{31}$ & $e_{32}$ \\
\hline \hline$w$ & 0 & 0 & $\delta w_{3}$ & $\delta w_{2}$ & $\alpha w_{1}$ & $\alpha w_{3}$ & $\zeta v_{2}$ & $\zeta v_{1}$ \\
\hline$w_{1}$ & $w_{1}$ & 0 & 0 & 0 & 0 & $w_{2}$ & $w_{3}$ & 0 \\
\hline$w_{2}$ & $w_{2}$ & $w_{2}$ & $w_{1}$ & 0 & 0 & 0 & 0 & $w_{3}$ \\
\hline$w_{3}$ & 0 & $w_{3}$ & 0 & $w_{1}$ & $w_{2}$ & 0 & 0 & 0 \\
\hline$e$ & 0 & 0 & $\delta_{1} w_{3}$ & $\delta_{1} w_{2}$ & $\delta_{2} w_{1}$ & $\delta_{2} w_{3}$ & $\delta_{3} w_{2}$ & $\delta_{3} w_{1}$ \\
\hline$e_{1}$ & $e_{1}$ & 0 & $e_{2}+\delta_{4} w_{2}$ & $e_{3}+\delta_{5} w_{3}$ & $\beta e+\delta_{6} w$ & $\delta_{7} w_{2}$ & $\delta_{8} w_{3}$ & $\lambda e+\delta_{9} w$ \\
\hline$e_{2}$ & $e_{2}$ & $e_{2}$ & $\delta_{4} w_{1}$ & $\gamma e+\delta_{12} w$ & $e_{3}+\delta_{10} w_{3}$ & $e_{1}+\delta_{7} w_{1}$ & $\lambda e+\delta_{9} w$ & $\delta_{11} w_{3}$ \\
\hline$e_{3}$ & 0 & $e_{3}$ & $\gamma e+\delta_{12} w$ & $\delta_{5} w_{1}$ & $\delta_{10} w_{2}$ & $\beta e+\delta_{6} w$ & $e_{1}+\delta_{8} w_{1}$ & $e_{2}+\delta_{11} w_{2}$ \\
\hline
\end{tabular}

Tabela 3.23: $O \mathfrak{s l}_{3}(k)-2-m o ́ d u l o U^{13}:=\left(V_{3}<V_{0}\right) \curlywedge\left(V_{0} \wedge V_{3}^{*}\right)$.

Dessa tabela observamos que: $U^{13} \simeq\left(V_{3} \wedge V_{0}\right) \oplus\left(V_{0} \wedge V_{3}^{*}\right) \Longleftrightarrow \delta_{i}=0, \forall i=1, \ldots, 12$ Agora, por 3.2.2.4 temos que

$$
V_{0} \curlywedge V_{3}^{*} \text { cinde } \Longleftrightarrow \delta=\alpha=\zeta=0 .
$$

Portanto:

$$
U^{13} \simeq\left(V_{3} \curlywedge V_{0}\right) \oplus\left(V_{0} \oplus V_{3}^{*}\right) \Longleftrightarrow \delta_{i}=0=\delta=\alpha=\zeta, \forall i=1, \ldots, 12 .
$$


8. Tabela para o $\mathfrak{s l}_{3}(k)-2-$ módulo $U^{15}:=\left(V_{0} \curlywedge V_{3}\right) \curlywedge\left(V_{0} \curlywedge V_{3}^{*}\right)$.

Seja $U^{15}:=\left\langle w, w_{1}, w_{2}, w_{3}, f, f_{1}, f_{2}, f_{3}\right\rangle$, logo temos que a ação de $\mathfrak{s l}_{3}(k)$ sobre a base $\left\{w, w_{1}, w_{2}, w_{3}, f, f_{1}, f_{2}, f_{3}\right\}$ de $U^{15}$ é:

\begin{tabular}{|c||c|c|c|c|c|c|c|c|}
\hline & $h_{1}$ & $h_{2}$ & $e_{12}$ & $e_{13}$ & $e_{23}$ & $e_{21}$ & $e_{31}$ & $e_{32}$ \\
\hline \hline$w$ & 0 & 0 & $\delta w_{3}$ & $\delta w_{2}$ & $\alpha w_{1}$ & $\alpha w_{3}$ & $\zeta v_{2}$ & $\zeta v_{1}$ \\
\hline$w_{1}$ & $w_{1}$ & 0 & 0 & 0 & 0 & $w_{2}$ & $w_{3}$ & 0 \\
\hline$w_{2}$ & $w_{2}$ & $w_{2}$ & $w_{1}$ & 0 & 0 & 0 & 0 & $w_{3}$ \\
\hline$w_{3}$ & 0 & $w_{3}$ & 0 & $w_{1}$ & $w_{2}$ & 0 & 0 & 0 \\
\hline$f$ & 0 & 0 & $\omega f_{3}+\delta_{1} w_{3}$ & $\theta f_{2}+\delta_{2} w_{2}$ & $\theta f_{1}+\delta_{3} w_{1}$ & $\sigma f_{3}+\delta_{4} w_{3}$ & $\sigma f_{2}+\delta_{5} w_{2}$ & $\alpha f_{1}+\delta_{6} w_{1}$ \\
\hline$f_{1}$ & $f_{1}$ & 0 & $f_{2}+\delta_{7} w_{2}$ & $f_{3}+\delta_{8} w_{3}$ & $\delta_{9} w$ & $\delta_{10} w_{2}$ & $\delta_{11} w_{3}$ & $\delta_{12} w$ \\
\hline$f_{2}$ & $f_{2}$ & $f_{2}$ & $\delta_{13} w_{1}$ & $\delta_{14} w$ & $f_{3}+\delta_{15} w_{3}$ & $f_{1}+\delta_{16} w_{1}$ & $\delta_{17} w$ & $\delta_{18} w_{3}$ \\
\hline$f_{3}$ & 0 & $f_{3}$ & $\delta_{19} w$ & $\delta_{20} w_{1}$ & $\delta_{21} w_{2}$ & $\delta_{22} w$ & $f_{1}+\delta_{23} w_{1}$ & $f_{2}+\delta_{24} w_{2}$ \\
\hline
\end{tabular}

Tabela 3.24: $O \mathfrak{s l}_{3}(k)-2$-módulo $U^{15}:=\left(V_{0} \curlywedge V_{3}\right) \curlywedge\left(V_{0} \curlywedge V_{3}^{*}\right)$.

Da tabela anterior tem-se:

$$
\left(V_{0} \wedge V_{3}\right) \wedge\left(V_{0} \wedge V_{3}^{*}\right) \simeq\left(V_{0} \wedge V_{3}\right) \oplus\left(V_{0} \wedge V_{3}^{*}\right)=0 \Longleftrightarrow \delta_{i}=0 \forall i=1, \ldots, 24 .
$$

Além disso, por 3.2.2.2 e 3.2.2.4 tem-se

$$
V_{0} \curlywedge V_{3} \simeq V_{0} \oplus V_{3} \Longleftrightarrow \omega=\theta=\sigma=0
$$

e

$$
V_{0} \curlywedge V_{3}^{*} \simeq V_{0} \oplus V_{3}^{*} \Longleftrightarrow \delta=\alpha=\zeta=0 .
$$

Portanto temos que:

$U^{15} \simeq\left(V_{0} \oplus V_{3}\right) \oplus\left(V_{0} \oplus V_{3}^{*}\right) \Longleftrightarrow \delta_{i}=0=\omega=\theta=\sigma=\delta=\alpha=\zeta, \forall i=1, \ldots, 24$. 
9. Tabela para o $\mathfrak{s l}_{3}(k)-2-$ módulo $U^{19}:=\left[V_{3}^{*} \curlywedge\left(V_{0} \curlywedge V_{0}\right)\right] \curlywedge V_{3}$

Seja $U^{19}:=\left\langle v_{1}, v_{2}, v_{3}, v, g, g_{1}, g_{2}, g_{3}\right\rangle$ logo por meio das tabelas 3.2 e 3.9 podemos obter que a ação $\mathfrak{s l}_{3}(k)$ sobre a base $\left\{v_{1}, v_{2}, v_{3}, v, g, g_{1}, g_{2}, g_{3}\right\}$ sobre $U^{19}$ é dada na tabela seguinte:

\begin{tabular}{|c||c|c|c|c|c|c|c|c|}
\hline & $h_{1}$ & $h_{2}$ & $e_{12}$ & $e_{13}$ & $e_{23}$ & $e_{21}$ & $e_{31}$ & $e_{32}$ \\
\hline \hline$v_{1}$ & $v_{1}$ & 0 & $v_{2}$ & $v_{3}$ & 0 & 0 & 0 & 0 \\
\hline$v_{2}$ & $v_{2}$ & $v_{2}$ & 0 & 0 & $v_{3}$ & $v_{1}$ & 0 & 0 \\
\hline$v_{3}$ & 0 & $v_{3}$ & 0 & 0 & 0 & 0 & $v_{1}$ & $v_{2}$ \\
\hline$v$ & 0 & 0 & $\delta_{1} v_{3}$ & $\delta_{2} v_{2}$ & $\delta_{3} v_{1}$ & $\delta_{4} v_{3}$ & $\delta_{5} v_{2}$ & $\delta_{6} v_{1}$ \\
\hline$g$ & 0 & 0 & $\delta_{7} v_{3}$ & $\delta_{8} v_{2}$ & $\delta_{9} v_{1}$ & $\delta_{10} v_{3}$ & $\delta_{11} v_{2}$ & $\delta_{12} v_{1}$ \\
\hline$g_{1}$ & $g_{1}$ & 0 & $\delta_{13} v_{2}$ & $\delta_{14} v_{3}$ & $\chi_{1} v+\chi_{2} g$ & $g_{2}+\delta_{15} v_{2}$ & $g_{3}+\delta_{16} v_{3}$ & $\chi_{3} v+\chi_{4} g$ \\
\hline$g_{2}$ & $g_{2}$ & $g_{2}$ & $g_{1}+\delta_{17} v_{1}$ & $\chi_{1} v+\chi_{2} g$ & $\delta_{18} v_{3}$ & $\delta_{19} v_{1}$ & $\chi_{5} v+\chi_{6} g$ & $g_{3}+\delta_{20} v_{3}$ \\
\hline$g_{3}$ & 0 & $g_{3}$ & $\chi_{3} v+\chi_{4} g$ & $g_{1}+\delta_{21} v_{1}$ & $g_{2}+\delta_{22} v_{2}$ & $\chi_{5} v+\chi_{6} g$ & $\delta_{23} v_{1}$ & $\delta_{24} v_{2}$ \\
\hline
\end{tabular}

Tabela 3.25: $O \mathfrak{s l}_{3}(k)-2$-módulo $U^{19}:=\left[V_{3}^{*} \curlywedge\left(V_{0} \curlywedge V_{0}\right)\right] \curlywedge V_{3}$

Da tabela anterior concluimos que

$$
U^{19} \simeq\left[V_{3}^{*} \curlywedge\left(V_{0} \curlywedge V_{0}\right)\right] \oplus V_{3} \Longleftrightarrow \delta_{i}=0 i=1, \ldots, 24 .
$$

nesse caso a ação de $\mathfrak{s l}_{3}(k)$ sobre $U^{19}$ cindido fica assim:

\begin{tabular}{|c||c|c|c|c|c|c|c|c|}
\hline & $h_{1}$ & $h_{2}$ & $e_{12}$ & $e_{13}$ & $e_{23}$ & $e_{21}$ & $e_{31}$ & $e_{32}$ \\
\hline \hline$v_{1}$ & $v_{1}$ & 0 & $v_{2}$ & $v_{3}$ & 0 & 0 & 0 & 0 \\
\hline$v_{2}$ & $v_{2}$ & $v_{2}$ & 0 & 0 & $v_{3}$ & $v_{1}$ & 0 & 0 \\
\hline$v_{3}$ & 0 & $v_{3}$ & 0 & 0 & 0 & 0 & $v_{1}$ & $v_{2}$ \\
\hline$v$ & 0 & 0 & $o$ & 0 & 0 & 0 & 0 & 0 \\
\hline$g$ & 0 & 0 & 0 & 0 & 0 & 0 & 0 & 0 \\
\hline$g_{1}$ & $g_{1}$ & 0 & 0 & 0 & $\chi_{1} v+\chi_{2} g$ & $g_{2}$ & $g_{3}$ & $\chi_{3} v+\chi_{4} g$ \\
\hline$g_{2}$ & $g_{2}$ & $g_{2}$ & $g_{1}$ & $\chi_{1} v+\chi_{2} g$ & 0 & 0 & $\chi_{5} v+\chi_{6} g$ & $g_{3}$ \\
\hline$g_{3}$ & 0 & $g_{3}$ & $\chi_{3} v+\chi_{4} g$ & $g_{1}$ & $g_{2}$ & $\chi_{5} v+\chi_{6} g$ & 0 & 0 \\
\hline
\end{tabular}

Tabela 3.26: $O \mathfrak{s l}_{3}(k)-2-m o ́ d u l o ~ U^{19}:=\left[V_{3}^{*} \wedge\left(V_{0} \wedge V_{0}\right)\right] \oplus V_{3}$

$\operatorname{com} \chi_{i} \in k$ para $i=1,2,3,4,5,6$. 
10. Tabela para o $\mathfrak{s l}_{3}(k)-2$-módulo $U^{20}:=\left[\left(V_{0} \curlywedge V_{0}\right) \curlywedge V_{3}^{*}\right] \curlywedge V_{3}$

Seja $U^{20}:=\left\langle v_{1}, v_{2}, v_{3}, i, j, i_{1}, i_{2}, i_{3}\right\rangle$. A tabela de ação de $\mathfrak{s l}_{3}(k)$ sobre a base $\left\{v_{1}, v_{2}, v_{3}, i, j, i_{1}, i_{2}, i_{3}\right\}$ de $U^{20}$ é dada por:

\begin{tabular}{|c||c|c|c|c|c|c|c|c|}
\hline & $h_{1}$ & $h_{2}$ & $e_{12}$ & $e_{13}$ & $e_{23}$ & $e_{21}$ & $e_{31}$ & $e_{32}$ \\
\hline \hline$v_{1}$ & $v_{1}$ & 0 & $v_{2}$ & $v_{3}$ & 0 & 0 & 0 & 0 \\
\hline$v_{2}$ & $v_{2}$ & $v_{2}$ & 0 & 0 & $v_{3}$ & $v_{1}$ & 0 & 0 \\
\hline$v_{3}$ & 0 & $v_{3}$ & 0 & 0 & 0 & 0 & $v_{1}$ & $v_{2}$ \\
\hline$i$ & 0 & 0 & $\tau i_{3}+\delta_{1} v_{3}$ & $\tau i_{2}+\delta_{2} v_{2}$ & $\rho i_{1}+\delta_{3} v_{1}$ & $\rho i_{3}+\delta_{4} v_{3}$ & $\varrho i_{2}+\delta_{5} v_{2}$ & $\varrho i_{1}+\delta_{6} v_{1}$ \\
\hline$j$ & 0 & 0 & $\tau_{1} j_{3}+\delta_{7} v_{3}$ & $\tau_{1} j_{2}+\delta_{8} v_{2}$ & $\rho_{1} j_{1}+\delta_{9} v_{1}$ & $\rho_{1}+j_{3} \delta_{10} v_{3}$ & $\varrho_{1} j_{2}+\delta_{11} v_{2}$ & $\varrho_{1} j_{1}+\delta_{12} v_{1}$ \\
\hline$i_{1}$ & $i_{1}$ & 0 & $\delta_{13} v_{2}$ & $\delta_{14} v_{3}$ & 0 & $i_{2}+\delta_{15} v_{2}$ & $i_{3}+\delta_{16} v_{3}$ & 0 \\
\hline$i_{2}$ & $i_{2}$ & $i_{2}$ & $i_{1}+\delta_{17} v_{1}$ & 0 & $\delta_{18} v_{3}$ & $\delta_{19} v_{1}$ & 0 & $i_{3}+\delta_{20} v_{3}$ \\
\hline$i_{3}$ & 0 & $i_{3}$ & 0 & $i_{1}+\delta_{21} v_{1}$ & $i_{2}+\delta_{22} v_{2}$ & 0 & $\delta_{23} v_{1}$ & $\delta_{24} v_{2}$ \\
\hline
\end{tabular}

Tabela 3.27: $O \mathfrak{s l}_{3}(k)$-2-módulo $U^{20}:=\left[\left(V_{0} \wedge V_{0}\right) \curlywedge V_{3}^{*}\right] \curlywedge V_{3}$

Da tabela anterior

$U^{20} \simeq\left[\left(V_{0} \wedge V_{0}\right) \oplus V_{3}^{*}\right] \oplus V_{3} \Longleftrightarrow \delta_{i}=\tau=\rho=\varrho=\tau_{1}=\rho_{1}=\varrho_{1}=0, \quad i=1,2, \ldots, 24$

11. Tabela para o $\mathfrak{s l}_{3}(k)-2$-módulo $U^{25}:=\left[\left(V_{3}^{*} \curlywedge V_{0}\right) \curlywedge V_{0}\right] \curlywedge V_{3}$

Seja $U^{25}:=\left\langle i, v_{1}, v_{2}, v_{3}, j, j_{1}, j_{2}, j_{3}\right\rangle$ a tabela de ação de $\mathfrak{s l}_{3}(k)$ sobre $U^{25}$ é dada assim:

\begin{tabular}{|c||c|c|c|c|c|c|c|c|}
\hline & $h_{1}$ & $h_{2}$ & $e_{12}$ & $e_{13}$ & $e_{23}$ & $e_{21}$ & $e_{31}$ & $e_{32}$ \\
\hline \hline$v_{1}$ & $v_{1}$ & 0 & $v_{2}$ & $v_{3}$ & 0 & 0 & 0 & 0 \\
\hline$v_{2}$ & $v_{2}$ & $v_{2}$ & 0 & 0 & $v_{3}$ & $v_{1}$ & 0 & 0 \\
\hline$v_{3}$ & 0 & $v_{3}$ & 0 & 0 & 0 & 0 & $v_{1}$ & $v_{2}$ \\
\hline$i$ & 0 & 0 & $\delta_{1} v_{3}$ & $\delta_{2} v_{2}$ & $\delta_{3} v_{1}$ & $\delta_{4} v_{3}$ & $\delta_{5} v_{2}$ & $\delta_{6} v_{1}$ \\
\hline$j$ & 0 & 0 & $\delta_{7} v_{3}$ & $\delta_{8} v_{2}$ & $\delta_{9} v_{1}$ & $\delta_{10} v_{3}$ & $\delta_{11} v_{2}$ & $\delta_{12} v_{1}$ \\
\hline$j_{1}$ & $j_{1}$ & 0 & $\delta_{13} v_{2}$ & $\delta_{14} v_{3}$ & $\pi j+\triangle_{1} i$ & $j_{2}+\delta_{15} v_{2}$ & $j_{3}+\delta_{16} v_{3}$ & $\lambda j+\triangle_{2} i$ \\
\hline$j_{2}$ & $j_{2}$ & $j_{2}$ & $j_{1}+\delta_{17} v_{1}$ & $\pi j+\triangle_{3} i$ & $\delta_{18} v_{3}$ & $\delta_{19} v_{1}$ & $\xi j+\triangle_{4} i$ & $j_{3}+\delta_{20} v_{3}$ \\
\hline$j_{3}$ & 0 & $j_{3}$ & $\lambda j+\triangle_{5} i$ & $j_{1}+\delta_{21} v_{1}$ & $j_{2}+\delta_{22} v_{2}$ & $\xi j+\triangle_{6} v$ & $\delta_{23} v_{1}$ & $\delta_{24} v_{2}$ \\
\hline
\end{tabular}

Tabela 3.28: $O \mathfrak{s l}_{3}(k)-2$-módulo $U^{25}:=\left[\left(V_{3}^{*} \curlywedge V_{0}\right) \curlywedge V_{0}\right] \curlywedge V_{3}$

Da tabela anterior

$$
U^{25} \simeq\left[\left(V_{3}^{*} \curlywedge V_{0}\right) \oplus V_{0}\right] \oplus V_{3} \Longleftrightarrow \delta_{i}=\triangle_{j}=0, \quad i=1,2, \ldots, 24 \text { e } j=1, \ldots, 6 .
$$


12. Tabela para o $\mathfrak{s l}_{3}(k)$-2-módulo $U^{26}:=\left[V_{0} \curlywedge\left(V_{3}^{*} \curlywedge V_{0}\right)\right] \curlywedge V_{3}$

Seja $U^{26}:=\left\langle v_{1}, v_{2}, v_{3}, k, k_{1}, k_{2}, k_{3}, x\right\rangle$ onde a ação de $\mathfrak{s l}_{3}(k)$ sobre a base $\left\{k, x, v_{1}, v_{2}, v_{3}, k_{1}, k_{2}, k_{3}\right\}$ de $U^{26}$ é dada por:

\begin{tabular}{|c||c|c|c|c|c|c|c|c|}
\hline & $h_{1}$ & $h_{2}$ & $e_{12}$ & $e_{13}$ & $e_{23}$ & $e_{21}$ & $e_{31}$ & $e_{32}$ \\
\hline \hline$v_{1}$ & $v_{1}$ & 0 & $v_{2}$ & $v_{3}$ & 0 & 0 & 0 & 0 \\
\hline$v_{2}$ & $v_{2}$ & $v_{2}$ & 0 & 0 & $v_{3}$ & $v_{1}$ & 0 & 0 \\
\hline$v_{3}$ & 0 & $v_{3}$ & 0 & 0 & 0 & 0 & $v_{1}$ & $v_{2}$ \\
\hline$k$ & 0 & 0 & $\delta_{1} v_{3}$ & $\delta_{2} v_{2}$ & $\delta_{3} v_{1}$ & $\delta_{4} v_{3}$ & $\delta_{5} v_{2}$ & $\delta_{6} v_{1}$ \\
\hline$k_{1}$ & $k_{1}$ & 0 & $\delta_{7} v_{2}$ & $\delta_{8} v_{3}$ & $\pi k$ & $k_{2}+\delta_{9} v_{2}$ & $k_{3}+\delta_{10} v_{3}$ & $\lambda k$ \\
\hline$k_{2}$ & $k_{2}$ & $k_{2}$ & $k_{1}+\delta_{11} v_{1}$ & $\pi k$ & $\delta_{12} v_{3}$ & $\delta_{13} v_{1}$ & $\xi k$ & $k_{3}+\delta_{14} v_{3}$ \\
\hline$k_{3}$ & 0 & $k_{3}$ & $\lambda k$ & $k_{1}+\delta_{15} v_{1}$ & $k_{2}+\delta_{16} v_{2}$ & $\xi k$ & $\delta_{17} v_{1}$ & $\delta_{18} v_{2}$ \\
\hline$x$ & 0 & 0 & $\psi k_{3}+\delta_{19} v_{3}$ & $\psi k_{2}+\delta_{20} v_{2}$ & $\varphi k_{1}+\delta_{21} v_{1}$ & $\varphi k_{3}+\delta_{22} v_{3}$ & $o k_{2}+\delta_{23} v_{2}$ & $o k_{1}+\delta_{24} v_{1}$ \\
\hline
\end{tabular}

Tabela 3.29: $O \mathfrak{s l}_{3}(k)-2$-módulo $U^{26}:=\left[V_{0} \curlywedge\left(V_{3}^{*} \curlywedge V_{0}\right)\right] \curlywedge V_{3}$

Da tabela anterior obtemos que:

$$
U^{26} \simeq\left[V_{0} \oplus\left(V_{3}^{*} \curlywedge V_{0}\right)\right] \oplus V_{3} \Longleftrightarrow \delta_{i}=0=\psi=\varphi=o
$$

13. Tabela para o $\mathfrak{s l}_{3}(k)-2-$ módulo $U^{27}:=\left[\left(V_{0} \curlywedge V_{3}^{*}\right) \curlywedge V_{0}\right] \curlywedge V_{3}$

\begin{tabular}{|c||c|c|c|c|c|c|c|c|}
\hline & $h_{1}$ & $h_{2}$ & $e_{12}$ & $e_{13}$ & $e_{23}$ & $e_{21}$ & $e_{31}$ & $e_{32}$ \\
\hline \hline$v_{1}$ & $v_{1}$ & 0 & $v_{2}$ & $v_{3}$ & 0 & 0 & 0 & 0 \\
\hline$v_{2}$ & $v_{2}$ & $v_{2}$ & 0 & 0 & $v_{3}$ & $v_{1}$ & 0 & 0 \\
\hline$v_{3}$ & 0 & $v_{3}$ & 0 & 0 & 0 & 0 & $v_{1}$ & $v_{2}$ \\
\hline$y$ & 0 & 0 & $\delta y_{3}+\delta_{1} v_{3}$ & $\delta y_{2}+\delta_{2} v_{2}$ & $\alpha y_{1}+\delta_{3} v_{1}$ & $\alpha y_{3}+\delta_{4} v_{3}$ & $\zeta y_{2}+\delta_{5} v_{2}$ & $\zeta y_{1}+\delta_{6} v_{1}$ \\
\hline$y_{1}$ & $y_{1}$ & 0 & $\delta_{7} v_{2}$ & $\delta_{8} v_{3}$ & $\lambda_{1} z$ & $y_{2}+\delta_{9} v_{2}$ & $y_{3}+\delta_{10} v_{3}$ & $\lambda_{2} z$ \\
\hline$y_{2}$ & $y_{2}$ & $y_{2}$ & $y_{1}+\delta_{11} v_{1}$ & $\lambda_{3} z$ & $\delta_{12} v_{3}$ & $\delta_{13} v_{1}$ & $\lambda_{4} z$ & $y_{3}+\delta_{14} v_{3}$ \\
\hline$y_{3}$ & 0 & $y_{3}$ & $\lambda_{5} z$ & $y_{1}+\delta_{15} v_{1}$ & $y_{2}+\delta_{16} v_{2}$ & $\lambda_{6} z$ & $\delta_{17} v_{1}$ & $\delta_{18} v_{2}$ \\
\hline$z$ & 0 & 0 & 0 & 0 & 0 & 0 & 0 & 0 \\
\hline
\end{tabular}

Tabela 3.30: $O \mathfrak{s l}_{3}(k)$-2-módulo $U^{27}:=\left[\left(V_{0} \curlywedge V_{3}^{*}\right) \curlywedge V_{0}\right] \curlywedge V_{3}$

Dessa tabela tem-se:

$$
U^{27} \simeq\left[\left(V_{0} \oplus V_{3}^{*}\right) \oplus V_{0}\right] \oplus V_{3} \Longleftrightarrow \delta_{i}=0=\lambda_{j}=\delta=\alpha=\zeta
$$


14. Tabela para o $\mathfrak{s l}_{3}(k)-2$-módulo $U^{28}:=\left[V_{0} \curlywedge\left(V_{0} \wedge V_{3}^{*}\right)\right] \curlywedge V_{3}$

\begin{tabular}{|c||c|c|c|c|c|c|c|c|}
\hline & $h_{1}$ & $h_{2}$ & $e_{12}$ & $e_{13}$ & $e_{23}$ & $e_{21}$ & $e_{31}$ & $e_{32}$ \\
\hline \hline$v_{1}$ & $v_{1}$ & 0 & $v_{2}$ & $v_{3}$ & 0 & 0 & 0 & 0 \\
\hline$v_{2}$ & $v_{2}$ & $v_{2}$ & 0 & 0 & $v_{3}$ & $v_{1}$ & 0 & 0 \\
\hline$v_{3}$ & 0 & $v_{3}$ & 0 & 0 & 0 & 0 & $v_{1}$ & $v_{2}$ \\
\hline$b$ & 0 & 0 & $\delta b_{3}+\delta_{1} v_{3}$ & $\delta b_{2}+\delta_{2} v_{2}$ & $\alpha b_{1}+\delta_{3} v_{1}$ & $\alpha b_{3}+\delta_{4} v_{3}$ & $\zeta b_{2}+\delta_{5} v_{2}$ & $\zeta b_{1}+\delta_{6} v_{1}$ \\
\hline$b_{1}$ & $b_{1}$ & 0 & $\delta_{7} v_{2}$ & $\delta_{8} v_{3}$ & 0 & $b_{2}+\delta_{9} v_{2}$ & $b_{3}+\delta_{10} v_{3}$ & 0 \\
\hline$b_{2}$ & $b_{2}$ & $b_{2}$ & $b_{1}+\delta_{11} v_{1}$ & 0 & $\delta_{12} v_{3}$ & $\delta_{13} v_{1}$ & 0 & $b_{3}+\delta_{14} v_{3}$ \\
\hline$b_{3}$ & 0 & $b_{3}$ & 0 & $b_{1}+\delta_{15} v_{1}$ & $b_{2}+\delta_{16} v_{2}$ & 0 & $\delta_{17} v_{1}$ & $\delta_{18} v_{2}$ \\
\hline$f$ & 0 & 0 & $\phi_{2} b_{3}+\delta_{19} v_{3}$ & $\phi_{2} b_{2}+\delta_{20} v_{2}$ & $\phi_{2} b_{1}+\delta_{21} v_{1}$ & $\phi_{2} b_{3}+\delta_{22} v_{3}$ & $\phi_{3} b_{2}+\delta_{23} v_{2}$ & $\phi_{3} b_{1}+\delta_{24} v_{1}$ \\
\hline
\end{tabular}

Tabela 3.31: $O \mathfrak{s l}_{3}(k)$-2-módulo $U^{28}:=\left[V_{0} \wedge\left(V_{0} \wedge V_{3}^{*}\right)\right] \curlywedge V_{3}$

Desta tabela:

$$
U^{28} \simeq\left[V_{0} \oplus\left(V_{0} \oplus V_{3}^{*}\right)\right] \oplus V_{3} \Longleftrightarrow \delta_{i}=\delta=\alpha=\zeta=\phi_{i}=0
$$

15. Tabela para o $\mathfrak{s l}_{3}(k)-2-$ módulo $U^{31}:=V_{3} \wedge\left[V_{3}^{*} \wedge\left(V_{0} \curlywedge V_{0}\right)\right]$

Seja $\left\{v, w, w_{1}, w_{2}, w_{3}, v_{1}, v_{2}, v_{3}\right\}$ base de $U^{31}$, logo a ação de $\mathfrak{s l}_{3}(k)$ sobre $U^{31}$ é:

\begin{tabular}{|c||c|c|c|c|c|c|c|c|}
\hline & $h_{1}$ & $h_{2}$ & $e_{12}$ & $e_{13}$ & $e_{23}$ & $e_{21}$ & $e_{31}$ & $e_{32}$ \\
\hline \hline$v$ & 0 & 0 & 0 & 0 & 0 & 0 & 0 & 0 \\
\hline$w$ & 0 & 0 & 0 & 0 & 0 & 0 & 0 & 0 \\
\hline$w_{1}$ & $w_{1}$ & 0 & 0 & 0 & $\chi_{1} v+\chi_{2} w$ & $w_{2}$ & $w_{3}$ & $\chi_{3} v+\chi_{4} w$ \\
\hline$w_{2}$ & $w_{2}$ & $w_{2}$ & $w_{1}$ & $\chi_{1} v+\chi_{2} w$ & 0 & 0 & $\chi_{5} v+\chi_{6} w$ & $w_{3}$ \\
\hline$w_{3}$ & 0 & $w_{3}$ & $\chi_{3} v+\chi_{4} w$ & $w_{1}$ & $w_{2}$ & $\chi_{5} v+\chi_{6} w$ & 0 & 0 \\
\hline$v_{1}$ & $v_{1}$ & 0 & $v_{2}+\delta_{1} w_{2}$ & $v_{3}+\delta_{2} w_{3}$ & $\delta_{3} v+\delta_{4} w$ & $\delta_{5} w_{2}$ & $\delta_{6} w_{3}$ & $\delta_{7} v+\delta_{8} w$ \\
\hline$v_{2}$ & $v_{2}$ & $v_{2}$ & $\delta_{9} w_{1}$ & $\delta_{10} v+\delta_{11} w$ & $v_{3}+\delta_{12} w_{3}$ & $v_{1}+\delta_{13} w_{1}$ & $\delta_{14} v+\delta_{15} w$ & $\delta_{16} w_{3}$ \\
\hline$v_{3}$ & 0 & $v_{3}$ & $\delta_{17} v+\delta_{18} w$ & $\delta_{19} w_{1}$ & $\delta_{20} w_{2}$ & $\delta_{21} v+\delta_{22} w$ & $v_{1}+\delta_{23} w_{1}$ & $v_{2}+\delta_{29} w_{2}$ \\
\hline
\end{tabular}

Tabela 3.32: $O \mathfrak{s l}_{3}(k)-2-m o ́ d u l o ~ U^{31}:=V_{3} \wedge\left[V_{3}^{*} \wedge\left(V_{0} \wedge V_{0}\right)\right]$

Da tabela anterior temos que:

$$
U^{31} \simeq V_{3} \oplus\left[V_{3}^{*} \curlywedge\left(V_{0} \curlywedge V_{0}\right)\right] \Longleftrightarrow \delta_{i}=0 .
$$


16. Tabela para o $\mathfrak{s l}_{3}(k)-2$-módulo $U^{32}:=V_{3} \wedge\left[\left(V_{0} \wedge V_{0}\right) \wedge V_{3}^{*}\right]$

\begin{tabular}{|c||c|c|c|c|c|c|c|c|}
\hline & $h_{1}$ & $h_{2}$ & $e_{12}$ & $e_{13}$ & $e_{23}$ & $e_{21}$ & $e_{31}$ & $e_{32}$ \\
\hline \hline$w_{1}$ & $w_{1}$ & 0 & 0 & 0 & 0 & $w_{2}$ & $w_{3}$ & 0 \\
\hline$w_{2}$ & $w_{2}$ & $w_{2}$ & $w_{1}$ & 0 & 0 & 0 & 0 & $w_{3}$ \\
\hline$w_{3}$ & 0 & $w_{3}$ & 0 & $w_{1}$ & $w_{2}$ & 0 & 0 & 0 \\
\hline$w$ & 0 & 0 & $\tau w_{3}$ & $\tau w_{2}$ & $\rho w_{1}$ & $\rho_{3}$ & $\varrho_{3}$ & $\varrho w_{1}$ \\
\hline$v$ & 0 & 0 & $\tau_{1} w_{3}$ & $\tau_{1} w_{2}$ & $\rho_{1} w_{1}$ & $\rho_{1} w_{3}$ & $\varrho_{1} w_{2}$ & $\varrho_{1} w_{1}$ \\
\hline$v_{1}$ & $v_{1}$ & 0 & $v_{2}+\delta_{1} w_{2}$ & $v_{3}+\delta_{2} w_{3}$ & $\delta_{3} v+\delta_{4} w$ & $\delta_{5} w_{2}$ & $\delta_{6} w_{3}$ & $\delta_{7} v+\delta_{8} w$ \\
\hline$v_{2}$ & $v_{2}$ & $v_{2}$ & $\delta_{9} w_{1}$ & $\delta_{10} v+\delta_{11} w$ & $v_{3}+\delta_{12} w_{3}$ & $v_{1}+\delta_{13} w_{1}$ & $\delta_{14} v+\delta_{15} w$ & $\delta_{16} w_{3}$ \\
\hline$v_{3}$ & 0 & $v_{3}$ & $\delta_{17} v+\delta_{18} w$ & $\delta_{19} w_{1}$ & $\delta_{20} w_{2}$ & $\delta_{21} v+\delta_{22} w$ & $v_{1}+\delta_{23} w_{1}$ & $v_{2}+\delta_{29} w_{2}$ \\
\hline
\end{tabular}

Tabela 3.33: $O \mathfrak{s l}_{3}(k)$-2-módulo $U^{32}:=V_{3} \wedge\left[\left(V_{0} \wedge V_{0}\right) \wedge V_{3}^{*}\right]$

Da tabela anterior temos que:

$$
U^{32} \simeq V_{3} \oplus\left[\left(V_{0} \wedge V_{0}\right) \oplus V_{3}^{*}\right] \Longleftrightarrow \delta_{i}=\tau=\rho=\varrho=\tau_{1}=\rho_{1}=\varrho_{1}=0 .
$$

17. Tabela para o $\mathfrak{s l}_{3}(k)-2-$ módulo $U^{37}:=V_{3} \curlywedge\left[\left(V_{3}^{*} \wedge V_{0}\right) \curlywedge V_{0}\right]$

\begin{tabular}{|c||c|c|c|c|c|c|c|c|}
\hline & $h_{1}$ & $h_{2}$ & $e_{12}$ & $e_{13}$ & $e_{23}$ & $e_{21}$ & $e_{31}$ & $e_{32}$ \\
\hline \hline$v$ & 0 & 0 & 0 & 0 & 0 & 0 & 0 & 0 \\
\hline$w$ & 0 & 0 & 0 & 0 & 0 & 0 & 0 & 0 \\
\hline$w_{1}$ & $w_{1}$ & 0 & 0 & 0 & $\pi w+\triangle_{1} v$ & $w_{2}$ & $w_{3}$ & $\lambda w+\triangle_{2} v$ \\
\hline$w_{2}$ & $w_{2}$ & $w_{2}$ & $w_{1}$ & $\pi w+\triangle_{3} v$ & 0 & 0 & $\xi w+\triangle_{4} v$ & $w_{3}$ \\
\hline$w_{3}$ & 0 & $w_{3}$ & $\lambda w+\triangle_{5} v$ & $w_{1}$ & $w_{2}$ & $\xi w+\triangle_{6} v$ & 0 & 0 \\
\hline$v_{1}$ & $v_{1}$ & 0 & $v_{2}+\delta_{1} w_{2}$ & $v_{3}+\delta_{2} w_{3}$ & $\delta_{3} v+\delta_{4} w$ & $\delta_{5} w_{2}$ & $\delta_{6} w_{3}$ & $\delta_{7} v+\delta_{8} w$ \\
\hline$v_{2}$ & $v_{2}$ & $v_{2}$ & $\delta_{9} w_{1}$ & $\delta_{10} v+\delta_{11} w$ & $v_{3}+\delta_{12} w_{3}$ & $v_{1}+\delta_{13} w_{1}$ & $\delta_{14} v+\delta_{15} w$ & $\delta_{16} w_{3}$ \\
\hline$v_{3}$ & 0 & $v_{3}$ & $\delta_{17} v+\delta_{18} w$ & $\delta_{19} w_{1}$ & $\delta_{20} w_{2}$ & $\delta_{21} v+\delta_{22} w$ & $v_{1}+\delta_{23} w_{1}$ & $v_{2}+\delta_{29} w_{2}$ \\
\hline
\end{tabular}

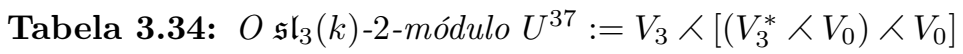

Da tabela anterior temos que:

$$
U^{37} \simeq V_{3} \oplus\left[\left(V_{3}^{*} \wedge V_{0}\right) \oplus V_{0}\right] \Longleftrightarrow \delta_{i}=\Delta_{j}=0 .
$$


18. Tabela para o $\mathfrak{s l}_{3}(k)-2$-módulo $U^{38}:=V_{3} \curlywedge\left[V_{0} \curlywedge\left(V_{3}^{*} \curlywedge V_{0}\right)\right]$

\begin{tabular}{|c||c|c|c|c|c|c|c|c|}
\hline & $h_{1}$ & $h_{2}$ & $e_{12}$ & $e_{13}$ & $e_{23}$ & $e_{21}$ & $e_{31}$ & $e_{32}$ \\
\hline \hline$w$ & 0 & 0 & 0 & 0 & 0 & 0 & 0 & 0 \\
\hline$w_{1}$ & $w_{1}$ & 0 & 0 & 0 & $\pi w$ & $w_{2}$ & $w_{3}$ & $\lambda w$ \\
\hline$w_{2}$ & $w_{2}$ & $w_{2}$ & $w_{1}$ & $\pi w$ & 0 & 0 & $\xi w$ & $u_{3}$ \\
\hline$w_{3}$ & 0 & $w_{3}$ & $\lambda w$ & $w_{1}$ & $w_{2}$ & $\xi w$ & 0 & 0 \\
\hline$v$ & 0 & 0 & $\psi w_{3}$ & $\psi w_{2}$ & $\varphi w_{1}$ & $\varphi w_{3}$ & $o w_{2}$ & $o w_{1}$ \\
\hline$v_{1}$ & $v_{1}$ & 0 & $v_{2}+\delta_{1} w_{2}$ & $v_{3}+\delta_{2} w_{3}$ & $\delta_{3} v+\delta_{4} w$ & $\delta_{5} w_{2}$ & $\delta_{6} w_{3}$ & $\delta_{7} v+\delta_{8} w$ \\
\hline$v_{2}$ & $v_{2}$ & $v_{2}$ & $\delta_{9} w_{1}$ & $\delta_{10} v+\delta_{11} w$ & $v_{3}+\delta_{12} w_{3}$ & $v_{1}+\delta_{13} w_{1}$ & $\delta_{14} v+\delta_{15} w$ & $\delta_{16} w_{3}$ \\
\hline$v_{3}$ & 0 & $v_{3}$ & $\delta_{17} v+\delta_{18} w$ & $\delta_{19} w_{1}$ & $\delta_{20} w_{2}$ & $\delta_{21} v+\delta_{22} w$ & $v_{1}+\delta_{23} w_{1}$ & $v_{2}+\delta_{29} w_{2}$ \\
\hline
\end{tabular}

Tabela 3.35: $O \mathfrak{s l}_{3}(k)-2$-módulo $U^{38}:=V_{3} \wedge\left[V_{0} \wedge\left(V_{3}^{*} \curlywedge V_{0}\right)\right]$

Da tabela anterior temos que:

$$
U^{38} \simeq V_{3} \oplus\left[V_{0} \oplus\left(V_{3}^{*} \curlywedge V_{0}\right)\right] \Longleftrightarrow \delta_{i}=\phi=o=\varphi=0 .
$$

19. Tabela para o $\mathfrak{s l}_{3}(k)-2-$ módulo $U^{39}:=V_{3} \wedge\left[\left(V_{0} \wedge V_{3}^{*}\right) \curlywedge V_{0}\right]$

\begin{tabular}{|c||c|c|c|c|c|c|c|c|}
\hline & $h_{1}$ & $h_{2}$ & $e_{12}$ & $e_{13}$ & $e_{23}$ & $e_{21}$ & $e_{31}$ & $e_{32}$ \\
\hline \hline$w$ & 0 & 0 & $\delta w_{3}$ & $\delta w_{2}$ & $\alpha w_{1}$ & $\alpha w_{3}$ & $\zeta w_{2}$ & $\zeta w_{1}$ \\
\hline$w_{1}$ & $w_{1}$ & 0 & 0 & 0 & $\lambda_{1} v$ & $w_{2}$ & $w_{3}$ & $\lambda_{2} v$ \\
\hline$w_{2}$ & $w_{2}$ & $w_{2}$ & $w_{1}$ & $\lambda_{3} v$ & 0 & 0 & $\lambda_{4} v$ & $w_{3}$ \\
\hline$w_{3}$ & 0 & $w_{3}$ & $\lambda_{5} v$ & $w_{1}$ & $w_{2}$ & $\lambda_{6} v$ & 0 & 0 \\
\hline$v$ & 0 & 0 & 0 & 0 & 0 & 0 & 0 & 0 \\
\hline$v_{1}$ & $v_{1}$ & 0 & $v_{2}+\delta_{1} w_{2}$ & $v_{3}+\delta_{2} w_{3}$ & $\delta_{3} v+\delta_{4} w$ & $\delta_{5} w_{2}$ & $\delta_{6} w_{3}$ & $\delta_{7} v+\delta_{8} w$ \\
\hline$v_{2}$ & $v_{2}$ & $v_{2}$ & $\delta_{9} w_{1}$ & $\delta_{10} v+\delta_{11} w$ & $v_{3}+\delta_{12} w_{3}$ & $v_{1}+\delta_{13} w_{1}$ & $\delta_{14} v+\delta_{15} w$ & $\delta_{16} w_{3}$ \\
\hline$v_{3}$ & 0 & $v_{3}$ & $\delta_{17} v+\delta_{18} w$ & $\delta_{19} w_{1}$ & $\delta_{20} w_{2}$ & $\delta_{21} v+\delta_{22} w$ & $v_{1}+\delta_{23} w_{1}$ & $v_{2}+\delta_{29} w_{2}$ \\
\hline
\end{tabular}

Tabela 3.36: $O \mathfrak{s l}_{3}(k)-2$-módulo $U^{39}:=V_{3} \curlywedge\left[\left(V_{0} \curlywedge V_{3}^{*}\right) \curlywedge V_{0}\right]$

Da tabela anterior temos que:

$$
U^{39} \simeq V_{3} \oplus\left[\left(V_{0} \oplus V_{3}^{*}\right) \oplus V_{0}\right] \Longleftrightarrow \delta_{i}=\alpha=\zeta=\delta=\lambda_{j}=0 .
$$


20. Tabela para o $\mathfrak{s l}_{3}(k)-2-$ módulo $U^{40}:=V_{3} \curlywedge\left[V_{0} \curlywedge\left(V_{0} \curlywedge V_{3}^{*}\right)\right]$

\begin{tabular}{|c||c|c|c|c|c|c|c|c|}
\hline & $h_{1}$ & $h_{2}$ & $e_{12}$ & $e_{13}$ & $e_{23}$ & $e_{21}$ & $e_{31}$ & $e_{32}$ \\
\hline \hline$w$ & 0 & 0 & $\delta w_{3}$ & $\delta w_{2}$ & $\alpha w_{1}$ & $\alpha w_{3}$ & $\zeta w_{2}$ & $\zeta w_{1}$ \\
\hline$w_{1}$ & $w_{1}$ & 0 & 0 & 0 & 0 & $w_{2}$ & $w_{3}$ & 0 \\
\hline$w_{2}$ & $w_{2}$ & $w_{2}$ & $w_{1}$ & 0 & 0 & 0 & 0 & $w_{3}$ \\
\hline$w_{3}$ & 0 & $w_{3}$ & 0 & $w_{1}$ & $w_{2}$ & 0 & 0 & 0 \\
\hline$v$ & 0 & 0 & $\phi_{1} w_{3}$ & $\phi_{1} w_{2}$ & $\phi_{2} w_{1}$ & $\phi_{2} w_{3}$ & $\phi_{3} w_{2}$ & $\phi_{3} w_{1}$ \\
\hline$v_{1}$ & $v_{1}$ & 0 & $v_{2}+\delta_{1} w_{2}$ & $v_{3}+\delta_{2} w_{3}$ & $\delta_{3} v+\delta_{4} w$ & $\delta_{5} w_{2}$ & $\delta_{6} w_{3}$ & $\delta_{7} v+\delta_{8} w$ \\
\hline$v_{2}$ & $v_{2}$ & $v_{2}$ & $\delta_{9} w_{1}$ & $\delta_{10} v+\delta_{11} w$ & $v_{3}+\delta_{12} w_{3}$ & $v_{1}+\delta_{13} w_{1}$ & $\delta_{14} v+\delta_{15} w$ & $\delta_{16} w_{3}$ \\
\hline$v_{3}$ & 0 & $v_{3}$ & $\delta_{17} v+\delta_{18} w$ & $\delta_{19} w_{1}$ & $\delta_{20} w_{2}$ & $\delta_{21} v+\delta_{22} w$ & $v_{1}+\delta_{23} w_{1}$ & $v_{2}+\delta_{29} w_{2}$ \\
\hline
\end{tabular}

Tabela 3.37: $O \mathfrak{s l}_{3}(k)$-2-módulo $U^{40}:=V_{3} \wedge\left[V_{0} \wedge\left(V_{0}<V_{3}^{*}\right)\right]$

Da tabela anterior temos que:

$$
U^{40} \simeq V_{3} \curlywedge\left[V_{0} \curlywedge\left(V_{0} \curlywedge V_{3}^{*}\right)\right] \Longleftrightarrow \delta_{i}=\alpha=\zeta=\delta=\phi_{j}=0 .
$$

21. Tabela para o $\mathfrak{s l}_{3}(k)-2-$ módulo $U^{41}:=\left[V_{3} \wedge\left(V_{0} \wedge V_{0}\right)\right] \wedge V_{3}^{*}$

\begin{tabular}{|c|c|c|c|c|c|c|c|c|}
\hline & $h_{1}$ & $h_{2}$ & $e_{12}$ & $e_{13}$ & $e_{23}$ & $e_{21}$ & $e_{31}$ & $e_{32}$ \\
\hline$v$ & 0 & 0 & $\delta_{1} w_{3}$ & $\delta_{2} w_{2}$ & $\delta_{3} w_{1}$ & $\delta_{4} w_{3}$ & $\delta_{5} w_{2}$ & $\delta_{6} w_{1}$ \\
\hline$w$ & 0 & 0 & $\delta_{7} w_{3}$ & $\delta_{8} w_{2}$ & $\delta_{9} w_{1}$ & $\delta_{10} w_{3}$ & $\delta_{11} w_{2}$ & $\delta_{12} w_{1}$ \\
\hline$w_{1}$ & $w_{1}$ & 0 & 0 & 0 & 0 & $w_{2}$ & $w_{3}$ & 0 \\
\hline$w_{2}$ & $w_{2}$ & $w_{2}$ & $w_{1}$ & 0 & 0 & 0 & 0 & $w_{3}$ \\
\hline$w_{3}$ & 0 & $w_{3}$ & 0 & $w_{1}$ & $w_{2}$ & 0 & 0 & 0 \\
\hline$a_{1}$ & $a_{1}$ & 0 & $a_{2}+\delta_{13} w_{2}$ & $a_{3}+\delta_{14} w_{3}$ & $\tau_{1} v+\tau_{2} w$ & $\delta_{15} w_{2}$ & $\delta_{16} w_{3}$ & $\tau_{3} v+\tau_{4} w$ \\
\hline$a_{2}$ & $a_{2}$ & $a_{2}$ & $\delta_{17} w_{1}$ & $\tau_{5} v+\tau_{6} w$ & $a_{3}+\delta_{18} w_{3}$ & $a_{1}+\delta_{19} w_{1}$ & $\tau_{7} v+\tau_{8} w$ & $\delta_{20} w_{3}$ \\
\hline$a_{3}$ & 0 & $a_{3}$ & $\tau_{9} v+\tau_{10} w$ & $\delta_{21} w_{1}$ & $\delta_{22} w_{2}$ & $\tau_{11} v+\tau_{12} w$ & $a_{1}+\delta_{23} w_{1}$ & $a_{2}+\delta_{24} w_{2}$ \\
\hline
\end{tabular}

Tabela 3.38: $O \mathfrak{s l}_{3}(k)$-2-módulo $U^{41}:=\left[V_{3} \wedge\left(V_{0} \wedge V_{0}\right)\right] \curlywedge V_{3}^{*}$

Da tabela anterior temos que:

$$
U^{41} \simeq\left[V_{3} \curlywedge\left(V_{0} \wedge V_{0}\right)\right] \oplus V_{3}^{*} \Longleftrightarrow \delta_{i}=0 .
$$


22. Tabela para o $\mathfrak{s l}_{3}(k)-2$-módulo $U^{42}:=\left[\left(V_{0} \curlywedge V_{0}\right) \curlywedge V_{3}\right] \curlywedge V_{3}^{*}$

\begin{tabular}{|c||c|c|c|c|c|c|c|c|}
\hline & $h_{1}$ & $h_{2}$ & $e_{12}$ & $e_{13}$ & $e_{23}$ & $e_{21}$ & $e_{31}$ \\
\hline \hline$v$ & 0 & 0 & $\vartheta_{1} a_{3}+\delta_{19} w_{3}$ & $\eta_{1} a_{2}+\delta_{20} w_{2}$ & $\eta_{1} a_{1}+\delta_{21} w_{1}$ & $\iota_{1} a_{3}+\delta_{22} w_{3}$ & $\iota_{1} a_{2}+\delta_{23} w_{2}$ & $\vartheta_{1} a_{1}+\delta_{24} w_{1}$ \\
\hline$w$ & 0 & 0 & $\vartheta a_{3}+\delta_{13} w_{3}$ & $\eta a_{2}+\delta_{14} w_{2}$ & $\eta a_{1}+\delta_{15} w_{1}$ & $\iota a_{3}+\delta_{16} w_{3}$ & $\iota a_{2}+\delta_{17} w_{2}$ & $\vartheta a_{1}+\delta_{18} w_{1}$ \\
\hline$w_{1}$ & $w_{1}$ & 0 & 0 & 0 & 0 & $w_{2}$ & $w_{3}$ & 0 \\
\hline$w_{2}$ & $w_{2}$ & $w_{2}$ & $w_{1}$ & 0 & 0 & 0 & 0 & 0 \\
\hline$w_{3}$ & 0 & $w_{3}$ & 0 & $w_{1}$ & $w_{2}$ & 0 & $\delta_{4} w_{3}$ & 0 \\
\hline$a_{1}$ & $a_{1}$ & 0 & $a_{2}+\delta_{1} w_{2}$ & $a_{3}+\delta_{2} w_{3}$ & 0 & $\delta_{3} w_{2}$ & $\delta_{8} w_{3}$ \\
\hline$a_{2}$ & $a_{2}$ & $a_{2}$ & $\delta_{5} w_{1}$ & 0 & $a_{3}+\delta_{6} w_{3}$ & $a_{1}+\delta_{7} w_{1}$ & 0 \\
\hline$a_{3}$ & 0 & $a_{3}$ & 0 & $\delta_{9} w_{1}$ & $\delta_{10} w_{2}$ & 0 & $a_{1}+\delta_{11} w_{1}$ & $a_{2}+\delta_{12} w_{2}$ \\
\hline
\end{tabular}

Tabela 3.39: $O \mathfrak{s l}_{3}(k)-2$-módulo $U^{42}:=\left[\left(V_{0} \wedge V_{0}\right) \curlywedge V_{3}\right] \curlywedge V_{3}^{*}$

Da tabela anterior temos que:

$$
U^{42} \simeq\left[\left(V_{0}<V_{0}\right) \oplus V_{3}\right] \oplus V_{3}^{*} \Longleftrightarrow \delta_{i}=\eta=\vartheta=\iota=\eta_{1}=\vartheta_{1}=\iota_{1}=0 .
$$

23. Tabela para o $\mathfrak{s l}_{3}(k)-2-$ módulo $U^{45}:=\left[\left(V_{3} \curlywedge V_{0}\right) \curlywedge V_{0}\right] \curlywedge V_{3}^{*}$

\begin{tabular}{|c||c|c|c|c|c|c|c|c|}
\hline & $h_{1}$ & $h_{2}$ & $e_{12}$ & $e_{13}$ & $e_{23}$ & $e_{21}$ & $e_{31}$ & $e_{32}$ \\
\hline \hline$v$ & 0 & 0 & $\delta_{1} w_{3}$ & $\delta_{2} w_{2}$ & $\delta_{3} w_{1}$ & $\delta_{4} w_{3}$ & $\delta_{5} w_{2}$ & $\delta_{6} w_{1}$ \\
\hline$w$ & 0 & 0 & $\delta_{7} w_{3}$ & $\delta_{8} w_{2}$ & $\delta_{9} w_{1}$ & $\delta_{10} w_{3}$ & $\delta_{11} w_{2}$ & $\delta_{12} w_{1}$ \\
\hline$w_{1}$ & $w_{1}$ & 0 & 0 & 0 & 0 & $w_{2}$ & $w_{3}$ & 0 \\
\hline$w_{2}$ & $w_{2}$ & $w_{2}$ & $w_{1}$ & 0 & 0 & 0 & 0 & $w_{3}$ \\
\hline$w_{3}$ & 0 & $w_{3}$ & 0 & $w_{1}$ & $w_{2}$ & 0 & 0 & 0 \\
\hline$a_{1}$ & $a_{1}$ & 0 & $a_{2}+\delta_{13} w_{2}$ & $a_{3}+\delta_{14} w_{3}$ & $\beta w+\mu_{1} v$ & $\delta_{15} w_{2}$ & $\delta_{16} w_{3}$ & $\kappa w+\mu_{2} v$ \\
\hline$a_{2}$ & $a_{2}$ & $a_{2}$ & $\delta_{17} w_{1}$ & $\gamma w+\mu_{3} v$ & $a_{3}+\delta_{18} w_{3}$ & $a_{1}+\delta_{19} w_{1}$ & $\kappa w+\mu_{4} v$ & $\delta_{20} w_{3}$ \\
\hline$a_{3}$ & 0 & $a_{3}$ & $\gamma w+\mu_{5} v$ & $\delta_{21} w_{1}$ & $\delta_{22} w_{2}$ & $\beta w+\mu_{6} v$ & $a_{1}+\delta_{23} w_{1}$ & $a_{2}+\delta_{24} w_{2}$ \\
\hline
\end{tabular}

Tabela 3.40: $O \mathfrak{s l}_{3}(k)$-2-módulo $U^{45}:=\left[\left(V_{3} \curlywedge V_{0}\right) \curlywedge V_{0}\right] \curlywedge V_{3}^{*}$

Da tabela:

$$
U^{45} \simeq\left[\left(V_{3} \curlywedge V_{0}\right) \oplus V_{0}\right] \oplus V_{3}^{*} \Longleftrightarrow \delta_{i}=\mu_{j}=0 .
$$


24. Tabela para o $\mathfrak{s l}_{3}(k)-2$-módulo $U^{46}:=\left[V_{0} \curlywedge\left(V_{3} \wedge V_{0}\right)\right] \curlywedge V_{3}^{*}$

\begin{tabular}{|c|c|c|c|c|c|c|c|c|}
\hline & $h_{1}$ & $h_{2}$ & $e_{12}$ & $e_{13}$ & $e_{23}$ & $e_{21}$ & $e_{31}$ & $e_{32}$ \\
\hline$v$ & 0 & 0 & $\delta_{1} w_{3}$ & $\delta_{2} w_{2}$ & $\delta_{3} w_{1}$ & $\delta_{4} w_{3}$ & $\delta_{5} w_{2}$ & $\delta_{6} w_{1}$ \\
\hline$w$ & 0 & 0 & $\psi a_{3}+\delta_{7} w_{3}$ & $\varpi a_{2}+\delta_{8} w_{2}$ & $\varpi a_{1}+\delta_{9} w_{1}$ & $\varsigma a_{3}+\delta_{10} w_{3}$ & $\varsigma a_{2}+\delta_{11} w_{2}$ & $\psi a_{1}+\delta_{12} w_{1}$ \\
\hline$w_{1}$ & $w_{1}$ & 0 & 0 & 0 & 0 & $w_{2}$ & $w_{3}$ & 0 \\
\hline$w_{2}$ & $w_{2}$ & $w_{2}$ & $w_{1}$ & 0 & 0 & 0 & 0 & $w_{3}$ \\
\hline$w_{3}$ & 0 & $w_{3}$ & 0 & $w_{1}$ & $w_{2}$ & 0 & 0 & 0 \\
\hline$a_{1}$ & $a_{1}$ & 0 & $a_{2}+\delta_{13} w_{2}$ & $a_{3}+\delta_{14} w_{3}$ & $\beta v$ & $\delta_{15} w_{2}$ & $\delta_{16} w_{3}$ & $\kappa v$ \\
\hline$a_{2}$ & $a_{2}$ & $a_{2}$ & $\delta_{17} w_{1}$ & $\gamma v$ & $a_{3}+\delta_{18} w_{3}$ & $a_{1}+\delta_{19} w_{1}$ & $\kappa v$ & $\delta_{20} w_{3}$ \\
\hline$a_{3}$ & 0 & $a_{3}$ & $\gamma v$ & $\delta_{21} w_{1}$ & $\delta_{22} w_{2}$ & $\beta v$ & $a_{1}+\delta_{23} w_{1}$ & $a_{2}+\delta_{24} w_{2}$ \\
\hline
\end{tabular}

Tabela 3.41: $O \mathfrak{s l}_{3}(k)$-2-módulo $U^{46}:=\left[V_{0} \wedge\left(V_{3}<V_{0}\right)\right] \curlywedge V_{3}^{*}$

Da tabela:

$$
U^{46} \simeq\left[V_{0} \oplus\left(V_{3}<V_{0}\right)\right] \oplus V_{3}^{*} \Longleftrightarrow \delta_{i}=\varpi=\psi=\varsigma=0 .
$$

25. Tabela para o $\mathfrak{s l}_{3}(k)-2-$ módulo $U^{47}:=\left[\left(V_{0} \wedge V_{3}\right) \curlywedge V_{0}\right] \curlywedge V_{3}^{*}$

\begin{tabular}{|c||c|c|c|c|c|c|c|c|}
\hline & $h_{1}$ & $h_{2}$ & $e_{12}$ & $e_{13}$ & $e_{23}$ & $e_{21}$ & $e_{31}$ & $e_{32}$ \\
\hline \hline$v$ & 0 & 0 & $\delta_{1} w_{3}$ & $\delta_{2} w_{2}$ & $\delta_{3} w_{1}$ & $\delta_{4} w_{3}$ & $\delta_{5} w_{2}$ & $\delta_{6} w_{1}$ \\
\hline$w$ & 0 & 0 & $\omega a_{3}+\delta_{7} w_{3}$ & $\theta a_{2}+\delta_{8} w_{2}$ & $\theta a_{1}+\delta_{9} w_{1}$ & $\sigma a_{3}+\delta_{10} w_{3}$ & $\sigma a_{2}+\delta_{11} w_{2}$ & $\omega a_{1}+\delta_{12} w_{1}$ \\
\hline$w_{1}$ & $w_{1}$ & 0 & 0 & 0 & 0 & $w_{2}$ & $w_{3}$ & 0 \\
\hline$w_{2}$ & $w_{2}$ & $w_{2}$ & $w_{1}$ & 0 & 0 & 0 & 0 & 0 \\
\hline$w_{3}$ & 0 & $w_{3}$ & 0 & $w_{1}$ & $w_{2}$ & 0 & $w_{3}$ & 0 \\
\hline$a_{1}$ & $a_{1}$ & 0 & $a_{2}+\delta_{13} w_{2}$ & $a_{3}+\delta_{14} w_{3}$ & $\lambda_{1} v$ & $\delta_{15} w_{2}$ & $\delta_{16} w_{3}$ & $\lambda_{2} v$ \\
\hline$a_{2}$ & $a_{2}$ & $a_{2}$ & $\delta_{17} w_{1}$ & $\lambda_{3} v$ & $a_{3}+\delta_{18} w_{3}$ & $a_{1}+\delta_{19} w_{1}$ & $\lambda_{4} v$ & $\delta_{20} w_{3}$ \\
\hline$a_{3}$ & 0 & $a_{3}$ & $\lambda_{5} v$ & $\delta_{21} w_{1}$ & $\delta_{22} w_{2}$ & $\lambda_{6} v$ & $a_{1}+\delta_{23} w_{1}$ & $a_{2}+\delta_{24} w_{2}$ \\
\hline
\end{tabular}

Tabela 3.42: $O \mathfrak{s l}_{3}(k)-2$-módulo $U^{47}:=\left[\left(V_{0} \wedge V_{3}\right) \wedge V_{0}\right] \curlywedge V_{3}^{*}$

Da tabela anterior temos que:

$$
U^{47} \simeq\left[\left(V_{0} \oplus V_{3}\right) \oplus V_{0}\right] \oplus V_{3}^{*} \Longleftrightarrow \delta_{i}=\lambda_{j}=\theta=\eta=\iota=0 .
$$


26. Tabela para o $\mathfrak{s l}_{3}(k)-2$-módulo $U^{48}:=\left[V_{0} \curlywedge\left(V_{0} \wedge V_{3}\right)\right] \curlywedge V_{3}^{*}$

\begin{tabular}{|c||c|c|c|c|c|c|c|c|}
\hline & $h_{1}$ & $h_{2}$ & $e_{12}$ & $e_{13}$ & $e_{23}$ & $e_{21}$ & $e_{31}$ & $e_{32}$ \\
\hline \hline$v$ & 0 & 0 & $\omega a_{3}+\delta_{1} w_{3}$ & $\theta a_{2}+\delta_{2} w_{2}$ & $\theta a_{1}+\delta_{3} w_{1}$ & $\sigma a_{3}+\delta_{4} w_{3}$ & $\sigma a_{2}+\delta_{5} w_{2}$ & $\omega a_{1}+\delta_{6} w_{1}$ \\
\hline$w$ & 0 & 0 & $\psi a_{3}+\delta_{7} w_{3}$ & $\varpi a_{2}+\delta_{8} w_{2}$ & $\varpi a_{1}+\delta_{9} w_{1}$ & $\varsigma a_{3}+\delta_{10} w_{3}$ & $\varsigma a_{2}+\delta_{11} w_{2}$ & $\psi a_{1}+\delta_{12} w_{1}$ \\
\hline$w_{1}$ & $w_{1}$ & 0 & 0 & 0 & 0 & $w_{2}$ & $w_{3}$ & 0 \\
\hline$w_{2}$ & $w_{2}$ & $w_{2}$ & $w_{1}$ & 0 & 0 & 0 & 0 & 0 \\
\hline$w_{3}$ & 0 & $w_{3}$ & 0 & $w_{1}$ & $w_{2}$ & 0 & $\delta_{16} w_{3}$ & 0 \\
\hline$a_{1}$ & $a_{1}$ & 0 & $a_{2}+\delta_{13} w_{2}$ & $a_{3}+\delta_{14} w_{3}$ & 0 & $\delta_{15} w_{2}$ & $\delta_{1}$ & 0 \\
\hline$a_{2}$ & $a_{2}$ & $a_{2}$ & $\delta_{17} w_{1}$ & 0 & $a_{3}+\delta_{18} w_{3}$ & $a_{1}+\delta_{19} w_{1}$ & $\delta_{20} w_{3}$ \\
\hline$a_{3}$ & 0 & $a_{3}$ & 0 & $\delta_{21} w_{1}$ & $\delta_{22} w_{2}$ & 0 & $a_{1}+\delta_{23} w_{1}$ & $a_{2}+\delta_{24} w_{2}$ \\
\hline
\end{tabular}

Tabela 3.43: $O \mathfrak{s l}_{3}(k)-2$-módulo $U^{48}:=\left[V_{0} \curlywedge\left(V_{0} \wedge V_{3}\right)\right] \curlywedge V_{3}^{*}$

Da tabela anterior temos que:

$$
U^{48} \simeq\left[V_{0} \oplus\left(V_{0} \oplus V_{3}\right)\right] \oplus V_{3}^{*} \Longleftrightarrow \delta_{i}=\omega=\theta=\sigma=\psi=\varpi=\varsigma=\theta=\eta=\iota=0 .
$$

27. Tabela para o $\mathfrak{s l}_{3}(k)-2-$ módulo $U^{53}:=V_{3}^{*} \curlywedge\left[V_{3} \curlywedge\left(V_{0} \curlywedge V_{0}\right)\right]$

Com ajuda das tabelas 3.3 e 3.8 temos que a ação de $\mathfrak{s l}_{3}(k)$ sobre a base $\left\{x, y, x_{1}, x_{2}, x_{3}, y_{1}, y_{2}, y_{3}\right\}$ de $U^{35}$ é dada na tabela seguinte:

\begin{tabular}{|c||c|c|c|c|c|c|c|c|}
\hline & $h_{1}$ & $h_{2}$ & $e_{12}$ & $e_{13}$ & $e_{23}$ & $e_{21}$ & $e_{31}$ & $e_{32}$ \\
\hline \hline$x$ & 0 & 0 & 0 & 0 & 0 & 0 & 0 & 0 \\
\hline$y$ & 0 & 0 & 0 & 0 & 0 & 0 & 0 & 0 \\
\hline$x_{1}$ & $x_{1}$ & 0 & $x_{2}$ & $x_{3}$ & $\tau_{1} x+\tau_{2} y$ & 0 & 0 & $\tau_{3} x+\tau_{4} y$ \\
\hline$x_{2}$ & $x_{2}$ & $x_{2}$ & 0 & $\tau_{5} x+\tau_{6} y$ & $x_{3}$ & $x_{1}$ & $\tau_{7} x+\tau_{8} y$ & 0 \\
\hline$x_{3}$ & 0 & $x_{3}$ & $\tau_{9} x+\tau_{10} y$ & 0 & 0 & $\tau_{11} x+\tau_{12} y$ & $x_{1}$ & $x_{2}$ \\
\hline$y_{1}$ & $y_{1}$ & 0 & $\delta_{1} x_{2}$ & $\delta_{2} x_{3}$ & $\delta_{3} x+\delta_{4} y$ & $y_{2}+\delta_{5} x_{2}$ & $y_{3}+\delta_{6} x_{3}$ & $\delta_{7} x+\delta_{8} y$ \\
\hline$y_{2}$ & $y_{2}$ & $y_{2}$ & $y_{1}+\delta_{9} x_{1}$ & $\delta_{10} x+\delta_{11} y$ & $\delta_{12} x_{3}$ & $\delta_{13} x_{1}$ & $\delta_{14} x+\delta_{15} y$ & $y_{3}+\delta_{16} x_{3}$ \\
\hline$y_{3}$ & 0 & $y_{3}$ & $\delta_{17} x+\delta_{18} y$ & $y_{1}+\delta_{19} x_{1}$ & $y_{2}+\delta_{20} x_{2}$ & $\delta_{21} x+\delta_{22} y$ & $\delta_{23} x_{1}$ & $\delta_{24} x_{2}$ \\
\hline
\end{tabular}

Tabela 3.44: $O \mathfrak{s l}_{3}(k)-2-m o ́ d u l o ~ U^{53}:=V_{3}^{*} \wedge\left[V_{3} \wedge\left(V_{0} \wedge V_{0}\right)\right]$

Da tabela anterior temos que:

$$
U^{53} \simeq V_{3}^{*} \oplus\left[V_{3} \curlywedge\left(V_{0} \curlywedge V_{0}\right)\right] \Longleftrightarrow \delta_{i}=0 .
$$

Nesse caso, a tabela anterior fica assim: 


\begin{tabular}{|c||c|c|c|c|c|c|c|c|}
\hline & $h_{1}$ & $h_{2}$ & $e_{12}$ & $e_{13}$ & $e_{23}$ & $e_{21}$ & $e_{31}$ & $e_{32}$ \\
\hline \hline$x$ & 0 & 0 & 0 & 0 & 0 & 0 & 0 & 0 \\
\hline$y$ & 0 & 0 & 0 & 0 & 0 & 0 & 0 & 0 \\
\hline$x_{1}$ & $x_{1}$ & 0 & $x_{2}$ & $x_{3}$ & $\tau_{1} x+\tau_{2} y$ & 0 & 0 & $\tau_{3} x+\tau_{4} y$ \\
\hline$x_{2}$ & $x_{2}$ & $x_{2}$ & 0 & $\tau_{5} x+\tau_{6} y$ & $x_{3}$ & $x_{1}$ & $\tau_{7} x+\tau_{8} y$ & 0 \\
\hline$x_{3}$ & 0 & $x_{3}$ & $\tau_{9} x+\tau_{10} y$ & 0 & 0 & $\tau_{11} x+\tau_{12} y$ & $x_{1}$ & $x_{2}$ \\
\hline$y_{1}$ & $y_{1}$ & 0 & 0 & 0 & 0 & $y_{2}$ & $y_{3}$ & 0 \\
\hline$y_{2}$ & $y_{2}$ & $y_{2}$ & $y_{1}$ & 0 & 0 & 0 & 0 & $y_{3}$ \\
\hline$y_{3}$ & 0 & $y_{3}$ & 0 & $y_{1}$ & $y_{2}$ & 0 & 0 & 0 \\
\hline
\end{tabular}

Tabela 3.45: $O \mathfrak{s l}_{3}(k)-2$-módulo $U^{53}:=V_{3}^{*} \oplus\left[V_{3} \wedge\left(V_{0} \curlywedge V_{0}\right)\right]$

28. Tabela para o $\mathfrak{s l}_{3}(k)-2-$ módulo $U^{54}:=V_{3}^{*} \wedge\left[\left(V_{0} \wedge V_{0}\right) \prec V_{3}\right]$

\begin{tabular}{|c||c|c|c|c|c|c|c|c|}
\hline & $h_{1}$ & $h_{2}$ & $e_{12}$ & $e_{13}$ & $e_{23}$ & $e_{21}$ & $e_{31}$ & $e_{32}$ \\
\hline \hline$a$ & 0 & 0 & $\vartheta v_{3}$ & $\eta v_{2}$ & $\eta v_{1}$ & $\iota v_{3}$ & $\iota v_{2}$ & $\vartheta_{1}$ \\
\hline$b$ & 0 & 0 & $\vartheta_{1} v_{3}$ & $\eta_{1} v_{2}$ & $\eta_{1} v_{1}$ & $\iota_{1} v_{3}$ & $\iota_{1} v_{2}$ & $\vartheta_{1} v_{1}$ \\
\hline$v_{1}$ & $v_{1}$ & 0 & $v_{2}$ & $v_{3}$ & 0 & 0 & 0 & 0 \\
\hline$v_{2}$ & $v_{2}$ & $v_{2}$ & 0 & 0 & $v_{3}$ & $v_{1}$ & 0 & 0 \\
\hline$v_{3}$ & 0 & $v_{3}$ & 0 & 0 & 0 & 0 & $v_{1}$ & $v_{2}$ \\
\hline$y_{1}$ & $y_{1}$ & 0 & $\delta_{1} v_{2}$ & $\delta_{2} v_{3}$ & $\delta_{3} a+\delta_{4} b$ & $y_{2}+\delta_{5} v_{2}$ & $y_{3}+\delta_{6} v_{3}$ & $\delta_{7} a+\delta_{8} b$ \\
\hline$y_{2}$ & $y_{2}$ & $y_{2}$ & $y_{1}+\delta_{9} v_{1}$ & $\delta_{10} a+\delta_{11} b$ & $\delta_{12} v_{3}$ & $\delta_{13} v_{1}$ & $\delta_{14} a+\delta_{15} b$ & $y_{3}+\delta_{16} v_{3}$ \\
\hline$y_{3}$ & 0 & $y_{3}$ & $\delta_{17} a+\delta_{18} b$ & $y_{1}+\delta_{19} v_{1}$ & $y_{2}+\delta_{20} v_{2}$ & $\delta_{21} a+\delta_{22} b$ & $\delta_{23} v_{1}$ & $\delta_{24} v_{2}$ \\
\hline
\end{tabular}

Tabela 3.46: $O \mathfrak{s l}_{3}(k)-2$-módulo $U^{54}:=V_{3}^{*} \curlywedge\left[\left(V_{0} \curlywedge V_{0}\right) \curlywedge V_{3}\right]$

Da tabela anterior temos que:

$$
U^{54} \simeq V_{3}^{*} \oplus\left[\left(V_{0}<V_{0}\right) \oplus V_{3}\right] \Longleftrightarrow \delta_{i}=\vartheta=\iota=\eta=\vartheta_{1}=\iota_{1}=\eta_{1}=0 .
$$


29. Tabela para o $\mathfrak{s l}_{3}(k)-2$-módulo $U^{57}:=V_{3}^{*} \curlywedge\left[\left(V_{3} \wedge V_{0}\right) \curlywedge V_{0}\right]$

\begin{tabular}{|c||c|c|c|c|c|c|c|c|}
\hline & $h_{1}$ & $h_{2}$ & $e_{12}$ & $e_{13}$ & $e_{23}$ & $e_{21}$ & $e_{31}$ & $e_{32}$ \\
\hline \hline$v$ & 0 & 0 & 0 & 0 & 0 & 0 & 0 & 0 \\
\hline$j$ & 0 & 0 & 0 & 0 & 0 & 0 & 0 & 0 \\
\hline$j_{1}$ & $j_{1}$ & 0 & $j_{2}$ & $j_{3}$ & $\beta j+\mu_{1} v$ & 0 & 0 & $\kappa j+\mu_{2} v$ \\
\hline$j_{2}$ & $j_{2}$ & $j_{2}$ & 0 & $\gamma j+\mu_{3} v$ & $j_{3}$ & $j_{1}$ & $\kappa j+\mu_{4} v$ & 0 \\
\hline$j_{3}$ & 0 & $j_{3}$ & $\gamma j+\mu_{5} v$ & 0 & 0 & $\beta j+\mu_{6} v$ & $j_{1}$ & $j_{2}$ \\
\hline$y_{1}$ & $y_{1}$ & 0 & $\delta_{1} j_{2}$ & $\delta_{2} j_{3}$ & $\delta_{3} v+\delta_{4} j$ & $y_{2}+\delta_{5} j_{2}$ & $y_{3}+\delta_{6} j_{3}$ & $\delta_{7} v+\delta_{8} j$ \\
\hline$y_{2}$ & $y_{2}$ & $y_{2}$ & $y_{1}+\delta_{9} j_{1}$ & $\delta_{10} v+\delta_{11} j$ & $\delta_{12} j_{3}$ & $\delta_{13} j_{1}$ & $\delta_{14} v+\delta_{15} j$ & $y_{3}+\delta_{16} j_{3}$ \\
\hline$y_{3}$ & 0 & $y_{3}$ & $\delta_{17} v+\delta_{18} j$ & $y_{1}+\delta_{19} j_{1}$ & $y_{2}+\delta_{20} j_{2}$ & $\delta_{21} v+\delta_{22} j$ & $\delta_{23} j_{1}$ & $\delta_{24} j_{2}$ \\
\hline
\end{tabular}

Tabela 3.47: $O \mathfrak{s l}_{3}(k)-2$-módulo $U^{57}:=V_{3}^{*} \curlywedge\left[\left(V_{3} \wedge V_{0}\right) \curlywedge V_{0}\right]$

Da tabela anterior temos que:

$$
U^{57} \simeq V_{3}^{*} \oplus\left[\left(V_{3} \curlywedge V_{0}\right) \curlywedge V_{0}\right] \Longleftrightarrow \delta_{i}=0
$$

30. Tabela para o $\mathfrak{s l}_{3}(k)-2$-módulo $U^{58}:=V_{3}^{*} \curlywedge\left[V_{0} \curlywedge\left(V_{3} \curlywedge V_{0}\right)\right]$

\begin{tabular}{|c||c|c|c|c|c|c|c|c|}
\hline & $h_{1}$ & $h_{2}$ & $e_{12}$ & $e_{13}$ & $e_{23}$ & $e_{21}$ & $e_{31}$ & $e_{32}$ \\
\hline \hline$v$ & 0 & 0 & $\psi a_{3}$ & $\varpi a_{2}$ & $\varpi a_{1}$ & $\varsigma a_{3}$ & $\varsigma a_{2}$ & $\psi a_{3}$ \\
\hline$a$ & 0 & 0 & 0 & 0 & 0 & 0 & 0 & 0 \\
\hline$a_{1}$ & $a_{1}$ & 0 & $a_{2}$ & $a_{3}$ & $\beta a$ & 0 & 0 & $\kappa j$ \\
\hline$a_{2}$ & $a_{2}$ & $a_{2}$ & 0 & $\gamma a$ & $a_{3}$ & $a_{1}$ & $\kappa a$ & 0 \\
\hline$a_{3}$ & 0 & $a_{3}$ & $\gamma a$ & 0 & 0 & $\beta a$ & $a_{1}$ & $a_{2}$ \\
\hline$y_{1}$ & $y_{1}$ & 0 & $\delta_{1} a_{2}$ & $\delta_{2} a_{3}$ & $\delta_{3} v+\delta_{4} a$ & $y_{2}+\delta_{5} a_{2}$ & $y_{3}+\delta_{6} a_{3}$ & $\delta_{7} v+\delta_{8} a$ \\
\hline$y_{2}$ & $y_{2}$ & $y_{2}$ & $y_{1}+\delta_{9} a_{1}$ & $\delta_{10} v+\delta_{11} a$ & $\delta_{12} a_{3}$ & $\delta_{13} a_{1}$ & $\delta_{14} v+\delta_{15} a$ & $y_{3}+\delta_{16} j_{3}$ \\
\hline$y_{3}$ & 0 & $y_{3}$ & $\delta_{17} v+\delta_{18} a$ & $y_{1}+\delta_{19} a_{1}$ & $y_{2}+\delta_{20} a_{2}$ & $\delta_{21} v+\delta_{22} a$ & $\delta_{23} a_{1}$ & $\delta_{24} a_{2}$ \\
\hline
\end{tabular}

Tabela 3.48: $O \mathfrak{s l}_{3}(k)-2-m o ́ d u l o ~ U^{58}:=V_{3}^{*} \wedge\left[V_{0} \wedge\left(V_{3} \wedge V_{0}\right)\right]$

Da tabela anterior temos que:

$$
U^{58} \simeq V_{3}^{*} \oplus\left[V_{0} \oplus\left(V_{3} \curlywedge V_{0}\right)\right] \Longleftrightarrow \delta_{i}=\varpi=\psi=\varsigma=0
$$


31. Tabela para o $\mathfrak{s l}_{3}(k)-2$-módulo $U^{59}:=V_{3}^{*} \curlywedge\left[\left(V_{3} \curlywedge V_{0}\right) \curlywedge V_{0}\right]$

\begin{tabular}{|c||c|c|c|c|c|c|c|c|}
\hline & $h_{1}$ & $h_{2}$ & $e_{12}$ & $e_{13}$ & $e_{23}$ & $e_{21}$ & $e_{31}$ & $e_{32}$ \\
\hline \hline$a$ & 0 & 0 & 0 & 0 & 0 & 0 & 0 & 0 \\
\hline$j$ & 0 & 0 & $\omega j_{3}$ & $\theta j_{2}$ & $\theta j_{1}$ & $\sigma j_{3}$ & $\sigma j_{2}$ & $\omega j_{1}$ \\
\hline$j_{1}$ & $j_{1}$ & 0 & $j_{2}$ & $j_{3}$ & $\lambda_{1} a$ & 0 & 0 & $\lambda_{2} a$ \\
\hline$j_{2}$ & $j_{2}$ & $j_{2}$ & 0 & $\lambda_{3} a$ & $j_{3}$ & $j_{1}$ & $\lambda_{4} a$ & 0 \\
\hline$j_{3}$ & 0 & $j_{3}$ & $\lambda_{5} a$ & 0 & 0 & $\lambda_{6} a$ & $j_{1}$ & $j_{2}$ \\
\hline$y_{1}$ & $y_{1}$ & 0 & $\delta_{1} j_{2}$ & $\delta_{2} j_{3}$ & $\delta_{3} a+\delta_{4} j$ & $y_{2}+\delta_{5} j_{2}$ & $y_{3}+\delta_{6} j_{3}$ & $\delta_{7} a+\delta_{8} j$ \\
\hline$y_{2}$ & $y_{2}$ & $y_{2}$ & $y_{1}+\delta_{9} j_{1}$ & $\delta_{10} a+\delta_{11} j$ & $\delta_{12} j_{3}$ & $\delta_{13} j_{1}$ & $\delta_{14} a+\delta_{15} j$ & $y_{3}+\delta_{16} j_{3}$ \\
\hline$y_{3}$ & 0 & $y_{3}$ & $\delta_{17} a+\delta_{18} j$ & $y_{1}+\delta_{19} j_{1}$ & $y_{2}+\delta_{20} j_{2}$ & $\delta_{21} a+\delta_{22} j$ & $\delta_{23} j_{1}$ & $\delta_{24} j_{2}$ \\
\hline
\end{tabular}

Tabela 3.49: $O \mathfrak{s l}_{3}(k)$-2-módulo $U^{59}:=V_{3}^{*} 人\left[\left(V_{0} \curlywedge V_{3}\right) \curlywedge V_{0}\right]$

Da tabela anterior temos que:

$$
U^{59} \simeq V_{3}^{*} \oplus\left[\left(V_{3} \wedge V_{0}\right) \oplus V_{0}\right] \Longleftrightarrow \delta_{i}=\lambda_{j}=0
$$

32. Tabela para o $\mathfrak{s l}_{3}(k)$-2-módulo $U^{60}:=V_{3}^{*} \curlywedge\left[V_{0} \curlywedge\left(V_{0} \curlywedge V_{3}\right)\right]$

\begin{tabular}{|c||c|c|c|c|c|c|c|c|}
\hline & $h_{1}$ & $h_{2}$ & $e_{12}$ & $e_{13}$ & $e_{23}$ & $e_{21}$ & $e_{31}$ & $e_{32}$ \\
\hline \hline$w$ & 0 & 0 & $\psi a_{3}$ & $\varpi v_{2}$ & $\varpi v_{1}$ & $\varsigma v_{3}$ & $\varsigma v_{2}$ & $\psi v_{3}$ \\
\hline$v$ & 0 & 0 & $\omega v_{3}$ & $\theta v_{2}$ & $\theta v_{1}$ & $\sigma v_{3}$ & $\sigma v_{2}$ & $\omega v_{1}$ \\
\hline$v_{1}$ & $v_{1}$ & 0 & $v_{2}$ & $v_{3}$ & 0 & 0 & 0 & 0 \\
\hline$v_{2}$ & $v_{2}$ & $v_{2}$ & 0 & 0 & $v_{3}$ & $v_{1}$ & 0 & 0 \\
\hline$v_{3}$ & 0 & $v_{3}$ & 0 & 0 & 0 & 0 & $v_{1}$ & $v_{2}$ \\
\hline$y_{1}$ & $y_{1}$ & 0 & $\delta_{1} v_{2}$ & $\delta_{2} v_{3}$ & $\delta_{3} v+\delta_{4} w$ & $y_{2}+\delta_{5} v_{2}$ & $y_{3}+\delta_{6} v_{3}$ & $\delta_{7} v+\delta_{8} w$ \\
\hline$y_{2}$ & $y_{2}$ & $y_{2}$ & $y_{1}+\delta_{9} v_{1}$ & $\delta_{10} v+\delta_{11} w$ & $\delta_{12} v_{3}$ & $\delta_{13} v_{1}$ & $\delta_{14} v+\delta_{15} w$ & $y_{3}+\delta_{16} j_{3}$ \\
\hline$y_{3}$ & 0 & $y_{3}$ & $\delta_{17} v+\delta_{18} w$ & $y_{1}+\delta_{19} v_{1}$ & $y_{2}+\delta_{20} v_{2}$ & $\delta_{21} v+\delta_{22} w$ & $\delta_{23} v_{1}$ & $\delta_{24} v_{2}$ \\
\hline
\end{tabular}

Tabela 3.50: $O \mathfrak{s l}_{3}(k)$-2-módulo $U^{60}:=V_{3}^{*} \curlywedge\left[V_{0} \curlywedge\left(V_{0} \wedge V_{3}\right)\right]$

Da tabela anterior temos que:

$$
U^{60} \simeq V_{3}^{*} \oplus\left[V_{0} \oplus\left(V_{0} \wedge V_{3}\right)\right] \Longleftrightarrow \delta_{i}=\varpi=\psi=\varsigma=\omega=\theta=\sigma=0
$$


33. Tabela para o $\mathfrak{s l}_{3}(k)-2$-módulo $U^{65}:=\left(V_{3}^{*} \curlywedge V_{3}\right) \curlywedge\left(V_{0} \wedge V_{0}\right)$

\begin{tabular}{|c||c|c|c|c|c|c|c|c|}
\hline & $h_{1}$ & $h_{2}$ & $e_{12}$ & $e_{13}$ & $e_{23}$ & $e_{21}$ & $e_{31}$ & $e_{32}$ \\
\hline \hline$v$ & 0 & 0 & 0 & 0 & 0 & 0 & 0 & 0 \\
\hline$w$ & 0 & 0 & 0 & 0 & 0 & 0 & 0 & 0 \\
\hline$v_{1}$ & $v_{1}$ & 0 & $v_{2}$ & $v_{3}$ & $\delta_{1} v+\delta_{2} w$ & 0 & 0 & $\delta_{3} v+\delta_{4} w$ \\
\hline$v_{2}$ & $v_{2}$ & $v_{2}$ & 0 & $\delta_{5} v+\delta_{6} w$ & $v_{3}$ & $v_{1}$ & $\delta_{7} v+\delta_{8} w$ & 0 \\
\hline$v_{3}$ & 0 & $v_{3}$ & $\delta_{9} v+\delta_{10} w$ & 0 & 0 & $\delta_{11} v+\delta_{12} w$ & $v_{1}$ & $v_{2}$ \\
\hline$u_{1}$ & $u_{1}$ & 0 & $\alpha v_{2}$ & $\alpha v_{3}$ & $\delta_{13} v+\delta_{14} w$ & $u_{2}+\gamma v_{2}$ & $u_{3}+\beta v_{3}$ & $\delta_{15} v+\delta_{16} w$ \\
\hline$u_{2}$ & $u_{2}$ & $u_{2}$ & $u_{1}+\alpha v_{1}$ & $\delta_{17} v+\delta_{18} w$ & $\gamma v_{3}$ & $\gamma v_{1}$ & $\delta_{19} v+\delta_{20} w$ & $u_{3}+\beta v_{3}$ \\
\hline$u_{3}$ & 0 & $u_{3}$ & $\delta_{21} v+\delta_{22} w$ & $u_{1}+\alpha v_{1}$ & $u_{2}+\gamma v_{2}$ & $\delta_{23} v+\delta_{24} w$ & $\beta v_{1}$ & $\beta v_{2}$ \\
\hline
\end{tabular}

Tabela 3.51: $O \mathfrak{s l}_{3}(k)-2$-módulo $U^{65}:=\left(V_{3}^{*} \curlywedge V_{3}\right) \curlywedge\left(V_{0} \curlywedge V_{0}\right)$

Da tabela anterior e de 3.2.4.1 temos que:

$$
U^{65} \simeq\left(V_{3}^{*} \oplus V_{3}\right) \oplus\left(V_{0} \oplus V_{0}\right) \Longleftrightarrow \delta_{i}=\alpha=\beta=\gamma=0 .
$$

34. Tabela para o $\mathfrak{s l}_{3}(k)-2$-módulo $U^{67}:=\left(V_{3} \curlywedge V_{3}^{*}\right) \curlywedge\left(V_{0} \curlywedge V_{0}\right)$

\begin{tabular}{|c||c|c|c|c|c|c|c|c|}
\hline & $h_{1}$ & $h_{2}$ & $e_{12}$ & $e_{13}$ & $e_{23}$ & $e_{21}$ & $e_{31}$ & $e_{32}$ \\
\hline \hline$v$ & 0 & 0 & 0 & 0 & 0 & 0 & 0 & 0 \\
\hline$w$ & 0 & 0 & 0 & 0 & 0 & 0 & 0 & 0 \\
\hline$w_{1}$ & $w_{1}$ & 0 & 0 & 0 & $\delta_{1} v+\delta_{2} w$ & $w_{2}$ & $w_{3}$ & 0 \\
\hline$w_{2}$ & $w_{2}$ & $w_{2}$ & $w_{1}$ & $\delta_{5} v+\delta_{6} w$ & 0 & 0 & $\delta_{7} v+\delta_{8} w$ & $w_{3}$ \\
\hline$w_{3}$ & 0 & $w_{3}$ & $\delta_{9} v+\delta_{10} w$ & $w_{1}$ & $w_{2}$ & $\delta_{11} v+\delta_{12} w$ & 0 & 0 \\
\hline$u_{1}$ & $u_{1}$ & 0 & $u_{2}+\alpha w_{2}$ & $u_{3}+\gamma w_{3}$ & $\delta_{13} v+\delta_{14 w} w$ & $\beta w_{2}$ & $\beta w_{3}$ & $\delta_{15} v+\delta_{16} w$ \\
\hline$u_{2}$ & $u_{2}$ & $u_{2}$ & $\alpha w_{1}$ & $\delta_{17} v+\delta_{18} w$ & $u_{3}+\gamma w_{3}$ & $u_{1}+\beta v_{1}$ & $\delta_{19} v+\delta_{20} w$ & $\alpha w_{3}$ \\
\hline$u_{3}$ & 0 & $u_{3}$ & $\delta_{21} v+\delta_{22} w$ & $\gamma w_{1}$ & $\gamma w_{2}$ & $\delta_{23} v+\delta_{24} w$ & $u_{1}+\beta w_{1}$ & $u_{2}+\alpha w_{2}$ \\
\hline
\end{tabular}

Tabela 3.52: $O \mathfrak{s l}_{3}(k)-2-m o ́ d u l o ~ U^{67}:=\left(V_{3} \wedge V_{3}^{*}\right) \wedge\left(V_{0} \wedge V_{0}\right)$

Da tabela anterior e de 3.2.4.3. temos que:

$$
U^{67} \simeq\left(V_{3}^{*} \oplus V_{3}\right) \oplus\left(V_{0} \oplus V_{0}\right) \Longleftrightarrow \delta_{i}=\alpha=\beta=\gamma=0 .
$$


35. Tabela para o $\mathfrak{s l}_{3}(k)-2-$ módulo $U^{101}:=\left(V_{0} \curlywedge V_{0}\right) \curlywedge\left(V_{3}^{*} \curlywedge V_{3}\right)$

\begin{tabular}{|c||c|c|c|c|c|c|c|c|}
\hline & $h_{1}$ & $h_{2}$ & $e_{12}$ & $e_{13}$ & $e_{23}$ & $e_{21}$ & $e_{31}$ & $e_{32}$ \\
\hline \hline$v_{1}$ & $v_{1}$ & 0 & $v_{2}$ & $v_{3}$ & 0 & 0 & 0 & 0 \\
\hline$v_{2}$ & $v_{2}$ & $v_{2}$ & 0 & 0 & $v_{3}$ & $v_{1}$ & 0 \\
\hline$v_{3}$ & 0 & $v_{3}$ & 0 & 0 & 0 & 0 & $v_{1}$ & $v_{2}$ \\
\hline$u_{1}$ & $u_{1}$ & 0 & $\alpha v_{2}$ & $\alpha v_{3}$ & 0 & $u_{2}+\gamma v_{2}$ & $u_{3}+\beta v_{3}$ & 0 \\
\hline$u_{2}$ & $u_{2}$ & $u_{2}$ & $u_{1}+\alpha v_{1}$ & 0 & $\gamma v_{3}$ & $u_{1}$ & 0 & $u_{3}+\beta v_{3}$ \\
\hline$u_{3}$ & 0 & $u_{3}$ & 0 & $u_{1}+\alpha v_{1}$ & $u_{2}+\gamma v_{2}$ & 0 & $\beta v_{1}$ & $\delta_{2}$ \\
\hline$u$ & 0 & 0 & $\delta_{1} v_{3}+\delta_{2} u_{3}$ & $\delta_{3} v_{2}+\delta_{4} u_{2}$ & $\delta_{5} v_{1}+\delta_{6} u_{1}$ & $\delta_{7} v_{3}+\delta_{8} u_{3}$ & $\delta_{9} v_{2}+\delta_{10} u_{2}$ & $\delta_{11} v_{1}+\delta_{12} u_{1}$ \\
\hline$v$ & 0 & 0 & $\delta_{13} v_{3}+\delta_{14} u_{3}$ & $\delta_{15} v_{2}+\delta_{16} u_{2}$ & $\delta_{17} v_{1}+\delta_{18} u_{1}$ & $\delta_{19} v_{3}+\delta_{20} u_{3}$ & $\delta_{21} v_{2}+\delta_{22} u_{2}$ & $\delta_{23} v_{1}+\delta_{24} u_{1}$ \\
\hline
\end{tabular}

Tabela 3.53: $O \mathfrak{s l}_{3}(k)$-2-módulos $U^{101}:=\left(V_{0} \curlywedge V_{0}\right) \curlywedge\left(V_{3}^{*} \curlywedge V_{3}\right)$

Da tabela anterior temos que:

$$
U^{101} \simeq\left(V_{0} \oplus V_{0}\right) \oplus\left(V_{3}^{*} \oplus V_{3}\right) \Longleftrightarrow \delta_{i}=\alpha=\beta=\gamma=0 .
$$

36. Tabela para o $\mathfrak{s l}_{3}(k)-2-$ módulo $U^{103}:=\left(V_{0} \curlywedge V_{0}\right) \curlywedge\left(V_{3} \curlywedge V_{3}^{*}\right)$

\begin{tabular}{|c||c|c|c|c|c|c|c|c|}
\hline & $h_{1}$ & $h_{2}$ & $e_{12}$ & $e_{13}$ & $e_{23}$ & $e_{21}$ & $e_{31}$ & $e_{32}$ \\
\hline \hline$w_{1}$ & $w_{1}$ & 0 & 0 & 0 & 0 & $v_{2}$ & $w_{3}$ & 0 \\
\hline$w_{2}$ & $w_{2}$ & $w_{2}$ & $w_{1}$ & 0 & 0 & 0 & 0 \\
\hline$w_{3}$ & 0 & $w_{3}$ & 0 & $w_{1}$ & $w_{2}$ & 0 & 0 \\
\hline$u_{1}$ & $u_{1}$ & 0 & $u_{2}+\alpha w_{2}$ & $u_{3}+\gamma w_{3}$ & 0 & $\beta w_{2}$ & $\beta w_{3}$ & 0 \\
\hline$u_{2}$ & $u_{2}$ & $u_{2}$ & $\alpha w_{1}$ & 0 & $u_{3}+\gamma w_{3}$ & $u_{1}+\beta w_{1}$ & 0 & 0 \\
\hline$u_{3}$ & 0 & $u_{3}$ & 0 & $\gamma w_{1}$ & $\gamma w_{2}$ & 0 & $u_{1}+\beta w_{1}$ & $u_{2}+\alpha w_{2}$ \\
\hline$w$ & 0 & 0 & $\delta_{1} w_{3}+\delta_{2} u_{3}$ & $\delta_{3} w_{2}+\delta_{4} u_{2}$ & $\delta_{5} w_{1}+\delta_{6} u_{1}$ & $\delta_{7} w_{3}+\delta_{8} u_{3}$ & $\delta_{9} w_{2}+\delta_{10} u_{2}$ & $\delta_{11} w_{1}+\delta_{12} u_{1}$ \\
\hline$u$ & 0 & 0 & $\delta_{13} w_{3}+\delta_{14} u_{3}$ & $\delta_{15} w_{2}+\delta_{16} u_{2}$ & $\delta_{17} w_{1}+\delta_{18} u_{1}$ & $\delta_{19} w_{3}+\delta_{20} u_{3}$ & $\delta_{21} w_{2}+\delta_{22} u_{2}$ & $\delta_{23} w_{1}+\delta_{24} u_{1}$ \\
\hline
\end{tabular}

Tabela 3.54: $O \mathfrak{s l}_{3}(k)-2$-módulos $U^{103}:=\left(V_{0} \wedge V_{0}\right) \curlywedge\left(V_{3} \wedge V_{3}^{*}\right)$

Da tabela anterior temos que:

$$
U^{103} \simeq\left(V_{0} \oplus V_{0}\right) \oplus\left(V_{3} \oplus V_{3}^{*}\right) \Longleftrightarrow \delta_{i}=\alpha=\beta=\gamma=0 .
$$


37. Tabela para o $\mathfrak{s l}_{3}(k)-2$-módulo $U^{138}:=\left[\left(V_{3} \wedge V_{0}\right) \wedge V_{3}^{*}\right] \curlywedge V_{0}$

\begin{tabular}{|c||c|c|c|c|c|c|c|c|}
\hline & $h_{1}$ & $h_{2}$ & $e_{12}$ & $e_{13}$ & $e_{23}$ & $e_{21}$ & $e_{31}$ & $e_{32}$ \\
\hline \hline$a$ & 0 & 0 & 0 & 0 & 0 & 0 & 0 & 0 \\
\hline$a_{1}$ & $a_{1}$ & 0 & 0 & 0 & $\lambda_{1} a$ & $a_{2}$ & $a_{3}$ & $\lambda_{2} a$ \\
\hline$a_{2}$ & $a_{2}$ & $a_{2}$ & $a_{1}$ & $\lambda_{3} a$ & 0 & 0 & $\lambda_{4} a$ & 0 \\
\hline$a_{3}$ & 0 & $a_{3}$ & $\lambda_{5} a$ & $a_{1}$ & $a_{2}$ & $\lambda_{6} a$ & 0 & 0 \\
\hline$b$ & 0 & 0 & $\delta_{1} a_{3}$ & $\delta_{2} a_{2}$ & $\delta_{3} a_{1}$ & $\delta_{4} a_{3}$ & $\delta_{5} a_{2}$ & $\delta_{6} a_{1}$ \\
\hline$b_{1}$ & $b_{1}$ & 0 & $b_{2}+\delta_{7} a_{2}$ & $b_{3}+\delta_{8} a_{3}$ & $\beta b+\lambda_{7} a$ & $\delta_{9} a_{2}$ & $\delta_{10} a_{3}$ & $\kappa b+\lambda_{8} a$ \\
\hline$b_{2}$ & $b_{2}$ & $b_{2}$ & $\delta_{11} a_{1}$ & $\gamma b+\lambda_{9} a$ & $b_{3}+\delta_{12} a_{3}$ & $b_{1}+\delta_{13} a_{1}$ & $\kappa b+\lambda_{10} a$ & $\delta_{14} a_{3}$ \\
\hline$b_{3}$ & 0 & $b_{3}$ & $\gamma b+\lambda_{11} a$ & $\delta_{15} a_{1}$ & $\delta_{16} a_{2}$ & $\beta b+\lambda_{12} a$ & $b_{1}+\delta_{17} a_{1}$ & $b_{2}+\delta_{18} a_{2}$ \\
\hline
\end{tabular}

Tabela 3.55: $O \mathfrak{s l}_{3}(k)-2$-módulos $U^{138}:=\left[\left(V_{3} \curlywedge V_{0}\right) \curlywedge V_{3}^{*}\right] \curlywedge V_{0}$

Da tabela anterior temos que:

$$
U^{138} \simeq\left[\left(V_{3} \oplus V_{0}\right) \oplus V_{3}^{*}\right] \oplus V_{0} \Longleftrightarrow \delta_{i}=\lambda_{j}=\kappa=\beta=\gamma=0 .
$$

38. Tabela para o $\mathfrak{s l}_{3}(k)-2-$ módulo $U^{139}:=\left[V_{3}^{*} \curlywedge\left(V_{3} \curlywedge V_{0}\right)\right] \curlywedge V_{0}$

\begin{tabular}{|c||c|c|c|c|c|c|c|c|}
\hline & $h_{1}$ & $h_{2}$ & $e_{12}$ & $e_{13}$ & $e_{23}$ & $e_{21}$ & $e_{31}$ & $e_{32}$ \\
\hline \hline$i$ & 0 & 0 & 0 & 0 & 0 & 0 & 0 & 0 \\
\hline$i_{1}$ & $i_{1}$ & 0 & $i_{2}$ & $i_{3}$ & $\beta i+\lambda_{1} a$ & 0 & 0 & $\kappa i+\lambda_{2} a$ \\
\hline$i_{2}$ & $i_{2}$ & $i_{2}$ & 0 & $\gamma i+\lambda_{3} a$ & $i_{3}$ & $i_{1}$ & $\kappa i+\lambda_{4} a$ & 0 \\
\hline$i_{3}$ & 0 & $i_{3}$ & $\gamma i+\lambda_{5} a$ & 0 & 0 & $\beta i+\lambda_{6} a$ & $i_{1}$ & $i_{2}$ \\
\hline$a_{1}$ & $a_{1}$ & 0 & $\delta_{1} i_{2}$ & $\delta_{2} i_{3}$ & $\delta_{3} i+\lambda a$ & $a_{2}+\delta_{4} i_{2}$ & $a_{3}+\delta_{5} i_{3}$ & $\delta_{6} i+\lambda_{8} a$ \\
\hline$a_{2}$ & $a_{2}$ & $a_{2}$ & $a_{1}+\delta_{7} i_{1}$ & $\delta_{8} i+\lambda_{9} a$ & $\delta_{9} i_{3}$ & $\delta_{10} i_{1}$ & $\delta_{11} i+\lambda_{10} a$ & $a_{3}+\delta_{12} i_{3}$ \\
\hline$a_{3}$ & 0 & $a_{3}$ & $\delta_{13} i+\lambda_{11} a$ & $a_{1}+\delta_{14} i_{1}$ & $a_{2}+\delta_{15} i_{2}$ & $\delta_{16} i+\lambda_{12} a$ & $\delta_{17} i_{1}$ & $\delta_{18} i_{2}$ \\
\hline$a$ & 0 & 0 & 0 & 0 & 0 & 0 & 0 & 0 \\
\hline
\end{tabular}

Tabela 3.56: $O \mathfrak{s l}_{3}(k)$-2-módulos $U^{139}:=\left[V_{3}^{*} \curlywedge\left(V_{3} \curlywedge V_{0}\right)\right] \curlywedge V_{0}$

Da tabela anterior temos que:

$$
U^{139} \simeq\left[V_{3}^{*} \oplus\left(V_{3} \oplus V_{0}\right)\right] \oplus V_{0} \Longleftrightarrow \delta_{i}=\lambda_{j}=\kappa=\beta=\gamma=0 .
$$


39. Tabela para o $\mathfrak{s l}_{3}(k)-2$-módulo $\left.U^{142}:=\left[\left(V_{0} \wedge V_{3}\right) \curlywedge V_{3}^{*}\right)\right] \curlywedge V_{0}$

\begin{tabular}{|c||c|c|c|c|c|c|c|c|}
\hline & $h_{1}$ & $h_{2}$ & $e_{12}$ & $e_{13}$ & $e_{23}$ & $e_{21}$ & $e_{31}$ & $e_{32}$ \\
\hline \hline$x$ & 0 & 0 & 0 & 0 & 0 & 0 & 0 & 0 \\
\hline$x_{1}$ & $x_{1}$ & 0 & 0 & 0 & $\lambda_{1} x$ & $x_{2}$ & $x_{3}$ & $\lambda_{2} x$ \\
\hline$x_{2}$ & $x_{2}$ & $x_{2}$ & $x_{1}$ & $\lambda_{3} x$ & 0 & 0 & $\lambda_{4} x$ & $x_{3}$ \\
\hline$x_{3}$ & 0 & $x_{3}$ & $\lambda_{5} x$ & $x_{1}$ & $x_{2}$ & $\lambda_{6} x$ & 0 & 0 \\
\hline$a_{1}$ & $a_{1}$ & 0 & $a_{2}+\delta_{7} x_{2}$ & $a_{3}+\delta_{8} x_{3}$ & $\lambda_{7} x$ & $\delta_{10} x_{2}$ & $\delta_{11} x_{3}$ & $\lambda_{8} x$ \\
\hline$a_{2}$ & $a_{2}$ & $a_{2}$ & $\delta_{7} x_{1}$ & $\lambda_{9} x$ & $a_{3}+\delta_{9} x_{3}$ & $a_{1}+\delta_{10} x_{1}$ & $\lambda_{10} x$ & $\delta_{12} x_{3}$ \\
\hline$a_{3}$ & 0 & $a_{3}$ & $\lambda_{11} x$ & $\delta_{8} x_{1}$ & $\delta_{9} x_{2}$ & $\lambda_{12} x$ & $a_{1}+\delta_{11} x_{1}$ & $a_{2}+\delta_{12} x_{2}$ \\
\hline$a$ & 0 & 0 & $\omega a_{3}+\delta_{1} x_{3}$ & $\theta a_{2}+\delta_{2} x_{2}$ & $\theta a_{1}+\delta_{3} x_{1}$ & $\sigma a_{3}+\delta_{4} x_{3}$ & $\sigma a_{2}+\delta_{5} x_{2}$ & $w a_{1}+\delta_{6} x_{1}$ \\
\hline
\end{tabular}

Tabela 3.57: $O \mathfrak{s l}_{3}(k)-2-$ módulos $U^{142}:=\left[\left(V_{0} \wedge V_{3}\right) \wedge V_{3}^{*}\right] \wedge V_{0}$

Da tabela anterior temos que:

$$
\left.U^{142} \simeq\left[\left(V_{0} \oplus V_{3}\right) \oplus V_{3}^{*}\right)\right] \oplus V_{0} \Longleftrightarrow \delta_{i}=\lambda_{j}=\omega=\theta=\sigma=0 .
$$

40. Tabela para o $\mathfrak{s l}_{3}(k)-2$-módulo $U^{143}:=\left[V_{3}^{*} \curlywedge\left(V_{0} \curlywedge V_{3}\right)\right] \curlywedge V_{0}$

\begin{tabular}{|c||c|c|c|c|c|c|c|c|}
\hline & $h_{1}$ & $h_{2}$ & $e_{12}$ & $e_{13}$ & $e_{23}$ & $e_{21}$ & $e_{31}$ & $e_{32}$ \\
\hline \hline$v$ & 0 & 0 & $\alpha v_{3}$ & $\xi v_{2}$ & $\xi v_{1}$ & $\beta v_{3}$ & $\beta v_{2}$ & $\alpha v_{1}$ \\
\hline$v_{1}$ & $v_{1}$ & 0 & $v_{2}$ & $v_{3}$ & $\lambda_{1} y$ & 0 & 0 & $\lambda_{2} y$ \\
\hline$v_{2}$ & $v_{2}$ & $v_{2}$ & 0 & $\lambda_{3} y$ & $v_{3}$ & $v_{1}$ & $\lambda_{4} y$ & 0 \\
\hline$v_{3}$ & 0 & $v_{3}$ & $\lambda_{5} y$ & 0 & 0 & $\lambda_{6} y$ & $v_{1}$ & $v_{2}$ \\
\hline$y_{1}$ & $y_{1}$ & 0 & $\delta_{1} v_{2}$ & $\delta_{2} v_{3}$ & $\delta_{3} v+\lambda_{7} y$ & $\delta_{4} v_{2}$ & $\delta_{5} v_{3}$ & $\delta_{6}+\lambda_{8} y$ \\
\hline$y_{2}$ & $y_{2}$ & $y_{2}$ & $y_{1}+\delta_{7} v_{1}$ & $\delta_{8} v+\lambda_{9} y$ & $\delta_{9} v_{3}$ & $\delta_{10} v_{1}$ & $\delta_{11} v+\lambda_{10} y$ & $y_{3}+\delta_{12} v_{3}$ \\
\hline$y_{3}$ & 0 & $y_{3}$ & $\delta_{13} v+\lambda_{11} y$ & $y_{1}+\delta_{14} v_{1}$ & $y_{2}+\delta_{15} v_{2}$ & $\delta_{16} v+\lambda_{12} y$ & $\delta_{17} v_{1}$ & $\delta_{18} v_{2}$ \\
\hline$y$ & 0 & 0 & 0 & 0 & 0 & 0 & 0 & 0 \\
\hline
\end{tabular}

Tabela 3.58: $O \mathfrak{s l}_{3}(k)-2$-módulos $U^{143}:=\left[V_{3}^{*} \curlywedge\left(V_{0} \curlywedge V_{3}\right)\right] \curlywedge V_{0}$

Da tabela anterior temos que:

$$
U^{143} \simeq\left[V_{3}^{*} \oplus\left(V_{0} \oplus V_{3}\right)\right] \oplus V_{0} \Longleftrightarrow \delta_{i}=\lambda_{j}=\alpha=\beta=\xi=0 .
$$


41. Tabela para o $\mathfrak{s l}_{3}(k)-2$-módulo $U^{144}:=\left[\left(V_{3}^{*} \curlywedge V_{0}\right) \wedge V_{3}\right] \curlywedge V_{0}$

\begin{tabular}{|c||c|c|c|c|c|c|c|c|}
\hline & $h_{1}$ & $h_{2}$ & $e_{12}$ & $e_{13}$ & $e_{23}$ & $e_{21}$ & $e_{31}$ & $e_{32}$ \\
\hline \hline$v$ & 0 & 0 & 0 & 0 & 0 & 0 & 0 & 0 \\
\hline$v_{1}$ & $v_{1}$ & 0 & $v_{2}$ & $v_{3}$ & $\lambda_{1} v$ & 0 & 0 & $\lambda_{2} v$ \\
\hline$v_{2}$ & $v_{2}$ & $v_{2}$ & 0 & $\lambda_{3} v$ & $v_{3}$ & $v_{1}$ & $\lambda_{4} v$ & 0 \\
\hline$v_{3}$ & 0 & $v_{3}$ & $\lambda_{5} v$ & 0 & 0 & $\lambda_{6} v$ & $v_{1}$ & $v_{2}$ \\
\hline$u_{1}$ & $u_{1}$ & 0 & $\delta_{7} v_{2}$ & $\delta_{8} v_{3}$ & $\pi u+\lambda_{1} v$ & $u_{2}+\delta_{9} v_{2}$ & $u_{3}+\delta_{10} v_{3}$ & $\lambda u+\lambda_{2} v$ \\
\hline$u_{2}$ & $u_{2}$ & $u_{2}$ & $u_{1}+\delta_{11} v_{1}$ & $\pi u+\lambda_{3} v$ & $\delta_{12} v_{3}$ & $\delta_{13} v_{1}$ & $\xi u+\lambda_{4} v$ & $u_{3}+\delta_{14} v_{3}$ \\
\hline$u_{3}$ & 0 & $u_{3}$ & $\lambda u+\lambda_{5} v$ & $u_{1}+\delta_{15} v_{1}$ & $u_{2}+\delta_{16} v_{2}$ & $\xi u+\lambda_{6} v$ & $\delta_{17} v_{1}$ & $\delta_{18} v_{2}$ \\
\hline$u$ & 0 & 0 & $\delta_{1} v_{3}$ & $\delta_{2} v_{2}$ & $\delta_{3} v_{1}$ & $\delta_{4} v_{3}$ & $\delta_{5} v_{2}$ & $\delta_{6} v_{1}$ \\
\hline
\end{tabular}

Tabela 3.59: $O \mathfrak{s l}_{3}(k)-2$-módulos $U^{144}:=\left[\left(V_{3}^{*} \curlywedge V_{0}\right) \curlywedge V_{3}\right] \curlywedge V_{0}$

Da tabela anterior temos que:

$$
U^{144} \simeq\left[\left(V_{3}^{*} \oplus V_{0}\right) \oplus V_{3}\right] \oplus V_{0} \Longleftrightarrow \delta_{i}=\lambda_{j}=\pi=\lambda=\xi=0 .
$$

42. Tabela para o $\mathfrak{s l}_{3}(k)-2$-módulo $U^{145}:=\left[V_{3} \curlywedge\left(V_{3}^{*} \curlywedge V_{0}\right)\right] \curlywedge V_{0}$

\begin{tabular}{|c||c|c|c|c|c|c|c|c|}
\hline & $h_{1}$ & $h_{2}$ & $e_{12}$ & $e_{13}$ & $e_{23}$ & $e_{21}$ & $e_{31}$ & $e_{32}$ \\
\hline \hline$u$ & 0 & 0 & 0 & 0 & 0 & 0 & 0 & 0 \\
\hline$u_{1}$ & $u_{1}$ & 0 & 0 & 0 & $\pi u+\lambda_{1} g$ & $u_{2}$ & $u_{3}$ & $\lambda u+\lambda_{2} g$ \\
\hline$u_{2}$ & $u_{2}$ & $u_{2}$ & $u_{1}$ & $\pi u+\lambda_{3} g$ & 0 & 0 & $\xi u+\lambda_{4} g$ & $u_{3}$ \\
\hline$u_{3}$ & 0 & $u_{3}$ & $\lambda u+\lambda_{5} g$ & $u_{1}$ & $u_{2}$ & $\xi u+\lambda_{6} g$ & 0 & 0 \\
\hline$g_{1}$ & $g_{1}$ & 0 & $g_{2}+\delta_{1} u_{2}$ & $g_{3}+\delta_{2} u_{3}$ & $\delta_{3} u+\lambda_{7} g$ & $\delta_{4} u_{2}$ & $\delta_{5} u_{3}$ & $\delta_{6}+\lambda_{8} g$ \\
\hline$g_{2}$ & $g_{2}$ & $g_{2}$ & $\delta_{7} u_{1}$ & $\delta_{8} u+\lambda_{9} g$ & $g_{3}+\delta_{9} u_{3}$ & $g_{1}+\delta_{10} u_{1}$ & $\delta_{11} u+\lambda_{10} g$ & $\delta_{12} u_{3}$ \\
\hline$g_{3}$ & 0 & $g_{3}$ & $\delta_{13} u+\lambda_{11} g$ & $\delta_{14} u_{1}$ & $\delta_{15} u_{2}$ & $\delta_{16} u+\lambda_{12} g$ & $g_{1}+\delta_{17} u_{1}$ & $g_{2}+\delta_{18} u_{2}$ \\
\hline$g$ & 0 & 0 & 0 & 0 & 0 & 0 & 0 & 0 \\
\hline
\end{tabular}

Tabela 3.60: $O \mathfrak{s l}_{3}(k)-2$-módulos $U^{145}:=\left[V_{3} \wedge\left(V_{3}^{*} \curlywedge V_{0}\right)\right] \curlywedge V_{0}$

Da tabela anterior temos que:

$$
U^{145} \simeq\left[V_{3} \oplus\left(V_{3}^{*} \oplus V_{0}\right)\right] \oplus V_{0} \Longleftrightarrow \delta_{i}=\lambda_{j}=\pi=\xi=\lambda=0 .
$$


43. Tabela para o $\mathfrak{s l}_{3}(k)-2$-módulo $U^{148}:=\left[\left(V_{0} \wedge V_{3}^{*}\right) \curlywedge V_{3}\right] \curlywedge V_{0}$

\begin{tabular}{|c|c|c|c|c|c|c|c|c|}
\hline & $h_{1}$ & $h_{2}$ & $e_{12}$ & $e_{13}$ & $e_{23}$ & $e_{21}$ & $e_{31}$ & $e_{32}$ \\
\hline \hline$v$ & 0 & 0 & 0 & 0 & 0 & 0 & 0 & 0 \\
\hline$v_{1}$ & $v_{1}$ & 0 & $v_{2}$ & $v_{3}$ & $\lambda_{1} v$ & 0 & 0 & $\lambda_{2} v$ \\
\hline$v_{2}$ & $v_{2}$ & $v_{2}$ & 0 & $\lambda_{3} v$ & $v_{3}$ & $v_{1}$ & $\lambda_{4} v$ & 0 \\
\hline$v_{3}$ & 0 & $v_{3}$ & $\lambda_{5} v$ & 0 & 0 & $\lambda_{6} v$ & $v_{1}$ & $v_{2}$ \\
\hline$O_{1}$ & $o_{1}$ & 0 & $\delta_{7} v_{2}$ & $\delta_{8} v_{3}$ & $\lambda_{7} v$ & $o_{2}+\delta_{9} v_{2}$ & $o_{3}+\delta_{10} v_{3}$ & $\lambda_{8} v$ \\
\hline$O_{2}$ & $o_{2}$ & $o_{2}$ & $o_{1}+\delta_{11} v_{1}$ & $\lambda_{9} v$ & $\delta_{12} v_{3}$ & $\delta_{13} v_{1}$ & $\lambda_{10} v$ & $o_{3}+\delta_{14} v_{3}$ \\
\hline$O_{3}$ & 0 & $o_{3}$ & $\lambda_{11} v$ & $o_{1}+\delta_{15} v_{1}$ & $o_{2}+\delta_{16} v_{2}$ & $\lambda_{12} v$ & $\delta_{17} v_{1}$ & $\delta_{18} v_{2}$ \\
\hline$O$ & 0 & 0 & $\delta o_{3}+\delta_{1} v_{3}$ & $\delta_{o_{2}}+\delta_{2} v_{2}$ & $\alpha o_{1}+\delta_{3} v_{1}$ & $\alpha o_{3}+\delta_{4} v_{3}$ & $\zeta o_{2}+\delta_{5} v_{2}$ & $\zeta o_{1}+\delta_{6} v_{1}$ \\
\hline
\end{tabular}

Tabela 3.61: $O \mathfrak{s l}_{3}(k)-2$-módulos $U^{148}:=\left[\left(V_{0} \curlywedge V_{3}^{*}\right) \curlywedge V_{3}\right] \curlywedge V_{0}$

Da tabela anterior temos que:

$$
U^{148} \simeq\left[\left(V_{0} \oplus V_{3}^{*}\right) \oplus V_{3}\right] \oplus V_{0} \Longleftrightarrow \delta_{i}=\lambda_{j}=\delta=\alpha=\zeta=0 .
$$

44. Tabela para o $\mathfrak{s l}_{3}(k)-2$-módulo $U^{149}:=\left[V_{3} \curlywedge\left(V_{0} \curlywedge V_{3}^{*}\right)\right] \curlywedge V_{0}$

\begin{tabular}{|c|c|c|c|c|c|c|c|c|}
\hline & $h_{1}$ & $h_{2}$ & $e_{12}$ & $e_{13}$ & $e_{23}$ & $e_{21}$ & $e_{31}$ & $e_{32}$ \\
\hline$y$ & 0 & 0 & 0 & 0 & 0 & 0 & 0 & 0 \\
\hline$w_{1}$ & $w_{1}$ & 0 & 0 & 0 & $\lambda_{1} y$ & $w_{2}$ & $w_{3}$ & $\lambda_{2} y$ \\
\hline$w_{2}$ & $w_{2}$ & $w_{2}$ & $w_{1}$ & $\lambda_{3} y$ & 0 & 0 & $\lambda_{4} y$ & $w_{3}$ \\
\hline$w_{3}$ & 0 & $w_{3}$ & $\lambda_{5} y$ & $w_{1}$ & $w_{2}$ & $\lambda_{6} y$ & 0 & 0 \\
\hline$y_{1}$ & $y_{1}$ & 0 & $y_{2}+\delta_{1} w_{2}$ & $y_{3}+\delta_{2} w_{3}$ & $\delta_{3} w+\lambda_{7} y$ & $\delta_{4} w_{2}$ & $\delta_{5} w_{3}$ & $\delta_{6} w+\lambda_{8} y$ \\
\hline$y_{2}$ & $y_{2}$ & $y_{2}$ & $\delta_{7} w_{1}$ & $\delta_{8} w+\lambda_{9} y$ & $y_{3}+\delta_{9} w_{3}$ & $y_{1}+\delta_{10} w_{1}$ & $\delta_{11} w+\lambda_{10} y$ & $\delta_{12} w_{3}$ \\
\hline$y_{3}$ & 0 & $y_{3}$ & $\delta_{13} w+\lambda_{11} y$ & $\delta_{14} w_{1}$ & $\delta_{15} w_{2}$ & $\delta_{16} w+\lambda_{12} y$ & $y_{1}+\delta_{17} w_{1}$ & $y_{2}+\delta_{18} w_{2}$ \\
\hline$w$ & 0 & 0 & $\delta w_{3}$ & $\delta w_{2}$ & $\alpha w_{1}$ & $\alpha w_{3}$ & $\zeta w_{2}$ & $\zeta w_{1}$ \\
\hline
\end{tabular}

Tabela 3.62: $O \mathfrak{s l}_{3}(k)-2$-módulos $U^{149}:=\left[V_{3} \curlywedge\left(V_{0} \curlywedge V_{3}^{*}\right)\right] \curlywedge V_{0}$

Da tabela anterior temos que:

$$
U^{149} \simeq\left[V_{3} \oplus\left(V_{0} \oplus V_{3}^{*}\right)\right] \oplus V_{0} \Longleftrightarrow \delta_{i}=\lambda_{j}=\delta=\alpha=\zeta=0 .
$$


45. Tabela para o $\mathfrak{s l}_{3}(k)-2-$ módulo $U^{178}:=\left[\left(V_{3}^{*} \wedge V_{3}\right) \wedge V_{0}\right] \wedge V_{0}$

\begin{tabular}{|c||c|c|c|c|c|c|c|c|}
\hline & $h_{1}$ & $h_{2}$ & $e_{12}$ & $e_{13}$ & $e_{23}$ & $e_{21}$ & $e_{31}$ & $e_{32}$ \\
\hline \hline$x$ & 0 & 0 & 0 & 0 & 0 & 0 & 0 & 0 \\
\hline$x_{1}$ & $x_{1}$ & 0 & $x_{2}$ & $x_{3}$ & $\delta_{1} x+\lambda_{1} y$ & 0 & 0 & $\delta_{2} x+\lambda_{2} y$ \\
\hline$x_{2}$ & $x_{2}$ & $x_{2}$ & 0 & $\delta_{3} x+\lambda_{3} y$ & $x_{3}$ & $x_{1}$ & $\delta_{4} x+\lambda_{4} y$ & 0 \\
\hline$x_{3}$ & 0 & $x_{3}$ & $\delta_{5} x+\lambda_{5} y$ & 0 & 0 & $\delta_{6} x+\lambda_{6} y$ & $x_{1}$ & $x_{2}$ \\
\hline$y_{1}$ & $y_{1}$ & 0 & $\alpha x_{2}$ & $\alpha x_{3}$ & $\delta_{7} x+\lambda_{7} y$ & $y_{2}+\gamma x_{2}$ & $y_{3}+\beta x_{3}$ & $\delta_{8} x+\lambda_{8} y$ \\
\hline$y_{2}$ & $y_{2}$ & $y_{2}$ & $y_{1}+\alpha x_{1}$ & $\delta_{9} x+\lambda_{9} y$ & $\gamma x_{3}$ & $\gamma x_{1}$ & $\delta_{10} x+\lambda_{10} y$ & $y_{3}+\beta x_{3}$ \\
\hline$y_{3}$ & 0 & $y_{3}$ & $\delta_{11} x+\lambda_{11} y$ & $y_{1}+\alpha y_{1}$ & $y_{2}+\gamma x_{2}$ & $\delta_{12} x+\lambda_{12} y$ & $\beta x_{1}$ & $\beta x_{2}$ \\
\hline$y$ & 0 & 0 & 0 & 0 & 0 & 0 & 0 & 0 \\
\hline
\end{tabular}

Tabela 3.63: $O \mathfrak{s l}_{3}(k)-2$-módulos $U^{178}:=\left[\left(V_{3}^{*} \curlywedge V_{3}\right) \curlywedge V_{0}\right] \curlywedge V_{0}$

Da tabela anterior temos que:

$$
U^{178} \simeq\left[\left(V_{3}^{*} \oplus V_{3}\right) \oplus V_{0}\right] \oplus V_{0} \Longleftrightarrow \delta_{i}=\lambda_{j}=\alpha=\beta=\gamma=0 .
$$

46. Tabela para o $\mathfrak{s l}_{3}(k)-2$-módulo $U^{179}:=\left[V_{0} \curlywedge\left(V_{3} \curlywedge V_{3}^{*}\right)\right] \curlywedge V_{0}$

\begin{tabular}{|c||c|c|c|c|c|c|c|c|}
\hline & $h_{1}$ & $h_{2}$ & $e_{12}$ & $e_{13}$ & $e_{23}$ & $e_{21}$ & $e_{31}$ & $e_{32}$ \\
\hline \hline$x$ & 0 & 0 & $\delta_{1} x_{3}+\delta_{2} y_{3}$ & $\delta_{3} x_{2}+\delta_{4} y_{2}$ & $\delta_{5} x_{1}+\delta_{6} y_{1}$ & $\delta_{7} x_{3}+\delta_{8} y_{3}$ & $\delta_{9} x_{2}+\delta_{10} y_{2}$ & $\delta_{11} x_{1}+\delta_{12} y_{1}$ \\
\hline$x_{1}$ & $x_{1}$ & 0 & 0 & 0 & $\lambda_{1} y$ & $x_{2}$ & $x_{3}$ & $\lambda_{2} y$ \\
\hline$x_{2}$ & $x_{2}$ & $x_{2}$ & $x_{1}$ & $\lambda_{3} y$ & 0 & 0 & $\lambda_{4} y$ & $x_{3}$ \\
\hline$x_{3}$ & 0 & $x_{3}$ & $\lambda_{5} y$ & $x_{1}$ & $x_{2}$ & $\lambda_{6} y$ & 0 & 0 \\
\hline$y_{1}$ & $y_{1}$ & 0 & $y_{2}+\alpha x_{2}$ & $y_{3}+\gamma x_{3}$ & $\lambda_{7} y$ & $\beta x_{2}$ & $\beta x_{3}$ & $\lambda_{8} y$ \\
\hline$y_{2}$ & $y_{2}$ & $y_{2}$ & $\alpha x_{1}$ & $\lambda_{9} y$ & $y_{3}+\gamma x_{3}$ & $y_{1}+\beta x_{1}$ & $\lambda_{10} y$ & 0 \\
\hline$y_{3}$ & 0 & $y_{3}$ & $\lambda_{11} y$ & $\gamma x_{1}$ & $\gamma x_{2}$ & $\lambda_{12} y$ & $y_{1}+\beta x_{1}$ & $x_{2}$ \\
\hline$y$ & 0 & 0 & 0 & 0 & 0 & 0 & 0 & 0 \\
\hline
\end{tabular}

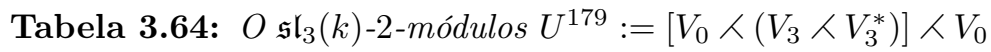

Da tabela anterior temos que:

$$
U^{179} \simeq\left[V_{0} \oplus\left(V_{3} \oplus V_{3}^{*}\right)\right] \oplus V_{0} \Longleftrightarrow \delta_{i}=\lambda_{j}=\alpha=\beta=\gamma=0 .
$$


47. Tabela para o $\mathfrak{s l}_{3}(k)-2$-módulo $U^{180}:=\left[\left(V_{3} \wedge V_{3}^{*}\right) \curlywedge V_{0}\right] \curlywedge V_{0}$

\begin{tabular}{|c||c|c|c|c|c|c|c|c|}
\hline & $h_{1}$ & $h_{2}$ & $e_{12}$ & $e_{13}$ & $e_{23}$ & $e_{21}$ & $e_{31}$ & $e_{32}$ \\
\hline \hline$k$ & 0 & 0 & 0 & 0 & 0 & 0 & 0 & 0 \\
\hline$k_{1}$ & $k_{1}$ & 0 & 0 & 0 & $\delta_{1} k+\lambda_{1} n$ & $k_{2}$ & $k_{3}$ & $\delta_{2} k+\lambda_{2} n$ \\
\hline$k_{2}$ & $k_{2}$ & $k_{2}$ & $k_{1}$ & $\delta_{3} k+\lambda_{3} n$ & 0 & 0 & $\delta_{4} k+\lambda_{4} n$ & $k_{3}$ \\
\hline$k_{3}$ & 0 & $k_{3}$ & $\delta_{5} k+\lambda_{5} n$ & $k_{1}$ & $k_{2}$ & $\delta_{6} k+\lambda_{6} n$ & 0 & 0 \\
\hline$n_{1}$ & $n_{1}$ & 0 & $n_{2}+\alpha k_{2}$ & $n_{3}+\gamma k_{3}$ & $\delta_{7} k+\lambda_{7} n$ & $\beta k_{2}$ & $\beta k_{3}$ & $\delta_{8} k+\lambda_{8} n$ \\
\hline$n_{2}$ & $n_{2}$ & $n_{2}$ & $\alpha k_{1}$ & $\delta_{9} k+\lambda_{9} n$ & $n_{3}+\gamma k_{3}$ & $n_{1}+\beta k_{1}$ & $\delta_{10} k+\lambda_{10} n$ & $\alpha k_{3}$ \\
\hline$n_{3}$ & 0 & $n_{3}$ & $\delta_{11} k+\lambda_{11} n$ & $\gamma k_{1}$ & $\gamma k_{2}$ & $\delta_{12} k+\lambda_{12} n$ & $n_{1}+\beta k_{1}$ & $n_{2}+\alpha k_{2}$ \\
\hline$n$ & 0 & 0 & 0 & 0 & 0 & 0 & 0 & 0 \\
\hline
\end{tabular}

Tabela 3.65: $O \mathfrak{s l}_{3}(k)-2-$ módulos $U^{180}:=\left[\left(V_{3} \wedge V_{3}^{*}\right) \curlywedge V_{0}\right] \curlywedge V_{0}$

Da tabela anterior temos que:

$$
U^{180} \simeq\left[\left(V_{3} \oplus V_{3}^{*}\right) \oplus V_{0}\right] \oplus V_{0} \Longleftrightarrow \delta_{i}=\lambda_{j}=\alpha=\beta=\gamma=0 .
$$

48. Tabela para o $\mathfrak{s l}_{3}(k)-2$-módulo $U^{184}:=\left[V_{0} \curlywedge\left(V_{3}^{*} \wedge V_{3}\right)\right] \wedge V_{0}$

\begin{tabular}{|c||c|c|c|c|c|c|c|c|}
\hline & $h_{1}$ & $h_{2}$ & $e_{12}$ & $e_{13}$ & $e_{23}$ & $e_{21}$ & $e_{31}$ & $e_{32}$ \\
\hline \hline$x$ & 0 & 0 & $\delta_{1} x_{3}+\delta_{2} y_{3}$ & $\delta_{3} x_{2}+\delta_{4} y_{2}$ & $\delta_{5} x_{1}+\delta_{6} y_{1}$ & $\delta_{7} x_{3}+\delta_{8} y_{3}$ & $\delta_{9} x_{2}+\delta_{10} y_{2}$ & $\delta_{11} x_{1}+\delta_{12} y_{1}$ \\
\hline$x_{1}$ & $x_{1}$ & 0 & $x_{2}$ & $x_{3}$ & $\delta_{13} y$ & 0 & 0 & $\delta_{14} y$ \\
\hline$x_{2}$ & $x_{2}$ & $x_{2}$ & 0 & $\delta_{15} y$ & $x_{3}$ & $x_{1}$ & $\delta_{16} y$ & 0 \\
\hline$x_{3}$ & 0 & $x_{3}$ & $\delta_{17} y$ & 0 & 0 & $\delta_{18} y$ & $x_{1}$ & $x_{2}$ \\
\hline$y_{1}$ & $y_{1}$ & 0 & $\alpha x_{2}$ & $\gamma x_{3}$ & $\delta_{19} y$ & $y_{2}+\gamma x_{2}$ & $y_{3}+\beta x_{3}$ & $\delta_{20} y$ \\
\hline$y_{2}$ & $y_{2}$ & $y_{2}$ & $y_{1}+\alpha x_{1}$ & $\delta_{21} y$ & $\gamma x_{3}$ & $\gamma x_{1}$ & $\delta_{22} y$ & $y_{3}+\beta x_{3}$ \\
\hline$y_{3}$ & 0 & $y_{3}$ & $\delta_{23} y$ & $y_{1}+\alpha x_{1}$ & $y_{2}+\gamma x_{2}$ & $\delta_{24} y$ & $\beta x_{1}$ & $\beta x_{2}$ \\
\hline$y$ & 0 & 0 & 0 & 0 & 0 & 0 & 0 & 0 \\
\hline
\end{tabular}

Tabela 3.66: $O \mathfrak{s l}_{3}(k)-2$-módulos $U^{184}:=\left[V_{0} \curlywedge\left(V_{3}^{*} \curlywedge V_{3}\right)\right] \curlywedge V_{0}$

Da tabela anterior temos que:

$$
U^{184} \simeq\left[V_{0} \oplus\left(V_{3}^{*} \oplus V_{3}\right)\right] \oplus V_{0} \Longleftrightarrow \delta_{i}=\alpha=\beta=\gamma=0 .
$$


49. Tabela para o $\mathfrak{s l}_{3}(k)-2-$ módulo $U^{253}:=V_{0} \curlywedge\left[\left(V_{3} \wedge V_{0}\right) \curlywedge V_{3}^{*}\right]$

\begin{tabular}{|c||c|c|c|c|c|c|c|c|}
\hline & $h_{1}$ & $h_{2}$ & $e_{12}$ & $e_{13}$ & $e_{23}$ & $e_{21}$ & $e_{31}$ & $e_{32}$ \\
\hline \hline$l_{1}$ & $l_{1}$ & 0 & 0 & 0 & 0 & $l_{2}$ & 0 & $l_{3}$ \\
\hline$l_{2}$ & $l_{2}$ & $l_{2}$ & $l_{1}$ & 0 & 0 & 0 & 0 & 0 \\
\hline$l_{3}$ & 0 & $l_{3}$ & 0 & $l_{1}$ & $l_{2}$ & 0 & $l_{3}$ \\
\hline$u_{1}$ & $u_{1}$ & 0 & $u_{2}+\delta_{7} l_{2}$ & $u_{3}+\delta_{8} l_{3}$ & $\beta u$ & $\delta_{9} l_{2}$ & $\delta_{10} l_{3}$ & \multicolumn{1}{c|}{${ }$} \\
\hline$u_{2}$ & $u_{2}$ & $u_{2}$ & $\delta_{11} l_{1}$ & $\gamma u$ & $u_{3}+\delta_{12} l_{3}$ & $u_{1}+\delta_{13} l_{1}$ & $\lambda u$ & $\delta_{14} l_{3}$ \\
\hline$u_{3}$ & 0 & $u_{3}$ & $\gamma u$ & $\delta_{15} l_{1}$ & $\delta_{16} l_{2}$ & $\beta u$ & $u_{1}+\delta_{17} l_{1}$ & $u_{2}+\delta_{18} l_{2}$ \\
\hline$b$ & 0 & 0 & $\delta_{18} l_{3}+\delta_{19} u_{3}$ & $\delta_{20} l_{2}+\delta_{21} u_{2}$ & $\delta_{22} l_{1}+\delta_{23} u_{1}$ & $\delta_{24} l_{3}+\delta_{25} u_{3}$ & $\delta_{26} l_{2}+\delta_{27} u_{2}$ & $\delta_{28} l_{1}+\delta_{29} u_{1}$ \\
\hline$u$ & 0 & 0 & $\delta_{1} l_{3}$ & $\delta_{2} l_{2}$ & $\delta_{3} l_{1}$ & $\delta_{4} l_{3}$ & $\delta_{5} l_{2}$ & $\delta_{6} l_{1}$ \\
\hline
\end{tabular}

Tabela 3.67: $O \mathfrak{s l}_{3}(k)-2-$ módulos $U^{253}:=V_{0} \curlywedge\left[\left(V_{3} \wedge V_{0}\right) \curlywedge V_{3}^{*}\right]$

Da tabela anterior temos que:

$$
U^{253} \simeq V_{0} \oplus\left[\left(V_{3} \wedge V_{0}\right) \oplus V_{3}^{*}\right] \Longleftrightarrow \delta_{i}=0 .
$$

50. Tabela para o $\mathfrak{s l}_{3}(k)-2-$ módulo $U^{254}:=V_{0} \curlywedge\left[V_{3}^{*} \curlywedge\left(V_{3} \curlywedge V_{0}\right)\right]$

\begin{tabular}{|c||c|c|c|c|c|c|c|c|}
\hline & $h_{1}$ & $h_{2}$ & $e_{12}$ & $e_{13}$ & $e_{23}$ & $e_{21}$ & $e_{31}$ & $e_{32}$ \\
\hline \hline$a$ & 0 & 0 & 0 & 0 & 0 & 0 & 0 & 0 \\
\hline$a_{1}$ & $a_{1}$ & 0 & $a_{2}$ & $a_{3}$ & $\beta a$ & 0 & $\kappa$ & \\
\hline$a_{2}$ & $a_{2}$ & $a_{2}$ & 0 & $\gamma a$ & $a_{3}$ & $a_{1}$ & $a_{1}$ & 0 \\
\hline$a_{3}$ & 0 & $a_{3}$ & $\gamma a$ & 0 & 0 & $\beta a$ & $a_{2}$ \\
\hline$c_{1}$ & $c_{1}$ & 0 & $\delta_{1} a_{2}$ & $\delta_{2} a_{3}$ & $\delta_{3} a$ & $c_{2}+\delta_{4} a_{2}$ & $c_{3}+\delta_{5} a_{3}$ & $\delta_{6} a$ \\
\hline$c_{2}$ & $c_{2}$ & $c_{2}$ & $c_{1}+\delta_{7} a_{1}$ & $\delta_{8} a$ & $\delta_{9} a_{3}$ & $\delta_{10} a_{1}$ & $\delta_{11} a$ & $c_{3}+\delta_{12} a_{3}$ \\
\hline$c_{3}$ & 0 & $c_{3}$ & $\delta_{13} a$ & $c_{1}+\delta_{14} a_{1}$ & $c_{2}+\delta_{15} a_{2}$ & $\delta_{16} a$ & $\delta_{17} a_{1}$ & $\delta_{18} a_{2}$ \\
\hline$u$ & 0 & 0 & $\delta_{19} a_{3}+\delta_{20} c_{3}$ & $\delta_{21} a_{2}+\delta_{22} c_{2}$ & $\delta_{23} a_{1}+\delta_{24} c_{1}$ & $\delta_{25} a_{3}+\delta_{26} c_{3}$ & $\delta_{27} a_{2}+\delta_{28} c_{2}$ & $\delta_{29} a_{1}+\delta_{30} c_{1}$ \\
\hline
\end{tabular}

Tabela 3.68: $O \mathfrak{s l}_{3}(k)-2$-módulos $U^{254}:=V_{0} \curlywedge\left[V_{3}^{*} \curlywedge\left(V_{3} \wedge V_{0}\right)\right]$

Da tabela anterior temos que:

$$
U^{254} \simeq V_{0} \oplus\left[\left(V_{3}^{*} \oplus\left(V_{3} \curlywedge V_{0}\right)\right] \Longleftrightarrow \delta_{i}=0 .\right.
$$


51. Tabela para o $\mathfrak{s l}_{3}(k)-2$-módulo $U^{257}:=V_{0} \curlywedge\left[\left(V_{0} \curlywedge V_{3}\right) \curlywedge V_{3}^{*}\right]$

\begin{tabular}{|c||c|c|c|c|c|c|c|c|}
\hline & $h_{1}$ & $h_{2}$ & $e_{12}$ & $e_{13}$ & $e_{23}$ & $e_{21}$ & $e_{31}$ & $e_{32}$ \\
\hline \hline$l_{1}$ & 0 & 0 & 0 & 0 & 0 & $l_{2}$ & $l_{3}$ & 0 \\
\hline$l_{2}$ & $l_{2}$ & $l_{2}$ & $l_{1}$ & 0 & 0 & 0 & 0 & $l_{3}$ \\
\hline$l_{3}$ & 0 & $l_{3}$ & 0 & $l_{1}$ & $l_{2}$ & 0 & 0 \\
\hline$i$ & 0 & 0 & $\omega i_{3}+\delta_{1} l_{3}$ & $\theta i_{2}+\delta_{2} l_{2}$ & $\theta i_{1}+\delta_{3} l_{1}$ & $\sigma i_{3}+\delta_{4} l_{3}$ & $\sigma i_{2}+\delta_{5} l_{2}$ & $\omega i_{1}+\delta_{6} l_{1}$ \\
\hline$i_{1}$ & $i_{1}$ & 0 & $i_{2}+\delta_{7} l_{2}$ & $i_{3}+\delta_{8} l_{3}$ & 0 & $\delta_{10} l_{2}$ & $\delta_{11} l_{3}$ & 0 \\
\hline$i_{2}$ & $i_{2}$ & $i_{2}$ & $\delta_{7} l_{2}$ & 0 & $i_{3}+\delta_{9} l_{3}$ & $i_{1}+\delta_{10} l_{1}$ & 0 & $\delta_{12} l_{3}$ \\
\hline$i_{3}$ & 0 & $i_{3}$ & 0 & $\delta_{8} l_{1}$ & $\delta_{9} l_{2}$ & 0 & $i_{1}+\delta_{11} l_{1}$ & $a_{2}+\delta_{12} l_{2}$ \\
\hline$u$ & 0 & 0 & $\delta_{13} i_{3}+\delta_{14} l_{3}$ & $\delta_{15} i_{2}+\delta_{16} l_{2}$ & $\delta_{17} i_{1}+\delta_{18} l_{1}$ & $\delta_{19} i_{3}+\delta_{20} l_{3}$ & $\delta_{21} i_{2}+\delta_{22} l_{2}$ & $\delta_{23} i_{1}+\delta_{24} l_{1}$ \\
\hline
\end{tabular}

Tabela 3.69: $O \mathfrak{s l}_{3}(k)-2$-módulos $\left.U^{257}:=V_{0} \wedge\left[\left(V_{0} \wedge V_{3}\right) \wedge V_{3}^{*}\right)\right]$

Da tabela anterior temos que:

$$
\left.U^{257} \simeq V_{0} \oplus\left[\left(V_{0} \oplus V_{3}\right) \oplus V_{3}^{*}\right)\right] \Longleftrightarrow \delta_{i}=\omega=\theta=\sigma=0
$$

52. Tabela para o $\mathfrak{s l}_{3}(k)-2$-módulo $U^{258}:=V_{0} \curlywedge\left[V_{3}^{*} \curlywedge\left(V_{0} \wedge V_{3}\right)\right]$

\begin{tabular}{|c||c|c|c|c|c|c|c|c|}
\hline & $h_{1}$ & $h_{2}$ & $e_{12}$ & $e_{13}$ & $e_{23}$ & $e_{21}$ & $e_{31}$ & $e_{32}$ \\
\hline \hline$v$ & 0 & 0 & $\omega v_{3}$ & $\theta v_{2}$ & $\theta v_{1}$ & $\sigma v_{3}$ & $\sigma v_{2}$ & $\omega v_{1}$ \\
\hline$v_{1}$ & $v_{1}$ & 0 & $v_{2}$ & $v_{3}$ & 0 & 0 & 0 & 0 \\
\hline$v_{2}$ & $v_{2}$ & $v_{2}$ & 0 & 0 & $v_{3}$ & $v_{1}$ & $v_{1}$ & 0 \\
\hline$v_{3}$ & 0 & $v_{3}$ & 0 & 0 & 0 & 0 & $v_{2}$ \\
\hline$i_{1}$ & $i_{1}$ & 0 & $\delta_{1} v_{2}$ & $\delta_{2} v_{3}$ & $\delta_{3} v$ & $i_{2}+\delta_{4} v_{2}$ & $i_{3}+\delta_{5} v_{3}$ & $\delta_{6} v$ \\
\hline$i_{2}$ & $i_{2}$ & $i_{2}$ & $i_{1}+\delta_{7} v_{2}$ & $\delta_{6} v$ & $\delta_{8} v_{3}$ & $\delta_{9} v_{1}$ & $\delta_{10} v$ & $i_{3}+\delta_{11} v_{3}$ \\
\hline$i_{3}$ & 0 & $i_{3}$ & $\delta_{12} v$ & $i_{1}+\delta_{13} v_{1}$ & $i_{2}+\delta_{14} v_{2}$ & $\delta_{15} v$ & $\delta_{16} v_{1}$ & $\delta_{17} v_{2}$ \\
\hline$u$ & 0 & 0 & $\delta_{18} i_{3}+\delta_{19} v_{3}$ & $\delta_{20} i_{2}+\delta_{21} v_{2}$ & $\delta_{22} i_{1}+\delta_{23} v_{1}$ & $\delta_{24} i_{3}+\delta_{25} v_{3}$ & $\delta_{26} i_{2}+\delta_{27} v_{2}$ & $\delta_{28} i_{1}+\delta_{29} v_{1}$ \\
\hline
\end{tabular}

Tabela 3.70: $O \mathfrak{s l}_{3}(k)$-2-módulos $U^{258}:=V_{0} \curlywedge\left[V_{3}^{*} \curlywedge\left(V_{0} \wedge V_{3}\right)\right]$

Da tabela anterior temos que:

$$
U^{258} \simeq V_{0} \oplus\left[V_{3}^{*} \oplus\left(V_{0} \oplus V_{3}\right)\right] \Longleftrightarrow \delta_{i}=\omega=\theta=\sigma=0
$$


53. Tabela para o $\mathfrak{s l}_{3}(k)-2-$ módulo $U^{259}:=V_{0} \curlywedge\left[\left(V_{3}^{*} \curlywedge V_{0}\right) \curlywedge V_{3}\right]$

\begin{tabular}{|c||c|c|c|c|c|c|c|c|}
\hline & $h_{1}$ & $h_{2}$ & $e_{12}$ & $e_{13}$ & $e_{23}$ & $e_{21}$ & $e_{31}$ & $e_{32}$ \\
\hline \hline$i$ & 0 & 0 & $\delta_{1} v_{3}$ & $\delta_{2} v_{2}$ & $\delta_{3} v_{1}$ & $\delta_{4} v_{3}$ & $\delta_{5} v_{2}$ & $\delta_{6} v_{1}$ \\
\hline$v_{1}$ & $v_{1}$ & 0 & $v_{2}$ & $v_{3}$ & 0 & 0 & 0 & 0 \\
\hline$v_{2}$ & $v_{2}$ & $v_{2}$ & 0 & 0 & $v_{3}$ & $v_{1}$ & 0 & 0 \\
\hline$v_{3}$ & 0 & $v_{3}$ & 0 & 0 & 0 & 0 & $v_{1}$ & $v_{2}$ \\
\hline$i_{1}$ & $i_{1}$ & 0 & $\delta_{7} v_{2}$ & $\delta_{8} v_{3}$ & $\pi i$ & $i_{2}+\delta_{9} v_{2}$ & $i_{3}+\delta_{10} v_{3}$ & $i_{3}+\delta_{14} v_{3}$ \\
\hline$i_{2}$ & $i_{2}$ & $i_{2}$ & $i_{1}+\delta_{11} v_{2}$ & $\pi i$ & $\delta_{12} v_{3}$ & $\delta_{13} v_{1}$ & $\xi i$ & $\delta_{18} v_{1}$ \\
\hline$i_{3}$ & 0 & $i_{3}$ & $\lambda i$ & $i_{1}+\delta_{15} v_{1}$ & $i_{2}+\delta_{16} v_{2}$ & $\xi i$ & $\delta_{12}$ & $\delta_{29} v_{1}$ \\
\hline$u$ & 0 & 0 & $\delta_{19} i_{3}+\delta_{20} v_{3}$ & $\delta_{21} i_{2}+\delta_{22} v_{2}$ & $\delta_{23} i_{1}+\delta_{24} v_{1}$ & $\delta_{25} i_{3}+\delta_{26} v_{3}$ & $\delta_{27} i_{2}+\delta_{28} v_{2}$ & $\delta_{29} i_{1}+\delta_{30} v_{1}$ \\
\hline
\end{tabular}

Tabela 3.71: $O \mathfrak{s l}_{3}(k)-2-$ módulos $U^{259}:=V_{0} \curlywedge\left[\left(V_{3}^{*} \wedge V_{0}\right) \curlywedge V_{3}\right]$

Da tabela anterior temos que:

$$
U^{259} \simeq V_{0} \oplus\left[\left(V_{3}^{*} \wedge V_{0}\right) \oplus V_{3}\right] \Longleftrightarrow \delta_{i}=0 .
$$

54. Tabela para o $\mathfrak{s l}_{3}(k)-2$-módulo $U^{260}:=V_{0} \curlywedge\left[V_{3} \curlywedge\left(V_{3}^{*} \curlywedge V_{0}\right)\right]$

\begin{tabular}{|c||c|c|c|c|c|c|c|c|}
\hline & $h_{1}$ & $h_{2}$ & $e_{12}$ & $e_{13}$ & $e_{23}$ & $e_{21}$ & $e_{31}$ & $e_{32}$ \\
\hline \hline$u$ & 0 & 0 & 0 & 0 & 0 & 0 & 0 \\
\hline$u_{1}$ & $u_{1}$ & 0 & 0 & 0 & $\pi u$ & $u_{2}$ & $u_{3}$ & \\
\hline$u_{2}$ & $u_{2}$ & $u_{2}$ & $u_{1}$ & $\pi u$ & 0 & 0 & $\xi u$ \\
\hline$u_{3}$ & 0 & $u_{3}$ & $\lambda u$ & $u_{1}$ & $u_{2}$ & $\xi u$ & $u_{3}$ \\
\hline$g_{1}$ & $g_{1}$ & 0 & $\delta_{1} u_{2}$ & $\delta_{2} u_{3}$ & $\delta_{3} u$ & $g_{2}+\delta_{4} u_{2}$ & $g_{3}+\delta_{5} u_{3}$ & $\delta_{6} u$ \\
\hline$g_{2}$ & $g_{2}$ & $g_{2}$ & $g_{1}+\delta_{7} u_{1}$ & $\delta_{8} u$ & $\delta_{9} u_{3}$ & $\delta_{10} u_{1}$ & $\delta_{11} u$ & $g_{3}+\delta_{12} u_{3}$ \\
\hline$g_{3}$ & 0 & $g_{3}$ & $\delta_{13} u$ & $g_{1}+\delta_{14} u_{1}$ & $g_{2}+\delta_{15} u_{2}$ & $\delta_{16} u$ & $\delta_{17} u_{1}$ & $\delta_{18} u_{2}$ \\
\hline$g$ & 0 & 0 & $\delta_{19} g_{3}+\delta_{20} u_{3}$ & $\delta_{21} g_{2}+\delta_{22} u_{2}$ & $\delta_{23} g_{1}+\delta_{24} u_{1}$ & $\delta_{25} g_{3}+\delta_{26} u_{3}$ & $\delta_{27} g_{2}+\delta_{28} u_{2}$ & $\delta_{29} g_{1}+\delta_{30} u_{1}$ \\
\hline
\end{tabular}

Tabela 3.72: $O \mathfrak{s l}_{3}(k)-2$-módulos $U^{260}:=V_{0} \curlywedge\left[V_{3} \curlywedge\left(V_{3}^{*} \curlywedge V_{0}\right)\right]$

Da tabela anterior temos que:

$$
U^{260} \simeq V_{0} \oplus\left[V_{3} \oplus\left(V_{3}^{*} \wedge V_{0}\right)\right] \Longleftrightarrow \delta_{i}=0 .
$$


55. Tabela para o $\mathfrak{s l}_{3}(k)-2$-módulo $U^{263}:=V_{0} \curlywedge\left[\left(V_{0} \wedge V_{3}^{*}\right) \curlywedge V_{3}\right]$

\begin{tabular}{|c|c|c|c|c|c|c|c|c|}
\hline & $h_{1}$ & $h_{2}$ & $e_{12}$ & $e_{13}$ & $e_{23}$ & $e_{21}$ & $e_{31}$ & $e_{32}$ \\
\hline \hline$v_{1}$ & 0 & 0 & $v_{2}$ & $v_{3}$ & 0 & 0 & 0 & 0 \\
\hline$v_{2}$ & $v_{2}$ & $v_{2}$ & 0 & 0 & $v_{3}$ & $v_{1}$ & 0 & $v_{1}$ \\
\hline$v_{3}$ & 0 & $v_{3}$ & 0 & 0 & 0 & 0 & $v_{2}$ \\
\hline$g$ & 0 & 0 & $\delta g_{3}+\delta_{1} v_{3}$ & $\delta g_{2}+\delta_{2} v_{2}$ & $\alpha g_{1}+\delta_{3} v_{1}$ & $\alpha g_{3}+\delta_{4} v_{3}$ & $\zeta g_{2}+\delta_{5} v_{2}$ & $\zeta g_{1}+\delta_{6} v_{1}$ \\
\hline$g_{1}$ & $g_{1}$ & 0 & $\delta_{7} v_{2}$ & $\delta_{8} v_{3}$ & 0 & $g_{2}+\delta_{9} v_{2}$ & $g_{3}+\delta_{10} v_{3}$ & 0 \\
\hline$g_{2}$ & $g_{2}$ & $g_{2}$ & $g_{1}+\delta_{11} v_{1}$ & 0 & $\delta_{12} v_{3}$ & $\delta_{13} v_{1}$ & 0 & $g_{3}+\delta_{14} v_{3}$ \\
\hline$g_{3}$ & 0 & $g_{3}$ & 0 & $g_{1}+\delta_{15} v_{1}$ & $g_{2}+\delta_{16} v_{2}$ & 0 & $\delta_{17} v_{1}$ & $\delta_{18} v_{2}$ \\
\hline$v$ & 0 & 0 & $\delta_{19} g_{3}+\delta_{20} v_{3}$ & $\delta_{21} g_{2}+\delta_{22} v_{2}$ & $\delta_{23} g_{1}+\delta_{24} v_{1}$ & $\delta_{25} g_{3}+\delta_{26} v_{3}$ & $\delta_{27} g_{2}+\delta_{28} v_{2}$ & $\delta_{29} g_{1}+\delta_{30} v_{1}$ \\
\hline
\end{tabular}

Tabela 3.73: $O \mathfrak{s l}_{3}(k)$-2-módulos $U^{263}:=V_{0} \curlywedge\left[\left(V_{0} \curlywedge V_{3}^{*}\right) \curlywedge V_{3}\right]$

Da tabela anterior temos que:

$$
U^{263} \simeq V_{0} \oplus\left[\left(V_{0} \oplus V_{3}^{*}\right) \oplus V_{3}\right] \Longleftrightarrow \delta_{i}=\alpha=\delta=\zeta=0 .
$$

56. Tabela para o $\mathfrak{s l}_{3}(k)-2-$ módulo $U^{264}:=V_{0} \curlywedge\left[V_{3} \curlywedge\left(V_{0} \curlywedge V_{3}^{*}\right)\right]$

\begin{tabular}{|c||c|c|c|c|c|c|c|c|}
\hline & $h_{1}$ & $h_{2}$ & $e_{12}$ & $e_{13}$ & $e_{23}$ & $e_{21}$ & $e_{31}$ & $e_{32}$ \\
\hline \hline$l$ & 0 & 0 & $\delta l_{3}$ & $\delta l_{2}$ & $\alpha l_{1}$ & $\alpha l_{3}$ & $\zeta l_{2}$ & $l_{3}$ \\
\hline$l_{1}$ & $l_{1}$ & 0 & 0 & 0 & 0 & $l_{2}$ & 0 & 0 \\
\hline$l_{2}$ & $l_{2}$ & $l_{1}$ & 0 & 0 & 0 & 0 & 0 & 0 \\
\hline$l_{3}$ & 0 & $l_{3}$ & 0 & $l_{1}$ & $l_{2}$ & 0 & $l_{3}$ \\
\hline$g_{1}$ & $g_{1}$ & 0 & $g_{2}+\delta_{1} l_{2}$ & $g_{3}+\delta_{2} l_{3}$ & 0 & $\delta_{3} l_{2}$ & 0 \\
\hline$g_{2}$ & $g_{2}$ & $g_{2}$ & $g_{1}+\delta_{5} l_{1}$ & 0 & $\delta_{6} l_{3}$ & $\delta_{7} l_{1}$ & $g_{3}$ & 0 \\
\hline$g_{3}$ & 0 & $g_{3}$ & 0 & $g_{1}+\delta_{9} l_{1}$ & $g_{2}+\delta_{10} l_{2}$ & 0 & $\delta_{11} l_{1}$ & $\delta_{12} l_{2}$ \\
\hline$g$ & 0 & 0 & $\delta_{13} g_{3}+\delta_{14} l_{3}$ & $\delta_{15} g_{2}+\delta_{16} l_{2}$ & $\delta_{17} g_{1}+\delta_{18} l_{1}$ & $\delta_{19} g_{3}+\delta_{20} l_{3}$ & $\delta_{21} g_{2}+\delta_{22} l_{2}$ & $\delta_{23} g_{1}+\delta_{24} l_{1}$ \\
\hline
\end{tabular}

Tabela 3.74: $O \mathfrak{s l}_{3}(k)$-2-módulos $U^{263}:=V_{0} \curlywedge\left[V_{3} \curlywedge\left(V_{0} \curlywedge V_{3}^{*}\right)\right]$

Da tabela anterior temos que:

$$
U^{263} \simeq V_{0} \oplus\left[V_{3} \oplus\left(V_{0} \oplus V_{3}^{*}\right) \Longleftrightarrow \delta_{i}=\alpha=\delta=\zeta=0 .\right.
$$


57. Tabela para o $\mathfrak{s l}_{3}(k)-2-$ módulo $U^{292}:=V_{0} \curlywedge\left[\left(V_{3}^{*} \curlywedge V_{3}\right) \curlywedge V_{0}\right]$

\begin{tabular}{|c||c|c|c|c|c|c|c|c|}
\hline & $h_{1}$ & $h_{2}$ & $e_{12}$ & $e_{13}$ & $e_{23}$ & $e_{21}$ & $e_{31}$ & $e_{32}$ \\
\hline \hline$a$ & 0 & 0 & 0 & 0 & 0 & 0 & 0 \\
\hline$a_{1}$ & $a_{1}$ & 0 & $a_{2}$ & $a_{3}$ & $\delta_{1} a$ & 0 & 0 \\
\hline$a_{2}$ & $a_{2}$ & $a_{2}$ & 0 & $\delta_{3} a$ & $a_{3}$ & $a_{1}$ & $\delta_{4} a$ & 0 \\
\hline$a_{3}$ & 0 & $a_{3}$ & $\delta_{5} a$ & 0 & 0 & $\delta_{6} a$ & $a_{1}$ & $u_{2}$ \\
\hline$u_{1}$ & $u_{1}$ & 0 & $\alpha a_{2}$ & $\alpha a_{3}$ & $\delta_{7} a$ & $u_{2}+\gamma a_{2}$ & $u_{3}+\beta a_{3}$ & $\delta_{10} a$ \\
\hline$u_{2}$ & $u_{2}$ & $u_{2}$ & $u_{1}+\alpha a_{1}$ & $\delta_{9} a$ & $\gamma a_{3}$ & $\gamma a_{1}$ & $u_{3}+\beta a_{3}$ \\
\hline$u_{3}$ & 0 & $u_{3}$ & $\delta_{11} a$ & $u_{1}+\alpha a_{1}$ & $u_{2}+\gamma a_{2}$ & $\delta_{12} a$ & $\beta a_{1}$ & $\delta_{21} a_{2}+\delta_{22} u_{2}$ \\
\hline$u$ & 0 & 0 & $\delta_{13} a_{3}+\delta_{14} u_{3}$ & $\delta_{15} a_{2}+\delta_{16} u_{2}$ & $\delta_{17} a_{1}+\delta_{18} u_{1}$ & $\delta_{19} a_{3}+\delta_{20} u_{3}$ & $\delta_{24} u_{1}$ \\
\hline
\end{tabular}

Tabela 3.75: $O \mathfrak{s l}_{3}(k)-2$-módulos $U^{292}:=V_{0} \curlywedge\left[\left(V_{3}^{*} \curlywedge V_{3}\right) \curlywedge V_{0}\right]$

Da tabela anterior temos que:

$$
U^{292} \simeq V_{0} \oplus\left[\left(V_{3}^{*} \oplus V_{3}\right) \oplus V_{0}\right] \Longleftrightarrow \delta_{i}=\alpha=\beta=\gamma=0 .
$$

58. Tabela para o $\mathfrak{s l}_{3}(k)-2-$ módulo $U^{293}:=V_{0} \curlywedge\left[V_{0} \curlywedge\left(V_{3} \curlywedge V_{3}^{*}\right)\right]$

\begin{tabular}{|c||c|c|c|c|c|c|c|c|}
\hline & $h_{1}$ & $h_{2}$ & $e_{12}$ & $e_{13}$ & $e_{23}$ & $e_{21}$ & $e_{31}$ & $e_{32}$ \\
\hline \hline$w_{1}$ & $w_{1}$ & 0 & 0 & 0 & 0 & $w_{2}$ & 0 \\
\hline$w_{2}$ & $w_{2}$ & $w_{2}$ & $w_{1}$ & 0 & 0 & 0 & 0 \\
\hline$w_{3}$ & 0 & $w_{3}$ & 0 & $w_{1}$ & $w_{2}$ & 0 & 0 \\
\hline$u_{1}$ & $u_{1}$ & 0 & $u_{2}+\alpha w_{2}$ & $u_{3}+\gamma w_{3}$ & 0 & $\beta w_{2}$ & $\beta w_{3}$ & 0 \\
\hline$u_{2}$ & $u_{2}$ & $u_{2}$ & $\alpha w_{1}$ & 0 & $u_{3}+\gamma w_{3}$ & $u_{1}+\beta w_{1}$ & 0 & 0 \\
\hline$u_{3}$ & 0 & $u_{3}$ & 0 & $\gamma w_{1}$ & $\gamma w_{2}$ & 0 & $u_{1}+\beta w_{1}$ & $u_{2}+\alpha w_{2}$ \\
\hline$y$ & 0 & 0 & $\delta_{1} w_{3}+\delta_{2} u_{3}$ & $\delta_{3} w_{2}+\delta_{4} u_{2}$ & $\delta_{5} w_{1}+\delta_{6} u_{1}$ & $\delta_{7} w_{3}+\delta_{8} u_{3}$ & $\delta_{9} w_{2}+\delta_{10} u_{2}$ & $\delta_{11} w_{1}+\delta_{12} u_{1}$ \\
\hline$x$ & 0 & 0 & $\delta_{13} w_{3}+\delta_{14} u_{3}$ & $\delta_{15} w_{2}+\delta_{16} u_{2}$ & $\delta_{17} w_{1}+\delta_{18} u_{1}$ & $\delta_{19} w_{3}+\delta_{20} u_{3}$ & $\delta_{21} w_{2}+\delta_{22} u_{2}$ & $\delta_{23} w_{1}+\delta_{24} u_{1}$ \\
\hline
\end{tabular}

Tabela 3.76: $O \mathfrak{s l}_{3}(k)-2$-módulos $U^{293}=V_{0} \curlywedge\left[V_{0} \curlywedge\left(V_{3} \curlywedge V_{3}^{*}\right)\right]$

Da tabela anterior temos que:

$$
U^{293} \simeq V_{0} \oplus\left[V_{0} \oplus\left(V_{3} \oplus V_{3}^{*}\right)\right] \Longleftrightarrow \delta_{i}=\alpha=\beta=\gamma=0 .
$$


59. Tabela para o $\mathfrak{s l}_{3}(k)-2$-módulo $U^{294}:=V_{0} \curlywedge\left[\left(V_{3} \curlywedge V_{3}^{*}\right) \curlywedge V_{0}\right]$

\begin{tabular}{|c||c|c|c|c|c|c|c|c|}
\hline & $h_{1}$ & $h_{2}$ & $e_{12}$ & $e_{13}$ & $e_{23}$ & $e_{21}$ & $e_{31}$ & $e_{32}$ \\
\hline \hline$b$ & 0 & 0 & 0 & 0 & 0 & 0 & 0 & $b_{3}$ \\
\hline$b_{1}$ & $b_{1}$ & 0 & 0 & 0 & $\delta_{1} b$ & $b_{2}$ & $\delta_{2} b$ & $b_{3}$ \\
\hline$b_{2}$ & $b_{2}$ & $b_{2}$ & $b_{1}$ & $\delta_{3} b$ & 0 & 0 & 0 & $\delta_{8}$ \\
\hline$b_{3}$ & 0 & $b_{3}$ & $\delta_{5} b$ & $b_{1}$ & $b_{2}$ & $\delta_{6} b$ & $\beta b_{3}$ & $\delta_{10} b$ \\
\hline$d_{1}$ & $d_{1}$ & 0 & $d_{2}+\alpha b_{2}$ & $d_{3}+\gamma b_{3}$ & $\delta_{7} b$ & $b_{2}$ & $\delta_{3}$ \\
\hline$d_{2}$ & $d_{2}$ & $d_{2}$ & $\alpha b_{1}$ & $\delta_{9} b$ & $d_{3}+\gamma b_{3}$ & $d_{1}+\beta b_{1}$ & $\delta_{12} b$ \\
\hline$d_{3}$ & 0 & $d_{3}$ & $\delta_{11} b$ & $\gamma b_{1}$ & $\gamma b_{2}$ & $\delta_{12} b$ & $d_{1}+\beta b_{1}$ & $d_{2}+\alpha b_{2}$ \\
\hline$d$ & 0 & 0 & $\delta_{13} b_{3}+\delta_{14} d_{3}$ & $\delta_{15} b_{2}+\delta_{16} d_{2}$ & $\delta_{17} b_{1}+\delta_{18} d_{1}$ & $\delta_{19} b_{3}+\delta_{20} d_{3}$ & $\delta_{21} b_{2}+\delta_{22} d_{2}$ & $\delta_{23} b_{1}+\delta_{24} d_{1}$ \\
\hline
\end{tabular}

Tabela 3.77: $O \mathfrak{s l}_{3}(k)-2-$ módulos $U^{294}:=V_{0} \wedge\left[\left(V_{3} \curlywedge V_{3}^{*}\right) \curlywedge V_{0}\right]$

Da tabela anterior temos que:

$$
U^{294} \simeq V_{0} \oplus\left[\left(V_{3} \oplus V_{3}^{*}\right) \oplus V_{0}\right] \Longleftrightarrow \delta_{i}=\alpha=\beta=\gamma=0 .
$$

60. Tabela para o $\mathfrak{s l}_{3}(k)-2-$ módulo $U^{298}:=V_{0} \curlywedge\left[V_{0} \curlywedge\left(V_{3}^{*} \curlywedge V_{3}\right)\right]$

\begin{tabular}{|c||c|c|c|c|c|c|c|c|}
\hline & $h_{1}$ & $h_{2}$ & $e_{12}$ & $e_{13}$ & $e_{23}$ & $e_{21}$ & $e_{31}$ & $e_{32}$ \\
\hline \hline$v_{1}$ & $v_{1}$ & 0 & $v_{2}$ & $v_{3}$ & 0 & 0 & 0 \\
\hline$v_{2}$ & $v_{2}$ & $v_{2}$ & 0 & 0 & $v_{3}$ & $v_{1}$ & 0 & 0 \\
\hline$v_{3}$ & 0 & $v_{3}$ & 0 & 0 & 0 & 0 & $v_{1}$ & $v_{2}$ \\
\hline$u_{1}$ & $u_{1}$ & 0 & $\alpha v_{2}$ & $\alpha v_{3}$ & 0 & $u_{2}+\gamma v_{2}$ & $u_{3}+\beta v_{3}$ & 0 \\
\hline$u_{2}$ & $u_{2}$ & $u_{2}$ & $u_{1}+\alpha v_{1}$ & 0 & $\gamma v_{3}$ & $\gamma v_{1}$ & 0 & $u_{3}+\beta v_{3}$ \\
\hline$u_{3}$ & 0 & $u_{3}$ & 0 & $u_{1}+\alpha v_{1}$ & $u_{2}+\gamma v_{2}$ & 0 & $\beta v_{1}$ & $\delta_{2}$ \\
\hline$a$ & 0 & 0 & $\delta_{1} v_{3}+\delta_{2} u_{3}$ & $\delta_{3} v_{2}+\delta_{4} u_{2}$ & $\delta_{5} v_{1}+\delta_{6} u_{1}$ & $\delta_{7} v_{3}+\delta_{8} u_{3}$ & $\delta_{9} v_{2}+\delta_{10} u_{2}$ & $\delta_{11} v_{1}+\delta_{12} u_{1}$ \\
\hline$b$ & 0 & 0 & $\delta_{13} v_{3}+\delta_{14} u_{3}$ & $\delta_{15} v_{2}+\delta_{16} u_{2}$ & $\delta_{17} v_{1}+\delta_{18} u_{1}$ & $\delta_{19} v_{3}+\delta_{20} u_{3}$ & $\delta_{21} v_{2}+\delta_{22} u_{2}$ & $\delta_{23} v_{1}+\delta_{24} u_{1}$ \\
\hline
\end{tabular}

Tabela 3.78: $O \mathfrak{s l}_{3}(k)-2-$ módulos $U^{298}:=V_{0} \curlywedge\left[V_{0} \curlywedge\left(V_{3}^{*} \curlywedge V_{3}\right)\right]$

Da tabela anterior temos que:

$$
U^{294} \simeq V_{0} \oplus\left[V_{0} \oplus\left(V_{3}^{*} \oplus V_{3}\right)\right] \Longleftrightarrow \delta_{i}=\alpha=\beta=\gamma=0 .
$$


61. Tabela para o $\mathfrak{s l}_{3}(k)-2-$ módulo $U^{1}:=\left(V_{3}^{*} \curlywedge V_{0}\right) \curlywedge\left(V_{3}^{*} \curlywedge V_{0}\right)$.

Seja $\left\{a_{0}, a_{1}, a_{2}, a_{3}, b_{0}, b_{1}, b_{2}, b_{3}\right\}$ uma base de $U^{1}$, a ação de $\mathfrak{s l}_{3}(k)$ sobre $U^{1}$ é dada pela tabela seguinte.

\begin{tabular}{|c||c|c|c|c|c|c|c|c|}
\hline & $h_{1}$ & $h_{2}$ & $e_{12}$ & $e_{13}$ & $e_{23}$ & $e_{21}$ & $e_{31}$ & $e_{32}$ \\
\hline \hline$a_{0}$ & 0 & 0 & 0 & 0 & 0 & 0 & 0 & 0 \\
\hline$a_{1}$ & $a_{1}$ & 0 & 0 & 0 & $\pi a_{0}$ & $a_{2}$ & $a_{3}$ & $\lambda a_{0}$ \\
\hline$a_{2}$ & $a_{2}$ & $a_{2}$ & $a_{1}$ & $\pi a_{0}$ & 0 & 0 & $\xi a_{0}$ & $a_{3}$ \\
\hline$a_{3}$ & 0 & $a_{3}$ & $\lambda a_{0}$ & $a_{1}$ & $a_{2}$ & $\xi a_{0}$ & 0 & 0 \\
\hline$b_{0}$ & 0 & 0 & $\delta_{1} a_{3}$ & $\delta_{2} a_{2}$ & $\delta_{3} a_{1}$ & $\delta_{4} a_{3}$ & $\delta_{5} a_{2}$ & $\delta_{6} a_{1}$ \\
\hline$b_{1}$ & $b_{1}$ & 0 & $\delta_{7} a_{2}$ & $\delta_{8} a_{3}$ & $\delta_{9} a_{0}+\pi b_{0}$ & $b_{2}+\delta_{10} a_{2}$ & $b_{3}+\delta_{11} a_{3}$ & $\lambda b_{0}+\delta_{12} a_{0}$ \\
\hline$b_{2}$ & $b_{2}$ & $b_{2}$ & $b_{1}+\delta_{13} a_{1}$ & $\pi b_{0}+\delta_{14} a_{0}$ & $\delta_{15} a_{3}$ & $\delta_{16} a_{1}$ & $\xi b_{0}+\delta_{17} a_{0}$ & $b_{3}+\delta_{18} a_{3}$ \\
\hline$b_{3}$ & 0 & $b_{3}$ & $\lambda b_{0}+\delta_{19} a_{0}$ & $b_{1}+\delta_{20} a_{1}$ & $b_{1}+\delta_{21} a_{2}$ & $\xi b_{0}+\delta_{22} a_{0}$ & $\delta_{23} a_{1}$ & $\delta_{24} a_{3}$ \\
\hline
\end{tabular}

Tabela 3.79: $O \mathfrak{s l}_{3}(k)-2-m o ́ d u l o ~ U^{1}:=\left(V_{3}^{*} \curlywedge V_{0}\right) \curlywedge\left(V_{3}^{*} \curlywedge V_{0}\right)$.

Dessa tabela, podemos inferir que $U^{1}$ cinde se, e só se, $\delta_{i}=0$ para $i=1,2, \ldots, 24$, isto é,

$$
U^{1} \simeq\left(V_{3}^{*} \curlywedge V_{0}\right) \oplus\left(V_{3}^{*} \curlywedge V_{0}\right) \Longleftrightarrow \delta_{i}=0 \text { para } i=1, \ldots, 24
$$

e, também de 3.2.2.3 temos, $V_{3}^{*} \curlywedge V_{0}$ cinde $\Longleftrightarrow \pi=\lambda=\xi=0$.

Portanto:

$$
U^{1} \simeq\left(V_{3}^{*} \oplus V_{0}\right) \oplus\left(V_{3}^{*} \oplus V_{0}\right) \Longleftrightarrow \delta_{i}=0=\pi=\lambda=\xi \text { para } i=1, \ldots, 24 .
$$

\begin{tabular}{|c||c|c|c|c|c|c|c|c|}
\hline & $h_{1}$ & $h_{2}$ & $e_{12}$ & $e_{13}$ & $e_{23}$ & $e_{21}$ & $e_{31}$ & $e_{32}$ \\
\hline \hline$a_{0}$ & 0 & 0 & 0 & 0 & 0 & 0 & 0 & 0 \\
\hline$a_{1}$ & $a_{1}$ & 0 & 0 & 0 & $\pi a_{0}$ & $a_{2}$ & $a_{3}$ & $\lambda a_{0}$ \\
\hline$a_{2}$ & $a_{2}$ & $a_{2}$ & $a_{1}$ & $\pi a_{0}$ & 0 & 0 & $\xi a_{0}$ & $a_{3}$ \\
\hline$a_{3}$ & 0 & $a_{3}$ & $\lambda a_{0}$ & $a_{1}$ & $a_{2}$ & $\xi a_{0}$ & 0 & 0 \\
\hline$b_{0}$ & 0 & 0 & 0 & 0 & 0 & 0 & 0 & 0 \\
\hline$b_{1}$ & $b_{1}$ & 0 & 0 & 0 & $\pi b_{0}$ & $b_{2}$ & $b_{3}$ & $\lambda b_{0}$ \\
\hline$b_{2}$ & $b_{2}$ & $b_{2}$ & $b_{1}$ & $\pi b_{0}$ & 0 & 0 & $\xi b_{0}$ & $b_{3}$ \\
\hline$b_{3}$ & 0 & $b_{3}$ & $\lambda b_{0}$ & $b_{1}$ & $b_{1}$ & $\xi b_{0}$ & 0 & 0 \\
\hline
\end{tabular}

Tabela 3.80: $O \mathfrak{s l}_{3}(k)-2-m o ́ d u l o ~ U^{1}:=\left(V_{3}^{*} \curlywedge V_{0}\right) \oplus\left(V_{3}^{*} \curlywedge V_{0}\right)$.

Finalizamos este capítulo, demonstrando o seguinte teorema, o qual da um critério que posteriormente nós permitirá decidir quando o $k$-espaço vetorial $\mathfrak{s l}_{3}(k) \oplus k t_{3} \oplus U^{i}$ tem estrutura de $k$-álgebra, para todo $1 \leq i \leq 365$. 
Teorema 3.2. Seja $U^{i}$ qualquer $\mathfrak{s l}_{3}(k)$-2-módulo de dimensão 8 listado na seção 3.3, para $1 \leq i \leq 365$. Então as seguintes proposições são equivalentes.

1. Existem $\mathfrak{s l}_{3}(k)$-2-submódulos $Q_{1}, Q_{2}, P_{3}$ e $P_{4}$ de $U^{i}$ tais que:

a. $Q_{2} \subset Q_{1}, Q_{1} / Q_{2} \simeq V_{3}$ e $Q_{1} \oplus Q=U^{i}$, para algum $\mathfrak{s l}_{3}(k)$-submódulos $Q$ de $U^{i}$.

b. $P_{4} \subset P_{3}, P_{3} / P_{4} \simeq V_{3}^{*}$ e $P_{3} \oplus P=U^{i}$, para algum $\mathfrak{s l}_{3}(k)$-submódulos $P$ de $U^{i}$.

2. Existe um $\mathfrak{s l}_{3}(k)$-morfismo $0 \neq \varphi: \Lambda^{2}\left(U^{i}\right) \rightarrow \mathfrak{s l}_{3}(k) \oplus k t_{3}$.

Demonstração. (1.) $\Rightarrow\left(2\right.$.). Seja $U$ qualquer $\mathfrak{s l}_{3}(k)$-2-módulos de dimensão 8 enumerado na seção 3.3. satisfazendo a condição 1. do teorema. De a. e b. temos a seguintes sequência exata curtas

$$
0 \rightarrow Q_{2} \hookrightarrow Q_{1} \rightarrow Q_{1} / Q_{2} \rightarrow 0 \text { e } 0 \rightarrow P_{4} \hookrightarrow P_{3} \rightarrow P_{3} / P_{4} \rightarrow 0
$$

portanto, podemos construir as sequências exatas

$$
0 \rightarrow Q_{2} \oplus Q \hookrightarrow Q_{1} \oplus Q \rightarrow Q_{1} / Q_{2} \rightarrow 0 \quad \text { e } 0 \rightarrow P_{4} \oplus P \hookrightarrow P_{3} \oplus P \rightarrow P_{3} / P_{4} \rightarrow 0 .
$$

Assim, pelo teorema de isomorfismo de $\mathfrak{s l}_{3}(k)$-módulos se obtêm:

$$
\left(Q_{1} \oplus Q\right) /\left(Q_{2} \oplus Q\right) \simeq Q_{1} / Q_{2} \text { e }\left(P_{3} \oplus P\right) /\left(P_{4} \oplus P\right) \simeq P_{3} / P_{4}
$$

Como $Q_{1} \oplus Q=U=P_{3} \oplus P, Q_{1} / Q_{2} \simeq V_{3}$ e, $P_{3} / P_{4} \simeq V_{3}^{*}$, então se tem os seguintes $\mathfrak{s l}_{3}(k)$-isomorfismos: $U /\left(Q_{2} \oplus Q\right) \simeq V_{3}$ e $U /\left(P_{4} \oplus P\right) \simeq V_{3}^{*}$. Assim, temos o seguinte $\mathfrak{s l}_{3}(k)$-isomorfismo de módulos:

$$
\sigma: U /\left(Q_{2} \oplus Q\right) \otimes_{k} U /\left(P_{4} \oplus P\right) \simeq V_{3} \otimes V_{3}^{*} \simeq \mathfrak{s l}_{3}(k) \oplus \mathfrak{z}\left(\mathfrak{g l}_{3}(k)\right) \simeq \mathfrak{s l}_{3}(k) \oplus k t_{3} .
$$

Agora, os $\mathfrak{s l}_{3}(k)$-morfismos quocientes

$$
p_{1}: U \rightarrow U /\left(Q_{2} \oplus Q\right) \text { e } p_{2}: U \rightarrow U /\left(P_{4} \oplus P\right)
$$

induzem o $\mathfrak{s l}_{3}(k)$-morfismo de módulos

$$
p_{1} \otimes p_{2}: U \otimes_{k} U \rightarrow U /\left(Q_{2} \oplus Q\right) \otimes_{k} U /\left(P_{4} \oplus P\right)
$$

definido por: $\left(p_{1} \otimes p_{2}\right)(u \otimes v):=p_{1}(u) \otimes p_{2}(v)$ para todo $u, v \in U$.

Portanto, existe um $\mathfrak{s l}_{3}(k)$-morfismo $\varphi$ definido pela seguinte composição de $\mathfrak{s l}_{3}(k)$ morfismo

$$
\varphi: \Lambda^{2}(U) \stackrel{\alpha_{2, U}}{\longrightarrow} U \otimes_{k} U \stackrel{p_{1} \otimes p_{2}}{\longrightarrow} U /\left(Q_{2} \oplus Q\right) \otimes_{k} U /\left(P_{4} \oplus P\right) \stackrel{\sigma}{\rightarrow} \mathfrak{s l}_{3}(k) \oplus k t_{3}
$$

onde $\alpha_{2, U}$ é definido como o exemplo 1.21.3.

$(2.) \Rightarrow(1$.$) . Esboço da prova. O primeiro passo para abordar a prova é pegar cada$ umo dos módulos $U^{i}$ de dimensão 8 enumerados na seção 3.3 com suas respectivas 
tabelas de $\mathfrak{s l}_{3}(k)$-ação, e provar esta implicação para cada um desses $365, \mathfrak{s l}_{3}(k)-2$ módulo $U^{i}$, argumentando por contradição, isto é, supor que existe um $\mathfrak{s l}_{3}(k)$-morfismo $\varphi: \Lambda^{2}\left(U^{i}\right) \rightarrow \mathfrak{s l}_{3}(k) \oplus k t_{3}$ não nulo para cada $i \in I_{365}$, e que não ocorre a condição (1.) do teorema. Por exemplo, suponhamos que existe um $\mathfrak{s l}_{3}(k)$-morfismo $0 \neq \varphi: \Lambda^{2}\left(U^{i}\right) \rightarrow$ $\mathfrak{s l}_{3}(k) \oplus k t_{3}$ para cada $i \in I_{365}$ e não ocurre a condição a. do teorema 3.2. Vejamos que acontece para $i=1$ : peguemos o $\mathfrak{s l}_{3}(k)-2$-módulo $U^{1}:=\left(V_{3}^{*} \oplus V_{0}\right) \oplus\left(V_{3}^{*} \oplus V_{0}\right)$ ver tabelas 3.80, 3.3, e suponhamos que existe um $\mathfrak{s l}_{3}(k)$-morfismo $0 \neq \varphi: \Lambda^{2}\left(U^{1}\right) \rightarrow \mathfrak{s l}_{3}(k) \oplus k t_{3}$, e $U^{1}$ não cumpre a condição a. do teorema 3.2. Pelo exemplo 1.21.5 tem-se:

$$
\begin{aligned}
\Lambda^{2}\left(U^{1}\right) \simeq & \Lambda^{2}\left(V_{0} \oplus V_{3}^{*}\right) \oplus\left(\left(V_{0} \oplus V_{3}^{*}\right) \otimes\left(V_{0} \oplus V_{3}^{*}\right)\right) \oplus \Lambda^{2}\left(V_{0} \oplus V_{3}^{*}\right) \\
\simeq & \left(V_{0} \wedge V_{0}\right) \oplus\left(V_{0} \otimes_{k} V_{3}^{*}\right) \oplus\left(V_{3}^{*} \wedge V_{3}^{*}\right) \oplus\left(V_{0} \otimes_{k} V_{0}\right) \oplus\left(V_{0} \otimes_{k} V_{3}^{*}\right) \\
& \oplus\left(V_{3}^{*} \otimes_{k} V_{0}\right) \oplus\left(V_{3}^{*} \otimes_{k} V_{3}^{*}\right) \oplus\left(V_{0} \wedge V_{0}\right) \oplus\left(V_{0} \otimes_{k} V_{3}^{*}\right) \oplus\left(V_{3}^{*} \wedge V_{3}^{*}\right) \\
\simeq & \left(V_{0} \otimes_{k} V_{3}^{*}\right) \oplus\left(V_{3}^{*} \wedge V_{3}^{*}\right) \oplus\left(V_{0} \otimes_{k} V_{0}\right) \oplus\left(V_{0} \otimes_{k} V_{3}^{*}\right) \\
& \oplus\left(V_{3}^{*} \otimes_{k} V_{0}\right) \oplus\left(V_{3}^{*} \otimes_{k} V_{3}^{*}\right) \oplus\left(V_{0} \otimes_{k} V_{3}^{*}\right) \oplus\left(V_{3}^{*} \wedge V_{3}^{*}\right) .
\end{aligned}
$$

Logo, temos os seguintes $\mathfrak{s l}_{3}(k)$-submódulos de $\Lambda^{2}\left(U^{1}\right)$ :

- $W_{1}:=V_{0} \otimes_{k} V_{3}^{*} \simeq V_{3}^{*} \otimes V_{0} \simeq V_{3}^{*}$.

- $W_{2}:=V_{3}^{*} \wedge V_{3}^{*} \simeq V_{3}^{*}$.

- $W_{3}:=V_{3}^{*} \otimes_{k} V_{3}^{*}$.

- $W_{4}:=V_{0} \otimes_{k} V_{0} \simeq V_{0}$

Vejamos como está definida $\left.\varphi\right|_{W_{i}}$ para todo $i=1,2,3,4$.

Tem-se, $\mathfrak{s l}_{3}(k)$-morfismo $0 \neq \varphi: \Lambda^{2}\left(U^{1}\right) \rightarrow \mathfrak{s l}_{3}(k) \oplus k t_{3}$, então o $\mathfrak{s l}_{3}(k)$-morfismo $p \circ \varphi: \Lambda^{2}\left(U^{1}\right) \rightarrow k t_{3}$ é sobrejetor (já que $k t_{3}$ é um $\mathfrak{s l}_{3}(k)$-módulo irredutível) onde $p: \mathfrak{s l}_{3}(k) \oplus k t_{3} \rightarrow k t_{3}$, (ou também pode escolher $p: \mathfrak{s l}_{3}(k) \oplus k t_{3} \rightarrow \mathfrak{s l}_{3}(k)$ ) e $\operatorname{Ker}(p \circ \varphi)=\varphi^{-1}\left(\mathfrak{s l}_{3}(k)\right)$. Portanto, $\Lambda^{2}\left(U^{1}\right) / \varphi^{-1}\left(\mathfrak{s l}_{3}(k)\right) \simeq k t_{3}$, e $\operatorname{assim} \Lambda^{2}\left(U^{1}\right) / \varphi^{-1}\left(\mathfrak{s l}_{3}(k)\right)$ é um $\mathfrak{s l}_{3}(k)$-módulo irredutível. Então $\varphi^{-1}\left(\mathfrak{s l}_{3}(k)\right)$ é um $\mathfrak{s l}_{3}(k)$-submódulo maximal de $\Lambda^{2}\left(U^{1}\right)$, e da tabela 3.3 se pode ver que $W_{i} \subseteq \varphi^{-1}\left(\mathfrak{s l}_{3}(k)\right)$, já que cada elemento da base de $V_{3}^{*} \wedge V_{3}^{*}$ tem peso $(1,0),(1,1),(0,1), p \circ \varphi$ é um $\mathfrak{s l}_{3}(k)$-morfismo e, $k t_{3} \cdot \mathfrak{s l}_{3}(k)=0$. Assim, $\varphi\left(W_{i}\right) \subseteq \mathfrak{s l}_{3}(k)$ para $i=1,2,3,4$. Logo, para $i=1,2,4,\left.\varphi\right|_{W_{i}}: W_{i} \rightarrow \mathfrak{s l}_{3}(k)$ é um $\mathfrak{s l}_{3}(k)$-isomorfismo, já que $W_{i}$ e $\mathfrak{s l}_{3}(k)$ são $\mathfrak{s l}_{3}(k)$-módulo irredutíveis, o qual é absurdo. Portanto, pelo lema de Schur (lema 1.19), $\left.\varphi\right|_{W_{i}}=0$ pata todo $i=1,2,4$. Para $i=3$, temos que $W_{2}$ é um $\mathfrak{s l}_{3}(k)$-submódulo de $W_{3}$, então $\left.\varphi\right|_{W_{3}}\left(W_{2}\right)=0$, já que $\left.\varphi\right|_{W_{2}}=0$, assim $W_{2} \subseteq \operatorname{Ker}\left(\left.\varphi\right|_{W_{3}}\right)$. Por outro lado, $W_{3} / \operatorname{Ker}\left(\left.\varphi\right|_{W_{3}}\right) \simeq \mathfrak{s l}_{3}(k)$, então $\operatorname{Ker}\left(\left.\varphi\right|_{W_{3}}\right) \simeq k$. Portanto, $W_{2}=k$ ou $W_{2}=0$, o qual é absurdo, e assim $\left.\varphi\right|_{W_{3}}=0$. Daí se segue que $\varphi \equiv 0$. Uma contradição. Pelo mesmo argumento anterior, podemos ver que o mesmo acontece para os $\mathfrak{s l}_{3}(k)$-2-módulos $U^{i}$ para $i=3,9,11,43,44,49,50$, $51,52,55,56,61,62,63,64,68,73,74,75,76,79,80,85,86,87,88,91,92,97$, $98,99,100,104,109,110,111,112,115,116,121,122,123,124,127,128,133,134$, $135,136,147,148,151,152,155,156,161,162,165,166,171,172,173,174,175,176$, 
181, 182, 187, 188, 191, 192, 195, 196, 199, 200, 202, 205, 206, 208, 211, 212, 214, $217,218,220,224,225,227,230,231,234,237,238,240,243,244,246,249,250$, $261,262,265,266,269,270,275,276,279,280,285,286,287,288,289,290,295,296$, $301,302,305,306,309,310,313,314,316,319,320,322,325,326,328,331,332,334$, $338,339,341,344,345,348,351,352,354,357,358,360,363,364,365$ já que eles não satisfazem automaticamente a condição a. do teorema 3.2. Agora, consideremos os $\mathfrak{s l}_{3}(k)$-2-módulos $U^{i}$ para $i=6,8,34,130,252,281,303,355$. Vamos ver que nenhum deles podem cindir, caso contrário satisfazem a condição a. do teorema. De fato, se $U^{6}=\left(V_{3} \curlywedge V_{0}\right) \oplus\left(V_{3} \curlywedge V_{0}\right)$ então pegando $Q_{1}:=\left(V_{3}<V_{0}\right), Q_{2}:=V_{0}$ e $Q:=\left(V_{3} \curlywedge V_{0}\right)$ temos que $Q_{1} / Q_{2} \simeq V_{3}$ e $Q_{1} \oplus Q=U^{6}$. Portanto se $U^{6}$ cinde tem-se que cumprem a. o qual é uma contradição. Assim que, $U^{6} \neq\left(V_{3} \curlywedge V_{0}\right) \oplus\left(V_{3} \curlywedge V_{0}\right)$. O mesmo acontece com $U^{8}$, isto é, se $U^{8}=\left(V_{0} \wedge V_{3}\right) \oplus\left(V_{3} \wedge V_{0}\right)$ e pegamos $Q_{1}:=V_{3} \wedge V_{0}, Q_{2}:=V_{0}$ e, $Q:=V_{0} \wedge V_{3}$ temos que $Q_{1} / Q_{2} \simeq V_{3}$ e $Q \oplus Q_{1}=U^{8}$. Para $U^{34}=V_{3} \oplus\left[V_{0} \oplus\left(V_{3} \wedge V_{0}\right)\right]$ com $Q_{1}:=V_{3} \wedge V_{0}, Q_{2}:=V_{0}$ e, $Q:=V_{3} \oplus V_{0}$ temos que $Q_{1} / Q_{2} \simeq V_{3}$ e $Q \oplus Q_{1}=U^{34}$, e assim $U^{34}$ cumprem a., portanto $U^{34} \neq V_{3} \oplus\left[V_{0} \oplus\left(V_{3} \wedge V_{0}\right)\right]$.

$U^{130} \neq\left(V_{0}<V_{0}\right) \oplus\left(V_{0} \oplus V_{0}\right) \oplus\left(V_{3}<V_{0}\right)$, do contrario cumprem a., já que se $Q_{1}:=V_{3}<V_{0}$, $Q_{2}:=V_{0}$ e $Q:=\left(V_{0} \wedge V_{0}\right) \oplus\left(V_{0} \oplus V_{0}\right)$ tem-se $Q_{1} / Q_{2} \simeq V_{3}$ e $Q \oplus Q_{1}=U^{130}$.

$\left.U^{252} \neq\left(V_{0} \oplus V_{3}\right) \oplus\left(V_{3}<V_{0}\right)\right]$, caso contrário satisfaz a condição a., com efeito, pegando $Q_{1}:=V_{3}\left\langle V_{0}, Q_{2}:=V_{0}\right.$ e $Q:=V_{3} \oplus V_{0}$ obtemos $Q_{1} / Q_{2} \simeq V_{3}$ e $Q \oplus Q_{1}=U^{252}$.

Quando $U^{281}=\left(V_{0} \oplus V_{0}\right) \oplus\left(V_{0} \curlywedge V_{0}\right) \oplus\left(V_{3} \wedge V_{0}\right)$ então, para $Q_{1}:=V_{3} \wedge V_{0}, Q_{2}:=V_{0}$ e $Q:=\left(V_{0} \oplus V_{0}\right) \oplus\left(V_{0} \wedge V_{0}\right)$ tem-se que $Q_{1} / Q_{2} \simeq V_{3}$ e $Q \oplus Q_{1}=U^{281}$, logo $U^{281}$ dessa forma cumprem a condição a. do teorema, o qual não pode ser. O mesmo acontece para $U^{303}=\left(V_{0} \oplus V_{0}\right) \oplus\left(V_{0}<V_{0}\right) \oplus\left(V_{3}<V_{0}\right)$.

Se $U^{355}=\left(V_{0} \oplus V_{0} \oplus V_{0} \oplus V_{0}\right) \oplus\left(V_{3} \wedge V_{0}\right)$ e $Q_{1}:=V_{3} \wedge V_{0}, Q_{2}:=V_{0}, Q:=V_{0}^{\oplus 4}$ temos que $U^{355}$ cumprem a. do teorema. Uma contradição. Logo, para $i=6,8,34$, 130, 252, 281, 303, 355 observamos que $V_{3} \prec V_{0}$ é $\mathfrak{s l}_{3}(k)$-2-submódulos de $U^{i}$, e assim $\left(V_{3} \wedge V_{0}\right) \wedge\left(V_{3} \wedge V_{0}\right)$ é um $\mathfrak{s l}_{3}(k)$-2-submódulos $\Lambda^{2}\left(U^{i}\right)$, mas

$\left(V_{3} \wedge V_{0}\right) \wedge\left(V_{3} \wedge V_{0}\right) \simeq V_{3} \wedge V_{0} \simeq V_{3}$, portanto o $\mathfrak{s l}_{3}(k)$-morfismo sobrejetor $p \circ \varphi \circ \iota$ : $V_{3} \rightarrow \mathfrak{s l}_{3}(k)$ é um $\mathfrak{s l}_{3}(k)$-isomorfismo, o qual é absurdo. Se continuamos procurando os submódulos de $\Lambda^{2}\left(U^{i}\right)$ (note que não é possivel achar uma cópia de $V_{3}^{*}$ em $\Lambda^{2}\left(U^{i}\right)$ ) e argumentamos como no caso $i=1$ achamos que $\varphi=0$. Agora, seja $i=14,16,17,18$, $21,22,23,24,30,36,102,126,132,251,255,256,278,283,304,356,359$. Para estes $i$ temos que $U^{i}$ não cinde. De fato, para $i=14, U^{14} \neq\left(V_{3} \wedge V_{0}\right) \oplus\left(V_{0} \wedge V_{3}\right)$ do contrário, se $Q_{1}:=V_{3} \wedge V_{0}, Q_{2}:=V_{0}$ e $Q:=V_{0} \wedge V_{3}$ então $Q_{1} / Q_{2} \simeq V_{3}$ e $Q \oplus Q_{1}=U^{14}$, portanto cumpre a., o qual é absurdo.

Se $U^{16}=\left(V_{0} \wedge V_{3}\right) \oplus\left(V_{0} \wedge V_{3}\right), Q_{1}:=V_{3}, Q_{2}:=0$ e, $Q:=\left(V_{0} \wedge V_{3}\right) \oplus V_{0}$ então se cumpre a. do teorema, o qual é absurdo. Quando $U^{17}=\left[V_{3} \wedge\left(V_{0} \wedge V_{0}\right)\right] \oplus V_{3}, Q_{1}:=V_{3}$, $Q_{2}:=0$ e, $Q:=V_{3} \wedge\left(V_{0} \wedge V_{0}\right)$ então se cumpre a. do teorema, o qual é absurdo.

Se $U^{18}=\left[\left(V_{0} \prec V_{0}\right) \curlywedge V_{3}\right] \curlywedge V_{3}, Q_{1}:=V_{3}, Q_{2}:=0$ e, $Q:=\left(V_{0} \prec V_{0}\right) \curlywedge V_{3}$, então se cumpre a. do teorema, o qual é absurdo.

Quando $U^{21}=\left[\left(V_{3} \wedge V_{0}\right) \wedge V_{0}\right] \oplus V_{3}, Q_{1}:=V_{3}, Q_{2}:=0$ e, $Q:=\left(V_{3} \wedge V_{0}\right) \curlywedge V_{0}$, então se cumpre a. do teorema, o qual é absurdo.

Se $U^{22}=\left[V_{0} \curlywedge\left(V_{3} \curlywedge V_{0}\right)\right] \oplus V_{3}, Q_{1}:=V_{3}, Q_{2}:=0$ e, $Q:=V_{0} \curlywedge\left(V_{3} \curlywedge V_{0}\right)$ então se 
cumpre a. do teorema, o qual é absurdo.

Se $U^{23}=\left[\left(V_{0} \wedge V_{3}\right) \wedge V_{0}\right] \oplus V_{3}, Q_{1}:=V_{3}, Q_{2}:=0$ e, $Q:=\left(V_{0}<V_{3}\right) \curlywedge V_{0}$, então se cumpre a. do teorema, o qual é absurdo.

Se $U^{24}=\left[V_{0} \curlywedge\left(V_{0} \curlywedge V_{3}\right)\right] \oplus V_{3}, Q_{1}:=V_{3}, Q_{2}:=0$ e, $Q:=V_{0} \curlywedge\left(V_{0} \curlywedge V_{3}\right)$, então se cumpre a. do teorema, o qual é absurdo.

Quando $U^{30}=V_{3} \oplus\left[\left(V_{0} \wedge V_{0}\right) \curlywedge V_{3}\right], Q_{1}:=V_{3}, Q_{2}:=0$ e, $Q:=\left(V_{0} \wedge V_{0}\right) \curlywedge V_{3}$, então se cumpre a. do teorema, o qual é absurdo.

Se $U^{36}=V_{3} \oplus\left[V_{0} \curlywedge\left(V_{0} \wedge V_{3}\right)\right], Q_{1}:=V_{3}, Q_{2}:=0$ e, $Q:=\left(V_{0} \curlywedge\left(V_{0} \curlywedge V_{3}\right)\right.$, então se cumpre a. do teorema, o qual é absurdo.

Quando $U^{102}=\left(V_{0} \wedge V_{0}\right) \oplus\left(V_{3} \wedge V_{3}\right), Q_{1}:=V_{3} \wedge V_{3}, Q_{2}:=V_{3}$ e, $Q:=\left(V_{0} \wedge V_{0}\right)$ então se cumpre a. do teorema, o qual é absurdo.

Quando $U^{126}=\left(V_{0} \wedge V_{0}\right) \oplus\left[V_{0} \oplus\left(V_{0} \wedge V_{0}\right) \oplus V_{3}\right], Q_{1}:=V_{3}, Q_{2}:=0 \mathrm{e}$,

$Q:=\left(V_{0} \wedge V_{0}\right) \oplus\left(V_{0}<V_{0}\right) \oplus V_{0}$, então se cumpre a. do teorema, o qual é absurdo. Portanto $U^{126} \neq\left(V_{0}<V_{0}\right) \oplus\left[V_{0} \oplus\left(V_{0}<V_{0}\right) \oplus V_{3}\right]$.

Se $U^{132}=\left(V_{0} \curlywedge V_{0}\right) \oplus\left(V_{0} \oplus V_{0}\right) \oplus\left(V_{0} \curlywedge V_{3}\right), Q_{1}=V_{3}, Q_{2}=0$ e,

$Q=\left(V_{0} \wedge V_{0}\right) \oplus\left(V_{0} \oplus V_{0}\right)$ então se cumpre a. do teorema, o qual é absurdo. Portanto $U^{132} \neq\left(V_{0} \wedge V_{0}\right) \oplus\left(V_{0} \oplus V_{0}\right) \oplus\left(V_{0} \wedge V_{3}\right)$.

Se $U^{251}=V_{0} \oplus\left(V_{3} \wedge V_{0}\right) \oplus V_{3}, Q_{1}:=V_{3} \wedge V_{0}, Q_{2}:=V_{0}$ e, $Q=V_{0} \oplus V_{3}$, então se cumpre a. do teorema, o qual é absurdo. Portanto $U^{251} \neq V_{0} \oplus\left(V_{3} \wedge V_{0}\right) \oplus V_{3}$.

Se $U^{255}, U^{256}=V_{0} \oplus\left(V_{0} \wedge V_{3}\right) \oplus V_{3}, Q_{1}:=V_{3}, Q_{2}:=0$ e, $Q=V_{0} \oplus V_{3}$ então se cumpre a. do teorema, o qual é absurdo. Portanto, $U^{255}, U^{256} \neq V_{0} \oplus\left(V_{0} \wedge V_{3}\right) \oplus V_{3}$. Se $U^{278}=V_{0} \oplus\left[\left(V_{0} \curlywedge V_{0}\right) \oplus\left(V_{0} \curlywedge V_{0}\right) \curlywedge V_{3}, Q_{1}=V_{3}, Q_{2}=0\right.$ e,

$Q=\left(V_{0} \curlywedge V_{0}\right) \oplus\left(V_{0} \curlywedge V_{0}\right) \oplus V_{0}$ então se cumpre a. do teorema, o qual é absurdo. Portanto $U^{278} \neq V_{0} \oplus\left[\left(V_{0} \wedge V_{0}\right) \oplus\left(V_{0} \wedge V_{0}\right) \wedge V_{3}\right.$

Se $U^{283}, U^{304}, U^{356}, U^{359}=V_{0}^{\oplus 4} \oplus V_{3}, Q_{1}:=V_{3}, Q_{2}:=0$ e, $Q:=V_{0}^{\oplus 4}$ então se cumpre a. do teorema, o qual é absurdo. Portanto $U^{283}, U^{304}, U^{356}, U^{359} \neq V_{0}^{\oplus 4} \oplus V_{3}$.

Observe-se que, $V_{3}$ é $\mathfrak{s l}_{3}(k)$-2-submódulos de $U^{i}$ e assim $V_{3} \wedge V_{3}$ é um $\mathfrak{s l}_{3}(k)$-2-submódulos $\Lambda^{2}\left(U^{i}\right)$, para todo $i=14,16,17,18,21,22,23,24,30,36,102,126,132,251,255,256$, $278,283,304,356,359$, portanto o $\mathfrak{s l}_{3}(k)$-morfismo sobrejetor $p \circ \varphi \circ \iota: V_{3} \wedge V_{3} \rightarrow \mathfrak{s l}_{3}(k)$ é um $\mathfrak{s l}_{3}(k)$-isomorfismo, ja que $V_{3} \wedge V_{3} \simeq V_{3}$, o qual é uma contradição, assim que novamente considerando os submódulos (que não são isomorfos a $V_{3}^{*}$ ) de essos $\Lambda^{2}\left(U^{i}\right)$ vamos ter alguns contradições. Para os $\mathfrak{s l}_{3}(k)$-2-módulos $U^{i}$ para todo $i=29,33,34,35$, $36,66,69,70,71,72,77,78,81,82,83,84,89,90,93,94,95,96,105,106,107,108$, $113,114,117,118,119,120,125,129,131$ 137, 138, 140, 141, 153, 154, 157, 158, 158, 160, 163, 164, 167, 168, 169, 170, 177, 183, 185, 186, 189, 190, 193, 194, 197, 198, 201, 203, 204, 207, 209, 210, 213, 215, 216, 219, 221, 222, 223, 226, 228, 229, 232, 233, 235, 236, 239, 241, 242, 245, 247, 248, 267, 268, 271, 272, 273, 274, 277, 282, 284, 297, 299, $300,307,308,311,312,315,317,318,321,323,324,327,329,330,333,335,337,340$, $342,343,346,347,349,350,353,361,362$, tem-se que eles não cindem, caso contrário se cumpre a condição a. do teorema. De fato, os módulos $U^{29} \neq V_{3} \oplus\left(V_{3} \wedge\left(V_{0} \wedge V_{0}\right)\right)$, $U^{33} \neq V_{3} \oplus\left(\left(V_{3} \wedge V_{0}\right) \curlywedge V_{0}\right), U^{34} \neq V_{3} \oplus\left(V_{0} \curlywedge\left(V_{3} \curlywedge V_{0}\right)\right), U^{35} \neq V_{3} \oplus\left(\left(V_{0}<V_{3}\right) \curlywedge V_{0}\right)$, $U^{36} \neq V_{3} \oplus\left(V_{0} \wedge\left(V_{0} \wedge V_{3}\right)\right), U^{66} \neq\left(V_{3} \oplus V_{3}\right) \oplus\left(V \wedge V_{0}\right)$, caso contrário com $Q_{1}:=V_{3}$, $Q_{2}:=0$ e $Q$ o complementar de $V_{3}$ se satisfaze a. do teorema. Tambén os módulos 
$U^{69}, U^{70}, U^{81}, U^{82}, U^{93}, U^{94}, U^{105}, U^{106}, U^{117}, U^{118}, U^{129}, U^{137}, U^{138}, U^{157}, U^{158}$, $U^{167}, U^{168}, U^{185} U^{189}, U^{193}, U^{197}, U^{203}, U^{209}, U^{215}, U^{221}, U^{222}, U^{228}, U^{235}, U^{241}$, $U^{242}, U^{247}, U^{271}, U^{272}, U^{282}, U^{299} U^{307}, U^{311}, U^{317}, U^{323}, U^{329}, U^{335}, U^{342}, U^{349}$ $U^{361} \neq\left(V_{0} \wedge V_{0}\right) \oplus\left(V_{0} \wedge V_{0}\right) \oplus\left(V_{3} \wedge V_{0}\right)$, caso contrário, com $Q_{1}:=V_{3} \wedge V_{0}, Q_{2}:=V_{0}$ e $Q:=\left(V_{0} \wedge V_{0}\right)^{\oplus 2}$ tem-se a. do teorema. Agora, $U^{i} \neq V_{3} \oplus V_{0}^{\oplus 5}$ para todo $i=71$, $72,77,83,84,89,90,93,95,96,107,108,113,114,119,120,125,131$ 140,141, 153, 154, 159, 160, 163, 164, 169, 170, 177, 183, 186, 190, 194, 198, 201, 204, 207, 210, 213, 216, 219, 223, 226, 229, 232, 233, 236, 239, 242, 245, 248, 267, 268, 273, 274, 277, 284, $297,300,308,312,315,318,321,324,327,330,333,337,340,343,346,347,350,353$, 362 , porque se temos a igualdade, e $Q_{1}:=V_{3}, Q_{2}:=0$ e $Q:=V_{0}^{\oplus 5}$ temos a condição a. do teorema. Finalmente, consideremos os $\mathfrak{s l}_{3}(k)$-2-módulos $U^{i}$ para $i=2,4,5,7,10$, $12,13,15,19,20,25,26,27,28,31,32,37,38,3940,41,42,45,46,47,48,53,54$, $57,58,59,60,65,67,101,103,138,139,140,143,144,145,148,149,178,179,180$, 184, 253, 254, 257, 258, 259, 260, 263, 264, 292, 293, 294 298, cujas tabelas são dadas na seção 3.3.1. Como nos casos anteriores, estos módulos não podem cindir, porque se eles cindem, satisfazem a condição a. do teorema, e isso contradiz a hipótese. De fato, na seção 4.1.1.2 do capítulo 4 é provado que se $U^{i}$ cinde para todo $i$, então eles cumprem a condição 1 do teorema. Agora, os módulos $U^{2}, U^{7}, U^{38}$ tem a $V_{3}^{*} \wedge V_{0}$ como submódulo, logo $\left(V_{3}^{*} \wedge V_{0}\right) \wedge\left(V_{3}^{*} \wedge V_{0}\right)$ é submódulo irredutível de $\Lambda^{2}\left(U^{i}\right)$ para todo $i=2,7,38$ já que $\left(V_{3}^{*} \wedge V_{0}\right) \wedge\left(V_{3}^{*} \wedge V_{0}\right) \simeq V_{3}^{*}$. Portanto o $\mathfrak{s l}_{3}(k)$-morfismo sobrejetor $p \circ \varphi \circ \iota: \Lambda^{2}\left(V_{3}^{*}<V_{0}\right) \rightarrow \mathfrak{s l}_{3}(k)$ é um $\mathfrak{s l}_{3}(k)$-isomorfismo. Uma contradição. Também $V_{3} \wedge V_{0}$ é um $\mathfrak{s l}_{3}(k)$-2-submódulos de $U^{i}$ e assim $\left(V_{3} \wedge V_{0}\right) \wedge\left(V_{3} \wedge V_{0}\right) \simeq V_{3}$ é um $\mathfrak{s l}_{3}(k)$-2-submódulos irreductível de $\Lambda^{2}\left(U^{i}\right)$, para todo $i=4,5,58,254$. Logo $p \circ \varphi \circ \iota: \Lambda^{2}\left(V_{3} \wedge V_{0}\right) \rightarrow \mathfrak{s l}_{3}(k)$ é um $\mathfrak{s l}_{3}(k)$-isomorfismo. Uma contradição. $V_{3}$ é um $\mathfrak{s l}_{3}(k)$-2-submódulo de $U^{i}$ e assim $V_{3} \wedge V_{3} \simeq V_{3}$ é um $\mathfrak{s l}_{3}(k)$-2-submódulo irreductível de $\Lambda^{2}\left(U^{i}\right)$, para todo $i=10,12,19,20,25,26,27,28,54,60,101,258,259,263,298$. se segue que, $V_{3} \wedge V_{3} \simeq \mathfrak{s l}_{3}(k)$, o qual é absurdo. Da mesma forma tem-se que $V_{3}^{*}$ é um $\mathfrak{s l}_{3}(k)$-2-submódulo de $U^{i}$ e assim $V_{3}^{*} \wedge V_{3}^{*} \simeq V_{3}^{*}$ é um $\mathfrak{s l}_{3}(k)$-2-submódulo irreductível de $\Lambda^{2}\left(U^{i}\right)$, para todo $i=13,15,32,40,41,42,45,46,47,48,103,253,257,264,293$. se segue que, $V_{3}^{*} \wedge V_{3}^{*} \simeq \mathfrak{s l}_{3}(k)$, o qual também é absurdo. Por outro lado, $V_{0} \wedge V_{0}$ é um $\mathfrak{s l}_{3}(k)$-2-submódulo de $U^{i}$ para $i=31,53,65,67$, também $V_{0} \wedge V_{0}$ é $\mathfrak{s l}_{3}(k)$-módulo de $U^{i}$ para $i=37,57,140,146,178,180$ (ver tabelas: 3.34, 3.47, 3.56, 3.60, 3.63, 3.65 respectivamente.) e assim $V_{0} \wedge V_{0}$ é um $\mathfrak{s l}_{3}(k)$-2-submódulo irreductível de $\Lambda^{2}\left(U^{i}\right)$, logo $p \circ \varphi \circ \iota: \Lambda^{2}\left(V_{0} \curlywedge V_{0}\right) \rightarrow \mathfrak{s l}_{3}(k)$ é um $\mathfrak{s l}_{3}(k)$-isomorfismo. Uma contradição. Por ultimo, $V_{0}$ é um $\mathfrak{s l}_{3}(k)$-2-submódulo de $U^{i}$ e assim $V_{0} \wedge V_{0}=0$ é um $\mathfrak{s l}_{3}(k)$-2-submódulo de $\Lambda^{2}\left(U^{i}\right)$, para todo $i=39,59,139,143,144,145,148,149,179,184,254,260,292,294$, se segue que, $0=V_{0} \wedge V_{0} \simeq \mathfrak{s l}_{3}(k)$, o qual é absurdo.

Se continuamos argumentando da mesma forma como no caso $i=1$, procurando os submódulos tal como foi feito anteriormente em alguns casos de cima, logramos demonstrar (com fé, paciencia e tiempo) essa implicação, note que nenhum dessos $\mathfrak{s l}_{3}(k)$ módulos $\Lambda^{2}\left(U^{i}\right) \subseteq U^{i} \otimes U^{i}$ contém $\mathfrak{s l}_{3}(k)$-submódulos $A$ e $B$ irredutíveis de tal jeito que $A \otimes_{k} B \simeq\left(V_{3} \otimes_{k} V_{3}^{*}\right) / k \simeq \mathfrak{s l}_{3}(k)$. 


\section{Capítulo 4}

\section{As 2-álgebras de Lie simples de $\operatorname{dim}_{k}=17$ de posto toral 3}

Neste capítulo vamos estudar as 2-álgebras de Lie simples de dimensão 17 de posto toral 3, especialmente aquela da forma $(17: 3,0,2,2,2,2,2,2,2)$. Mostraremos que não existe uma 2-álgebra de Lie de tipo $(17: 3,0,2,2,2,2,2,2,2)$ contendo uma 2-subálgebra de Lie simples, e além disso, apresentaremos um método para tratar o caso quando esta álgebra contém uma 2-subálgebra de Lie não simples e não solúvel.

Em todo o capítulo, vamos supor que $(L,[2])$ é uma 2-álgebra de Lie simples de dimensão 17, de $M T(L)=3$, cuja decomposição de Cartan respeito a uma subálgebra de Cartan $H:=T \oplus N$ de $L$, é: $L=H \oplus \sum_{\xi \in G} \oplus L_{\xi}$, onde $G:=\langle\alpha, \beta, \gamma\rangle$ é um grupo abeliano elementar de ordem 8, $T$ é uma subálgebra toral de dimensão 3 , e $N$ é uma subálgebra de Lie 2-nilpotente.

Seguindo com o espírito da seção 2.4, temos os seguintes casos de 2-álgebras de Lie simples de dimensão 17 , de $M T(L)=3$ :
1. $(17: 3,7,1,1,1,1,1,1,1)$
10. $(17: 3,3,3,3,1,1,1,1,1)$
2. $(17: 3,6,2,1,1,1,1,1,1)$
11. $(17: 3,3,3,2,2,1,1,1,1)$
3. $(17: 3,5,3,1,1,1,1,1,1)$
12. $(17: 3,3,2,2,2,2,1,1,1)$
4. $(17: 3,5,2,2,1,1,1,1,1)$
13. $(17: 3,2,6,1,1,1,1,1,1)$
5. $(17: 3,4,4,1,1,1,1,1,1)$
14. $(17: 3,2,5,2,1,1,1,1,1)$
6. $(17: 3,4,3,2,1,1,1,1,1)$
15. $(17: 3,2,4,3,1,1,1,1,1)$
7. $(17: 3,4,2,2,2,1,1,1,1)$
16. $(17: 3,2,4,2,2,1,1,1,1)$
8. $(17: 3,3,5,1,1,1,1,1,1)$
17. $(17: 3,2,3,3,2,1,1,1,1)$
9. $(17: 3,3,4,2,1,1,1,1,1)$
18. $(17: 3,2,3,2,2,2,1,1,1)$ 


$\begin{array}{ll}\text { 19. }(17: 3,1,7,1,1,1,1,1,1) & \text { 31. }(17: 3,0,6,2,2,1,1,1,1) \\ \text { 20. }(17: 3,1,6,2,1,1,1,1,1) & \text { 32. }(17: 3,0,5,4,1,1,1,1,1) \\ \text { 21. }(17: 3,1,5,3,1,1,1,1,1) & \text { 33. }(17: 3,0,5,3,2,1,1,1,1) \\ \text { 22. }(17: 3,1,5,2,2,1,1,1,1) & \text { 34. }(17: 3,0,5,2,2,2,1,1,1) \\ \text { 23. }(17: 3,1,4,3,2,1,1,1,1) & \text { 35. }(17: 3,0,4,4,2,1,1,1,1) \\ \text { 24. }(17: 3,1,4,2,2,2,1,1,1) & \text { 36. }(17: 3,0,4,3,3,1,1,1,1) \\ \text { 25. }(17: 3,1,3,3,2,2,1,1,1) & \text { 37. }(17: 3,0,4,3,2,2,1,1,1) \\ \text { 26. }(17: 3,1,3,2,2,2,2,1,1) & \text { 38. }(17: 3,0,4,2,2,2,2,1,1) \\ \text { 27. }(17: 3,1,2,2,2,2,2,2,1) & \text { 39. }(17: 3,0,3,3,3,2,1,1,1) \\ \text { 28. }(17: 3,0,8,1,1,1,1,1,1) & \text { 40. }(17: 3,0,3,3,2,2,2,1,1) \\ \text { 29. }(17: 3,0,7,2,1,1,1,1,1) & \text { 41. }(17: 3,0,3,2,2,2,2,2,1) \\ \text { 30. }(17: 3,0,6,3,1,1,1,1,1) & \text { 42. }(17: 3,0,2,2,2,2,2,2,2) .\end{array}$

No caso 1., temos que $I:=N \oplus \sum_{\xi \in \Delta} \oplus L_{\xi}$ é um ideal de $L$. Uma contradição. Seja $e_{\gamma}$ um elemento da base de $L_{\gamma}$. Como $e_{\gamma}^{[2]} \in L_{\gamma}^{[2]} \subseteq H=T \oplus N$, então existem $t_{\gamma} \in T$ e $n_{\gamma} \in N$ tais que $e_{\gamma}^{[2]}=t_{\gamma}+n_{\gamma}$. Como $t_{\gamma} \in T$, existem $\delta_{1}, \delta_{2}, \delta_{3}$ em $k$, tais que $t_{\gamma}=\delta_{1} t_{1}+\delta_{2} t_{2}+\delta_{3} t_{3}$. Por outro lado, $0=\left[e_{\gamma}, e_{\gamma}^{[2]}\right]=\gamma\left(t_{\gamma}\right) e_{\gamma}+\left[e_{\gamma}, n_{\gamma}\right]$, $\operatorname{logo} a d\left(n_{\gamma}\right)\left(e_{\gamma}\right)=\gamma\left(t_{\gamma}\right) e_{\gamma}$. Como $n_{\gamma}$ é nilpotente, então $a d\left(n_{\gamma}\right)$ é nilpotente, portanto $\gamma\left(t_{\gamma}\right)=\delta_{3}=0$. Assim, $e_{\gamma}^{[2]}=\delta_{1} t_{1}+\delta_{2} t_{2}+n_{\gamma}$. Escolhendo $\delta_{1} \neq 0$ temos que $t_{\gamma} \neq 0$ e $\alpha\left(t_{\gamma}\right)=\delta_{1} \neq 0$, logo, pela proposição 2.4, $L_{\alpha} \simeq L_{\alpha+\gamma}$. Portanto os casos 2. até 41 . exceto 27. são impossíveis, já que $\operatorname{dim}_{k}\left(L_{\alpha}\right) \neq \operatorname{dim}_{k}\left(L_{\alpha+\gamma}\right)$. Para o caso 27. temos o seguinte: escolhendo $\delta_{1} \neq \delta_{2}$, temos que

$(\alpha+\beta)\left(t_{\gamma}\right)=(\alpha+\beta)\left(\delta_{1} t_{1}+\delta_{2} t_{2}\right)=\delta_{1}+\delta_{2} \neq 0$, logo pela proposição 2.4. temos que $L_{\alpha+\gamma} \simeq L_{\alpha+\beta+\gamma}$, mas $2=\operatorname{dim}_{k}\left(L_{\alpha+\gamma}\right) \neq \operatorname{dim}_{k}\left(L_{\alpha+\beta+\gamma}\right)=1$, logo o caso 27 . não é possível. Portanto,

Proposição 4.1. Não existem 2-álgebras de Lie $(L,[2])$, simples de dimensão 17 de posto toral 3, com decomposição de Cartan respeito a $T$ dada por: $L:=T \oplus \sum_{\xi \in G} \oplus L_{\xi}$, onde $G:=\langle\alpha, \beta, \gamma\rangle$ é um grupo abeliano elementar de ordem 8 e $\operatorname{dim}_{k}\left(L_{\xi}\right) \neq 2$ para algum $\xi \in G$.

A álgebra de Lie do item 42. é abordada na seção seguinte. 


\section{1 $L=T \oplus \sum_{\xi \in G} \oplus L_{\xi}$, onde $\operatorname{dim}_{k}\left(L_{\xi}\right)=2, \forall \xi \in G$.}

Aqui propomos uma maneira de abordar o estudo de $(17: 3,0,2,2,2,2,2,2,2)$ via teoria de representação de álgebras.

Pode acontecer que $(L,[2])$ contenha uma 2-subálgebra de Lie :

a. Simples

b. Não simples e não solúvel

c. Solúvel

Agora, estudemos $(L,[2])$ da forma (17:3, 0, 2, 2, 2, 2, 2, 2, 2) quando uma 2-subálgebra de Lie de tipo $a ., b$., ou $c$. fique mergulhada nela. Primeiro estudemos o caso quando $(L,[2])$ contém uma 2-subálgebra de Lie simples.

\subsection{1 (L, [2]) contém uma 2-subálgebra isomorfa a $\left(\mathfrak{s l}_{3}(k), 2\right)$}

Vai ser apresentada aqui uma construção de $(L,[2])$ baseada na representação de $\mathfrak{s l}_{3}(k)$ em 2-módulos de dimensão 8 tal como foi feita na seção 3.3. A idéia dessa construção vem da observação de que $\left(\mathfrak{s l}_{3}(k), 2\right)$ aparece como 2 -subálgebra de $(L,[2])$. De fato, considere á subálgebra de Lie de $L$

$$
S_{\alpha, \beta}:=\operatorname{span}\left\{t_{1}, t_{2}, a_{1}^{\alpha}, a_{2}^{\alpha}, b_{1}^{\beta}, b_{2}^{\beta}, c_{1}^{\alpha+\beta}, c_{2}^{\alpha+\beta}\right\}
$$

com multilicação de Lie dada na tabela seguinte

\begin{tabular}{|c||c|c|c|c|c|c|c|c|}
\hline & $t_{1}$ & $t_{2}$ & $a_{1}^{\alpha}$ & $a_{2}^{\alpha}$ & $b_{1}^{\beta}$ & $b_{2}^{\beta}$ & $c_{1}^{\alpha+\beta}$ & $c_{2}^{\alpha+\beta}$ \\
\hline \hline$t_{1}$ & $t_{1}$ & 0 & $a_{1}^{\alpha}$ & $a_{2}^{\alpha}$ & 0 & 0 & $c_{1}^{\alpha+\beta}$ & $c_{2}^{\alpha+\beta}$ \\
\hline$t_{2}$ & 0 & $t_{2}$ & 0 & 0 & $b_{1}^{\beta}$ & $b_{2}^{\beta}$ & $c_{1}^{\alpha+\beta}$ & $c_{2}^{\alpha+\beta}$ \\
\hline$a_{1}^{\alpha}$ & $a_{1}^{\alpha}$ & 0 & 0 & $t_{2}$ & $c_{1}^{\alpha+\beta}$ & 0 & 0 & $b_{2}^{\beta}$ \\
\hline$a_{2}^{\alpha}$ & $a_{2}^{\alpha}$ & 0 & $t_{2}$ & 0 & 0 & $c_{2}^{\alpha+\beta}$ & $b_{1}^{\beta}$ & 0 \\
\hline$b_{1}^{\beta}$ & 0 & $b_{1}^{\beta}$ & $c_{1}^{\alpha+\beta}$ & 0 & 0 & $t_{1}$ & 0 & $a_{2}^{\alpha}$ \\
\hline$b_{2}^{\beta}$ & 0 & $b_{2}^{\beta}$ & 0 & $c_{2}^{\alpha+\beta}$ & $t_{1}$ & 0 & $a_{1}^{\alpha}$ & 0 \\
\hline$c_{1}^{\alpha+\beta}$ & $c_{1}^{\alpha+\beta}$ & $c_{1}^{\alpha+\beta}$ & 0 & $b_{1}^{\beta}$ & 0 & $a_{1}^{\alpha}$ & 0 & $t_{1}+t_{2}$ \\
\hline$c_{2}^{\alpha+\beta}$ & $c_{2}^{\alpha+\beta}$ & $c_{2}^{\alpha+\beta}$ & $b_{2}^{\beta}$ & 0 & $a_{2}^{\alpha}$ & 0 & $t_{1}+t_{2}$ & 0 \\
\hline
\end{tabular}

Tabela 4.1: A 2-álgebra de Lie $S_{\alpha, \beta}$

onde os elementos da diagonal são resultados da 2-aplicação nas elementos de suas linhas ou colunas correspondentes. 
$S_{\alpha, \beta}$ é uma 2-subálgebra de Lie simples de $(L,[2])$ de dimensão 8 , que é isomorfa a $\left(\mathfrak{s l}_{3}(k), 2\right)$, por meio da seguinte identificação:

$$
\begin{aligned}
S_{\alpha, \beta} & \stackrel{\phi}{ } \mathfrak{s l}_{3}(k) \\
t_{1} & \mapsto h_{2} \\
t_{2} & \mapsto h_{1} \\
a_{1}^{\alpha} & \mapsto e_{12} \\
a_{2}^{\alpha} & \mapsto e_{21} \\
b_{1}^{\beta} & \mapsto e_{23} \\
b_{2}^{\beta} & \mapsto e_{32} \\
c_{1}^{\alpha+\beta} & \mapsto e_{13} \\
c_{2}^{\alpha+\beta} & \mapsto e_{31}
\end{aligned}
$$

Dessa forma, $\left(\mathfrak{s l}_{3}(k), 2\right)$ se representa na álgebra $(L,[2])$ vía a representação adjunta. É natural, portanto, procurar a realização de $(L,[2])$ em espaços de representação de dimensão 17 de $\left(\mathfrak{s l}_{3}(k), 2\right)$. Isso é feito da seguinte maneira: a álgebra $\left(\mathfrak{s l}_{3}(k), 2\right)$ se representa em um $k$-espaço vetorial de dimensão 8 , onde os elementos da base destes espaços tem pesos $(0,0),(1,0),(0,1),(1,1)$ (cada um com multiplicidade 2), mas isso já foi feito na seção 3.3. Logo a álgebra $(L,[2])$ é construída sobre o $k$-espaço vetorial

$$
G\left(U^{i}\right):=\mathfrak{s l}_{3}(k) \oplus k t_{3} \oplus U^{i} \text { para todo } 1 \leq i \leq 365
$$

onde $U^{i}$ são os $\mathfrak{s l}_{3}(k)$-2-módulos de dimensão 8 que foram achados e enumerados na seção 3.3

\subsubsection{1. $\left(G\left(U^{i}\right),[],\right)$ é uma k-álgebra}

Para que $G\left(U^{i}\right)$ tenha estrutura de $k$-álgebra, precisamos definir uma aplicação $k$-bilinear

$$
\text { [,] : } G\left(U^{i}\right) \times G\left(U^{i}\right) \rightarrow G\left(U^{i}\right) .
$$

Essa aplicação $k$-bilinear será definida em pares de elementos nos diferentes subespaços que compõem $G\left(U^{i}\right)$. Definir esta aplicação entre os elementos de $\mathfrak{s l}_{3}(k)$ com $\mathfrak{s l}_{3}(k)$ ou, $\mathfrak{s l}_{3}(k)$ com $k t_{3}$ ou, $\mathfrak{s l}_{3}(k)$ com $U^{i}$ ou, $k t_{3}$ com $U^{i}$ pode ser feito como segue:

$A_{1}$. O colchete entre dois elementos de $\mathfrak{s l}_{3}(k)$ é o usual, isto é: $\left[e_{i j}, e_{k l}\right]:=\delta_{j k} e_{i l}-\delta_{l i} e_{k j}$ para todo $e_{i j} \in \mathfrak{s l}_{3}(k)$.

$A_{2}$. Se $A \in \mathfrak{s l}_{3}(k)$ e $u \in U^{i}$, então $[A, u]:=u$. A, isto é, o colchete é dado pela 2-representação de $\mathfrak{s l}_{3}(k)$ em $U^{i}$.

$A_{3}$. Como $A n n_{L}\left(t_{3}\right)=\mathfrak{s l}_{3}(k) \oplus k t_{3}$, então $\left[A, t_{3}\right]:=0$ para todo $A \in \mathfrak{s l}_{3}(k)$.

$A_{4}$. Como $\gamma\left(t_{3}\right)=1$ temos: $\left[u, t_{3}\right]:=u$ para todo $u \in U^{i}$.

O teorema 3.2 permite definir uma aplicação $k$-bilinear entre elementos de

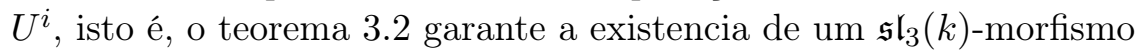
$\Lambda^{2}\left(U^{i}\right) \stackrel{\varphi}{\rightarrow} \mathfrak{s l}_{3}(k) \oplus k t_{3}$, o qual nos permite definir o colchete entre dois elementos de $U^{i}$ assim: 
$A_{5}$. Se existe um $\mathfrak{s l}_{3}(k)$-morfismo $\varphi: \Lambda^{2}\left(U^{i}\right) \rightarrow \mathfrak{s l}_{3}(k) \oplus k t_{3}$, definimos o colchete entre dois elementos de $U^{i}$ como: $[u, v]:=\varphi(u \wedge v)$ para todo $u, v \in U^{i}$.

Em resumo, as expressões $A_{1}, A_{2}, A_{3}, A_{4}$ e $A_{5}$ definen aplicações $k$-bilineares a valores em $\left(G\left(U^{i}\right),[],\right)$ e definidas em $U^{i} \times U^{i}, \mathfrak{s l}_{3}(k) \times \mathfrak{s l}_{3}(k)$, etc. Essas aplicações se estendem por bilinearidade a uma aplicação $k$-bilinear alternada

[,] : $G\left(U^{i}\right) \times G\left(U^{i}\right) \rightarrow G\left(U^{i}\right)$. Portanto, se $\left(G\left(U^{i}\right),[],\right)$ satisfaz os criterios $A_{1}$, $A_{2}, A_{3}, A_{4}, A_{5}$, então $\left(G\left(U^{i}\right),[],\right):=\left(\mathfrak{s l}_{3}(k) \oplus k t_{3} \oplus U^{i},[],\right)$ é uma $k$-álgebra de dimensão 17 .

4.1.1.2. Existencia de um $\mathfrak{s l}_{3}(k)$-morfismo $\varphi: \Lambda^{2}\left(U^{i}\right) \rightarrow \mathfrak{s l}_{3}(k) \oplus k t_{3}$.

$\mathrm{Na}$ seção 3.3 , temos uma lista de $365 \mathfrak{s l}_{3}(k)$-2-módulos $U^{i}$ de dimensão 8 . Nesta seção procuramos os módulos $U^{i}$ dessa lista que cumprem as condições a. e b. do teorema 3.2 (e portanto $A_{5}$ ). Estamos interessados em aqueles $\mathfrak{s l}_{3}(k)$-2-módulos $U^{i}$ que cindem como é sugerido pelo teorema 3.2.

Os $\mathfrak{s l}_{3}(k)$-2-módulos $U^{i}$, para $i=1,3,6,8,9,11,14,16,17,18,21,22,23,24$, $29,30,33,34,35,36,43,44,49,50,51,52,55,56,61,62,63,64,66,68,69$ até $100,102,104$ até $137,140,141,146,147,150$ até 177, 181 até 252, 255, 256, 261, 262, 265 até 291, 295 até 297, 299 até 364 e, 365 não satisfazem a condições a. e b. do teorema 3.2. Já que, pelo geral eles não cindem, pois dependem de algumos parametros que pertencem a $k$; se eles cindem (isto somente acontece se todos os parametros são nulos) vamos ter que eles podem satisfazer a condição a. ou a condição b. do teorema 3.2, mas não cumprem simultaneamente estas condições. Por exemplo, da tabela 3.79 tem-se que o $\mathfrak{s l}_{3}(k)$-2-módulo $U^{1}:=$ $\left(V_{3}^{*} \curlywedge V_{0}\right) \curlywedge\left(V_{3}^{*} \curlywedge V_{0}\right)$ cinde se, e só se, $\delta_{i}=0$. Além do mais, se $P_{3}:=V_{3}^{*} \curlywedge V_{0}$, $P_{4}:=V_{0}$ e $P:=V_{3}^{*}<V_{0}$, então $P_{4} \subseteq P_{3}, P_{3} / P_{4} \simeq V_{3}^{*}$, e $P \oplus P_{3}=U^{1}$. Portanto $U^{1}$ cumpre a condição b. do teorema 3.2 , mas não satisfaz a condição a. Assim, não é possivel achar um $\mathfrak{s l}_{3}(k)$-2-morfismo $\varphi: \Lambda^{2}\left(U^{i}\right) \rightarrow \mathfrak{s l}_{3}(k) \oplus k t_{3}$.

Agora, estudemos os $\mathfrak{s l}_{3}(k)$-2-módulos restante da lista.

1. Seja $U^{2}:=\left(V_{3} \curlywedge V_{0}\right) \curlywedge\left(V_{3}^{*} \curlywedge V_{0}\right)$. Como vimos na seção 3.3.1.1:

$$
U^{2} \simeq\left(V_{3} \curlywedge V_{0}\right) \oplus\left(V_{3}^{*} \curlywedge V_{0}\right) \Longleftrightarrow \mu_{i}=0
$$

Se $U^{2} \simeq\left(V_{3} \wedge V_{0}\right) \oplus\left(V_{3}^{*} \wedge V_{0}\right)$, então $U^{2}$ cumpre a. e b. do teorema 3.2. De fato, se $Q_{1}:=V_{3} \wedge V_{0}, Q_{2}:=V_{0}, P_{3}:=V_{3}^{*} \wedge V_{0}, P_{4}:=V_{0}, Q:=V_{3}^{*} \curlywedge V_{0}$ e $P:=V_{3}<V_{0}$ temos que:

a) $Q_{2} \subseteq Q_{1}, Q_{1} / Q_{2} \simeq V_{3}$ e, $Q_{1} \oplus Q=U^{2}$.

b) $P_{4} \subseteq P_{3}, P_{3} / P_{4} \simeq V_{3}^{*}$ e, $P_{3} \oplus P=U^{2}$.

Assim, se $U^{2} \simeq\left(V_{3} \wedge V_{0}\right) \oplus\left(V_{3}^{*} \wedge V_{0}\right)$, então pelo teorema 3.2 existe um $\mathfrak{s l}_{3}(k)$-morfismo $\varphi: \Lambda^{2}\left(U^{2}\right) \rightarrow \mathfrak{s l}_{3}(k) \oplus k t_{3}$.

Pela seção 3.3.1.3 temos que: $U^{5} \simeq\left(V_{3} \wedge V_{0}\right) \oplus\left(V_{3}^{*} \wedge V_{0}\right) \Longleftrightarrow \delta_{i}=0$. Portanto, argumentando da mesma forma como acima também temos que existe um $\mathfrak{s l}_{3}(k)$-morfismo $\varphi: \Lambda^{2}\left(U^{5}\right) \rightarrow \mathfrak{s l}_{3}(k) \oplus k t_{3}$. 
2. Seja $U^{4}:=\left(V_{0} \wedge V_{3}^{*}\right) \curlywedge\left(V_{3} \wedge V_{0}\right)$. Temos que $U^{4}$ satisfaz a. e b. do teorema 3.2, se $U^{4} \simeq\left(V_{0} \oplus V_{3}^{*}\right) \oplus\left(V_{3} \wedge V_{0}\right) \Longleftrightarrow \delta_{i}=\delta=\alpha=\zeta=0$ (ver seção 3.3.1.2). De fato, se $Q_{1}:=V_{3} \wedge V_{0}, Q_{2}:=V_{0}, P_{3}:=V_{3}^{*}, P_{4}:=0, Q:=V_{0} \oplus V_{3}^{*}$ e, $P:=\left(V_{3} \wedge V_{0}\right) \oplus V_{0}$ temos :

a) $Q_{2} \subseteq Q_{1}, Q_{1} / Q_{2} \simeq V_{3}$ e, $Q_{1} \oplus Q=U^{4}$.

b) $P_{4} \subseteq P_{3}, P_{3} / P_{4} \simeq V_{3}^{*}$ e, $P_{3} \oplus P=U^{4}$.

Portanto, existe um $\mathfrak{s l}_{3}(k)$-morfismo $\varphi: \Lambda^{2}\left(U^{4}\right) \rightarrow \mathfrak{s l}_{3}(k) \oplus k t_{3}$.

Analogamente, acontece com os $\mathfrak{s l}_{3}(k)-2$ - módulos: $U^{13}, U^{45}, U^{46}, U^{57}, U^{58}$, $U^{138}, U^{139}, U^{253}$ e $U^{254}$ (ver seções: 3.3.1.7., 3.3.1.23., 3.3.1.24., 3.3.1.29., 3.3.1.30., 3.3.1.37., 3.3.1.38., 3.3.1.49. e, 3.3.1.50.).

3. Seja $U^{7}:=\left(V_{0} \wedge V_{3}\right) \curlywedge\left(V_{3}^{*} \curlywedge V_{0}\right)$. Pela seção 3.3.1.4 temos que

$$
U^{7} \simeq\left(V_{0} \oplus V_{3}\right) \oplus\left(V_{3}^{*} \curlywedge V_{0}\right) \Longleftrightarrow \delta_{i}=\omega=\sigma=\theta=0
$$

além disso, $U^{7}$ cumpre a. e b. do teorema 3.2 se, e somente se, $U^{7} \simeq\left(V_{0} \oplus V_{3}\right) \oplus\left(V_{3}^{*} \wedge V_{0}\right)$. De fato, se $Q_{1}:=V_{3}, Q_{2}:=0, P_{3}:=V_{3}^{*} \wedge V_{0}$, $P_{4}:=V_{0}, Q:=\left(V_{3}^{*} \wedge V_{0}\right) \oplus V_{0}$ e, $P:=V_{0} \oplus V_{3}$, então

a) $Q_{2} \subseteq Q_{1}, Q_{1} / Q_{2} \simeq V_{3}$ e $Q_{1} \oplus Q=U^{7}$.

b) $P_{4} \subseteq P_{3}, P_{3} / P_{4} \simeq V_{3}^{*}$ e $P_{3} \oplus P=U^{7}$.

Então pelo teorema 3.2 existe um $\mathfrak{s l}_{3}(k)$-morfismo $\varphi: \Lambda^{2}\left(U^{7}\right) \rightarrow \mathfrak{s l}_{3}(k) \oplus k t_{3}$. Da mesma forma tem-se para os módulos: $U^{10}, U^{25}, U^{26}, U^{37}, U^{38}, U^{144}$, $U^{145}, U^{259}, U^{260}$ (ver seções: 3.3.1.5., 3.3.1.11., 3.3.1.12., 3.3.1.17., 3.3.1.18., 3.3.1.41, 3.3.1.42., 3.3.1.42., 3.3.1.53. e, 3.3.1.54.).

4. Seja $U^{12}:=\left(V_{0} \wedge V_{3}^{*}\right) \curlywedge\left(V_{0} \wedge V_{3}\right)$. Pela seção 3.3.1.6 vemos que

$$
U^{12} \simeq\left(V_{0} \oplus V_{3}^{*}\right) \oplus\left(V_{0} \oplus V_{3}\right) \Longleftrightarrow \delta_{i}=\omega=\theta=\sigma=\delta=\alpha=\zeta=0
$$

e se $U^{12} \simeq\left(V_{0} \oplus V_{3}^{*}\right) \oplus\left(V_{0} \oplus V_{3}\right)$, então temos a. e b. do teorema 3.2. De fato, se $Q_{1}:=V_{3}, Q_{2}:=0, P_{3}:=V_{3}^{*}, P_{4}:=0, Q:=\left(V_{0} \oplus V_{3}^{*}\right) \oplus V_{0} \mathrm{e}$ $P:=\left(V_{0} \oplus V_{3}\right) \oplus V_{0}$ então

a) $Q_{2} \subseteq Q_{1}, Q_{1} / Q_{2} \simeq V_{3}$ e, $Q_{1} \oplus Q=U^{12}$.

b) $P_{4} \subseteq P_{3}, P_{3} / P_{4} \simeq V_{3}^{*}$ e, $P_{3} \oplus P=U^{12}$.

Pelo teorema 3.2 existe um $\mathfrak{s l}_{3}(k)$-morfismo $\varphi: \Lambda^{2}\left(U^{12}\right) \rightarrow \mathfrak{s l}_{3}(k) \oplus k t_{3}$. Da mesma forma, também tem-se para os módulos: $U^{15}, U^{20}, U^{27}, U^{28}, U^{32}$, $U^{39}, U^{40}, U^{42}, U^{47}, U^{48}, U^{54}, U^{59}, U^{60}, U^{65}, U^{67}, U^{101}, U^{103}, U^{142}, U^{143}$, $U^{148}, U^{149}, U^{178}, U^{179}, U^{180}, U^{184}, U^{257}, U^{258}, U^{263}, U^{264}, U^{292}, U^{293}$, $U^{294}, U^{298}$ (ver seções: 3.3.1.8, 3.3.1.10,3.3.1.13,3.3.1.14,3.3.1.16, 3.3.1.19, 3.3.1.20., 3.3.1.22., 3.3.1.25., 3.3.1.26., 3.3.1.28., 3.3.1.31., 3.3.1.32., 3.3.1.33., 3.3.1.34., 3.3.1.35., 3.3.1.36., 3.3.1.39., 3.3.1.40., 3.3.1.43., 3.3.1.44., 3.3.1.45., 3.3.1.46., 3.3.1.47., 3.3.1.48., 3.3.1.51., 3.3.1.52., 3.3.1.55., 3.3.1.56., 3.3.1.57., 3.3.1.58., 3.3.1.59., 3.3.1.60.). 
5. Seja $U^{19}:=\left[V_{3}^{*} \curlywedge\left(V_{0} \curlywedge V_{0}\right)\right] \curlywedge V_{3}$. Pela seção 3.3.1.9. temos que

$$
U^{19} \simeq\left[V_{3}^{*} \curlywedge\left(V_{0}<V_{0}\right)\right] \oplus V_{3} \Longleftrightarrow \delta_{i}=0 .
$$

Além disso, se $U^{19} \simeq\left[V_{3}^{*} \curlywedge\left(V_{0} \wedge V_{0}\right)\right] \oplus V_{3}$, então temos a. e b. do teorema 3.2. De fato, se $Q_{1}:=V_{3}, Q_{2}:=0, P_{3}:=V_{3}^{*} \wedge\left(V_{0} \wedge V_{0}\right), P_{4}:=V_{0} \wedge V_{0}$, $Q:=V_{3}^{*} \curlywedge\left(V_{0} \wedge V_{0}\right)$ e $P:=V_{3}$, então

a) $Q_{2} \subseteq Q_{1}, Q_{1} / Q_{2} \simeq V_{3}$ e, $Q_{1} \oplus Q=U^{19}$.

b) $P_{4} \subseteq P_{3}, P_{3} / P_{4} \simeq V_{3}^{*}$ e, $P_{3} \oplus P=U^{19}$.

Pelo teorema 3.2 existe um $\mathfrak{s l}_{3}(k)$-morfismo $\varphi: \Lambda^{2}\left(U^{19}\right) \rightarrow \mathfrak{s l}_{3}(k) \oplus k t_{3}$. Da mesma forma, isto tem-se para o $\mathfrak{s l}_{3}(k)$-módulo $U^{31}$ (ver seção 3.3.1.15).

6. Seja $U^{41}:=\left[V_{3} \curlywedge\left(V_{0} \curlywedge V_{0}\right)\right] \curlywedge V_{3}^{*}$. Pela seção 3.3.1.21 temos que

$$
U^{41} \simeq\left[V_{3} \curlywedge\left(V_{0} \wedge V_{0}\right)\right] \oplus V_{3}^{*} \Longleftrightarrow \delta_{i}=0 .
$$

Além disso, Se $U^{41} \simeq\left[V_{3} \curlywedge\left(V_{0}<V_{0}\right)\right] \oplus V_{3}^{*}$ então temos a. e b. do teorema 3.2. De fato, se $Q_{1}:=V_{3} \curlywedge\left(V_{0} \curlywedge V_{0}\right), Q_{2}:=V_{0} \curlywedge V_{0}, P_{3}:=V_{3}^{*}, P_{4}:=0$, $Q:=V_{3}^{*}$ e $P:=V_{3} \wedge\left(V_{0} \wedge V_{0}\right)$, então

a) $Q_{2} \subseteq Q_{1}, Q_{1} / Q_{2} \simeq V_{3}$ e, $Q_{1} \oplus Q=U^{41}$.

b) $P_{4} \subseteq P_{3}, P_{3} / P_{4} \simeq V_{3}^{*}$ e, $P_{3} \oplus P=U^{41}$.

Pelo teorema 3.2 existe um $\mathfrak{s l}_{3}(k)$-morfismo $\varphi: \Lambda^{2}\left(U^{41}\right) \rightarrow \mathfrak{s l}_{3}(k) \oplus k t_{3}$. Da mesma forma, também tem-se para o $\mathfrak{s l}_{3}(k)$-2-módulo $U^{53}$ (ver seção 3.3.1.27).

Em resumo, temos que os seguintes $\mathfrak{s l}_{3}(k)$-2-módulos $U$ da forma:

a) $U^{a}:=\left(V_{0} \oplus V_{3}^{*}\right) \oplus\left(V_{0} \oplus V_{3}\right)$

b) $U^{b}:=\left(V_{0} \oplus V_{3}^{*}\right) \oplus\left(V_{3} \wedge V_{0}\right)$

c) $U^{c}:=\left(V_{0} \oplus V_{3}\right) \oplus\left(V_{3}^{*} \wedge V_{0}\right)$

d) $U^{d}:=\left(V_{3}^{*} \wedge V_{0}\right) \oplus\left(V_{3} \wedge V_{0}\right)$

e) $U^{e}:=V_{3}^{*} \oplus\left[V_{3} \wedge\left(V_{0} \wedge V_{0}\right)\right]$

f) $U^{f}:=V_{3} \oplus\left[V_{3}^{*} \wedge\left(V_{0} \wedge V_{0}\right)\right]$

cumprem a condição 1 . do teorema 3.2., portanto existe um $\mathfrak{s l}_{3}(k)$-morfismo

$$
\varphi: \Lambda^{2}\left(U^{j}\right) \rightarrow \mathfrak{s l}_{3}(k) \oplus k t_{3}
$$

para todo $j=a, b, c, d, e, f$.

Assim que, das condições $A_{1}, A_{2}, A_{3}, A_{4}$ e $A_{5}$ temos que $\left(G\left(U^{j}\right),[],\right)$ é uma $k$-álgebra de dimensão 17 , para todo $j=a, b, c, d, e, f$. 
4.1.1.3. $\left(G\left(U^{j}\right),[],\right) k$-álgebra de Lie.

Temos que o par $\left(G\left(U^{j}\right),[],\right)$, para $j=a, b, c, d, e, f$ é uma $k$-álgebra, além disso [,] é uma aplicação $k$-bilinear alternada. Assim, para ver que $\left(G\left(U^{j}\right),[],\right)$ (para $j=a, b, c, d, e, f)$ é uma álgebra de Lie sobre $k$ é necessario mostrar a identidade de Jacobi. Isso é feito considerando ternas de elementos nos diferentes espaços $\mathfrak{s l}_{3}(k), k t_{3}, U^{j}$. A identidade de Jacobi será tomada na forma

$$
0=J(a, b, c):=[a,[b, c]]+[b,[a, c]]+[c,[a, b]] .
$$

Agora, para cada $\mathfrak{s l}_{3}(k)$-2-módulos $U^{j}, \operatorname{com} j=a, b, c, d, e, f$, vejamos se $\left(G\left(U^{j}\right),[],\right)$ tem estrutura de $k$-álgebra de Lie, verificando a identidade de Jacobi.

a) Temos que

$$
G\left(U^{a}\right)=\mathfrak{s l}_{3}(k) \oplus k t_{3} \oplus U^{a}
$$

é uma $k$-álgebra. Precisamos saber se ela é uma $k$-álgebra de Lie, para isso, primeiro definamos explicitamente o $\mathfrak{s l}_{3}(k)$-morfismo $\varphi: \Lambda^{2}\left(U^{a}\right) \rightarrow \mathfrak{s l}_{3}(k) \oplus k t_{3}$, e logo verificamos a identidade de Jacobi. De fato, seja $U^{a}:=\left\langle v, v_{1}, v_{2}, v_{3}, k, k_{1}, k_{2}, k_{3}\right\rangle$ (ver tabela 3.22).

Como $U^{a}:=\left(V_{0} \oplus V_{3}^{*}\right) \oplus\left(V_{0} \oplus V_{3}\right)$ então pelo exemplo 1.21.5 :

$$
\begin{aligned}
\Lambda^{2}\left(U^{a}\right) \simeq & \Lambda^{2}\left(V_{0} \oplus V_{3}^{*}\right) \oplus\left(\left(V_{0} \oplus V_{3}^{*}\right) \otimes\left(V_{0} \oplus V_{3}\right)\right) \oplus \Lambda^{2}\left(V_{0} \oplus V_{3}\right) \\
\simeq & \left(V_{0} \wedge V_{0}\right) \oplus\left(V_{0} \otimes_{k} V_{3}^{*}\right) \oplus\left(V_{3}^{*} \wedge V_{3}^{*}\right) \oplus\left(V_{0} \otimes_{k} V_{0}\right) \oplus\left(V_{0} \otimes_{k} V_{3}\right) \\
& \oplus\left(V_{3}^{*} \otimes_{k} V_{0}\right) \oplus\left(V_{3}^{*} \otimes_{k} V_{3}\right) \oplus\left(V_{0} \wedge V_{0}\right) \oplus\left(V_{0} \otimes_{k} V_{3}\right) \oplus\left(V_{3} \wedge V_{3}\right) \\
\simeq & \left(V_{0} \otimes_{k} V_{3}^{*}\right) \oplus\left(V_{3}^{*} \wedge V_{3}^{*}\right) \oplus\left(V_{0} \otimes_{k} V_{0}\right) \oplus\left(V_{0} \otimes_{k} V_{3}\right) \\
& \oplus\left(V_{3}^{*} \otimes_{k} V_{0}\right) \oplus\left(V_{3}^{*} \otimes_{k} V_{3}\right) \oplus\left(V_{0} \otimes_{k} V_{3}\right) \oplus\left(V_{3} \wedge V_{3}\right) .
\end{aligned}
$$

Logo temos os seguintes $\mathfrak{s l}_{3}(k)$-submódulos de $U^{a} \wedge U^{a}$ :

- $U_{1}^{a}:=\left(V_{0} \otimes_{k} V_{3}\right) \simeq\left(V_{0} \wedge V_{3}\right)=\left\langle v \wedge v_{1}, v \wedge v_{2}, v \wedge v_{3}\right\rangle \simeq V_{3}$.

- $U_{2}^{a}:=\left(V_{3} \otimes_{k} V_{0}\right) \simeq\left(V_{3} \wedge V_{0}\right)=\left\langle v_{1} \wedge k, v_{2} \wedge k, v_{3} \wedge k\right\rangle \simeq V_{3}$.

- $U_{3}^{a}:=\left(V_{3}^{*} \otimes_{k} V_{0}\right) \simeq\left(V_{3}^{*} \wedge V_{0}\right)=\left\langle v \wedge k_{1}, v \wedge k_{2}, v \wedge k_{3}\right\rangle \simeq V_{3}^{*}$.

- $U_{4}^{a}:=\left(V_{0} \otimes_{k} V_{3}^{*}\right) \simeq\left(V_{0} \wedge V_{3}^{*}\right)=\left\langle k \wedge k_{1}, k \wedge k_{2}, k \wedge k_{3}\right\rangle \simeq V_{3}^{*}$.

- $U_{5}^{a}:=\left(V_{3} \wedge V_{3}\right)=\left\langle v_{1} \wedge v_{2}, v_{1} \wedge v_{3}, v_{2} \wedge v_{3}\right\rangle \simeq V_{3}$.

- $U_{6}^{a}:=\left(V_{3}^{*} \wedge V_{3}^{*}\right)=\left\langle k_{1} \wedge k_{2}, k_{1} \wedge k_{3}, k_{2} \wedge k_{3}\right\rangle \simeq V_{3}^{*}$.

- $U_{7}^{a}:=\left(V_{3} \otimes_{k} V_{3}^{*}\right) \simeq\left(V_{3} \wedge V_{3}^{*}\right)=\left\langle v_{1} \wedge k_{j}, v_{2} \wedge k_{j}, v_{3} \wedge k_{j}: j=1,2,3\right\rangle$.

- $U_{8}^{a}:=V_{0} \otimes_{k} V_{0} \simeq V_{0} \wedge V_{0}=\langle v \wedge k\rangle$

Vejamos como está defenida $\varphi$ em cada um destos submódulos, isto é, ache$\left.\operatorname{mos} \varphi\right|_{U_{i}^{a}}$. Então temos que $\varphi\left(U_{i}^{a}\right)$ é um $\mathfrak{s l}_{3}(k)$-submódulos de $\mathfrak{s l}_{3}(k) \oplus k t_{3}$, para cada $i=1,2,3,4,5,6,7,8$ assim:

$$
U_{i}^{a} \rightarrow k t_{3}, \text { ou } U_{i}^{a} \rightarrow 0 \text {, ou } U_{i}^{a} \rightarrow \mathfrak{s l}_{3}(k) .
$$

Suponhamos que $U_{i}^{a} \rightarrow k t_{3}\left(U_{i}^{a} \rightarrow \mathfrak{s l}_{3}(k)\right)$ para $i=1,2,3,4,5,6$, 8. Como $V_{3}, V_{3}^{*}, k t_{3}$ são $\mathfrak{s l}_{3}(k)$-módulos irredutíveis, então pelo lema de Schur (lema 
1.19) temos que $U_{i}^{a} \simeq k t_{3}\left(U_{i}^{a} \simeq \mathfrak{s l}_{3}(k)\right)$ para todo $i=1,2, \ldots, 6,8$. Uma contradição. Portanto $\left.\varphi\right|_{U_{i}^{a}}=0$, para $i=1, \ldots, 6,8$.

Graças ao exemplo 3.1 temos que $\left.\varphi\right|_{U_{7}^{a}}=g$. Assim, $\varphi$ é definida como:

$$
\begin{array}{rll}
\Lambda^{2}\left(U^{a}\right) & \stackrel{\varphi}{\longrightarrow} \mathfrak{s l}_{3}(k) \oplus k t_{3} \\
v \wedge v_{i} & \mapsto \quad 0 \quad i=1,2,3 . \\
v \wedge k_{i} & \mapsto \quad 0 \quad i=1,2,3 . \\
v_{i} \wedge k & \mapsto \quad 0 \quad i=1,2,3 . \\
k \wedge k_{i} & \mapsto \quad 0 \quad i=1,2,3 . \\
v_{i} \wedge v_{k} & \mapsto \quad 0 \quad 1 \leq i<j \leq 3 . \\
k_{i} \wedge k_{j} & \mapsto \quad 0 \quad 1 \leq i<j \leq 3 . \\
v \wedge k & \mapsto 0 \\
\left.\varphi\right|_{U_{7}^{a}} & =g
\end{array}
$$

Agora, pela tabela 3.22 temos:

$J\left(v_{1}, k_{1}, k_{2}\right)=\left[v_{1},\left[k_{1}, k_{2}\right]\right]+\left[k_{1},\left[v_{1}, k_{2}\right]\right]+\left[k_{2},\left[v_{1}, k_{1}\right]\right]=$ $=\left[k_{1}, e_{21}\right]+\left[k_{2}, h_{2}+t_{3}\right]=k_{2}+k_{2}+k_{2}=k_{2} \neq 0$.

Portanto $G\left(U^{a}\right)$ não é uma $k$-álgebra de Lie sobre $k$.

b) Seja a $k$-álgebra

$$
G\left(U^{b}\right)=\mathfrak{s l}_{3}(k) \oplus k t_{3} \oplus U^{b} .
$$

Vejamos se ela é uma $k$-álgebra de Lie verificando a identidade de Jacobi, para isso, definamos explicitamente $\mathfrak{s l}_{3}(k)$-morfismo $\varphi: \Lambda^{2}\left(U^{b}\right) \rightarrow \mathfrak{s l}_{3}(k) \oplus k t_{3}$. De fato, como $U^{b}:=\left(V_{0} \oplus V_{3}^{*}\right) \oplus\left(V_{3} \wedge V_{0}\right)$ (ver tab. 3.16), pelo exemplo 1.21.5:

$$
\begin{aligned}
\Lambda^{2}\left(U^{b}\right) \simeq & \Lambda^{2}\left(V_{0} \oplus V_{3}^{*}\right) \oplus\left(\left(V_{0} \oplus V_{3}^{*}\right) \otimes_{k}\left(V_{3} \wedge V_{0}\right)\right) \oplus \Lambda^{2}\left(V_{3} \wedge V_{0}\right) \\
\simeq & \left(V_{0} \wedge V_{0}\right) \oplus\left(V_{0} \otimes_{k} V_{3}^{*}\right) \oplus\left(V_{3}^{*} \wedge V_{3}^{*}\right) \oplus\left(V_{3} \wedge V_{0}\right) \otimes_{k} V_{0} \oplus \\
& \oplus\left(V_{3} \wedge V_{0}\right) \otimes_{k} V_{3}^{*} \oplus\left(V_{3} \wedge V_{0}\right) \wedge\left(V_{3} \wedge V_{0}\right) .
\end{aligned}
$$

Agora, consideremos os seguintes $\mathfrak{s l}_{3}(k)$-submódulos de $U^{b} \wedge U^{b}$ :

- $U_{1}^{b}=V_{0} \wedge V_{0}=0$

- $U_{2}^{b}:=\left(V_{0} \otimes_{k} V_{3}^{*}\right) \simeq\left(V_{0} \wedge V_{3}^{*}\right) \simeq V_{3}^{*} \wedge V_{0} \simeq V_{3}^{*}$.

- $U_{3}^{b}:=V_{3}^{*} \wedge V_{3}^{*} \simeq V_{3}^{*}$.

- $U_{4}^{b}:=\left(V_{3} \wedge V_{0}\right) \otimes_{k} V_{0} \simeq\left(V_{3} \wedge V_{0}\right) \wedge V_{0} \simeq V_{0} \wedge\left(V_{3} \wedge V_{0}\right)$.

- $U_{5}^{b}:=V_{3}^{*} \otimes_{k}\left(V_{3} \wedge V_{0}\right) \simeq V_{3}^{*} \wedge\left(V_{3} \wedge V_{0}\right) \simeq\left(V_{3} \wedge V_{0}\right) \wedge V_{3}^{*}$.

- $U_{6}^{b}:=\left(V_{3} \wedge V_{0}\right) \wedge\left(V_{3} \wedge V_{0}\right)$.

Pelo mesmos argumentos como no caso anterior, temos que $\varphi\left(U_{i}^{b}\right)$ é um $\mathfrak{s l}_{3}(k)$-submódulos de $\mathfrak{s l}_{3}(k) \oplus k t_{3}$ para $i=1,2,3,4,6$, assim: $U_{i}^{b} \rightarrow k t_{3}$, ou $U_{i}^{b} \rightarrow 0$, ou $U_{i}^{b} \rightarrow \mathfrak{s l}_{3}(k)$. Suponhamos que $U_{i}^{b} \rightarrow k t_{3}\left(U_{i}^{b} \rightarrow \mathfrak{s l}_{3}(k)\right)$ para $i=2,3,4,6$. Então, para $i=2,3$ temos que $U_{2}^{b}, U_{3}^{b}$ são $\mathfrak{s l}_{3}(k)$-módulos irredutíveis, então pelo lema de Schur eles são isomorfos a $k\left(\mathfrak{s l}_{3}(k)\right)$, o qual 
é absurdo. Portanto $\left.\varphi\right|_{U_{i}^{b}}=0$ para $i=2,3$.

Para $i=4, U_{4}^{b}=\left\langle a \wedge z, a_{1} \wedge z, a_{2} \wedge z, a_{3} \wedge z\right\rangle$, onde a ação de $\mathfrak{s l}_{3}(k)$ sobre $U_{4}^{b}$ é dada na tabela 4.2 :

\begin{tabular}{|c||c|c|c|c|c|c|c|c|}
\hline & $h_{1}$ & $h_{2}$ & $e_{12}$ & $e_{13}$ & $e_{23}$ & $e_{21}$ & $e_{31}$ & $e_{32}$ \\
\hline \hline$a \wedge z$ & 0 & 0 & 0 & 0 & 0 & 0 & 0 & 0 \\
\hline$a_{1} \wedge z$ & $a_{1} \wedge z$ & 0 & $a_{2} \wedge z$ & $a_{3} \wedge z$ & $\beta(a \wedge z)$ & 0 & 0 & $\kappa(a \wedge z)$ \\
\hline$a_{2} \wedge z$ & $a_{2} \wedge z$ & $a_{2} \wedge z$ & 0 & $\gamma(a \wedge z)$ & $a_{3} \wedge z$ & $a_{1} \wedge z$ & $\kappa(a \wedge z)$ & 0 \\
\hline$a_{3} \wedge z$ & 0 & $a_{3} \wedge z$ & $\gamma(a \wedge z)$ & 0 & 0 & $\beta(a \wedge z)$ & $a_{1} \wedge z$ & $a_{2} \wedge z$ \\
\hline
\end{tabular}

Tabela 4.2: $O\left(\mathfrak{s l}_{3}(k), 2\right)$-módulo $\left(V_{3} \wedge V_{0}\right) \wedge V_{0}$

Da tabela 4.2 , note que, $W:=\langle a \wedge z\rangle$ é um $\left(\mathfrak{s l}_{3}(k), 2\right)$-submódulo de $U_{4}^{b}$, e $U_{4}^{b} / W \simeq V_{3}$. Além do mais, $0=\varphi\left(a_{2} \wedge z\right) . e_{13}=\varphi\left(\left(a_{2} \wedge z\right) e_{13}\right)=$ $\varphi(\gamma(a \wedge z))=\gamma \varphi(a \wedge z)$, como $\gamma \neq 0$, então $\varphi(a \wedge z)=0$, portanto $W \subseteq \operatorname{Ker}\left(\left.\varphi\right|_{U_{4}^{b}}\right)$. Logo, $\operatorname{Ker}\left(\left.\varphi\right|_{U_{4}^{b}}\right) / W$ é submódulo de $U_{4}^{b} / W \simeq V_{3}$, como $V_{3}$ é irredutível, temos que $\operatorname{Ker}\left(\left.\varphi\right|_{U_{4}^{b}}\right)=W$ ou $\operatorname{Ker}\left(\left.\varphi\right|_{U_{4}^{b}}\right)=U_{4}^{b}$. Por outro lado, como $\left.\varphi\right|_{U_{4}^{b}}: U_{4}^{b} \rightarrow k t_{3}$ é um $\mathfrak{s l}_{3}(k)$-morfismo sobrejetor, temos que $U_{4}^{b} / \operatorname{Ker}\left(\left.\varphi\right|_{U_{4}^{b}}\right) \simeq k$. Assim, se $\operatorname{ker}\left(\left.\varphi\right|_{U_{4}^{b}}\right)=W$, então $V_{3} \simeq k$ ou, se $\operatorname{Ker}\left(\left.\varphi\right|_{U_{4}^{b}}\right)=U_{4}^{b}$, então $0 \simeq k$. Uma contradição. Agora, se $U_{4}^{b} \rightarrow \mathfrak{s l}_{3}(k)$, então do $\mathfrak{s l}_{3}(k)$-morfismo sobrejetor $U_{4}^{b} \rightarrow \mathfrak{s l}_{3}(k)$ obtemos $\operatorname{dim}_{k}\left(U_{4}^{b}\right) \geq 8$, o qual é absurdo. Assim, $\left.\varphi\right|_{U_{4}^{b}}=0$.

Para $i=6$ temos $U_{6}^{b}=\left\langle p_{1}, p_{2}, p_{3}, p_{12}, p_{13}, p_{23}\right\rangle$, onde $p_{i}:=a \wedge a_{i}, p_{i j}:=a_{i} \wedge a_{j}$ com $1 \leq i<j \leq 3$. (ver tab. 4.3).

\begin{tabular}{|c||c|c|c|c|c|c|c|c|}
\hline & $h_{1}$ & $h_{2}$ & $e_{12}$ & $e_{13}$ & $e_{23}$ & $e_{21}$ & $e_{31}$ & $e_{32}$ \\
\hline \hline$p_{1}$ & $p_{1}$ & 0 & $p_{2}$ & $p_{3}$ & 0 & 0 & 0 & 0 \\
\hline$p_{2}$ & $p_{2}$ & $p_{2}$ & 0 & 0 & $p_{3}$ & $p_{1}$ & 0 & 0 \\
\hline$p_{3}$ & 0 & $p_{3}$ & 0 & 0 & 0 & 0 & $p_{1}$ & $p_{2}$ \\
\hline$p_{12}$ & 0 & $p_{12}$ & 0 & $p_{32}+\gamma p_{1}$ & $p_{13}+\beta p_{2}$ & 0 & $\kappa p_{1}$ & $\kappa p_{2}$ \\
\hline$p_{13}$ & $p_{13}$ & $p_{13}$ & $p_{23}+\gamma p_{1}$ & 0 & $\beta p_{3}$ & $\beta p_{1}$ & 0 & $p_{12}+\kappa p_{3}$ \\
\hline$p_{23}$ & $p_{23}$ & 0 & $\gamma p_{2}$ & $\gamma p_{3}$ & 0 & $p_{13}+\beta p_{2}$ & $p_{21}+\gamma p_{3}$ & 0 \\
\hline
\end{tabular}

Tabela 4.3: $O\left(\mathfrak{s l}_{3}(k), 2\right)$-módulo $\Lambda^{2}\left(V_{3} \wedge V_{0}\right)$

Note que, $S:=\left\langle p_{1}, p_{2}, p_{3}\right\rangle \simeq V_{3}$ é um $\left(s l_{3}(k), 2\right)$-submódulo de $U_{6}^{b}$ e, $U_{6}^{b} / S \simeq$ $V_{3}^{*}$. Também, se $\left.\varphi\right|_{U_{6}^{b}}: U_{6}^{b} \rightarrow k t_{3}$, temos que $S \subset \operatorname{Ker}\left(\left.\varphi\right|_{U_{6}^{b}}\right)$. De fato, $0=\varphi\left(p_{i}\right) h_{i}=\varphi\left(p_{i} \cdot h_{i}\right)=\varphi\left(p_{i}\right)$. Então $\operatorname{Ker}(\varphi) / S \leq U_{6}^{b} / S \simeq V_{3}^{*}$, e assim $\operatorname{Ker}\left(\left.\varphi\right|_{U_{6}^{b}}\right)=U_{6}^{b}$, ou $\operatorname{Ker}\left(\left.\varphi\right|_{U_{6}^{b}}\right)=S$, pelo mesmo argumento do caso anterior chegamos a um absurdo. Agora, se temos $\left.\varphi\right|_{U_{6}^{b}}: U_{6}^{b} \rightarrow \mathfrak{s l}_{3}(k)$, obtemos que $\operatorname{dim}_{k} U_{6}^{b} \geq 8$, o qual é falso. Portanto $\left.\varphi\right|_{U_{6}^{b}}=0$. Para $i=5$, temos $U_{5}^{b}=\left\langle z_{i} \wedge a, z_{i} \wedge a_{j}: 1 \leq i<j \leq 3\right\rangle$, onde a $\mathfrak{s l}_{3}(k)$-ação sobre $U_{5}^{b}$ é dada na tabela 4.4. 


\begin{tabular}{|c||c|c|c|c|c|c|c|c|}
\hline & $h_{1}$ & $h_{2}$ & $e_{12}$ & $e_{13}$ & $e_{23}$ & $e_{21}$ & $e_{31}$ & $e_{32}$ \\
\hline \hline$q_{1}$ & $q_{1}$ & 0 & 0 & 0 & 0 & $q_{2}$ & $q_{3}$ & 0 \\
\hline$q_{2}$ & $q_{2}$ & $q_{2}$ & $q_{1}$ & 0 & 0 & 0 & 0 & $q_{3}$ \\
\hline$q_{3}$ & 0 & $q_{3}$ & 0 & $q_{1}$ & $q_{2}$ & 0 & 0 & 0 \\
\hline$q_{11}$ & 0 & 0 & $q_{12}$ & $q_{13}$ & $\beta q_{1}$ & $q_{21}$ & $q_{31}$ & $\kappa q_{1}$ \\
\hline$q_{22}$ & 0 & 0 & $q_{12}$ & $\gamma q_{2}$ & $q_{23}$ & $q_{21}$ & 0 & $q_{32}$ \\
\hline$q_{12}$ & 0 & $q_{12}$ & 0 & $\gamma q_{1}$ & $q_{13}$ & $q_{22}+q_{11}$ & $q_{32}+\kappa q_{1}$ & 0 \\
\hline$q_{13}$ & $q_{13}$ & $q_{13}$ & $\gamma q_{1}$ & 0 & 0 & $q_{23}+\beta q_{1}$ & $q_{33}+q_{11}$ & $q_{12}$ \\
\hline$q_{23}$ & $q_{23}$ & 0 & $q_{13}+\gamma q_{2}$ & 0 & $\beta q_{2}$ & $\beta q_{2}$ & $q_{21}$ & $q_{33}+q_{22}$ \\
\hline$q_{21}$ & 0 & $q_{21}$ & $q_{11}+q_{22}$ & $q_{23}$ & $\beta q_{2}$ & 0 & 0 & $q_{31}+\kappa q_{2}$ \\
\hline$q_{31}$ & $q_{31}$ & $q_{31}$ & $q_{32}$ & $q_{11}+q_{33}$ & $q_{21}+\beta q_{3}$ & 0 & 0 & 0 \\
\hline$q_{32}$ & $q_{32}$ & 0 & 0 & $q_{12}+\gamma q_{3}$ & $q_{22}+q_{33}$ & $q_{31}$ & $\kappa q_{3}$ & 0 \\
\hline$q_{33}$ & 0 & 0 & $\gamma q_{3}$ & $q_{13}$ & $q_{23}$ & $\beta q_{3}$ & $q_{31}$ & $q_{32}$ \\
\hline
\end{tabular}

Tabela 4.4: $O\left(\mathfrak{s l}_{3}(k), 2\right)$-módulo $V_{3}^{*} \wedge\left(V_{3} \wedge V_{0}\right)$

onde: $q_{i}:=z_{i} \wedge a, q_{i i}:=z_{i} \wedge a_{i}$, para $i=1,2,3 ; q_{12}:=z_{1} \wedge a_{2}, q_{13}:=z_{1} \wedge a_{3}$, $q_{23}:=z_{2} \wedge a_{3}, q_{21}:=z_{2} \wedge a_{1}, q_{31}:=z_{3} \wedge a_{1}, q_{32}:=z_{3} \wedge a_{2}$.

Seja $W:=\left\langle q_{1}, q_{2}, q_{3}\right\rangle$, da tabela 4.4 vemos que

$$
U_{5}^{b} / W \simeq V_{3}^{*} \otimes_{k} V_{3} \simeq V_{3}^{*} \wedge V_{3} \underset{\simeq}{\stackrel{f}{\longrightarrow}} \mathfrak{s l}_{3}(k) \oplus k t_{3}
$$

onde $f$ é dada no exemplo 3.1. Portanto, podemos definir $\left.\varphi\right|_{U_{5}^{b}}:=f \circ p$, onde $p: U_{5}^{b} \longrightarrow U_{5}^{b} / W$ é o morfismo quociente. Agora, pela tabela 3.16 encontramos que:

$J\left(a_{1}, z_{1}, z_{3}\right)=\left[a_{1},\left[z_{1}, z_{3}\right]\right]+\left[z_{1},\left[a_{1}, z_{3}\right]\right]+\left[z_{3},\left[a_{1}, z_{1}\right]\right]=z_{1} e_{31}+z_{3}\left(h_{2}+t_{3}\right)=$ $=z_{3}+z_{3}+z_{3}=z_{3} \neq 0$.

Portanto, $G\left(U^{b}\right)$ não é uma álgebra de Lie sobre $k$.

c) Pelo exemplo 1.21.5 achamos que:

$$
\begin{aligned}
\Lambda^{2}\left(U^{c}\right) \simeq & \left(V_{0} \wedge V_{0}\right) \oplus\left(V_{0} \otimes_{k} V_{3}\right) \oplus\left(V_{3} \wedge V_{3}\right) \oplus\left(\left(V_{3}^{*} \wedge V_{0}\right) \otimes V_{0}\right) \oplus \\
& \oplus\left(\left(V_{3}^{*} \wedge V_{0}\right) \otimes_{k} V_{3}\right) \oplus\left(\left(V_{3}^{*} \wedge V_{0}\right) \wedge\left(V_{3}^{*} \wedge V_{0}\right)\right) .
\end{aligned}
$$

Assim, temos os seguintes $\mathfrak{s l}_{3}(k)$-submódulos de $\Lambda^{2}\left(U^{c}\right)$ :

- $U_{1}^{c}=V_{0} \wedge V_{0}=0$

- $U_{2}^{c}:=V_{0} \otimes_{k} V_{3} \simeq V_{0} \wedge V_{3} \simeq V_{3} \wedge V_{0} \simeq V_{3}$.

- $U_{3}^{c}:=V_{3} \wedge V_{3} \simeq V_{3}$.

- $U_{4}^{c}:=\left(V_{3}^{*} \wedge V_{0}\right) \otimes V_{0} \simeq\left(V_{3}^{*} \wedge V_{0}\right) \wedge V_{0} \simeq V_{0} \wedge\left(V_{3}^{*} \wedge V_{0}\right)$.

- $U_{5}^{c}:=V_{3} \otimes_{k}\left(V_{3}^{*} \wedge V_{0}\right) \simeq V_{3} \wedge\left(V_{3}^{*} \wedge V_{0}\right) \simeq\left(V_{3}^{*} \wedge V_{0}\right) \wedge V_{3}$.

- $U_{6}^{c}:=\left(V_{3}^{*} \wedge V_{0}\right) \wedge\left(V_{3}^{*} \wedge V_{0}\right)$.

Vejamos como é $\left.\varphi\right|_{U_{i}^{c}}$ para todo $i=2,3,4,5,6$. Se $\left.\varphi\right|_{U_{i}^{c}}: U_{i}^{c} \longrightarrow k t_{3}$ ou $\left.\varphi\right|_{U_{i}^{c}}: U_{i}^{c} \longrightarrow \mathfrak{s l}_{3}(k)$ para $i=2,3$ se segue, pelo lema de Schur, que $U_{i}^{c} \simeq k t_{3}$ ou $U_{i}^{c} \simeq \mathfrak{s l}_{3}(k)$, já que $U_{i}^{c}$ é irredutível. Uma contradição. Portanto 
$\left.\varphi\right|_{U_{i}^{c}}=0$ para $i=2,3$. Para $i=4$ suponhamos que $\left.\varphi\right|_{U_{4}^{c}}: U_{4}^{c} \longrightarrow k t_{3}$, então $U_{4}^{c} / \operatorname{Ker}\left(\left.\varphi\right|_{U_{4}^{c}}\right) \simeq k t_{3}$. Por outro lado, se $: s_{i}:=b_{i} \wedge v$ e, $s:=b \wedge v$, então $U_{4}^{c}=\left\langle s, s_{i}: i=1,2,3\right\rangle \subseteq \operatorname{Ker}\left(\left.\varphi\right|_{U_{4}^{c}}\right)$. De fato, $0=\varphi\left(s_{1}\right) \cdot e_{23}=\varphi\left(s_{1} e_{23}\right)=$ $\pi \varphi(s)$ então, $\pi=0$ ou $\varphi(s)=0$, como $\pi \neq 0$, então $\varphi(s)=0$. Também: $0=\varphi\left(s_{i}\right) \cdot h_{i}=\varphi\left(s_{i} \cdot h_{i}\right)=\varphi\left(s_{i}\right), i=1,2 ; 0=h_{2} \varphi\left(s_{3}\right) \cdot h_{2}=\varphi\left(s_{3} h_{2}\right)=\varphi\left(s_{3}\right)$. Assim, temos que $0=\operatorname{Im}\left(\left.\varphi\right|_{U_{4}^{c}}\right)=k t_{3}$, uma contradição.

Agora, $\left.\varphi\right|_{U_{4}^{c}}: U_{4}^{c} \longrightarrow \mathfrak{s l}_{3}(k)$ é um $\mathfrak{s l}_{3}(k)$-morfismo sobrejetor, $\operatorname{assim}_{\operatorname{dim}}\left(U_{4}^{c}\right) \geq$ 8 , o qual é falso. Portanto $\left.\varphi\right|_{U_{4}^{c}}=0$.

Para $i=6$, temos $U_{c}^{6}=\left\langle f_{1}, f_{2}, f_{3}, f_{12}, f_{13}, f_{23}\right\rangle$ (ver tab. 4.5 abaixo), onde $f_{i}:=b \wedge b_{i}$ para $1,2,3$ e, $f_{i j}:=b_{i} \wedge b_{j}$ com $1 \leq i<j \leq 3$.

\begin{tabular}{|c||c|c|c|c|c|c|c|c|}
\hline & $h_{1}$ & $h_{2}$ & $e_{12}$ & $e_{13}$ & $e_{23}$ & $e_{21}$ & $e_{31}$ & $e_{32}$ \\
\hline \hline$f_{1}$ & $f_{1}$ & 0 & 0 & 0 & 0 & $f_{2}$ & $f_{3}$ & 0 \\
\hline$f_{2}$ & $f_{2}$ & $f_{1}$ & 0 & 0 & 0 & 0 & 0 & $f_{3}$ \\
\hline$f_{3}$ & 0 & $f_{3}$ & 0 & $f_{1}$ & $f_{2}$ & 0 & 0 & 0 \\
\hline$f_{12}$ & 0 & $f_{12}$ & 0 & $\pi f_{1}$ & $\pi f_{2}$ & 0 & $\xi f_{2}+f_{32}$ & $\lambda f_{2}+f_{13}$ \\
\hline$f_{13}$ & $f_{13}$ & $f_{13}$ & $\lambda f_{1}$ & 0 & $\pi f_{3}+f_{12}$ & $\xi f_{1}+f_{23}$ & 0 & $\lambda f_{3}$ \\
\hline$f_{23}$ & $f_{23}$ & 0 & $\lambda f_{2}+f_{13}$ & $\pi f_{12}$ & 0 & $\xi f_{2}$ & $\xi f_{3}$ & 0 \\
\hline
\end{tabular}

Tabela 4.5: $O\left(\mathfrak{s l}_{3}(k), 2\right)$-módulo $\Lambda^{2}\left(V_{3}^{*} \curlywedge V_{0}\right)$

Suponhamos que $\left.\varphi\right|_{U_{6}^{c}}: U_{6}^{c} \rightarrow k t_{3}\left(\left.\varphi\right|_{U_{6}^{c}}: U_{6}^{c} \rightarrow \mathfrak{s l}_{3}(k)\right)$.

Seja $W:=\left\langle f_{1}, f_{2}, f_{3}\right\rangle$, da tabela 4.5 vemos que $W$ é submódulo de $U_{6}^{c}$, que é isomorfo a $V_{3}^{*}$. Logo, o $\mathfrak{s l}_{3}(k)$-morfismo $\left.\varphi\right|_{U_{6}^{c}} \circ \iota: W \rightarrow k t_{3}$

$\left(\left.\varphi\right|_{U_{6}^{c} \circ} \circ: W \rightarrow \mathfrak{s l}_{3}(k)\right)$ é um isomorfismo, pelo lema de Shur. Portanto, $V_{3}^{*} \simeq k t_{3}\left(V_{3}^{*} \simeq \mathfrak{s l}_{3}(k)\right)$. Uma contradição. Assim, $\left.\varphi\right|_{U_{6}^{c}} \circ \iota=0$, portanto $W \subseteq \operatorname{Ker}\left(\left.\varphi\right|_{U_{6}^{c}}\right)$, logo $\operatorname{Ker}\left(\left.\varphi\right|_{U_{6}^{c}}\right) / W \leqslant U_{6}^{c} / W \simeq V_{3}$, segue-se que $\operatorname{Ker}\left(\left.\varphi\right|_{U_{6}^{c}}\right)=W$ ou $\operatorname{Ker}\left(\left.\varphi\right|_{U_{6}^{c}}\right)=U_{6}^{c}$. Portanto, $0 \simeq k t_{3}\left(\mathfrak{s l}_{3}(k)\right)$ ou $V_{3} \simeq k t_{3}\left(\mathfrak{s l}_{3}(k)\right)$, o qual é absurdo. Assim, $\left.\varphi\right|_{U_{6}^{c}}=0$.

Para $i=5$, temos que $W:=\left\langle v_{1} \wedge b, v_{2} \wedge b, v_{3} \wedge b\right\rangle$ é submódulo de $U_{5}^{c} \mathrm{e}$

$$
U_{5}^{c} / W \simeq V_{3} \wedge V_{3}^{*} \stackrel{g}{\simeq} \mathfrak{s l}_{3}(k) \oplus k t_{3} .
$$

Podemos definir, $\left.\varphi\right|_{U_{5}^{c}}=g \circ p$, onde $p: U_{5}^{b} \longrightarrow U_{5}^{b} / W$ é o morfismo quociente e $g$ como no exemplo 3.1. Assim, pela tabela 3.20 obtemos:

$J\left(b_{1}, v_{1}, v_{2}\right)=\left[b_{1},\left[v_{1}, v_{2}\right]\right]+\left[v_{1},\left[b_{1}, v_{2}\right]\right]+\left[v_{2},\left[b_{1}, v_{1}\right]\right]=v_{1} e_{12}+v_{2}\left(h_{2}+t_{3}\right)=$ $=v_{2}+v_{2}+v_{2}=v_{2} \neq 0$.

Portanto, $G\left(U^{c}\right)$ não é uma álgebra de Lie sobre $k$.

d) $G\left(U^{d}\right):=\mathfrak{s l}_{3}(k) \oplus k t_{3} \oplus U^{d}$ é uma $k$-álgebra. Vejamos se ela é uma $k$-álgebra de Lie. Para isso, precisamos definir explicitamente o $\mathfrak{s l}_{3}(k)$-morfismo

$$
\varphi: \Lambda^{2}\left(U^{d}\right) \rightarrow \mathfrak{s l}_{3}(k) \oplus k t_{3} .
$$

De fato, como $U^{d}:=\left(V_{3}^{*} \curlywedge V_{0}\right) \oplus\left(V_{3} \wedge V_{0}\right)$ (Ver tab. 3.14) então: 


$$
\Lambda^{2}\left(U^{d}\right) \simeq \Lambda^{2}\left(V_{3}^{*} \curlywedge V_{0}\right) \oplus\left(V_{3}^{*} \curlywedge V_{0}\right) \otimes_{k}\left(V_{3} \curlywedge V_{0}\right) \oplus \Lambda^{2}\left(V_{3} \curlywedge V_{0}\right)
$$

Agora, consideremos os seguintes $\mathfrak{s l}_{3}(k)$-submódulos de $U^{d} \wedge U^{d}$ :

- $U_{1}^{d}:=\left(V_{3} \wedge V_{0}\right) \wedge\left(V_{3} \wedge V_{0}\right)$

- $U_{2}^{d}:=\left(V_{3}^{*} \wedge V_{0}\right) \wedge\left(V_{3}^{*} \wedge V_{0}\right)$

- $U_{3}^{d}:=\left(V_{3}^{*} \wedge V_{0}\right) \otimes_{k}\left(V_{3} \wedge V_{0}\right) \simeq\left(V_{3} \wedge V_{0}\right) \wedge\left(V_{3}^{*} \wedge V_{0}\right) \simeq\left(V_{3}^{*} \wedge V_{0}\right) \wedge\left(V_{3} \wedge V_{0}\right)$

Então $\varphi\left(U_{i}^{d}\right)$ é um $\mathfrak{s l}_{3}(k)$-submódulos de $\mathfrak{s l}_{3}(k) \oplus k t_{3}$ para $i=1,2,3$. Assim: $U_{i}^{d} \rightarrow k t_{3}$, ou $U_{i}^{d} \rightarrow 0$, ou $U_{i}^{d} \rightarrow \mathfrak{s l}_{3}(k)$. Suponhamos que $U_{i}^{d} \stackrel{\varphi}{\longrightarrow} k t_{3}$ $\left(U_{i}^{d} \stackrel{\varphi}{\longrightarrow} \mathfrak{s l}_{3}(k)\right)$ para $i=1,2$.

Para $i=1$, com o mesmo argumento aplicado a $U_{6}^{b}$ do caso $b$. (ver tab. 4.3) temos que: $\left.\varphi\right|_{U_{1}^{d}}=0$.

Para $i=2$, aplicando o mesmo argumento usado em $U_{6}^{c}$ de $c$. (ver tab. 4.5) encontramos que $U_{4}^{d} \stackrel{\varphi}{\longrightarrow} 0$ Para $i=3$.

Tem-se $U_{3}^{d}=\left\langle l, l_{i}, l_{i i}, l_{i j}, s_{i}: 1 \leq i<j \leq 3\right\rangle$ onde $l:=u \wedge x, l_{i}:=u_{i} \wedge x$, $l_{i i}:=u_{i} \wedge x_{i}, l_{i j}:=u_{i} \wedge x_{j}$ e, $s_{i}:=x_{i} \wedge u$, com $1 \leq i<j \leq 3$, (ver tab. 4.6).

\begin{tabular}{|c||c|c|c|c|c|c|c|c|}
\hline & $h_{1}$ & $h_{2}$ & $e_{12}$ & $e_{13}$ & $e_{23}$ & $e_{21}$ & $e_{31}$ & $e_{32}$ \\
\hline \hline$l_{1}$ & $l_{1}$ & 0 & 0 & 0 & $\pi l$ & $l_{2}$ & $l_{3}$ & $\lambda l$ \\
\hline$l_{2}$ & $l_{2}$ & $l_{2}$ & $l_{1}$ & $\pi l$ & 0 & 0 & $\xi l$ & $l_{3}$ \\
\hline$l_{3}$ & 0 & $l_{3}$ & $\lambda l$ & $l_{1}$ & $l_{2}$ & $\xi l$ & 0 & 0 \\
\hline$l_{11}$ & 0 & 0 & $l_{12}$ & $l_{13}$ & $\beta l_{1}+\pi s_{1}$ & $l_{21}$ & $l_{31}$ & $\alpha l_{1}+\lambda s_{1}$ \\
\hline$l_{22}$ & 0 & 0 & $l_{12}$ & $\gamma l_{2}+\pi s_{2}$ & $l_{23}$ & $l_{21}$ & $\xi s_{2}+\alpha l_{2}$ & $l_{32}$ \\
\hline$l_{12}$ & 0 & $l_{12}$ & 0 & $\gamma l_{1}$ & $l_{13}+\pi s_{2}$ & $l_{22}+l_{11}$ & $l_{32}+\alpha l_{1}$ & $\lambda s_{2}$ \\
\hline$l_{13}$ & $l_{13}$ & $l_{13}$ & $\gamma l_{1}$ & 0 & $\pi s_{3}$ & $l_{23}+\beta l_{2}$ & $l_{33}+l_{11}$ & $l_{12}+\lambda s_{3}$ \\
\hline$l_{23}$ & $l_{23}$ & 0 & $l_{13}+\gamma l_{2}$ & $\pi s_{3}$ & 0 & $\beta l_{2}$ & $l_{21}+\xi s_{3}$ & $l_{33}+l_{22}$ \\
\hline$l_{21}$ & 0 & $l_{21}$ & $l_{11}+l_{22}$ & $l_{23}+\pi s_{1}$ & $\beta l_{2}$ & 0 & $\xi s_{1}$ & $l_{31}+\alpha l_{2}$ \\
\hline$l_{31}$ & $l_{31}$ & $l_{31}$ & $l_{32}+\lambda s_{1}$ & $l_{11}+l_{33}$ & $l_{21}+\beta l_{3}$ & $\xi s_{1}$ & 0 & $\alpha l_{3}$ \\
\hline$l_{32}$ & $l_{32}$ & 0 & $\lambda s_{2}$ & $l_{12}+\gamma l_{3}$ & $l_{22}+l_{33}$ & $l_{31}+\xi s_{2}$ & $\alpha l_{3}$ & 0 \\
\hline$l_{33}$ & 0 & 0 & $\gamma l_{3}+\lambda s_{3}$ & $l_{13}$ & $l_{23}$ & $\xi s_{3}+\beta l_{3}$ & $l_{31}$ & $l_{32}$ \\
\hline$l$ & 0 & 0 & 0 & 0 & 0 & 0 & 0 & 0 \\
\hline$s_{1}$ & $s_{1}$ & 0 & $s_{2}$ & $s_{3}$ & $\beta l$ & 0 & 0 & $\alpha l$ \\
\hline$s_{2}$ & $s_{2}$ & $s_{2}$ & 0 & $\gamma l$ & $s_{3}$ & $s_{1}$ & $\alpha l$ & 0 \\
\hline$s_{3}$ & 0 & $s_{3}$ & $\gamma l$ & 0 & 0 & $\beta l$ & $s_{1}$ & $s_{2}$ \\
\hline
\end{tabular}

Tabela 4.6: $O\left(\mathfrak{s l}_{3}(k), 2\right)$-módulo $\left(V_{3}^{*} \curlywedge V_{0}\right) \wedge\left(V_{3} \wedge V_{0}\right)$

Da tabela 4.6 , observamos que $W:=\left\langle l, l_{1}, l_{2}, l_{3}, s_{1}, s_{2}, s_{3}\right\rangle$ é um submódulo de $U_{3}^{d}$, e

$$
U_{3}^{d} / W \simeq V_{3} \wedge V_{3}^{*} \stackrel{f}{\simeq} \mathfrak{s l}_{3}(k) \oplus k t_{3} .
$$

$\left.\operatorname{logo} \varphi\right|_{U_{3}^{d}}:=f \circ p \operatorname{com} p: U_{3}^{d} \longrightarrow U_{3}^{d} / W$ o morfismo quociente e $f$ como no exemplo 3.1. Por outro lado, pela tabela 3.14 obtemos que

$J\left(u_{1}, x_{1}, x_{2}\right)=\left[x_{1},\left[u_{1}, x_{2}\right]\right]+\left[x_{2},\left[u_{1}, x_{1}\right]\right]=\left[x_{1}, e_{12}\right]+\left[x_{2}, h_{2}+t_{3}\right]=x_{2} \neq 0$.

Portanto, temos que $G\left(U^{d}\right)$ não é uma álgebra de Lie sobre $k$. 
e) Como $U^{e}=V_{3}^{*} \oplus\left[V_{3} \curlywedge\left(V_{0} \curlywedge V_{0}\right)\right]$ (ver tab. 3.44). Achemos $\Lambda^{2}\left(U^{e}\right)$ :

$$
\Lambda^{2}\left(U^{e}\right) \simeq \Lambda^{2}\left(V_{3}^{*}\right) \oplus\left(V_{3}^{*} \otimes_{k}\left(V_{3} \curlywedge\left(V_{0} \curlywedge V_{0}\right)\right)\right) \oplus \Lambda^{2}\left(V_{3} \curlywedge\left(V_{0} \curlywedge V_{0}\right)\right)
$$

Agora, consideremos os seguintes $\mathfrak{s l}_{3}(k)$-submódulos de $U^{e} \wedge U^{e}$ :

- $U_{1}^{e}:=V_{3}^{*} \wedge V_{3}^{*} \simeq V_{3}^{*}$

- $U_{2}^{e}:=V_{3}^{*} \otimes_{k}\left(V_{3} \wedge\left(V_{0} \wedge V_{0}\right)\right) \simeq V_{3}^{*} \wedge\left(V_{3} \wedge\left(V_{0} \wedge V_{0}\right)\right)$.

- $U_{3}^{e}:=\left(V_{3} \wedge\left(V_{0} \wedge V_{0}\right)\right) \wedge\left(V_{3} \wedge\left(V_{0} \wedge V_{0}\right)\right)$

Vejamos como está definida $\left.\varphi\right|_{U_{i}^{e}}, i=1,2,3$. Se $\left.\varphi\right|_{U_{1}^{e}}: U_{1}^{e} \rightarrow k t_{3}$, então $V_{3}^{*} \simeq U_{1}^{e} \simeq k t_{3}\left(\mathfrak{s l}_{3}(k)\right)$. Uma contradição. Portanto $\left.\varphi\right|_{U_{1}^{e}}=0$. Seja,

$U_{3}^{e}=\left\langle n, n_{i}, m_{i}, m_{i j}: 1 \leq i<j \leq 3\right\rangle$, onde $n:=x \wedge y, n_{i}:=x \wedge x_{i}, m_{i}:=$ $y \wedge x_{i}, m_{i j}:=x_{i} \wedge x_{j}$, para $1 \leq i<j \leq 3$. Usando a tabela 3.8 se pode construir a tabela para $U_{3}^{e}$ (ver página seguinte tabela 4.8). Da tabela 4.8, vemos que $W:=\left\langle n, m_{i}, n_{i}: i=1,2,3\right\rangle$ é um $\mathfrak{s l}_{3}(k)$-submódulo de $U_{3}^{e}$ e $U_{3}^{e} / W=\left\langle m_{i j}+W: 1 \leq i<j \leq 3\right\rangle \simeq V_{3}$.

Se temos, $\left.\varphi\right|_{U_{3}^{e}}: U_{3}^{e} \rightarrow \mathfrak{s l}_{3}(k)$, então $U_{3}^{e} / W \rightarrow \mathfrak{s l}_{3}(k) /\left.\varphi\right|_{U_{3}^{e}}(W)$ é um $\mathfrak{s l}_{3}(k)$ morfismo sobrejetor, já que $\left.\varphi\right|_{U_{3}^{e}}$ é sobrejetor. Como $U_{3}^{e} / W$ é um $\mathfrak{s l}_{3}(k)$ módulos irredutivel, então $V_{3} \simeq \mathfrak{s l}_{3}(k) /\left.\varphi\right|_{U_{3}^{e}}(W)$, e pela irredutívilidade do $\mathfrak{s l}_{3}(k)$-módulo adjunto, tem-se $\left.\varphi\right|_{U_{3}^{e}}(W)=0$ ou $\left.\varphi\right|_{U_{3}^{e}}(W)=\mathfrak{s l}_{3}(k)$. Portanto, $V_{3} \simeq \mathfrak{s l}_{3}(k)$ ou $V_{3} \simeq 0$. Uma contradição.

Suponhamos que $\left.\varphi\right|_{U_{3}^{e}}: U_{3}^{e} \longrightarrow k t_{3}$, então pelos os mesmos argumentos do parágrafo anterior achamos que, $V_{3} \simeq k t_{3}$ ou $V_{3} \simeq 0$. Uma contradição. Portanto, $\left.\varphi\right|_{U_{3}^{e}}=0$. Por outro lado,

$$
U_{2}^{e}=\left\langle a_{i}, b_{i}, a_{i i}, a_{12}, a_{13}, a_{23}, a_{21}, a_{31}, a_{32}: i=1,2,3\right\rangle
$$

onde $a_{i}:=y_{i} \wedge x, b_{i}:=y_{i} \wedge y \mathrm{e}, a_{i i}:=y_{i} \wedge x_{i}$, para todo $i=1,2,3, a_{12}:=y_{1} \wedge x_{2}$, $a_{13}:=y_{1} \wedge x_{3}, a_{23}:=y_{2} \wedge x_{3}, a_{21}:=y_{2} \wedge x_{1}, a_{31}:=y_{3} \wedge x_{1}$, e $a_{32}:=y_{3} \wedge x_{2}$. Para achar $\left.\varphi\right|_{U_{2}^{e}}$, precisamos da ação de $\mathfrak{s l}_{3}(k)$ sobre $U_{2}^{e}$, veja a tabela 4.10 (ver páginas seguintes). Seja

$$
W:=\left\langle y_{i} \wedge x, y_{i} \wedge y: i=1,2,3\right\rangle \simeq\left(V_{3}^{*} \wedge V_{0}\right) \oplus\left(V_{3}^{*} \wedge V_{0}\right)
$$

é um submódulo de $U_{2}^{e}$ e da tabela 4.10. vemos que:

$$
U_{2}^{e} / W \simeq V_{3}^{*} \wedge V_{3} \stackrel{f}{\simeq} \mathfrak{s l}_{3}(k) \oplus k t_{3}
$$

$\left.\operatorname{logo} \varphi\right|_{U_{2}^{e}}:=f \circ p \operatorname{com} f$ definida como no exemplo 3.1 e, $p: U_{2}^{e} \longrightarrow U_{2}^{e} / W$. Além disso, da tabela 3.44:

$$
J\left(x_{1}, y_{1}, y_{2}\right)=\left[y_{1},\left[x_{1}, y_{2}\right]\right]+\left[y_{2},\left[x_{1}, y_{1}\right]\right]=\left[y_{1}, e_{21}\right]+\left[y_{2}, h_{2}+t_{3}\right]=y_{2} \neq 0 \text {. }
$$

Portanto, $G\left(U^{e}\right)$ não é uma álgebra de Lie sobre $k$. 


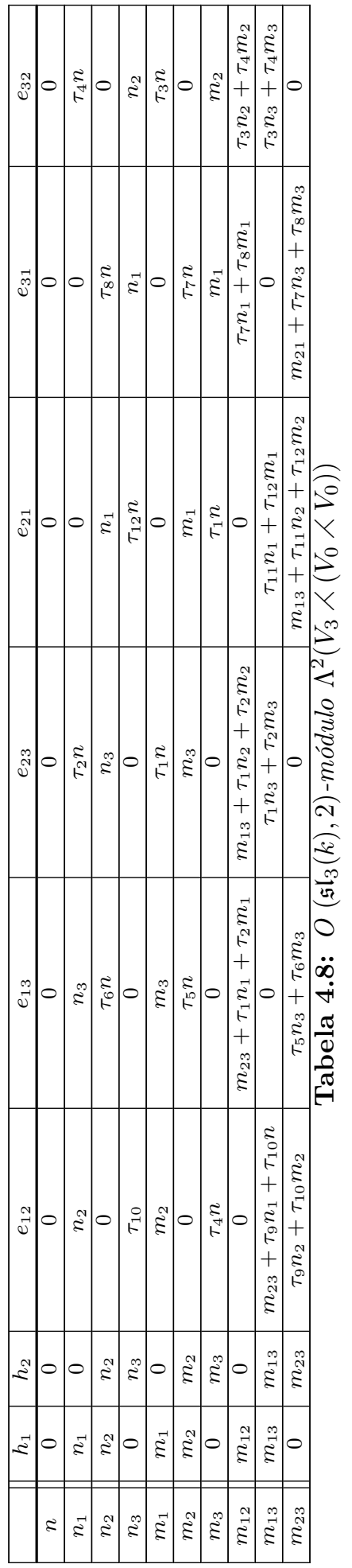




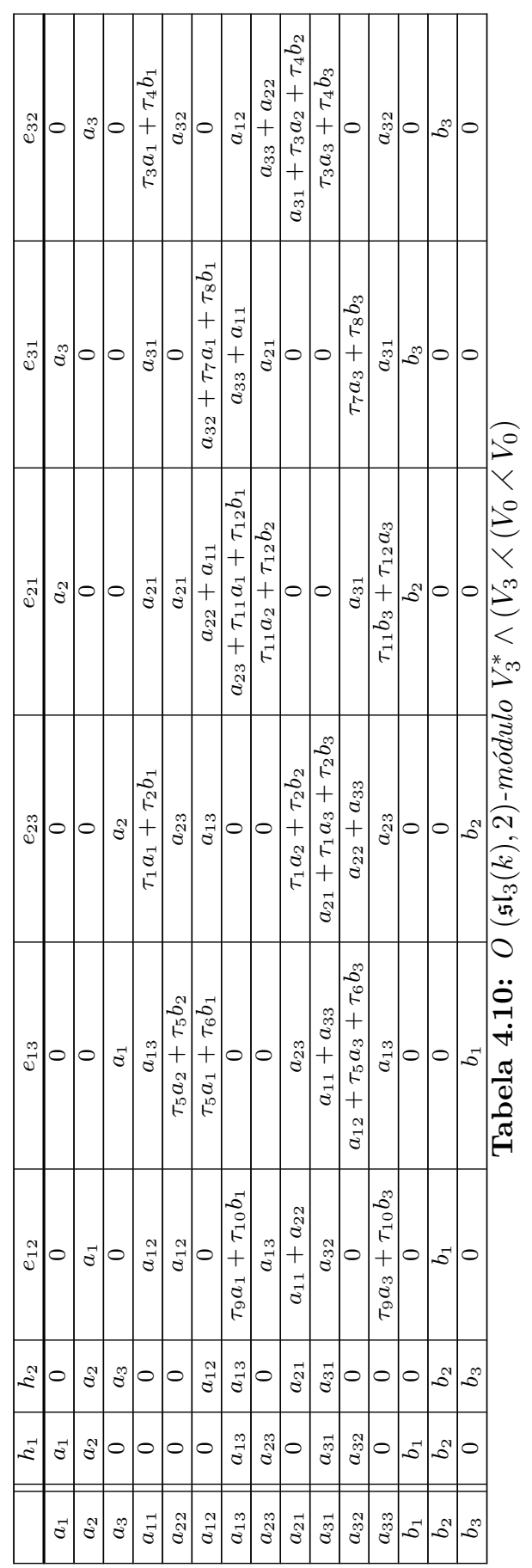


Finalmente,

f) Seja $U^{f}=V_{3} \oplus\left[V_{3}^{*} \curlywedge\left(V_{0} \curlywedge V_{0}\right)\right]$ (Ver tab. 26). Pelo exemplo 1.21.5 tem-se:

$$
\Lambda^{2}\left(U^{f}\right) \simeq \Lambda^{2}\left(V_{3}\right) \oplus\left(V_{3} \otimes_{k}\left[V_{3}^{*} \curlywedge\left(V_{0} \curlywedge V_{0}\right)\right]\right) \oplus \Lambda^{2}\left(V_{3}^{*} \curlywedge\left(V_{0} \curlywedge V_{0}\right)\right)
$$

e assim, temos os seguintes $\mathfrak{s l}_{3}(k)$-submódulos de $U^{f} \wedge U^{f}$ :

- $U_{1}^{f}:=V_{3} \wedge V_{3} \simeq V_{3}$

- $U_{2}^{f}:=V_{3} \wedge\left[V_{3}^{*} \wedge\left(V_{0} \wedge V_{0}\right)\right]$

- $U_{3}^{f}:=\left(V_{3}^{*} \wedge\left(V_{0} \wedge V_{0}\right)\right) \wedge\left(V_{3}^{*} \curlywedge\left(V_{0} \wedge V_{0}\right)\right)$

e novamente pelos mesmos argumentos usados em $U_{1}^{e}, U_{2}^{e}, U_{3}^{e}$ do caso $e$. anterior, tem-se:

$\left.\varphi\right|_{U_{1}^{f}}=0:$ já que, se $U_{1}^{f} \rightarrow k t_{3}$, ou $U_{1}^{f} \rightarrow \mathfrak{s l}_{3}(k)$, então pelo lema de Schur, $V_{3} \simeq k t_{3}$, ou $V_{3} \simeq \mathfrak{s l}_{3}(k)$, o qual é absurdo.

$\left.\varphi\right|_{U_{3}^{f}}=0:$ já que, se $U_{3}^{f} \rightarrow k t_{3}$, ou $U_{3}^{f} \rightarrow \mathfrak{s l}_{3}(k)$, encontramos que $U_{3}^{f}$ tem um $\mathfrak{s l}_{3}(k)$-submódulo $S:=\left\langle v \wedge g, v \wedge g_{1}, v \wedge g_{2}, v \wedge g_{3}, g \wedge g_{1}, g \wedge g_{2}, g \wedge g_{3}\right\rangle$ tal que: $U_{3}^{f} / W \simeq V_{3}^{*}$. Além disso, $U_{3}^{f} / S \rightarrow k t_{3} /\left.\varphi\right|_{U_{3}^{f}}(S)\left(U_{3}^{f} / S \rightarrow \mathfrak{s l}_{3}(k) /\left.\varphi\right|_{U_{3}^{f}}(S)\right)$ é um $\mathfrak{s l}_{3}(k)$-isomorfismo. Então $V_{3}^{*} \simeq k t_{3}\left(\mathfrak{s l}_{3}(k)\right)$ ou $V_{3}^{*} \simeq 0$ o qual é uma contradição. Agora, para $U_{2}^{f}$ temos que,

$$
W:=\left\langle v_{i} \wedge g, g_{i} \wedge v: i=1,2,3\right\rangle \simeq\left(V_{3} \wedge V_{0}\right) \oplus\left(V_{3} \wedge V_{0}\right)
$$

é um $\mathfrak{s l}_{3}(k)$-submódulo de $U_{2}^{f}$ e

$$
U_{2}^{f} / W \simeq V_{3} \wedge V_{3}^{*} \stackrel{g}{\simeq} \mathfrak{s l}_{3}(k) \oplus k t_{3}
$$

$\left.\log \varphi\right|_{U_{2}^{f}}:=g \circ p \operatorname{com} p: U_{2}^{f} \longrightarrow U_{2}^{f} / W$ e $g$ definida como no exemplo 3.1. $G\left(U^{f}\right)$ não é uma álgebra Lie sobre $k$, já que pela tabela 3.26 temos que :

$$
J\left(v_{1}, g_{1}, g_{2}\right)=g_{2} \neq 0 .
$$

Portanto, as $k$-álgebras $G\left(U^{j}\right)$ para $j=a, b, c, d, e, f$ não são $k$-álgebras de Lie.

Em conclusão, se $(L,[2])$ contém uma 2-subálgebra de Lie simples, então não existem 2 álgebras de Lie simple de dimensão 17 de posto toral 3, da forma $(17: 3,0,2,2,2,2,2,2,2)$, isto é:

Teorema 4.2. Não existem 2-álgebras de Lie $(L,[2])$, simples de dim $_{k}=17$ de posto toral 3, contendo a $\left(\mathfrak{s l}_{3}(k), 2\right)$ como 2-subálgebra de Lie, e com decomposição de Cartan respeito $T$ dada por: $L=T \oplus \sum_{\xi \in G} \oplus L_{\xi}$, onde $G:=\langle\alpha, \beta, \gamma\rangle$ é um grupo abeliano elementar de ordem 8 , e $\operatorname{dim}_{k}\left(L_{\xi}\right)=2$ para todo $\xi \in G$.

Agora vejamos que acontece se $(L,[2])$ contém uma 2-subálgebra de Lie, não simples e não solúvel. 


\subsection{2 (L, $[2])$ contém 2-subálgebras de Lie não simples e não solúveis.}

Afirmação. (L, [2]) contém uma subálgebra de Lie simples de dimensão 3.

De fato, sejam $e_{\alpha} \in L_{\alpha}$ e $e_{\beta} \in L_{\beta}$ elementos da base de $L_{\alpha}$ e $L_{\beta}$ respectivamente, e seja

$$
e_{\alpha+\beta}:=\left[e_{\alpha}, e_{\beta}\right] \in L_{\alpha+\beta} .
$$

Além disso,

$$
e_{\alpha}^{[2]}=\delta t_{2}+\eta t_{3} \quad e e_{\beta}^{[2]}=\lambda t_{1}+\xi t_{3}
$$

$\operatorname{com} \delta, \eta, \lambda, \xi \in \mathbb{F}_{2}$. Logo

$$
\begin{aligned}
& {\left[e_{\alpha}, e_{\alpha+\beta}\right]=\left[e_{\alpha},\left[e_{\alpha}, e_{\beta}\right]\right]=\left[e_{\alpha}^{[2]}, e_{\beta}\right]=\delta e_{\beta} .} \\
& {\left[e_{\beta}, e_{\alpha+\beta}\right]=\left[e_{\beta},\left[e_{\alpha}, e_{\beta}\right]\right]=\left[e_{\beta}^{[2]}, e_{\alpha}\right]=\lambda e_{\alpha} .}
\end{aligned}
$$

Assim, $M_{\alpha, \beta}:=\operatorname{span}\left\{e_{\alpha}, e_{\beta}, e_{\alpha+\beta}\right\}$ é uma subálgebra de Lie de $L$ com tabela de multiplicação de Lie dada por:

\begin{tabular}{|c||c|c|c|}
\hline & $e_{\alpha}$ & $e_{\beta}$ & $e_{\alpha+\beta}$ \\
\hline \hline$e_{\alpha}$ & 0 & $e_{\alpha+\beta}$ & $\delta e_{\beta}$ \\
\hline$e_{\beta}$ & $e_{\alpha+\beta}$ & 0 & $\lambda e_{\alpha}$ \\
\hline$e_{\alpha+\beta}$ & $\delta e_{\beta}$ & $\lambda e_{\alpha}$ & 0 \\
\hline
\end{tabular}

Tabela 4.11: A subálgebra $M_{\alpha, \beta}$

$\operatorname{com} \delta, \lambda \in \mathbb{F}_{2}$.

- Se $\delta=\lambda=0$, então $M_{\alpha, \beta}$ é uma subálgebra de Lie nilpotente de $L$.

- Se $(\delta=0$ e $\xi=1)$ ou $(\delta=1$ e $\xi=0)$, então $M_{\alpha, \beta}$ é uma subálgebra solúvel de $L$.

- Se $\delta=1$ e $\lambda=1$, então $M_{\alpha, \beta}$ é uma subálgebra simples de $L$ e $M_{\alpha, \beta} \simeq \mathfrak{o}_{3}(k)$.

Portanto, $L$ contém uma subálgebra de Lie simples de dimensão 3 isomorfa a $\mathfrak{o}_{3}(k)$. Seja $V$ um $k$-espaço vetorial com base $v_{1}, v_{2}, v_{3}$, e seja $\rho: \mathfrak{o}_{3}(k) \rightarrow \mathfrak{g l}(V)$ uma representação de $\mathfrak{o}_{3}(k)$ em $V$ dada pela tabela seguinte:

\begin{tabular}{|l||c|c|c|}
\hline & $e_{1}$ & $e_{2}$ & $e_{3}$ \\
\hline \hline$v_{1}$ & 0 & $v_{3}$ & $v_{2}$ \\
\hline$v_{2}$ & $v_{3}$ & 0 & $v_{1}$ \\
\hline$v_{3}$ & $v_{2}$ & $v_{1}$ & 0 \\
\hline
\end{tabular}

Tabela 4.12: $O \mathfrak{o}_{3}(k)$-módulo adjunto $V$

Essa representação é irredutível, isomorfa a representação adjunta de $\mathfrak{o}_{3}(k)$. Além disso, em [Do] se demonstra que ela é a única (a menos de isomorfismo) representação irredutível de $\mathfrak{o}_{3}(k)$ de dimensão 3 .

Agora, consideremos as seguintes extensões dos $\mathfrak{o}_{3}(k)_{2}$-2-módulos (ver exemplo 1.5): 
4.1. $L=T \oplus \sum_{\xi \in G} \oplus L_{\xi}, O N D E D I M_{K}\left(L_{\xi}\right)=2, \forall \xi \in G$.

- $k t_{3} \wedge V$.

- $V \wedge k t_{3}$.

dadas nas seguintes tabelas: para $\lambda, \mu, \omega$ e, $\theta$ em $k$ temos:

\begin{tabular}{|c||c|c|c|c|c|}
\hline & $h_{1}$ & $h_{2}$ & $e_{1}$ & $e_{2}$ & $e_{3}$ \\
\hline \hline$v_{1}$ & 0 & $v_{1}$ & 0 & $v_{3}$ & $v_{2}$ \\
\hline$v_{2}$ & $v_{2}$ & 0 & $v_{3}$ & 0 & $v_{1}$ \\
\hline$v_{3}$ & $v_{3}$ & $v_{3}$ & $v_{2}$ & $v_{1}$ & 0 \\
\hline$t_{3}$ & 0 & 0 & $\lambda v_{1}$ & $\mu v_{2}$ & $(\lambda+\mu) v_{3}$ \\
\hline
\end{tabular}

Tabela 4.13: $O \mathfrak{o}_{3}(k)_{2}-2$-módulo $k t_{3} \wedge V$.

\begin{tabular}{|c||c|c|c|c|c|}
\hline & $h_{1}$ & $h_{2}$ & $e_{1}$ & $e_{2}$ & $e_{3}$ \\
\hline \hline$v_{1}$ & 0 & $v_{1}$ & $\omega t_{3}$ & $v_{3}$ & $v_{2}$ \\
\hline$v_{2}$ & $v_{2}$ & 0 & $v_{3}$ & $\theta t_{3}$ & $v_{1}$ \\
\hline$v_{3}$ & $v_{3}$ & $v_{3}$ & $v_{2}$ & $v_{1}$ & $(\omega+\theta) t_{3}$ \\
\hline$t_{3}$ & 0 & 0 & 0 & 0 & 0 \\
\hline
\end{tabular}

Tabela 4.14: $O \mathfrak{o}_{3}(k)_{2}-2$-módulo $V \wedge k t_{3}$.

$\mathrm{O}_{\mathfrak{o}_{3}}(k)_{2}$-2-módulo $k t_{3} \wedge V$ depende dos parametros $\lambda$ e $\mu$, isto é, $k t_{3}<V:=E(\lambda, \mu) \operatorname{com} \lambda, \mu \in k$. Além do mais,

$$
E(\lambda, \mu) \simeq E\left(\lambda^{\prime}, \mu^{\prime}\right) \Longleftrightarrow \lambda=\lambda^{\prime}, \mu=\mu^{\prime} \text { e } k t_{3} \curlywedge V \simeq V \oplus k t_{3} \Longleftrightarrow \lambda=\mu=0 .
$$

O mesmo acontece com o $\mathfrak{o}_{3}(k)_{2}$-2-módulo $V \wedge k t_{3}$.

Assim, tem-se os $k$-espaços vetoriais:

- $S_{1}:=\mathfrak{o}_{3}(k)_{2} \oplus\left(k t_{3} \wedge V\right)$.

- $S_{2}:=\mathfrak{o}_{3}(k)_{2} \oplus\left(V<k t_{3}\right)$.

$S_{1}$ tem as seguintes propriedades:

- $\left(S_{1},[2]\right)$ é uma 2-subálgebra de Lie de $(L,[2])$ de dimensão 9. De fato, $S_{1}$ é uma álgebra de Lie com colchete definido por:

$$
[a+x, b+y]:=(x . b-y . a)+[x, y], \forall a, b \in k t_{3} \wedge V, x, y \in \mathfrak{o}_{3}(k)_{2},
$$

e a 2-aplicação é dada por:

$$
(a+x)^{[2]}:=x . a+x^{[2]}, \forall x \in \mathfrak{o}_{3}(k)_{2}, a \in k t_{3} \wedge V .
$$

- $k t_{3} \wedge V$ é um ideal de $S_{1}$ (portanto, $k t_{3} \wedge V$ é uma subálgebra de $\left.S_{1}\right)$ e $\mathfrak{o}_{3}(k)_{2}$ é uma subálgebra de $S_{1}$. 
- $S_{1}$ não é solúvel. De fato, se $S_{1}$ for solúvel, então toda subálgebra de $S_{1}$ é solúvel, $\operatorname{logo} \mathfrak{o}_{3}(k)_{2}$ é uma subálgebra solúvel de $S_{1}$, o que é uma contradição.

- $S_{1}$ é única, a menos das constantes $\lambda$ e $\mu$.

O mesmo acontece com $S_{2}$.

Assim, $S_{1}$ e $S_{2}$ são 2-álgebras de Lie não simples e não solúveis de dimensão 9.

Em resumo, temos que $(L,[2])$ só contém duas 2 -subálgebras de Lie $S_{i}, i=1,2$, não simples e não solúveis de dimensão 9 . Semelhante a seção 4.1, tomando agora representações de $\left(S_{i},[2]\right)$ ao invés de $\left(\mathfrak{s l}_{3}(k), 2\right)$, vai ser apresentada aqui uma realização de $(L,[2])$ baseada na representação de $S_{i}$ em $k$-espaços vetoriaes $E$, de dimensão 8 . A álgebra $(L,[2])$ vai ser construída no espaço vetorial

$$
G(E):=S_{i} \oplus E .
$$

onde $S_{i}$ é definida como acima e $E$ é um $S_{i}$-2-módulo de dimensão 8.

No que segue, vamos encontrar os $S_{i}$-2-módulos $E$ de dimensão 8. Para isso, seja $U:=\left\langle u_{1}, u_{2}\right\rangle$ um $k t_{3} \wedge V$ (ou $V \wedge k t_{3}$ )-2-módulo de dimensão 2, com base $\left\{u_{1}, u_{2}\right\}$ precisamos achar todos os $S_{i}$-2-módulos induzidos por $U$. Primeiro procuremos as 2representações de $k t_{3} \wedge V$ e $V \wedge k t_{3}$ em $U$, como $u_{i} \cdot t_{3}=u_{i}$ para $i=1,2$. e, $V$ é uma subálgebra abeliana de dimensão 3 (já que, $[V, V]=0$ ), então só basta achar as 2-representações $\rho: V \rightarrow \mathfrak{g l}(U)$

a. 2-representações de $V$ em $U$.

Seja $\rho: V \rightarrow \mathfrak{g l}(U)$ uma representação não fiel de $V$ em $U$ (Já que se $\rho: V \rightarrow \mathfrak{g l}(U)$ é uma representação fiel de $V$ em $U$, então $V \simeq \operatorname{Im}(\rho)$ assim, $V$ é uma subálgebra abeliana de dimensão 3 de $\mathfrak{g l}(U) \simeq \mathfrak{g l}_{2}(k)$, mas $\mathfrak{s l}_{2}(k)$ é a única $k$-álgebra de Lie solúvel de dimensão 3 de $\mathfrak{g l}_{2}(k)$, portanto $V \simeq \mathfrak{s l}_{2}(k)$, o qual é uma contradição, já que $\mathfrak{s l}_{2}(k)$ não é abeliana, pois $\mathfrak{s l}_{2}(k)^{(1)}=\operatorname{span}\left\{e_{11}+e_{22}\right\} \neq\{0\}$.)

Como $\rho$ é um homomorfismo de álgebra de Lie então, para todo $x, y \in V$ temos:

$$
0=\rho(0)=\rho([x, y])=[\rho(x), \rho(y)]=\rho(x) \rho(y)-\rho(y) \rho(x) .
$$

Assim que, $\rho(x) \rho(y)=\rho(y) \rho(x)$ para todo $x, y \in V$. Além disso, como $\rho(x)^{2}=\rho\left(x^{[2]}\right)$ para todo $x \in V$ então $\rho(x)^{2}=0$ para todo $x \in V$. Também, como $\rho: V \rightarrow \mathfrak{g l}(U) \simeq \mathfrak{g l}_{2}(k)$ é não fiel, então existe $x \neq 0$ em $V$ tal que $\rho(x)=0$, seja $x:=v_{1}$ assim $\rho\left(v_{1}\right)=0_{2 \times 2}$. Agora, $\rho\left(v_{i}\right): U \rightarrow U, i=2,3$ são transformações lineares que tem associada uma matriz $A$ de ordem $2 \times 2$ em alguma base de $U$. Então $A$ é semelhante sobre $k$ exatamente a uma matriz dos tipos:

$$
\begin{aligned}
A_{c} & :=\left(\begin{array}{ll}
c & 0 \\
0 & c
\end{array}\right) \\
A_{a, b} & :=\left(\begin{array}{ll}
0 & a \\
1 & b
\end{array}\right)
\end{aligned}
$$


Para todo $a, b, c \in k$.

Se $\rho\left(v_{2}\right)=A_{c}$, e como $0=\rho\left(v_{2}\right)^{2} \Longleftrightarrow c=0$. Então $\rho\left(v_{2}\right)=0_{2 \times 2}$. Logo $\rho\left(v_{3}\right)=A_{a, b}$. Até aqui temos que $\rho\left(v_{i}\right) \rho\left(v_{j}\right)=\rho\left(v_{j}\right) \rho\left(v_{i}\right)$. Além disso, $\rho\left(v_{3}\right)^{2}=0 \Longleftrightarrow A_{a b}^{2}=0_{2 \times 2} \Longleftrightarrow a=b=0$. Nesse caso, $\rho\left(v_{3}\right):=e_{21}$.

Assim, uma possibilidade para definir $\rho: V \rightarrow \mathfrak{g l}(U)$ é:

$$
\begin{aligned}
& v_{1} \mapsto 0_{2 \times 2} \\
& v_{2} \mapsto 0_{2 \times 2} \\
& v_{3} \mapsto A_{0,0}=e_{21}
\end{aligned}
$$

Outra tentativa para definir $\rho: V \rightarrow \mathfrak{g l}(U)$ é:

$$
\begin{aligned}
& v_{1} \mapsto 0_{2 \times 2} \\
& v_{2} \mapsto e_{21} \\
& v_{3} \mapsto B_{2 \times 2}
\end{aligned}
$$

onde $B$ é qualquer matriz $2 \times 2$ sobre $k$, tal que $e_{21} B=B e_{21}$ e $B^{2}=0_{2 \times 2}$. De fato, seja

$$
B:=\left(\begin{array}{cc}
\alpha & \beta \\
\eta & \delta
\end{array}\right)
$$

Então:

- $e_{21} B=B e_{21} \Longleftrightarrow \alpha=\delta$ e $\beta=0$.

- $B^{2}=0_{2 \times 2} \Longleftrightarrow \alpha^{2}+\beta \eta=\eta(\alpha+\delta)=\beta(\alpha+\delta)=\delta^{2}+\eta \beta=0$. daí se conclui que,

$$
\begin{gathered}
\eta \neq 0 \Longrightarrow \alpha=\delta . \\
\eta=0 \Longrightarrow \alpha=\delta=0 .
\end{gathered}
$$

portanto, $B=\alpha\left(e_{11}+e_{22}\right)+\eta e_{21} \forall \alpha, \eta \in k, \eta \neq 0$, ou $B=0_{2 \times 2}$ e assim, $\rho: V \rightarrow \mathfrak{g l}(U)$ é dada por:

$$
\begin{aligned}
v_{1} & \mapsto 0_{2 \times 2} \\
v_{2} & \mapsto e_{21} \\
v_{3} & \mapsto \alpha\left(e_{11}+e_{22}\right)+\eta e_{21}
\end{aligned}
$$

$\mathrm{ou}$

Em resumo, as 2-representações de $V$ em $U$ é dada por:

$$
\begin{array}{lll}
V & \stackrel{\rho_{\alpha, \eta}}{\longrightarrow} & \mathfrak{g l}(U) \\
v_{1} & \mapsto & 0_{2 \times 2} \\
v_{2} & \mapsto & e_{21} \\
v_{3} & \mapsto & \alpha\left(e_{11}+e_{22}\right)+\eta e_{21}
\end{array}
$$

Para todo $\alpha, \eta \in k$.

Além disso, quando $\alpha=0$ e $\eta=0$ temos que $\rho_{0,0}$ é isomorfa a: 


$$
\begin{array}{lll}
v_{1} & \mapsto 0_{2 \times 2} \\
v_{2} & \mapsto 0_{2 \times 2} \\
v_{3} & \mapsto e_{21}
\end{array}
$$

Logo, temos o seguinte $V$-2-módulo $U$ :

\begin{tabular}{|c||c|c|c|}
\hline & $v_{1}$ & $v_{2}$ & $v_{3}$ \\
\hline \hline$u_{1}$ & 0 & $u_{2}$ & $\alpha u_{1}+\eta u_{2}$ \\
\hline$u_{2}$ & 0 & 0 & $\alpha u_{2}$ \\
\hline
\end{tabular}

Tabela 4.15: $O V$-2-módulo $U$.

para todo $\alpha, \eta \in k$

O $V$-2-módulo $U$ depende de dois parametros $\alpha$ e $\eta$, isto é, $U:=E(\alpha, \eta)$ e, assim

$$
E(\alpha, \eta) \simeq E\left(\alpha^{\prime}, \eta^{\prime}\right) \Longleftrightarrow \alpha=\alpha^{\prime} \text { e } \eta=\eta^{\prime} .
$$

b. $S_{1}$-2-módulos induzidos por $U$.

Tem-se que: $k t_{3} \wedge V$ é uma 2-subálgebra de Lie de $S_{1}, U:=\left\langle u_{1}, u_{2}\right\rangle$ o $k t_{3} \wedge V$-2módulo, e $\left\{h_{1}, h_{2}, e_{1}, e_{2}, e_{3}\right\}$ é a base que complementa a $k t_{3} \wedge V$ em $S_{1}$.

Então $\Pi_{1}:=\operatorname{Ind}\left(U, S_{1}\right)$, o $S_{1}$-2-módulo induzido por $U$, tem como base ao conjunto:

$$
\left\{u_{i}, u_{i} e_{1}, u_{i} e_{2}, u_{i} e_{3},\left(u_{i} e_{1}\right) e_{2},\left(u_{i} e_{1}\right) e_{3},\left(u_{i} e_{2}\right) e_{3},\left(\left(u_{i} e_{1}\right) e_{2}\right) e_{3}: u_{i} \in U, i=1,2\right\} .
$$

$\Pi_{1}$ é um $S_{1}$-2-módulo de dimensão 16 cuja tabela de $k t_{3} \wedge V$-ação é dada na página seguinte (ver tabela 4.16).

Para simplificar a notação fazemos $p_{1}:=\left(u_{1} e_{1}\right) e_{2}, p_{2}:=\left(u_{1} e_{1}\right) e_{3}, p_{3}:=\left(u_{1} e_{2}\right) e_{3}$, $q_{1}:=\left(u_{2} e_{1}\right) e_{2}, q_{2}:=\left(u_{2} e_{1}\right) e_{3}, q_{3}:=\left(u_{2} e_{2}\right) e_{3}, p:=\left(\left(u_{1} e_{1}\right) e_{2}\right) e_{3}, q:=\left(\left(u_{2} e_{1}\right) e_{2}\right) e_{3}$, $g_{i}:=u_{1} e_{1}$, e $f_{i}:=u_{2} e_{i}$, para todo $i=1,2,3$. 


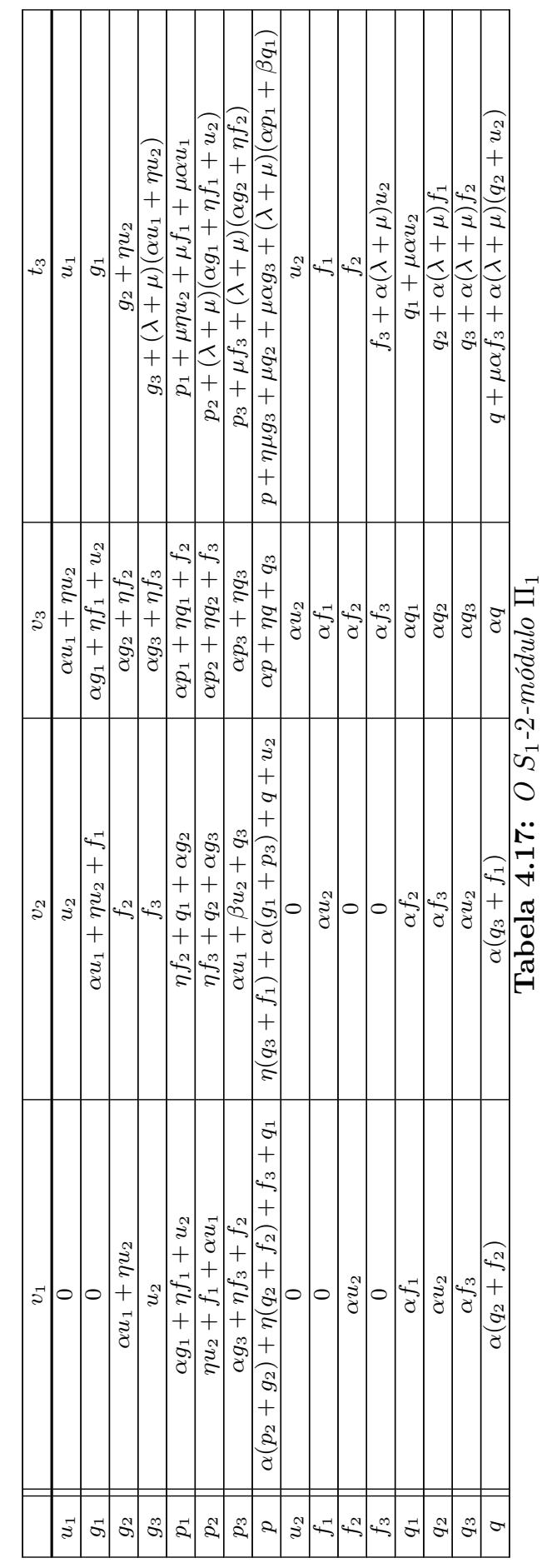


Da tabela anterior vemos que:

$$
E_{S_{1}}:=\left\langle\left\{u_{2}, u_{2} e_{1}, u_{2} e_{2}, u_{2} e_{3},\left(u_{2} e_{1}\right) e_{2},\left(u_{2} e_{1}\right) e_{3},\left(u_{2} e_{2}\right) e_{3},\left(\left(u_{2} e_{1}\right) e_{2}\right) e_{3}\right\}\right\rangle
$$

é um $S_{1}$-2-submódulo de $\Pi_{1}$ de dimensão 8 . Além disso,

$\Pi_{1} / E_{S_{1}}$ também é um $S_{1}$-2-módulo de dimensão 8 , com tabela dada por:

\begin{tabular}{|c||c|c|c|c|}
\hline & $v_{1}$ & $v_{2}$ & $v_{3}$ & $t_{3}$ \\
\hline$u_{1}+E_{S_{1}}$ & $E_{S_{1}}$ & $E_{S_{1}}$ & $\alpha u_{1}+E_{S_{1}}$ & $u_{1}+E_{S_{1}}$ \\
\hline$g_{1}+E_{S_{1}}$ & $E_{S_{1}}$ & $\alpha u_{1}+E_{S_{1}}$ & $\alpha g_{1}+E_{S_{1}}$ & $g_{1}+E_{S_{1}}$ \\
\hline$g_{2}+E_{S_{1}}$ & $\alpha u_{1}+E_{S_{1}}$ & $E_{S_{1}}$ & $\alpha g_{2}+E_{S_{1}}$ & $g_{2}+E_{S_{1}}$ \\
\hline$g_{3}+E_{S_{1}}$ & $E_{S_{1}}$ & $E_{S_{1}}$ & $\alpha g_{3}+E_{S_{1}}$ & $g_{3}+\alpha(\lambda+\mu) u_{1}+E_{S_{1}}$ \\
\hline$p_{1}+E_{S_{1}}$ & $\alpha g_{1}+E_{S_{1}}$ & $\alpha g_{2}+E_{S_{1}}$ & $\alpha p_{1}+E_{S_{1}}$ & $p_{1}+\mu \alpha u_{1}+E_{S_{1}}$ \\
\hline$p_{2}+E_{S_{1}}$ & $\alpha u_{1}+E_{S_{1}}$ & $\alpha g_{3}+E_{S_{1}}$ & $\alpha p_{2}+E_{S_{1}}$ & $p_{2}+\alpha(\lambda+\mu) g_{1}+E_{S_{1}}$ \\
\hline$p_{3}+E_{S_{1}}$ & $\alpha g_{3}+E_{S_{1}}$ & $\alpha u_{1}+E_{S_{1}}$ & $\alpha p_{3}+E_{S_{1}}$ & $p_{3}+\alpha(\lambda+\mu) g_{2}+E_{S_{1}}$ \\
\hline$p+E_{S_{1}}$ & $\alpha\left(p_{2}+g_{2}\right)+E_{S_{1}}$ & $\alpha\left(g_{1}+p_{3}\right)+E_{S_{1}}$ & $\alpha p+E_{S_{1}}$ & $p+\mu \alpha g_{3}+\alpha(\lambda+\mu) p_{1}+E_{S_{1}}$ \\
\hline
\end{tabular}

Tabela 4.19: $O S_{1}-2$-módulo $\Pi_{1} / E_{S_{1}}$

Mas das tabelas 4.16 e 4.18 podemos concluir que $E_{S_{1}} \simeq \Pi_{1} / E_{S_{1}}$ como $S_{1}$-2módulos. E assim só temos um $S_{1}$-2-módulos $E_{S_{1}}$ de dimensão 8 que depende dos parametros $\alpha, \lambda, \mu$.

c. $S_{2}$-2-módulos induzidos por $U$.

Seja $\Pi_{2}:=\operatorname{Ind}\left(U, S_{2}\right)$ o $S_{2}$-2-módulo induzido por $U$, com base:

$$
\left\{u_{i}, u_{i} e_{1}, u_{i} e_{2}, u_{i} e_{3},\left(u_{i} e_{1}\right) e_{2},\left(u_{i} e_{1}\right) e_{3},\left(u_{i} e_{2}\right) e_{3},\left(\left(u_{i} e_{1}\right) e_{2}\right) e_{3}: u_{i} \in U, i=1,2\right\} .
$$

$\Pi_{2}$ é um $S_{2}$-2-módulo de dimensão 16 cuja tabela de $V \wedge k t_{3}$-ação é dada na página seguinte (ver tabela 4.20).

Para simplificar a notação fazemos $p_{1}:=\left(u_{1} e_{1}\right) e_{2}, p_{2}:=\left(u_{1} e_{1}\right) e_{3}, p_{3}:=\left(u_{1} e_{2}\right) e_{3}$, $q_{1}:=\left(u_{2} e_{1}\right) e_{2}, q_{2}:=\left(u_{2} e_{1}\right) e_{3}, q_{3}:=\left(u_{2} e_{2}\right) e_{3}, p:=\left(\left(u_{1} e_{1}\right) e_{2}\right) e_{3}, q:=\left(\left(u_{2} e_{1}\right) e_{2}\right) e_{3}$, $g_{i}:=u_{1} e_{i}$ e, $f_{i}:=u_{2} e_{i}$ para todo $i=1,2,3$. 


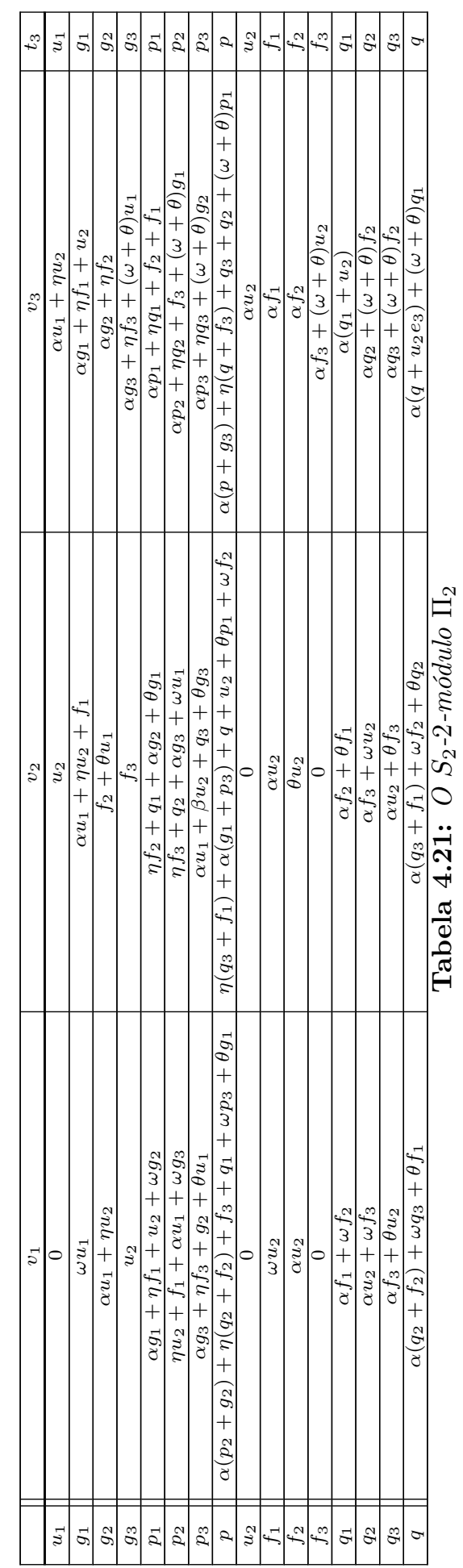


Da tabela anterior vemos que

$$
E_{S_{2}}:=\left\langle\left\{u_{2}, u_{2} e_{1}, u_{2} e_{2}, u_{2} e_{3},\left(u_{2} e_{1}\right) e_{2},\left(u_{2} e_{1}\right) e_{3},\left(u_{2} e_{2}\right) e_{3},\left(\left(u_{2} e_{1}\right) e_{2}\right) e_{3}\right\}\right\rangle
$$

é um $S_{2}$-2-submódulo de $\Pi_{2}$ de dimensão 8. Além disso,

$\Pi_{2} / E_{S_{2}}$ também é um $S_{2}$-2-módulo de dimensão 8 , com tabela:

\begin{tabular}{|c|c|c|c|c|}
\hline & $v_{1}$ & $v_{2}$ & $v_{3}$ & $t_{3}$ \\
\hline$u_{1}+E_{S_{2}}$ & $E_{S_{2}}$ & $E_{S_{2}}$ & $\alpha u_{1}+E_{S_{2}}$ & $u_{1}+E_{S_{2}}$ \\
\hline$g_{1}+E_{S_{2}}$ & $\omega u_{1}+E_{S_{2}}$ & $\alpha u_{1}+E_{S_{2}}$ & $\alpha g_{1}+E_{S_{2}}$ & $g_{1}+E_{S_{2}}$ \\
\hline$g_{2}+E_{S_{2}}$ & $\alpha u_{1}+E_{S_{2}}$ & $\theta u_{1}+E_{S_{2}}$ & $\alpha g_{2}+E_{S_{2}}$ & $g_{2}+E_{S_{2}}$ \\
\hline$g_{3}+E_{S_{2}}$ & $E_{S_{2}}$ & $E_{S_{2}}$ & $\alpha g_{3}+(\omega+\theta) u_{1}+E_{S_{2}}$ & $g_{3}+E_{S_{2}}$ \\
\hline$p_{1}+E_{S_{2}}$ & $\alpha g_{1}+\omega g_{2}+E_{S_{2}}$ & $\alpha g_{2}+\theta g_{1}+E_{S_{2}}$ & $\alpha p_{1}+\alpha u_{1}+E_{S_{2}}$ & $p_{1}+E_{S_{2}}$ \\
\hline$p_{2}+E_{S_{2}}$ & $\alpha u_{1}+\omega g_{3}+E_{S_{2}}$ & $\alpha g_{3}+\omega u_{1}+E_{S_{2}}$ & $\alpha p_{2}+(\omega+\theta) g_{1}+S_{2}$ & $p_{2}$ \\
\hline$p_{3}+E_{S_{2}}$ & $\alpha g_{3}+\theta u_{1}+E_{S_{2}}$ & $\alpha u_{1}+\theta g_{3}+E_{S_{2}}$ & $\alpha p_{3}+(\omega+\theta) g_{2}+E_{S_{2}}$ & $p_{3}$ \\
\hline$p+E_{S_{2}}$ & $\alpha\left(p_{2}+g_{2}\right)+\omega p_{3}+\theta g_{1}+E_{S_{2}}$ & $\alpha\left(g_{1}+p_{3}\right)+\omega g_{2}+\theta p_{2}+E_{S_{2}}$ & $\alpha\left(p+g_{3}\right)+(\theta+\omega) p_{1}+E_{S_{2}}$ & $p+E_{S_{2}}$ \\
\hline
\end{tabular}

Tabela 4.23: $O S_{2}-2$-módulo $\Pi_{2} / E_{S_{2}}$

Como no caso anterior, das tabelas 4.20 e 4.22 temos que $E_{S_{2}} \simeq \Pi_{2} / E_{S_{2}}$ como $S_{2^{-}}$ 2-módulos. E assim só temos um $S_{2}$-2-módulos $E_{S_{2}}$ de dimensão 8 que depende dos parametros $\alpha, \theta, \omega$.

Em resumo, a $k$-álgebra $(L,[2])$ então é construída sobre o $k$-espaço vetorial

$$
G\left(E_{S_{i}}\right):=S_{i} \oplus E_{S_{i}}, \quad \text { para } i=1,2 .
$$

Da mesma maneira como no caso simples (seção 4.1), nós precisamos saber se $G\left(E_{S_{i}}\right)$ é uma $k$-álgebra, isto é, precisamos de condições necessarias e suficientes (como no Teorema 3.2) para dar uma resposta a seguinte pergunta:

Pergunta: Será que existe um $S_{i}$-morfismo $\varphi: \Lambda^{2}\left(E_{S_{i}}\right) \rightarrow S_{i}$ ?

Observação 4.3. Essa questão, e o caso quando $(L,[2])$ contém 2-subálgebras de Lie solúveis, serão objetos de pesquisas futuras. 


\section{Referências Bibliográficas}

[Do] DOLOTKAZIN, A., Irreducible representations of a three-dimensional simple Lie algebras of characteristic $p=2$, Math. Note 24 (1978), $588-590.6$

[Gr] GRICHKOV, A.N., On simple Lie algebras over a field of characteristic 2, J. Algebra 363 (2012), $14-18$.

[GP] GRICHKOV, A.N., PREMET, A.A., Simple Lie algebras of absolute toral rank 2 in characteristic 2 (submitted).

[GGA] GRICHKOV, A.N., ARAUJO, W., As álgebras de Lie simples de dimensão 7 sobre um corpo de característica 2 e suas subálgebras toroidais, IME-USP, 2014.

[GZ] GRICHKOV, A.N., ZUSMANOVICH,P.,Deformations of current Lie algebras. I. Small algebra in characteristic 2, arXiv: 1410.3645

[Ja] JACOBSON, N., Lie Algebra, Interscience, New York,1962.

[KV] KAC, V.,VEǏSFEILLER, B., Exponentials in Lie algebras of characteristic $p$, Math.USSR Izvestija, vol.5 (1971), No. 4

[Sk] SKRYABIN, S., Toral rank one simple Lie algebras of low characteristic, J. Algebra 200 (1998), 650-700.

[SF] STRADE, H., FARNSTEINER, R., Modular Lie Algebra and Their Representations, Marcel Dekker, New York, 1988.

[St] STRADE, H.,Lie algebras of small dimension, htt//arXiv.org/abc/math/0601413. 17 jan 2006.

[St1 ] STRADE. H.,Simple Lie Algebras over Fiels of Positive characteristic, Volume I: Structure Theory, DeGruyter Expositions in Math., Vol. 38, Berlin, 2004.

[St2] STRADE,H.,Simple Lie Algebras over Fiels of Positive characteristic, Volume II: Classifying the Absolute Toral Rank Two case. DeGruyter Expositions in math., vol. 42, Berlin, 2009. 
[St3] STRADE. H.,Simple Lie Algebras over Fiels of Positive characteristic, Volume III: Completion of the Classification, DeGruyter Expositions in Math., Vol. 57, Berlin, 2012. 